\title{
A Guide to Tables of Bessel Functions
}

\author{
by \\ HARRY BATEMAN \\ Raymond Clare Archibald
}

\section{Contents}

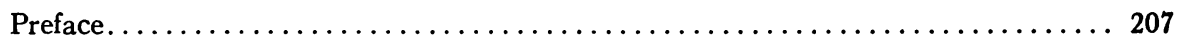

General Remarks on Notation................................ 209

Part I: Guide to Tables and Graphs; Polynomial Approximations and Asymptotic

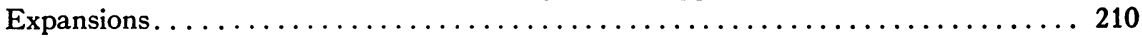

I-Bessel Functions of the First Kind and Integral Order $J_{n}(x) \ldots \ldots \ldots \ldots 210$

Introduction. $\mathbf{A}_{1} . J_{0}(x)$ and $J_{1}(x)$. $A_{2} . J_{0}(x)$ or $J_{1}(x)$; functions involving $J_{0}(x)$ and (or) $J_{1}(x)$. $\mathbf{B}_{1}$. Zeros: $x_{8}$ of $J_{0}(x)$, and $J_{1}\left(x_{8}\right)$. $\mathbf{B}_{2}$. Zeros: $x_{8}$ only. $\mathbf{B}_{8}$. Zeros: functions of $x_{8}$. $\mathbf{B}_{4}$. Zeros: $x_{8}^{\prime}$ of $J_{1}(x)$, and $J_{0}\left(x_{8}{ }^{\prime}\right)$. $\mathbf{B}_{5}$. Zeros: $x_{8}^{\prime}$ only. $\mathbf{B}_{60}$ Zeros: functions of $x_{8}^{\prime} . \mathrm{B}_{7}$. Zeros: functions of $J_{0}(x)$ and $J_{1}(x) . \mathrm{C}_{1} . J_{n}(x)$, including $n>1 . \mathrm{C}_{2}$. Functions, derivatives, and differences of $J_{n}(x)$. $\mathbf{D}_{1}$. Zeros: $j_{n, s}$ including $n>1$. $\mathbf{D}_{2 .}$ Zeros: $j_{2, s / \pi}$, and miscellaneous. E. Graphs, reliefs.

II-Bessel Functions of the Second Kind and Integral Orders $Y_{n}(x), Y^{(n)}(x)$,

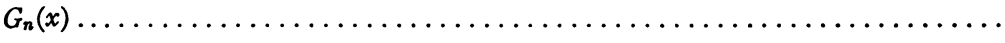

Introduction. $\mathbf{A}_{1}, Y_{0}(x)$ and $Y_{1}(x) . \mathbf{A}_{2} \cdot Y_{n}(x)$, including $n>1$. $\mathbf{A}_{3}$. Functions involving $Y_{n}(x)$ and derivatives. $\mathbf{A}_{4}$. Zeros: $y_{8}$ of $Y_{0}(x), y_{8}^{\prime}$ of $Y_{1}(x)$. A $A_{6}$ Zeros: $y_{n, 8}$ of $Y_{n}(x)$, and of functions of $Y_{n}(x)$. $A_{6}$. Graphs: $Y_{n}(x)$, etc. B. $G_{0}(x)=-\frac{1}{2} \pi Y_{0}(x)$ and $G_{1}(x)=-\frac{1}{2} \pi Y_{1}(x) . \mathbf{B}_{2} \cdot G_{n}(x)=-\frac{1}{2} \pi Y_{n}(x)$, and derivatives. $\mathrm{C}_{1} . Y^{(0)}(x)$ and $Y^{(1)}(x) . \mathrm{C}_{2} . Y^{(n)}(x)$, including $n>1 . \mathrm{C}_{3} . Y^{(n)}(x)$, zeros.

III-Modified Bessel Functions of the First and Second Kind- $I_{n}(x), K_{n}(x)$. Bessel Functions of the Third Kind $-H_{n}^{(1)}(x), H_{n}^{(2)}(x) \ldots \ldots \ldots \ldots \ldots \ldots$

Introduction. $\mathrm{A}_{1} . I_{0}(x)$ and $I_{1}(x) . \mathrm{A}_{2} \cdot I_{0}(x)$ or $I_{1}(x)$; functions involving $I_{0}(x)$ and (or) $I_{1}(x) . A_{3} . I_{n}(x)$, including $n$ (integer) $>1$. A 4 . Functions of $I_{n}(x)$. $A_{5}$. Kirchhoff's equation, $\sigma \neq 1$. $A_{60}$ Kirchhoff's equation, $\sigma=1$. $A_{7}$. Graphs involving $I_{n}(x) . \mathbf{B}_{1} . K_{0}(x), K_{1}(x) . \mathbf{B}_{2} . K_{0}(x)$ or $K_{1}(x)$; functions involving $K_{0}(x)$ and (or) $K_{1}(x)$; zeros. $\mathbf{B}_{3} . K_{n}(x)$, including $n$ (integer) $>1$; functions of $K_{n}(x)$; graphs. C. $H_{n}^{(1)}(x)=J_{n}(x)+i Y_{n}(x), H_{n}^{(2)}(x)=J_{n}(x)-i Y_{n}(x)$.

IV-Bessel Functions whose Order is $\pm\left(n+\frac{1}{2}\right), n+1$ a Positive Integer.

Introduction. A. $J_{ \pm(n+1)}(x)= \pm(-1)^{n} Y_{\mp(n+1)}(x)$. B. Riccati-Bessel functions $S_{n}(x), C_{n}(x)$. C. Spherical Bessel functions $j_{n}(x)=(\pi / 2 x) J_{n+3}(x)$, etc. D. $J_{n}(x), I_{n}(x), K_{n}(x), n$ half an odd integer; miscellaneous. E. Zeros. F. Graphs. 
V-Bessel Functions of Order $\pm 1 / 3, \pm 2 / 3$

Introduction. $\mathbf{A}_{1} . J_{ \pm 1 / 3}(x), J_{ \pm 2 / 3}(x), Y_{ \pm 1 / 3}(x), Y_{ \pm 2 / 3}(x) . \mathbf{A}_{2} \cdot I_{ \pm 1 / 3}(x), I_{ \pm 2 / 3}(x)$. $\mathbf{B}_{1} . e^{-x} I_{1 / 3}(x), e^{x} K_{1 / 3}(x),\left|H_{1 / 3}^{(1)}(x)\right|$, arg $H_{1 / 3}^{(1)}(x), u_{1 / 3}(x), v_{1 / 3}(x)$. $\mathbf{B}_{2}$. Other functions. $\mathrm{C}_{1}$. Airy's integral $A(m)$. $\mathrm{C}_{2}$. Airy's integrals $A i(x), B i(x) . \mathrm{C}_{3}$. Solutions of $S^{\prime \prime}=9 x S$. C 4 . Other related Airy integrals. D. Zeros. E. Graphs.

$\mathrm{VI}-J_{n}(x), n= \pm 1 / 4, \pm 3 / 4, \pm 1 / 6, \pm 5 / 6$, etc

Introduction. $\mathbf{A}_{1}, J_{n}(x), Y_{n}(x) . \mathbf{A}_{2} \cdot I_{n}(x)$. B. Zeros. C. Graphs.

VII-Bessel-Clifford Functions $x^{m} J_{n}\left(x^{s}\right)$

Introduction. A. Tables and graphs.

VIII- $J_{n}(x)$ and other Functions, as Functions of the Order $n$

Introduction. A. Tables, graphs, and charts.

IX-The Functions of Lommel, Schläfli, Lommel-Weber, Rayleigh, and Struve. . 244

Introduction. $\mathbf{A}_{1} \cdot \Omega_{0}(x)=H_{0}(x), \Omega_{1}(x), H_{1}(x) . \mathbf{A}_{2} \cdot \Omega_{n}(x)$ and its functions. $\mathbf{B}$.

Miscellaneous. C. Graphs.

$\mathbf{X}$-Integrals involving Bessel Functions

Introduction. A . Integrals involving $J_{n}(x), Y_{n}(x)$. A. Fresnel integrals $S(u)$ and $C(u)$. A. Fresnel integrals $S\left[(2 x / \pi)^{\xi}\right], C\left[(2 x / \pi)^{\natural}\right]$. B. Integrals involving $I_{0}(x), K_{0}(x)$. C. Graphs.

XI-Bessel Functions of a Complex Argument.

Introduction. A. $J_{0}(z), J_{1}(z), Y_{0}(z), Y_{1}(z)$. B $\mathbf{B}_{1}$ ber $x$ and bei $x$. B $\mathbf{B}_{2}$. ber' $x$ and

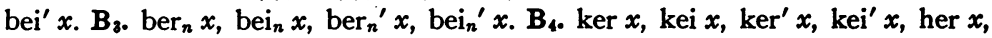
hei $x$, $\operatorname{her}^{\prime} x, \operatorname{hei}^{\prime} x . \mathbf{B}_{5} . \operatorname{ker}_{n} x, \operatorname{kei}_{n} x, \operatorname{ker}_{n}^{\prime} x, \operatorname{kei}_{n}{ }^{\prime} x, \operatorname{her}_{n} x, \operatorname{hei}_{n} x$. C. Michell and Airey functions. $\mathbf{D}_{1} . X, V, W, Z, X^{(k)}, V^{(k)}, W^{(k)}, Z^{(k)}, V^{(r)}, V^{(u)} . \mathbf{D}_{2}$. $\frac{V}{X}, \frac{W}{X}, \frac{Z}{X}, \frac{Z}{V}, \frac{W}{V}$, etc. E. $M_{n}(x), \theta_{n}(x), N_{n}(x), \phi_{n}(x)$. F. Miscellaneous. G. Zeros. H. Graphs and reliefs.

XII-Series of Bessel Functions; Lommel Functions $U_{n}(y, z), V_{n}(y, z)$

Introduction. $\mathbf{A}_{1}$. Lommel functions of a single variable. A2. Lommel functions of two variables. B. Series. C. Graphs.

XIII-Polynomial Approximations and Asymptotic Expansions

A. Introduction. B. Asymptotic series for $J_{n}(x)$ and $Y_{n}(x)$. C. Asymptotic series for $I_{n}(x)$ and $K_{n}(x)$. D. Approximations by means of series of various types. E. Asymptotic expansions of Bessel functions of nearly equal order and argument. F. Series for the zeros of Bessel functions.

Index of Authors referred to in Introductions I-XII and in XIII

Part II: Bibliography of Authors of Tables and Graphs. 


\section{Preface}

Since there is hardly a single field of applied mathematics in which Bessel Functions do not appear, our Committee learned from many sources that a modern Guide to Tables of Bessel Functions would fill a great need at the present time. Only two previous guides are worthy of mention, namely: those of G. N. WATson, A Treatise on the Theory of Bessel Functions, Cambridge, 1922, p. 654-664; and of R. v. Mises, Verzeichnis berechneter Funktionentafeln ... Erster Teil, Besselsche, Kugel- und elliptische Funktionen, Berlin, 1928, p. 7-19, 29-30. After more than a year of preparation to meet this need, Special Number $M T A C 7$ is now being distributed. H. B. had devoted several years of research to his Report on Higher Functions, which was in an advanced stage of preparation for presentation to the Committee, when the call came for this new Guide.

The manuscript of such a Guide in 18 Sections and a Bibliography, selected by H. B. from material of his Report, and embracing most of the matter now presented, was sent to the editors in July 1943. In constant consultation with H. B., R. C. A. worked through this manuscript, considerably modifying its form, and adding new references. In the rearrangement the number of Sections was reduced to 13. In the first manuscript various portions of the present Section XIII, on "Polynomial Approximations and Asymptotic Expansions," were to be found in several Sections. Then came the final stage of constant collaboration of H. B. and R. C. A., checking and rechecking of Sections I-XII. H. B. is alone responsible for XIII.

The general plan of the Guide is indicated by the table of "Contents." There are two main Parts. Part I, divided into 13 Sections, consists chiefly of that portion of the Guide where various known tables and graphs are listed with their authors. Part II is the Bibliography with details concerning the publications of the authors mentioned in Part I, notes on errata, and other bibliographic material of interest. Each of the Sections I-XII of Part I has an Introduction in which not only are notations of the Guide defined but also some indications are given of other notations employed in standard sources. (For "General Remarks on Notation" see at the end of this Preface). Sometimes an Introduction also includes certain formulae and explanatory comment which may be of interest or of assistance to a consulter of the Section. Following the Introductions are references to Tables and Graphs, those of a kind being brought together when possible. For the purposes of this Guide the term Table has been interpreted very freely, since only two or three, or even a single numerical result by a given author may sometimes be listed, because of possible practical service. Since graphical representations are coming into prominence in current papers, memoirs, and books, apology for this first comprehensive assembly of references to such material is hardly necessary.

For each Table in the main part of the Guide the author, and exact range are given. The author is referred to by his name without initials, if there is no other author of this name in the Bibliography, where details of the source are set forth. If there is not more than one entry to the author's credit no number follows his name in a reference. In such a reference as Airey 27, however, this refers to no. 27 of the 35 entries in the Bibliography under the name of AIREy, who easily "leads all the rest" in the total amount of his tabular contributions. When a Table has appeared in more than one edition (in part or as a whole) subscripts are of ten used in connection with the corresponding numbering of entries, as in the case of BOURGET $1_{1}$ to $1_{12}$.

Two features of the Guide which the undersigned regard as of special importance are (1) the complete listing of every known Unpublished Table of Bessel Functions now available for consultation; ${ }^{1}$ (2) the assembly of information regarding known Errors in the tables recorded. In every case we have attempted to indicate the author or the discoverer of the errors, with exact references to their places of publication, when published. In some cases such lists are reprinted. But a number of errata lists appear in print for the first time in this Guide, see, for example, under Bessel, Karas, Maclean, TölkE. Since the listing of such Errata ought materially to assist anyone using material listed in this Guide, we should be grateful for the receipt of reports on other errors so that they may be recorded in later 
numbers of $M T A C$. In a few cases we have put in bold-faced type the name of an author of a table which, for one reason or another, we regarded as of outstanding importance, even though another might be almost as good.

In the Bibliography we have generally given the complete titles of books, doctoral dissertations, and mathematical contributions in programs, to which we have referred, and in cases where a volume contains a number of tables necessary for listing in our Guide (such as JAHNKE \& EMDE, and WATSON 3), we have usually indicated enough of the contents to make clear the type of every such table in each volume. In the case of tables in serials we have however with regret omitted all titles of articles or memoirs to which references are given for tables or graphs. This was done to conserve space, while we fully realized that a suggestion as to the field in which a table was prepared may of itself be of considerable interest. On the other hand the title of the periodical will of ten suggest a partial answer to such a thought, and after all, the chief purpose of this Guide is to answer the questions, Have any tables of such and such functions been published? If so, where and with what ranges?

In the case of various entries of the Bibliography we have intended to give only the page numbers where the tables or graphs may be found. In this way it is in general unnecessary to add page numbers to the author names in the main body of the Guide. In every case, however, where difficulty or delay might arise, page numbers have been added. Two types of such difficulties may be illustrated. There are, e.g., various page-references to the volume by MORSE; the first four references at least are of the form MORSE, p. 175. Again, in listing tables of $Y_{0}(x), Y_{1}(x)$, page references would be given, e.g., to JAHNKE \& EMDE $1_{5}$, p. 190-197, because these functions are there called $N_{0}(x), N_{1}(x)$. Functions in such a source with the same name as in our Guide could be readily found so that our addition of page references to a name would then be unnecessary.

The forms of our titles of serials were determined by the monumental new Union List of Serials (New York, 1943, 3065 large 4to pages) which gives full details concerning ranges of each serial, and tells of the location of all periodicals in many libraries of the United States and Canada. By means of interlibrary loans, and visiting of other libraries we were enabled to inspect practically every listed serial and every listed book or pamphlet. Exceptions are indicated by the usual notation of $M T A C$, with a o before the titles. In the case of some items which we had much difficulty in finding, the names of the libraries owning them have been noted in the Bibliography. Most printed items are, in some form, in the Library of Brown University. Comparatively few of the various mss. which we list have been seen by us, but in no case is a o used here.

We have mentioned that for every author of a table referred to in our Guide there is at least one corresponding entry in the Bibliography. For every main author's name in the Bibliography are Roman numerals such as [I, V, XI] showing the Sections where there are direct or indirect references to tables of the author in question. An example of an indirect reference is XI under HAYASHI 2. This is referred to in the Guide from Schleicher, where the HAyashi edition of the table is listed as $1_{2}$. In Part II are many authors indirectly referred to in Part I. In two cases of Section IX, for example, where tables are listed only in the Introduction, after each of the references in the Bibliography is [IXi].

Names in Introductions, and in Section XIII, are listed in a separate index.

For the most part the nature of our abbreviations will be perfectly obvious. B.A.A.S. $=$ British Association for the Advancement of Science, and BAASMTC = B.A.A.S., Mathematical Tables Committee. So also NYMTP = Mathematical Tables Project, New York; A.I.E.E. = American Institute of Electrical Engineers; A.S.M.E. = American Society of Mechanical Engineers; A.S.C.E. = American Society of Civil Engineers; R.A.S. = Royal Astronomical Society; $\Delta$ is used for first differences, $\Delta^{2}$ for first and second differences, etc., $\delta^{2}$ for second differences only, $\delta_{m}{ }^{2}$ for modified second differences; $6 \mathrm{D}=6$ places of decimals; $6 \mathrm{~S}=6$ significant figures; p. $234 \mathrm{f}=$ p. 234 and one or more of the following pages; $(2 n+1) ! !=1.3 .5 \cdots(2 n+1) ;$ T. = Table; var. = various. In $M T A C$, p. 2 , we have already indicated the basis for our transliterations from Russian. We do not necessarily accept the forms of transliterations of names into English by Russians. 
We are deeply indebted to Professor W. G. BickLey, of the Imperial College of Science and Technology, London, and to Dr. J. C. P. MrLler, for exact information concerning tabular achievements of the remarkable BAASMTC (which has for 55 years been publishing tables of Bessel Functions), and to Dr. MILler for other information, connected with his name in several places.

But especially do we wish to record our great obligations to the Liverpool Index, not only for ten out of a variety of titles which we had overlooked, but also for many suggestions which improved the form of presentation of our material. The ten titles were by the following authors: Buxton, Conrady, Eddington, Hartree \& Johnston, NanCarrow, Nicholson, Silberstein, Smart, Witkowski, Yost Wheeler \& Breit. There is renewed acknowledgment where these names occur in Part II.

In spite of the great care we have taken to be exact and accurate in our statements, numbering many thousands, we can only reasonably hope that our slips of a major nature have not been too numerous. That we have not discovered a number of tables of importance seems almost certain. Reports which we may receive concerning such slips or lacunae will be gratefully acknowledged.

H. B.

R. C. A.

\section{General Remarks on Notation}

The notation used in this Guide has been chosen so as to agree as far as possible with that used by English writers. Thus $J_{n}(x)$ is used for the Bessel function of the first kind and $Y_{n}(x)$ for the Bessel function of the second kind which for large positive values of $x$ have the asymptotic forms

$$
J_{n} \approx\left(\frac{1}{2} \pi x\right)^{-1} \cos X_{n}, \quad Y_{n} \approx\left(\frac{1}{2} \pi x\right)^{-1} \sin X_{n},
$$

respectively, where $X_{n}=x-\left(n+\frac{1}{2}\right) \frac{1}{2} \pi$. The notation $N_{n}(x)$ used for the second function by some German and American writers is not used here partly because the symbol $N_{n}(x)$ is needed for another purpose in Section XI. For real values of $x$ it is convenient to adopt Hankel's notation

$$
H_{n}^{(1)}(x)=J_{n}(x)+i Y_{n}(x), \quad H_{n}^{(2)}(x)=J_{n}(x)-i Y_{n}(x),
$$

for the functions with asymptotic forms

$$
\left(\frac{1}{2} i \pi x\right)^{-\frac{1}{3}} e^{i(x-i n \pi)}, \quad\left(-\frac{1}{2} i \pi x\right)^{-i} e^{-i\left(x-\frac{i n}{2} \pi\right)},
$$

which are so useful in the theory of the propagation of waves. This notation must not be confused with the notation $H_{n}(x)$ used for the Struve function of Section IX, the symbol $S_{n}(x)$ used by JAHNKE \& EMDE being rejected because this symbol is needed in Section IV for one of the Riccati-Bessel functions. As, moreover, the symbol $C_{n}(x)$ is also needed for a Riccati-Bessel function the notation for the Clifford function has been modified into $C l_{n}(x)$. For the zeros of $J_{n}(x)$ the symbol $j_{n, s}$ is used for the $s$-th positive zero but when $n=0$ a special notation $x_{s}$ for the $s$-th positive zero has been adopted, primes being added to denote the zeros of the successive derivatives of this function.

In the asymptotic expansions of $J_{n}(x)$ and $Y_{n}(x)$ the notation used is like that in WHITTAKER \& WATSON, Modern Analysis, except that $U_{n}(x), V_{n}(x)$ have been replaced by $u_{n}(x), v_{n}(x)$.

1 This statement is not now strictly true. After it was written we were privileged to see the first proof of pages (by J. C. P. MILLER) dealing with tables of Bessel functions in the forthcoming Index of Mathematical Tables prepared by the Liverpool mathematicians, A. Fletcher, J. C. P. Miller, and L. Rosenhead. In this Index (which we shall call the "Liverpool Index") are references to more than $30 \mathrm{~ms}$. tables of Bessel Functions, to which we make no other reference. The Scientific Computing Service, London, publisher of the Liverpool Index, hopes that the volume may be simultaneously issued in England and America, late in 1944 or very early in 1945 . Dover Publications is likely to be the American agent. 


\section{PART I}

\section{Guide to Tables and Graphs; Polynomial Approximations and Asymptotic Expansions}

\section{Bessel Functions of the First Kind and Integral ORDER $J_{n}(x)$}

For all values of $n$ a solution of the linear differential equation

$$
x^{2} y^{\prime \prime}+x y^{\prime}+\left(x^{2}-n^{2}\right) y=0
$$

is the function $J_{n}(x)$ defined as

$$
\sum_{s=0}^{\infty}(-1)^{s}\left(\frac{1}{2} x\right)^{n+2 s} /[s !(n+s) !]=\left(\frac{1}{2} x\right)^{n} \Lambda_{n}(x) / n !
$$

a power series which converges for all values of $x$ and so represents an entire function. This solution of the Bessel equation (1) is called a Bessel function of the first kind. ${ }^{1}$ It is obvious that $J_{-n}(x)$ is also a solution, and independent of $J_{n}(x)$ except when $n$ is an integer and $J_{-n}(x)=(-1)^{n} J_{n}(x)$.

The relation (2) may be written

(3) $\Lambda_{n}(x)=n !\left(\frac{1}{2} x\right)^{-n} J_{n}(x)$, and therefore $\Lambda_{0}(x)=J_{0}(x), \Lambda_{1}(x)=2 J_{1}(x) / x$.

For our values of $n, J_{n}(x)$ may also be defined by

$$
\left(\frac{1}{2} x\right)^{n} /\left[\left(n-\frac{1}{2}\right) ! \pi^{\left.\frac{1}{3}\right]} \int_{0}^{\pi} \cos (x \cos \phi) \sin ^{2 n} \phi d \phi .\right.
$$

The series (2) occurred in the solution of Kepler's equation

$$
M=E-e \sin E,
$$

which was found about 1769 by J. L. LAGRANGE when he just used the expansion theorem which he had first discovered to express $E$ as a power series in $e$. He then transformed this series into a trigonometric series

$$
E=M+2 \sum_{n=1}^{\infty} \sin (n M) J_{n}(n e) / n
$$

BESSEL seems to have been the first to use Fourier's rule for the coefficient in a sine series in this particular case. He thus found the expression

$$
J_{n}(x)=(1 / \pi) \int_{0}^{\pi} \cos (n t-x \sin t) d t, \quad n \text { an integer }
$$

when $x$ is real this formula shows that $-1 \leq J_{n}(x) \leq 1$. The function $J_{n}(x)$ was also found to satisfy the recurrence relations

$$
\begin{aligned}
& J_{n-1}(x)+J_{n+1}(x)=(2 n / x) J_{n}(x) \\
& J_{n-1}(x)-J_{n+1}(x)=2 J_{n}^{\prime}(x)
\end{aligned}
$$


which are also satisfied by other suitably defined solutions of the differential equation which are commonly called cylindrical functions.

In investigating the diffraction of an object glass with circular aperture AIRY 2 was led in 1835 to publish a 4-place table of $\Lambda_{1}(x)=2 J_{1}(x) / x$, $x=0(.2) 12$. In 1870 Lommel greatly extended this table. In 1889 MASCART gave tables of $\Lambda_{1}(x)$ and $\Lambda_{1}^{2}(x)$ for the same range but with parameter $m=x / 2=0(.1) 6$. Independently of Airy SchwERD published in 1835, tables of $\Lambda_{1}(x)$ and $\Lambda_{1}^{2}(x)$ for $12 x / \pi=p=[0(1) 75 ; 5 \mathrm{D} p>10,4 \mathrm{D} p \leq 10]$. In degrees the range is $p=0\left(15^{\circ}\right) 1125^{\circ}$, and so Schwerd's table is much more extensive than that of AIRY 2, for which the extreme value of $x$ is 12 , or $p=46\left(690^{\circ}\right)$. Schwerd's tables give 6 maxima and 6 minima values, approximately, 5 outside the range of the larger table, the last values being for $1930^{\circ}$. ( $p$ about 128.7). Thus we have 12 approximate values of zeros for $x J_{1}^{\prime}(x)-J_{1}(x) \equiv x J_{0}(x)-2 J_{1}(x)$.

Already in 1732 Daniel Bernoulli ${ }^{2}$ presented a paper to the Academy at St. Petersburg in which he treated the problem of the vibration of a heavy string suspended from an end, with the solution leading to a Bessel function of the zeroth order; he found approximations (equivalent to 2.406, 5.547 ) to the first two zeros of $J_{0}(x)$. Euler not only arrived at similar results, but discussed all Bessel functions of the first kind of zeroth order. (See Maggi, and Bôcher; ${ }^{2}$ also Section II, footnote 4).

Notation: In what follows the $s$-th positive root of the equation $J_{0}(x)=0$ will be denoted by $x_{8}$ and the $s$-th positive root of the equation $J_{1}(x)=0$ by $x_{8}{ }^{\prime}$. Since $J_{0}^{\prime}(x)=-J_{1}(x)$ this notation may be extended so that the $s$-th positive root of the equation $J_{0}^{\prime \prime}(x)=0$ is denoted by $x_{s}{ }^{\prime \prime}$, and so on. The $s$-th positive root of $J_{n}(x)=0$ will be denoted in a general way by $j_{n, 8}$ so that $j_{0,8}=x_{8}, j_{1,8}=x_{s}^{\prime}$.

$$
\begin{aligned}
J_{n}(x) & =I_{x}^{n} \text { (Bessel's table) } \\
& =I_{i x}^{n} \text { (HANSEN) }=J^{n}(x) \text { (LoMmeL). } \\
\Lambda_{n}(x) & =f_{n}(x) \text { (NYMTP) }=R_{n-i}\left(x^{2}\right) \text { (ChAPMAN) }
\end{aligned}
$$

$1 \mathrm{~F}$. W. BESSEL came upon these functions in discussion of planetary perturbations, and made the first tables of $J_{0}(x)$ and $J_{1}(x)$; Akad. d. Wissen., Berlin, Math. Klasse, Abh. for the year 1824, Berlin, 1826. The name "Bessel's function"' and the notation $J_{n}(x)$ are due to O. Schlömilch, $Z$. Math. Phys., v. 2, 1857.

2 D. Bernoulli, Akad. Nauk. Leningrad, Commentarii, v. 6 for the year 1732 and 1733, 1738, p. 116-119. See also G. A. MAGGr, Accad. d. Lincei, Atti, s. 3, Transunti, v. 4, 1880, p. 259-263; and M. Bôcher, New York Math. So., Bull., v. 2, 1893, p. 107-109.

$$
\text { A } . J_{0}(x) \text { and } J_{1}(x)
$$

1. 103-23D, HAYASHI $1, x=.01(.01) .05, .1(.1) .5,1,2,10(10) 50,100$

2. $21 \mathrm{D}$, AlDIS $2, x=0(.1) 6$

3. 20D, HAYASHI 1 , p. $115, x=1,10(10) 50,100$

4. $18 \mathrm{D}$, HaYASHI $3, x=0(.0001) .0055$

5. $18 \mathrm{D}$, HAYASHI $1, x=0(.001) .11$

6. $18 \mathrm{D}$, MeISSEL $1, x=1(1) 24$

7. 16D, HAYASHI $1, x=.12(.01) .5$

8. 15D, H. T. Davis, $x=15.5(.01) 25$

9. 15D, HAYASHI 1, p. $28, x=15.5(1) 24.5,25$

10. $14 \mathrm{D}$, Airey $17, x=9,10$

11. 12D, COMRIE, $x=0(.001) 16 .(.01) 25, \Delta^{2}$

12. $12 \mathrm{D}$, HAÝASHI $1, x=.5(.01) 25.1$ 
13. 12D, HAYASHI $3, x=25(.01) 25.51$

14. 12D, MEISSEL $1, x=0(.01) 15.5$

15. 12D, BAASMTC $6, x=0(.1) 25.5, \delta^{2}$

16. 10D, Airey 10, $x=6.5(.5) 16$; see no. 22

17. 10D, BESSEL, $x=0(.01) 3.2, \Delta^{2}$

18. 10D, BAASMTC $1, x=0(.001) 16(.01) 25, \delta^{2}$

19. 10D, NYMTP 3 , p. $2, x=0(.01) 10$

20. 9 duodecimals, TERRY, $x=0\left(12^{-2}\right) 1, \delta^{2}$

21. 7D, Watson $3, x=0(.02) 16$

22. 6D, Airey 10, $x=0(.2) 6$; see no. 16

23. 6D, P. A. HANSEN, $x=0(.1) 20$, with $J_{n}^{\prime}(x), \frac{1}{2} J_{n}{ }^{\prime \prime}(x), \frac{1}{6} J_{n}{ }^{\prime \prime \prime}(x), n=0,1$

24. 6D, HAMBURG, $x=0(.1) 15$

25. 6D, LOMMEL $2, x=0(1) 12$

26. $6 \mathrm{D}$, Meissel $3, x=1(1) 10,16,20$

27. $6 \mathrm{D} ; 4 \mathrm{D}$, FowLe, $x=0(.01) 4 ; 4(.1) 15$

28. 5D, WitkowskI, $x=0(.01) 15.09$

29. 5D, Corrington $\&$ Miehle, $x=m s, s=1(1) 20, m=0(1) 10$

30. 5D, Hayashi $2, x=0(.01) 16$

31. 5D, DALE, $x=0(.1) 15 ; 0(1) 20$

32. $4 \mathrm{D}$ or $4 \mathrm{~S}$, JAHNKE \& EMDE $1_{1}-1, x=0(.01) 15.5 ; 1_{1}-1_{2}, x=1(1) 24 ; 1,-16$, $x=1(1) 29$

33. $4 \mathrm{D}$, GLAZENAP, $x=0(.02) 16$

34. $4 \mathrm{D}$, McLachlan $2, x=0(.1) 15.9$

35. 4D, ClaPP, $x=25.3$ (mostly .3$) 39.9$

36. $4 \mathrm{D}$, BYERLY 1 and $2, x=0(.1) 15$

37. $4 \mathrm{D}$, MoRSE, p. $333, x=0(.1) .2(.2) 8$

38. 4D, Nicholson $2, x=.1, .4(.1) .6, .8(.1) 1.2,1.5(.5) 3(1) 6$

39. 5D, Corrington \& Miehle, $x=m s, s=1(1) 20, m=\pi(\pi) 5 \pi$

A2. $J_{0}(x)$, or $J_{1}(x)=-J_{0}^{\prime}(x)$; functions involving $J_{0}(x)$ and (or) $J_{1}(x)$

1. $20 \mathrm{D} ; 17 \mathrm{D}, J_{1}(x)$, HaYASHI 3 , p. $36, x=1,2 ; 3(1) 5$

2. $15 \mathrm{D}, \Lambda_{0}(x)=J_{0}(x)$, HAYASHI 1 , p. $28, x=15.5(1) 24.5,25$

3. $15 \mathrm{D}, J_{0}(x)$, NYMTP $1, x=0(1) 10$

4. $15 \mathrm{D}, J_{1}(x)$, HAYASHI $1, x=.1(.1) .5,15.5(.5) 25$

5. $14 \mathrm{D}, J_{0}(x)$, HAYASHI 1 , p. $119, x=22.9(.01) 23.1$

6. 7D, $J_{0}(40)$, HaMiltoN

7. $6 \mathrm{D}, J_{1}(x)$, STEINER, $x=20(.1) 31(.2) 41$

8. $5 \mathrm{D}, \log J_{0}(x)$ and $\log J_{1}(x)$, THÜRING, $x / a=1(1) 6, a=.21534$

9. $4 \mathrm{D}, J_{0}(x), \operatorname{ArRY} 4, x=0(.2) 10$

10. $6 \mathrm{D}, J_{0}(\pi x)$ and $\log \left|J_{0}(\pi x)\right|$, NAGAOKA $1, x=1(1) 50$

11. 5D, $J_{0}(\pi x)$, HaYASHI 2 , p. $99, x=1(1) 50$

12. $3 \mathrm{D}, J_{0}(\pi x)$, CoNRADY, $180 x=0(10) 600$

13. 3D, $J_{0}(\pi x)$, BuxToN, $180 x=600(10) 1040$

14. 3D, $J_{1}(\phi x)$, Condon, $x=.05, .1(.1) . \dot{3} ; \phi=\pi / 2,3 \pi / 2$

15. 3D, $1-J_{1}(2 x) / x$, MCLACHLAN $\&$ SowTER, $x=.5(.5) 10, .25, .75$

16. $6 \mathrm{D}, \Lambda_{1}(x)=2 J_{1}(x) / x$, LOMMEL 1 and $2, x=0(.1) 20$

17. 5D, $\Lambda_{1}(x)$, JAHNKE \& EMDE $1_{2}$ (p. 251), $1_{3}-1_{5}$ (p. 181), $x=0(.02) 9.98$

18. $4 \mathrm{D}, \Lambda_{1}(x)$, AIRY $1_{3}$ and $2, x=0(.2) 12$

19. $4-5 \mathrm{D}, \Lambda_{1}(x)$, SCHWERD, $180 x / \pi=$ [0(15)195; 4D], [210(15)1125, 1210(180)1930; 5D]

20. $4 \mathrm{D}, 10 \Lambda_{1}(x)$, CoNRADY, $180 x / \pi=0(40) 600$

21. $4 \mathrm{D}, S\left(\frac{1}{2} x\right)=2 \pi^{-\frac{1}{3}} \Lambda_{1}(x)$, HAMY $x=0(.2) 4.2$; compare VII

22. 4-5D, $\Lambda_{0}(x), \Lambda_{1}(x)$, JAHNKE \& EMDE, $1_{2}-1_{5}, x=0(.02) 9.98, \Delta$

23. $4 \mathrm{D}, 1-\Lambda_{1}(x)$, MORSE, p. 337, $x=0(.1) .2(.2) 4(.5) 10(1) 15$

24. $4 \mathrm{D}, \Lambda_{0}{ }^{2}(x)=J_{0}{ }^{2}(x$,$) AIRY 4, x=0(.2) 10$ 
25. $6 \mathrm{D}, \Lambda_{1}^{2}(x)=\left[2 J_{1}(x) / x\right]^{2}$, KÖHLER, $x=1(.02) 15.2$

26. $6 \mathrm{D}, \Lambda_{1}^{2}(x)$, LOMMEL 1 and $2, x=0(.1) 20$

27. $4-5 \mathrm{D}, \Lambda_{1}{ }^{2}(x)$, SCHWERD, $180 x / \pi=[0(15) 150 ; 4 \mathrm{D}]$, [165(15)1125, $\left.1210(180) 1930 ; 5 \mathrm{D}\right]$

28. $4 \mathrm{D}, \Lambda_{1}^{2}(x)$, PERNTER, p. $440, x=0(.2) 12$

29. $4 \mathrm{D}, \Lambda_{1}^{2}(x)$, CoNRADY, $180 x / \pi=0(40) 600$

30. $4 \mathrm{D}, \Lambda_{1}^{2}(x)$, GaNS $4, x=0(.5) 7$

31. $4 \mathrm{D}, \frac{1}{2} \Lambda_{1}^{2}(x)$, BoRN, p. $207, x=0(.2) 10$

32. $4 \mathrm{D}, \Lambda_{1}{ }^{2}(x), x=0(.2) 12$, BLUMER 3

33. 6-7S or D, $J_{1}\left(2 x^{3}\right) / x^{\ddagger}$, WARD, $x=[0(1) 5 ; 7 \mathrm{D}]$, [6(1)12,15; 6-7S]

34. 2-5D, $x J_{1}(x) / J_{0}(x)$, NANCARROW, $x=0(.01) .1(.05) 1.5$ (var.) 14.8. Also 7 graphs of this function with enlargement of details.

35. 5D, $1-J_{0}{ }^{2}(x)-J_{1}{ }^{2}(x)$, NAGAOKA $2, x=0(.2) 3.8$

36. 6D, $(4 / 3 x)\left[2 J_{1}(x)-J_{1}\left(\frac{1}{2} x\right)\right]$, LOMMEL $1, x=0(.2) 20$

37. $6 \mathrm{D},(1 / 6)\left[J_{0}(x)+2 J_{0}(2 x)+3 J_{0}(3 x)\right]$, LOMMEL $1, x=0(.2) 7.4,6.59$

38. 6D, $(2 / x) U_{2}(x, x), M^{2},(2 / x) V_{0}(x, x), M_{1}{ }^{2}$, Lommel 2, p. 284, 296, 323, 328, where $U_{1}(x, x)=V_{1}(x, x)=\frac{1}{2} \sin x, \quad U_{2}(x, x)=\frac{1}{2}\left[J_{0}(x)-\cos x\right], V_{0}(x, x)=\frac{1}{2}\left[J_{0}(x)+\cos x\right]$, $M^{2}=\left(U_{1}^{2}+U_{2}^{2}\right)(2 / x)^{2}, M_{1}^{2}=\left(V_{1}^{2}+V_{0}^{2}\right)(2 / x)^{2}$.

39. $20 \mathrm{D} ; 17 \mathrm{D}, J_{0}(x)-J_{2}(x)=2 J_{0}(x)-2 J_{1}(x) / x$, HAYASHI 3 , p. $36, x=1,2 ; 3(1) 5$

40. $3 \mathrm{D} ; 4 \mathrm{D}, 2 J_{1}{ }^{\prime}(x)=2\left[J_{0}(x)-J_{1}(x) / x\right]$, Nicholson $2, x=.5, .6, .8(.1) 1,1.2,1.5(.5) 3(1) 5$

41. $4 \mathrm{D}, J_{0}^{2}(x), x J_{0}^{2}(x), J_{1}^{2}(x), x^{-2} J_{1}^{2}(x), x^{-1} J_{1}^{2}(x),\left[J_{1}^{\prime}(x)\right]^{2}, x\left[J_{1}^{\prime}(x)\right]^{2}, J_{0}^{2}(x)-(4 / 3 x)-$ $J_{1}(x) J_{1}{ }^{\prime}(x), x J_{0}{ }^{2}(x)-(4 / 3) J_{1}(x) J_{1}{ }^{\prime}(x)$, CHREE, $x=0(.1) .2(.2) .8(.1) 1(.2) 3.8(.1) 4(.4)$ 4.8(.2)6 (var.) 14.8

B. Zeros: $x_{8}$ of $J_{0}(x)$, and $J_{1}\left(x_{8}\right)$

1. 10D, DAVIS \& KIRKHAM, $s=1(1) 150$

2. 10D, BAASMTC $1, s=1(1) 150$

3. 10D, 8D, Willson \& PeIRCE, $s=1(1) 40 ; x_{s}, 10 \mathrm{D} ; J_{1}\left(x_{s}\right), 8 \mathrm{D}$

4. $8 \mathrm{D}$, HAMBURG, $s=1(1) 40$

5. 7D, Airey 17 (Willson \& PeirCe), $s=1(1) 40$

6. $6 \mathrm{D}$, PEIRCE $1, s=1(1) 12$

7. $6 \mathrm{D}, 4 \mathrm{D}$, FowLE, $s=1(1) 10 ; x_{s}, 6 \mathrm{D} ; J_{1}\left(x_{s}\right), 4 \mathrm{D}$

8. 5D, HAYASHI $2, s=1(1) 60$

9. 5D, KALÄHNE $1, s=1(1) 6$

10. $4 \mathrm{D}$ or $4 \mathrm{~S}$, JAHNKE \& EMDE $1_{1}-1_{6}, s=1(1) 40$

\section{B. Zeros: $x_{\text {, only }}$}

1. 10D, MeIsSEL $1, s=1(1) 10$

2. 7D, Watson $3, s=1(1) 40$

3. $6 \mathrm{D}$, PEIRCE $2, s=1(1) 10$

4. $5 \mathrm{D}$, GlazenaP, $s=1(1) 40$

5. $5 \mathrm{D}$, WiTKOWSKI, $s=1(1) 10$

6. 5D, SMith, Rodgers, \& Traub, $s=1(1) 8$

7. $5 \mathrm{D}, \mathrm{KALÄHNE} 2$, p. $72, s=1$ (1)6

8. $4 \mathrm{D}$, Airey $19, s=1(1) 10$

9. $4 \mathrm{D}$, ByerLy $2, s=1(1) 10$

10. $3 \mathrm{D}$, BOURGET, $s=1(1) 9$

11. $4 \mathrm{D}, x_{8} / \pi$, STOKES, $s=1(1) 12$

12. $4 \mathrm{D}, x_{s} / \pi$, MORSE, p. $153, s=1(1) 3$

\section{B. Zeros: functions of $x_{8}$}

1. 10D; 9D, $\log x_{b} ; \log \left|J_{1}\left(x_{s}\right)\right|$, DAvis \& KIRKHAM, $s=1(1) 150$

2. 10D; 7D, $\log x_{s} ; \log \left|J_{1}\left(x_{s}\right)\right|$, Willson \& PEIRCE, $s=1(1) 40$

3. 3D, $x_{s-1} / x_{s}$, BOURGET, $s=2(1) 9$

4. $4 \mathrm{D}, x_{s+w} / x_{1}, x_{s+w}-x_{1}, \operatorname{KALÄHNE} 1, s=1,2, w=1(1) 4$; and $s=3, w=1(1) 3$ 
5. 4D, For 24 equally spaced intervals, from 0 to $x_{s}$, abscissae are calculated; for each $x$ corresponding ordinates $x J_{1}(x)$ are tabulated in four tables, $s=1(1) 4$, PERRY \& HUNT

6. 5S, $\sum_{s=8}^{\infty}\left(x_{s}\right)^{-2 m}$, BUTTERWORTH $1, m=2(1) 10$

7. $\sum_{s=1}^{10} x_{s}^{-4}=0.031246, \sum_{s=1}^{\infty} x_{s}^{-4}=1 / 32$, and $\sum_{s=1}^{\infty} x_{s}^{-2}=1 / 4$, MCLEOD

8. Exact numerical values for $\sum_{s=1}^{\infty} 1 / x_{s}^{p}$, RAYLEIGH, $p=2(2) 10$; also $p=16$ (CAYLEY)

9. Exact numerical values for the following summation among others:

$\sum_{s=1}^{\infty} 1 / x_{s}^{p},(p=2,4,6) ; \sum_{s=1}^{\infty} 1 /\left[x_{s}^{p} J_{1}\left(x_{s}\right)\right](p=3,5,7) ; \sum_{t=1}^{\infty} 1 /\left[x_{s}^{p} J_{1^{2}}\left(x_{s}\right)\right],(p=4,6,8)$, FORSYTH

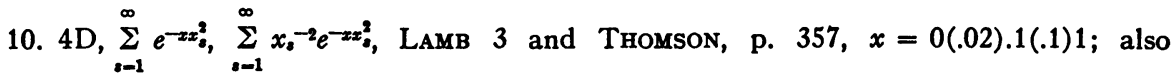
$\sum_{i=1}^{\infty} x_{t}^{-2}=1 / 4$

11. 3D, $1-4 \sum_{t=1}^{\infty} e^{-x_{s}^{2} t} ;$ HILL, $t=.003(.002) .007, .01(.01) .05, .07, .1(.05) .2(.1) .5, .7,1$

12. $4 \mathrm{D},\left(32 / \pi^{2}\right) \sum_{n=0}^{\infty} \sum_{s-1}^{\infty} 1 /\left[\left(n x_{s}\right)^{2}(2 n+1)^{4}+x_{s}^{4}(2 n+1)^{2} z\right]$, STADIE, $z=0, .04, .25,1,4,25, \infty$

13. $5 \mathrm{D}, \sum_{s=1}^{\infty} 2 e^{x_{s}^{2} \theta} /\left[x_{s} J_{1}\left(x_{s}\right)\right]$, OLsON \& Schultz, $\theta=.001(.001) 4$

14. 3D, $1-4 \sum_{s=1}^{\infty} x_{s}^{-2} \cdot e^{-0 x_{s}^{2}}$, BUCHWALD, $g=.01(.01) .1(.02) .2(.05) .8$

15. $4 \mathrm{D}, \sum_{s=1}^{\infty} J_{0}\left(r x_{s}\right) e^{-\varepsilon x_{0}} /\left[x_{s}^{n} J_{1}\left(x_{s}\right)\right]$, BeRTRAM, $r=0(.1) .9 ; n=1, z=0(.05) 1.75 ; n=2$, $z=0(.05) 1.5 ; n=3,4, z=0(.05) 1$

16. $4 \mathrm{D}, \log \left[J_{1}\left(\frac{1}{1} x_{s}\right) / J_{1}\left(x_{s}\right)\right]$, PEIRCE $1, s=1(1) 12$

17. $4 \mathrm{D}, y=\sum_{s=1}^{\infty}\left[J_{1}\left(\frac{1}{2} x_{s}\right) e^{-x_{s}^{2} t} / J_{1}\left(x_{s}\right)\right]$, PeIRCE 1, p. 61, $t=.17, .25$ (var.) $1.5(.5) 3(1) 6,8$

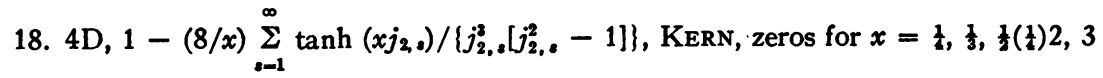

See also XII A

B. Zeros: $x_{s}^{\prime}$ of $J_{1}(x)$, and $J_{0}\left(x_{s}{ }^{\prime}\right)$

1. $16 \mathrm{D}$, MEISSEL $2, s=1(1) 50$

2. 10D, Davis \& KIRKHAM, $s=1(1) 150$

3. 10D, BAASMTC $1, s=1(1) 150$

4. 8D, HAMBURG, $s=1(1) 50$

5. 7D, AIREY $17, s=1(1) 40$

6. $6 \mathrm{D}$, FoWLE, $s=1(1) 15$

7. $5 \mathrm{D}, \mathrm{HAYASHI} 2, s=1(1) 60$

8. $4 \mathrm{D}$ or $4 \mathrm{~S}$, JAHNKE \& EMDE $1_{1}-1_{5}, s=1(1) 50$

9. $4 \mathrm{D}, \mathrm{MORSE}, \mathrm{p} .153-155, s=1(1) 3$

10. 3D (mostly), MALOW, $s=1(1) 5(5) 10(10) 40$

\section{$B_{5 .}$ Zeros: $x_{a}^{\prime}$ only}

1. 7D, WATSON $3, s=1(1) 40$

2. $6 \mathrm{D}$, LOMMEL 1 , p. 167 and 2, p. $315, s=1$ (1)6

3. $6 \mathrm{D}$, Gray \& Mathews $1_{1}-1_{2}, s=1$ (1)6

4. 5D. KALÄHNE 2, p. 72, $s=0(1) 5$ 
5. $4 \mathrm{D}$, Glazenap, $s=1(1) 40$

6. $4 \mathrm{D}$, BYERLY $2, s=1(1) 10$

7. $4 \mathrm{D}$, Airey $19, s=1(1) 10$

8. $4 \mathrm{D}$, SMith, Rodgers, \& Traub, $s=1$ (1) 9

9. $3 \mathrm{D}$, BOURGET, $s=1(1) 9$

10. $6 \mathrm{D}, x_{s}^{\prime} / \pi$, LOMMEL 1 and $2, s=1(1) 6$

11. $4 \mathrm{D}, x_{s}^{\prime} / \pi$, STOKES, $s=1(1) 12$

12. $4 \mathrm{D}, x_{s}^{\prime} / \pi$, MORSE, p. $153, s=1(1) 3$

13. 3D, $x_{s}^{\prime} / 2 \pi$, PERNTER, $s=1(1) 9$

14. $1 \mathrm{D}, 180 x_{s}^{\prime} / \pi$, SCHWERD, $s=1(1) 6$

\section{B. Zeros: functions of $x_{s}^{\prime}$}

1. $10 \mathrm{D} ; 9 \mathrm{D}, \log x_{s}^{\prime} ; \log \left|J_{0}\left(x_{s}^{\prime}\right)\right|$, Davis \& KrRKHAM, $s=1(1) 50$

2. $4 \mathrm{D}, x_{s+w}^{\prime} / x_{1}^{\prime}, x_{s+w}^{\prime}-x_{1}^{\prime}$, KALÄHNE $1, s=1,2, w=1(1) 4$; and $s=3, w=1(1) 3$

3. $3 \mathrm{D}, x_{s-1}^{\prime} / x_{s}^{\prime}$, BOURGET, $s=2(1) 9$

4. $5 \mathrm{D}, I_{s}=1-J_{0}^{2}\left(x_{s}{ }^{\prime}\right)-J_{1}^{2}\left(x_{s}\right)$, and $I_{s}-I_{s-1}$, NAGAOKA $2, s=1(1) 10(10) 50$

5. $5 \mathrm{D}, \sum_{s=3}^{\infty}\left(x_{s}^{\prime}\right)^{-2 m}$, BUTTERWORTH $1, m=2(1) 10$

6. Exact values of $\sum_{s=1}^{\infty} 1 /\left[\left(x_{s}^{\prime}\right)^{p} J_{0}\left(x_{s}^{\prime}\right)\right], \quad(p=2,4,6) ; \sum_{s=1}^{\infty} 1 /\left[\left(x_{s}^{\prime}\right)^{p} J_{0}^{2}\left(x_{s}^{\prime}\right)\right], \quad(p=2,4,6)$; $\sum_{s=1}^{\infty} 1 /\left[\left(x_{s}^{\prime}\right)^{p} J_{0}^{3}\left(x_{s}^{\prime}\right)\right],(p=6,8)$, FORSYTH

7. 5D, $\sum_{s=1}^{\infty}\left(x_{s}^{\prime}\right)^{-2} J_{0}\left(x x_{s}^{\prime}\right) e^{-y x_{s}^{\prime}}{ }^{2} / J_{0}\left(x_{s}^{\prime}\right)$, Newman \& ChURCH, $x=.1 ; y=0, .005, .01(.01)$ $.1(.02) .2(.05) .5,1$

See also XII A

\section{B. Zeros: functions of $J_{0}(x)$ and $J_{1}(x)$}

1. 3D, $J_{0}(x)-.3,2$ zeros, Prescott, p. 486

2. $3 \mathrm{D}, x J_{0}(x)-.75 J_{1}(x)$, a zero, Prescott, p. 488.

3. $4 \mathrm{D}, x J_{0}(x)-k J_{1}(x), k=\frac{1}{2}, \frac{2}{3}$, first 10 zeros, Airey 5

4. $3 \mathrm{D}, x J_{0}(x)+399 J_{1}(x)$, a zero, LAMB 4

5. $6 \mathrm{D}$, first 6 max. and min. of $2 J_{1}(x) / x$ and of $\left[2 J_{1}(x) / x\right]^{2}$, LOMMEL 1, p. 167 , i.e., values of 6 zeros of $x J_{0}(x)-2 J_{1}(x)\left[=-x J_{2}(x)\right]$

6. 3-5D, $x J_{0}(x)-2 J_{1}(x)$, first 12 zeros, SCHWERD; $180 x / \pi=0,219.6,401.9, \cdots 1750$, 1930

7. $3 \mathrm{D}, x J_{0}(x)-2 J_{1}(x)$, first 15 zeros $\left(j_{2,8} / 2 \pi\right)$, MASCART, p. 312

8. 3D, $x J_{0}(x)-2 J_{1}(x)$, first 18 zeros $\left(j_{2,8} / 2 \pi\right)$, Pernter, p. 542

9. $4 \mathrm{D}, 3 x J_{0}(x)-2 J_{1}(x)$, first 10 zeros, Airey 5

10. $4 \mathrm{D}, 3 x J_{0}(x)-2 J_{1}(x)$, first 8 zeros, Ruedy 1

11. $4 \mathrm{D}, 2 x J_{0}(x)-J_{1}(x)$, first 10 zeros, Airey 5

12. $3 \mathrm{D}, x J_{0}(x)-2 J_{1}(x)$, first 4 zeros, Petrž́ĺlkA

13. $3 \mathrm{D}, J_{0}(x)-m x J_{1}(x)$, first 8 zeros, $m=.32 ; 6$ zeros, $m=.427 ; 3$ zeros, $m=1.27$; 1 zero, $m=4.133$, Sснміск

14. 5D; 3D, $J_{0}(x)-10 x J_{1}(x)$, first zero; next 4 zéros, PeIrCe 2

15. 6D; $2 \mathrm{D}, J_{0}(x)-1000 x J_{1}(x)$, first zero; second zero, PEIRCE 2

16. 6D, $J_{0}(x)-100000 x J_{1}(x)$, first zero, PEIRCE 2

17. $4 \mathrm{D}, 5 J_{0}(x)-x J_{1}(x)$, first 5 zeros, PeIrCe 1

18. $4 \mathrm{D}, 25 J_{0}(x)-2 x J_{1}(x)$, first 5 zeros, PEIRCE 1

19. 5D, $\left(1-1.3666 x^{2}\right) J_{0}(x)-1000 x J_{1}(x)$, first zero, PEIRCE 3

20. 4D, $\left(1-0.01366 x^{2}\right) J_{0}(x)-10 x J_{1}(x)$, first 3 zeros, PeIRCE 3

21. $2 \mathrm{D}, 3 x J_{0}^{2}(x)-6 J_{0}(x) J_{1}(x)+x J_{1}^{2}(x)$, first zero, Poncin

22. $3 \mathrm{D}, J_{1}{ }^{\prime}(x)=J_{0}(x)-J_{1}(x) / x$, first 4 zeros, ChreE 
23. 3D, $J_{1}^{\prime}(x)$, first 3 zeros, Schelkunoff, p. 323

24. $3 \mathrm{D}, 3 x J_{1}^{\prime}(x)+J_{1}(x)$, first 4 zeros, ChreE

25. $3 \mathrm{D}, 2 x J_{1^{\prime}}(x)+J_{1}(x)$, first 4 zeros, Chree

26. $4 \mathrm{D}, x J_{1}^{\prime}(x)+\left(1-2 x^{2}\right) J_{1}(x)$, first 2 zeros, ChreE

27. 3D, $(m-2) J_{1}(x)-(m-1) x J_{2}(x)=(m-1) x J_{0}(x)-m J_{1}(x), 3$ zeros each for $m=3$, $4 ; 2$ each $m=2.22,2.50,3.33$, RUEDy 2

28. $5 \mathrm{D},(x-1)^{2} \phi(h a)-(\beta x-1)[x-\phi(k a)]=0, \phi(y)=y J_{0}(y) / J_{1}(y), h=\gamma(\beta x-1)^{4}$ $k=\gamma(2 x-1)^{3}, x=\left(v / v_{0}\right)^{2}(1+\sigma), \beta=(1-2 \sigma) /(1-\sigma), d / L=a \gamma / \pi$, BANCROFT, table of $v / v_{0}$ for $d / L=0(.05) 1(.2) 2, \infty ; \sigma=.1(.05) .4$. Also graphs of $v / v_{0}$ as functions of $d / L=0(.2) 2.6 ; \sigma=0(.1) .5$

$$
\mathrm{C}_{1}, J_{n}(x) \text {, including } n>1
$$

1. 103-10D, HAYASHI 1

$\begin{array}{llllllllc}x & n & D & x & n & D & x & n & D \\ .01 & 0-7 & 40-21 & .2 & 0-11 & 40-20 & 10 & 0-50 & 65-30 \\ .02 & 0-8 & 42-21 & .3 & 0-12 & 47-20 & 20 & 0-56 & 45-20 \\ .03 & 0-9 & 42-22 & .4 & 0-14 & 42-20 & 30 & 0-69 & 36-18 \\ .04 & 0-9 & 42-20 & .5 & 0-14 & 35-20 & 40 & 0-82 & 36-18 \\ .05 & 0-10 & 40-20 & 1 & 0-32 & 98-46 & 50 & 0-88 & 31-15 \\ .1 & 0-10 & 43-21 & 2 & 0-40 & 103-48 & 100 & 0-135 & 24-10\end{array}$

2. $18 \mathrm{D} ; 20 \mathrm{D}, J_{n}(x)$, HAYASHI 3 , p. $34, n=0(1) 2 ; 3(1) 6, x=0(.0001) .0055$

3. 20D, $J_{20}(20)$, MEISSEL 3

4. 18D, MEISSEL $5, x=0(1) 24, n=0(1) N$

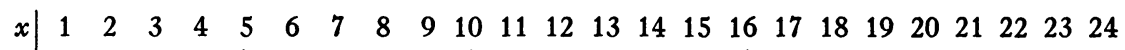
\begin{tabular}{l|llllllllllllllllllllllll}
$N$ & 16 & 19 & 22 & 25 & 27 & 30 & 32 & 34 & 35 & 37 & 39 & 41 & 43 & 44 & 46 & 47 & 49 & 50 & 52 & 54 & 55 & 57 & 58 & 60
\end{tabular}

5. 12D, BAASMTC $6, n=0(1) 20, x=0(.1) 25.5$, with varying differences as needed

6. 10D, BAASMTC $2, n=0(1) 20, x=0(.1) 25$

7. $10 \mathrm{D}$, AIREY $10, n=0(1) 13, x=6.5(.5) 16$

8. 8D; 10D; 12D, MeISSEL $4, n=10(1) 14 ; 15(1) 19 ; 20,21$ [table of $J_{2 n}(n)$ ]

9. 8D, BAASMTC $2, n=2(1) 12, x=0(.01) 10, \delta_{m}^{2}$

10. $8 \mathrm{D}$, MeISSEL $3, J_{n}(1000), n=967,968,981(1) 1000$

11. 7D, P. A. HANSEN $n=5,6, x=0(.2) 2 ; n=8,9, x=2(.2) 4 ; n=11,12, x=4(.2) 6$; $n=13,14, x=6(.2) 8 ; n=15,16, x=8(.2) 10 ; n=18,19, x=10(.2) 12 ; n=20$ $21, x=12(.2) 14 ; n=23,24, x=14(.2) 16 ; n=25,26, x=16(.2) 18 ; n=27,28$, $x=18(.2) 20$, with $J_{n}^{(p)}(x) / m !, p=m=1 ; p=m=2 ; p=m=3$

12. 7D, Watson $3, n=2(1) 5, x=0(.1) 5$

13. 7D, $J_{n}\left(x_{s}\right)$, BuchHolz $3, n=1(1) 10, s=1 ; n=1(1) 16, s=2 ; n=1(1) 20, s=3$

14. $6 \mathrm{D}$, Airey $10, n=0(1) 13, x=0(.2) 6$

15. $6 \mathrm{D}$, LOMMEL 2 , p. $315-317, n=0(1) 20, x=0(1) 12$

16. $6 \mathrm{D}$, Watson $3, n=0(1) 20, x=0(1) 12$

17. 6D, MeISSEL 3, $n=0(1) m, 7 \leq m \leq 35, x=1(1) 10,16,20$

18. 6D, AIREY 13B, $n=100(1) 109, x=109$

19. $5 \mathrm{D}$, DALE, $n=0(1) 12, x=0(1) 20$

20. 5D, TVERITIN, $n=2, x=0(.01) 10$

21. $4 \mathrm{~S}$, JahnKe \& EMdE $1_{1}-15, n=0(1) m, 17 \leq m \leq 61 ; x=1(1) 24 ; \ngtr 18 \mathrm{D}$. Compare no. 4 above

22. 4S, JAHNKE \& EMDE $1_{3}-15, n=0(1) m, 42 \leq m \leq 44 ; x=25(1) 29 ; \ngtr 6 \mathrm{D}$

23. $4 \mathrm{~S}, J_{10}(x)$, FoK, $x=0(1) 24$

24. 4D, Nicholson $2, n=0(1) 3, x=.1, .4(.1) .6, .8(.1) 1$; and $n=0(1) 8, x=1.1,1.2$, $1.5(.5) 3(1) 6$

25. $4 \mathrm{D}$, Mclachlan 2 , p. $175, n=2(1) 4, x=0(.1) 4.9$

26. $4 \mathrm{D}, \mathrm{MORSE}$, p. $333, n=2, x=0(.1) .2(.2) 8$ 
27. 7D, WATson $3, n=x=1(1) 50$

28. 6D, AireY 13A, $n=x, x-1 ; x=1(1) 50(5) 100(10) 200(20) 400(50) 1000(100) 2000$ (500)5000(1000)20000(5000)30000(10000)50000, $100000,500000,1000000$

29. $6 \mathrm{D}$, AIREY $31, n=x, x \pm 1 ; x=1(1) 20$

30. 6D, Airey $20, n=x, x-1 ; x=0(1) 10$

31. $J_{6}(6)$ to $6 \mathrm{D}, J_{48}(48)$ to $9 \mathrm{D}, J_{760}(750)$ to $14 \mathrm{D}$, AIREY $13 \mathrm{~B}$

32. 4D, $[J(x) / x]^{2}, 2 J_{1}(x) J_{2}(x) / x, 2 J_{1}(x) J_{2}(x) / x^{2}$, BORN, p. 207, $x=0(.2) 10$, with graphs

33. $3 \mathrm{D}, J_{n}{ }^{2}(x), n=0(1) 14$, RowlaND, $x=0(.2) 1(1) 6,8,10$, and 2.605, 3.832, 5.52, 7.016, 8.654

34. 3D; $2 \mathrm{D}, J_{1}\left(j_{2, s}\right) ; j_{2, s}, J_{0}\left(x_{1}{ }^{\prime}\right) / J_{1}\left(j_{2, s}\right), J_{0}\left(j_{1,2}\right) / J_{1}\left(j_{2, s}\right)$, MALOW, $s=1(1) 5,10(10) 40$

\section{Functions, derivatives, and differences of $J_{n}(x)$}

1. $7 \mathrm{D}, n^{1 / 3} J_{n}(n)$, WaTSON $3, n=1(1) 50$

2. 5D, $\log J_{n}(x)$, THÜRING, $n=0(1) 3 ; x / a=1(1) 6, a=.21534$

3. 5S, $x^{2 n} J_{2 n}(x) / n$ !!, and $x^{2 n+1} J_{2 n+1}(x) /(n+1)$ !!, PICHT, $x=1(1) 15$

4. 6D, $(1 / n !)(x / 2)^{n} / J_{n}(x)=\sum_{k=0}^{\infty} a_{n, k}(x / 2)^{2 k},(1 / n !)(x / 2)^{n} J_{n-1}(x) / J_{n}(x)=\sum_{k=0}^{\infty} b_{n, k}(x / 2)^{2 k}$, $|x / 2|<1$, BRIXY 1 , tables of $a_{n, k}, b_{n, k}, n=0(1) 3, k=0(1) 9$. It is shown that if $c_{m, k}$ be the coefficients of similar expansion of $(1 / n !) J_{n+1}(x) / J_{n}(x), c_{n, k}=-b_{n_{k} k+1}$

5. $(16-p) \mathrm{D}, J_{0}^{(p)}(x)$, HAYASHI 1 , p. $28, \mathrm{p}=1(1) 10$ for $x=.1(.1) .5$, and $p=1(1) 9$ for $x=16(.5) 25$

6. $\gtreqless 12 \mathrm{D}, J_{0}^{(p)}(x), J_{1}^{(p)}(x)$, NYMTP $5, x=0(.1) 10$

7. $\leq 14 \mathrm{D}, J_{n}^{(p)}(0)$, HaYASHI 3 , p. $29, n=0(1) 5, p=1(1) 14$

8. $20 \mathrm{D} ; 17 \mathrm{D}, 2 J_{n}^{\prime}(x)$, HAYASHI 3 , p. $36, n=1(1) 27, x=1,2 ; 3(1) 5$

9. 5-2S, $J_{n}(p), J_{n}^{\prime}(p)$, SEITZ $1, p=.02095, n=[0,1 ; 5 S],[2 ; 4 S],[3 ; 2-4 S] ; p=.0000419$, $n=0$ (exact), $[1 ; 4 S],[2 ; 2 S]$

10. $4 \mathrm{D}, 2 J_{n}{ }^{\prime}(x)$, NICHOLSON $2, n=1(1) 6, x=1.2,1.5(.5) 3(1) 5$

11. $3 \mathrm{D}, 2 J_{n}{ }^{\prime}(x)$, Nicholson $2, n=1(1) 3, x=.5, .6, .8(.1) 1$

12. $4 \mathrm{D}, T\left(\frac{1}{2} x\right)=8 J_{2}(x) \pi^{-\frac{1}{3}} /\left(3 x^{2}\right)$, HAMY $x=0(.2) 4.2$; compare VII

13. 7D, $J_{n}{ }^{\prime}(n)$, and $n^{2 / 3} J_{n}{ }^{\prime}(n)$, WATSON $3, n=1(1) 50$

14. 19 or $20 \mathrm{D}, \Delta^{p} J_{n}(x)$, HAYASHI 3 , p. $30, p=1(1) 4 ; n=0(1) 6 ; x=0(.0001) .0055$

See also VII

\section{$D_{1}$. Zeros: $j_{n, 8}$ including $n>1$}

1. 12D, BAASMTC $9, n=0$ (1) 20, about 52 zeros, $x \ngtr 25$

2. 7D, WATSON $3, n=0(1) 5, s=1(1) 40$

3. $6 \mathrm{~S}$, Airey $14, n=10,100,1000, s=1(1) 5$

4. 6S (mostly), AIREY 19, $n=0(1) 10(5) 20(10) 50(25) 100(100) 500(250) 1000, s=1$ (1) 10

5. $J_{1000}(x)$ has 218 zeros between 1000 and 2000 , WATson 1

6. $6 \mathrm{D}$, LOMMEL $2, n=2, s=1(1) 5$

7. 5D (mostly), $4 \mathrm{D}$, Smith, Rodgers, \& Traub, $n=0(1) 19, s=1(1) 8$

$$
\begin{array}{l|llllllllllrrrrr}
n & 0 & 1 & 2 & 3 & 4 & 5 & 6 & 7 & 8 & 9 & 10 & 11 & 12 & 13-15 & 16-19 \\
s & 8 & 7 & 7 & 6 & 6 & 5 & 5 & 5 & 4 & 4 & 4 & 4 & 3 & 2 & 1
\end{array}
$$

8. $5 \mathrm{D}$, WitkowskI, $n=2, s=1(1) 5$; also $4 J_{1}^{2}\left(j_{2,8}\right) / j_{2, \text { e }}^{2}$,

9. 5D, IKEDA and HAYASHI 2, p. $98, n=1000, s=1$

10. $4 \mathrm{D}$, JAHNKE \& EMDE $1_{1}-1_{5}$ (p. 206), for $k=\infty, n=0,1,2, s=1$ (1)6 (suggested by the Liverpool Index)

11. 3D, BOURGET, $n=0(1) 5, s=1(1) 9$

12. $3-5 \mathrm{~S}, j_{2, s}, j_{2,8}^{3}, j_{2,8}^{2}-1, \mathrm{KERN}, s=1(1) 5$

13. 3D, GANS $2, n=2,3 ; s=1,2$

14. 2D, AIREY 13A, p. 13, and 13B, p. 52, $n=1000, s=1$

15. 2D, RaMachandRaN, $n=2, s=1(1) 5$; also $J_{1}\left(j_{2,8}\right) / j_{2}$, to $3 \mathrm{D}$ 


\section{$D_{2}$ Zeros: $j_{2,8} / \pi$, and miscellaneous}

1. $6 \mathrm{D}, j_{2,8} / \pi, 2 J_{1}\left(j_{2, s}\right) / j_{2, s}, 4 J_{1}{ }^{2}\left(j_{2, s}\right) / j_{2}{ }^{2}, 8$, LOMMEL 1 and $2, s=1(1) 5$

2. 5D, $4 J_{1}^{2}\left(j_{2, s}\right) / j_{2, s}^{2}$, Pernter, p. 442 , and MASCART, p. $312, s=1(1) 9$

3. $5 \mathrm{D}, 2 J_{1}\left(j_{2, s}\right) / j_{2, s}$ and $4 J_{1}{ }^{2}\left(j_{2, s}\right) / j_{2, s}^{2}$, SCHWERD, p. [146], $s=7(1) 11$

4. $4 \mathrm{D}, j_{2, s} / \pi, s=1(1) 3$, and $J_{1}\left(j_{2, s}\right), s=1(1) 4$, MORSE, p. 153-155

5. 3D; 5D, $j_{2, s} / 2 \pi, \Delta ; 4 J_{1}{ }^{2}\left(j_{2, s}\right) / j_{2, s}^{2}$, Pernter and Mascart, $s=1(1) 9$

6. $3 \mathrm{D}, j_{2, s} / \pi$, WRINCH, $s=1(1) 9$

7. $2-3 \mathrm{D}, x_{s}^{\prime}, J_{0}\left(x_{s}{ }^{\prime}\right), j_{2, s}, J_{1}\left(j_{2,8}\right), J_{0}\left(x_{1}{ }^{\prime}\right) / J_{1}\left(j_{2, s}\right), J_{0}\left(j_{1,2}\right) / J_{1}\left(j_{2, s}\right)$, MALOW, $s=1(1) 5$, $10(10) 40$

8. $4 \mathrm{D}, J_{n}{ }^{\prime}(x)$, SMith, Rodgers, \& TraUb, zeros $<25 ; p=$ number of zeros

$$
\begin{array}{r|rrrrrrrrrrrrrrrrrrr}
n & 0 & 1 & 2 & 3 & 4 & 5 & 6 & 7 & 8 & 9 & 10 & 11 & 12 & 13 & 14 & 15 & 16 & 17 & 18-22 \\
p & 9 & 8 & 7 & 7 & 6 & 6 & 5 & 5 & 5 & 4 & 4 & 3 & 3 & 3 & 2 & 2 & 2 & 2 & 1
\end{array}
$$

9. $3 \mathrm{D}, \mathrm{J}_{n}{ }^{\prime}(x)$, Thomson (RAyleigh) p. $347, n=2,3, s=1(1) 3$

10. $4 \mathrm{D}, x^{2} J_{0}(x)+k J_{2}(x), 4$ zeros $(x / \pi)$ for each $k$, MORSE, p. $159, k=0(.5) 1(1) 6(2) 10$

11. $4 \mathrm{D}, 9 x J_{n}{ }^{\prime}(9 x) J_{n}(x)-x J_{n}(9 x) J_{n}{ }^{\prime}(x), n=0,1$ (4 zeros), 2,3(3 zeros),4(2 zeros), ThILо, p. 557

12. 3D (mostly), $2 \pi\left|x J_{n+1}^{2}(x)\right|-1$, BorN \& Fuchs, $n=-1(1)+7, s=0(1) 11 ; 8$ pure imaginary, and 39 real zeros

13. $2 \mathrm{D},\left|2 \pi x\left[J_{n}^{2}(x)+J_{n+1}^{2}(x)\right]\right|-1$, Fuchs, $n=0(1) 7$; one pure imaginary, and one real zero, for each $n$

14. 2D, $J_{2}(x)-a^{2} J_{2}(a x)$, STEWARD, p. $171, a=0, \frac{1}{2}$, 1, first zeros

15. $2 \mathrm{D}, x J_{7}(x)-2 J_{6}(x)$, LAMB 6 , first zero

\section{E. Graphs, reliefs}

1. $J_{0}(x), J_{1}(x), 0<x<18 ; J_{n}(1), J_{n}(10) ; J_{n}(50)$, and $J_{n}(100)$, HAYASHI 1 , p. 1 ; p. 49 ; 58-59

2. $J_{0}(x), J_{1}(x), 0<x<25, \mathrm{~K}_{\mathrm{ALÄHNE}} 3$

3. $J_{0}(x), J_{1}(x) ; 0<x<10, \mathrm{DAHL}$

4. $1-J_{1}(2 x) / x, 0<x<14$, A. H. Davis

5. $J_{n}(x), n=0(1) 4 ; x=0(1) 20$, SCHRÖDINGER

6. $J_{n}(x), n=0(1) 12,18 ; x=0(1) 17$, HAGUE

7. $J_{n}(x)=$ const., $-4<n<+8 ; 0<x<18$, JAhNKE \& EMde $1_{2}$ (p. 222, fig. 114), $1_{3}-1_{5}$ (p. 152 , fig. 84 ); see no. 17

8. $J_{n}(n x), n=1(1) 3,0<x<30$, D. L. WeBsteR

9. $J_{n}(x)$ and $-J_{n}^{\prime}(x), n=0(1) 5 ; 0<x<18$, Colwell \& HaRdY

10. $J_{n}(n+1), J_{n}(n), 2 J_{n}^{\prime}(n), 10<n<1000$, JAHNKE \& EMDE, $1_{3}-1_{5}$ (p. 170, fig. 95)

11. $(2 n+2) J_{n+1}(x) / x, n=0(2) 6$, ZERNIKE, $0<x<13$

12. Bessel functions of large order: steepness at the zeros, and extreme values, $4<n<5000$, JAHNKE \& EMDE $1_{2}$ (p. 240, figs. 125-126), $1_{3}-1_{5}$ (p. 169, figs. 92-93)

13. $j_{m,}-n$ as function of $n, 0<n<1000$, JAHNKE \& EMDE $1_{2}$ (p. 234-236, figs. 120, 121, 123), $1_{3}-1_{5}$ (p. $162-165$, figs. $87,88,90$ )

14. Model of $\left|J_{n}(x) / x\right|$ for positive integral values of $n$, Schouten

15. $2 n^{2} J_{n}^{2}(n x), n=100$, FOK \& KolPINSKY, $1<x<1.3$

16. Relief of $z=J_{n}(x), 0<n<8,0<x<16$, showing lines of zeros, McLachlan 2, p. 21

17. Relief of $z=J_{n}(x), 0<n<10,0<x<20$, showing curves $J_{n}(x)=$ const. on the surface, JAHNKe \& EMDE $1_{2}$ (p. 223, fig. 115), $1_{3}-1_{5}$ (p. 153, fig. 85); see no. 7

18. Relief of $z=J_{n}(x),-2<z<+2,-4<n<+10,0<x<14$, JAHNKE \& EMDE $1_{2}$ (p. 192, figs. 92-93), $1_{3}-1_{5}$ (p. 126, figs. 67-68)

19. $J_{1}(x) / x, 0<x<20$, RAMACHANDRAN

20. $y=\sum_{s=1}^{\infty} e^{-k x_{0}{ }^{2} t}$, EURINGER, $k=1.10^{-8}$

21. $J_{1}(x \sin \phi) / x \sin \phi, x=5,10 ; \phi=-90^{\circ}\left(10^{\circ}\right)+90^{\circ}$, McLaChlan 2, p. 50

22. $J_{0}(x) / J_{1}(x), 0<x<3$, LÜDI 
23. $x=J_{0}(y) / J_{1}(y), x=-1(.2)+1,1 / x=-1(.2)+1$, JAHNKE $\&$ EMDE $1_{2}$ (p. 270 , fig. 139), $1_{3}-1_{5}$ (p. 200, fig. 106)

24. $\left[J_{1}(x) / x\right]^{2}, 2 J_{1}(x) J_{2}(x) / x, 2 J_{1}(x) J_{2}(x) / x^{2}$, BoRN, p. 208; see I $\mathrm{C}_{1} 32$

25. $y=\left[J_{1}^{2}(x)-J_{0}(x) J_{2}(x)\right] / J_{0}^{2}(x), 0<x<8.5$; also magnified $0<x<1.6$, TuRTon. It is also shown that the equation $y=z$ has but one positive root when $z=.9397$; when $z$ rises from .9397 to 1 there are successively $3,5,7, \ldots$ positive roots. When $z>1$ the number of positive roots is infinite.

See also I A $A_{2} 34, B_{7} 28$; XII A

\section{Bessel Functions of the Second Kind} AND INTEGRAL ORders $Y_{n}(x), Y^{(n)}(x), G_{n}(x)$

When $n$ is an integer, $J_{n}(x)$ and $J_{-n}(x)$ are not independent solutions of

$$
x^{2} y^{\prime \prime}+x y^{\prime}+\left(x^{2}-n^{2}\right) y=0 .
$$

A solution $Y_{n}(x)$ independent of $J_{n}(x)$ is defined by

$$
\text { (2) } \begin{aligned}
Y_{n}(x)=(1 / \pi) \sum_{r=0}^{\infty}(-1)^{r}\left(\frac{1}{2} x\right)^{n+2 r} H_{r} /[r !(n+r) !] \\
-(1 / \pi) \sum_{r=0}^{n-1}\left(\frac{1}{2} x\right)^{2 r-n} \Gamma(n-r) / r !
\end{aligned}
$$

where $H_{r}=2 \ln \left(\frac{1}{2} x\right)+2 \gamma-\sum_{m=1}^{n+r} m^{-1}-\sum_{m=1}^{r} m^{-1}$, and where $\gamma$ is Euler's constant $.5772156649 \cdots . Y_{n}(x)$ is Bessel's function of the second kind as defined by WEBER ${ }^{1}$ (who gave it, however, in the form of an integral), and is expressible in terms of functions of the first kind by the formula

$$
Y_{n}(x)=\left[J_{n}(x) \cos n \pi-J_{-n}(x)\right] / \sin n \pi
$$

(or the limit of this when $n$ is an integer).

As used by Neumann, ${ }^{2}$ Bessel's function of the second kind is defined by

$$
Y^{(n)}(x)=\frac{1}{2} \pi Y_{n}(x)+(\ln 2-\gamma) J_{n}(x) .
$$

Hankel's function ${ }^{3}$ is $\pi Y_{n}(x)$. The function $G_{n}(x)$ used by GRAY and MATHEWS, $1_{1}-1_{2}, 1895-1922$, is

$$
G_{n}(x)=-\frac{1}{2} \pi Y_{n}(x)=(\ln 2-\gamma) J_{n}(x)-Y^{(n)}(x) .
$$

This is the function tabulated by Arrey 4, 6, 9, 11, 13, and B. A. Smith 2. Nicholson tabulates $2 G_{n}(x)$. In AIREY $1,4,8$, and 11 , the function $Y^{(n)}(x)$ is denoted by $Y_{n}(x)$. Equivalents for our $Y_{n}(x)$ in other writers are the following: $N_{n}(x)$ (AIREy 1, JAhNke \& EMde $1_{1}-1_{5}$ ); $-2 G_{n}(x) / \pi$ (AIrey 4, 6 , 9, 11, 13, Aldis 2, Gray \& Mathews $1_{2}$, Smith 2); $2 K_{n} / \pi$ (Bôcher, McMahoN, Schläfli); $Y_{n}(x) / \pi$ (HANKel); $-G_{n}(x)$ (Heaviside); $-2 K_{n}(x) / \pi$ (HEINE). This display refutes the contention of some recent writers that the notation $N_{n}(x)$ is most generally employed. It was obvious to us that since BAASMTC 1 used $Y_{n}(x)$ we should do the same. 
Auxiliary functions for interpolating $Y_{0}(x)$ and $Y_{1}(x)$, when $x$ is less than .5 , are defined by

$$
Y_{0}(x)=C_{0}(x)+D_{0}(x) \log x, \quad Y_{1}(x)=C_{1}(x) / x+D_{1}(x) \log x .
$$

We noted that Leonard Euler and DANiel Bernoull wrote papers on the vibration of a heavy string suspended from one end in which Bessel functions of the zeroth order occur. In a later paper (presented to the St. Petersburg Academy in 1774, but not published till 1784) treating a similar question, the Bessel function of the second kind, of the zeroth order, is introduced. ${ }^{4}$ p. 148.

${ }^{1}$ H. Weber, J.f. d. reine u. angew. Math., v. 76, 1873, p. 9; Math. Annalen, v. 6, 1873,

${ }^{2}$ C. G. Neumann, Theorie der Bessel'schen Funktionen, Leipzig, 1867, p. 41.

${ }^{8}$ H. HANKeL, Math. Annalen, v. 1, 1869, p. 471.

' L. EULER, "De perturbatione motus chordarum ab earum pondere oriunda," Akad. Nauk, Acta, for 1781, St. Petersburg, 1784, p. 187. Compare M. Bôcher, New York, Math. So., Bull., v. 2, 1893, p. 108-109. In his Institutionum Calculi Integralis, v. 2, St. Petersburg, 1769 , p. 191-192, Euler gave also the complete solution of $x^{3 / 2} y^{\prime \prime}+a y=0$; solutions of this equation are $x^{1} J_{2}\left(4 a^{4} x^{1 / 4}\right), \pi x^{3} Y_{2}\left(4 a^{4} x^{1 / 4}\right)$. See WATson 3 , p. 62 .

$$
\text { A } . Y_{0}(x) \text { and } Y_{1}(x)=-Y_{0}^{\prime}(x)
$$

1. 15D, BAASMTC $6, x=0(.1) 21.5$

2. 14-17D, HAYASHI $3, x=16(1) 25$

3. 10S, BAASMTC $3, x=0(.1) 25$

4. 10D, HAYASHI $3, x=16(.01) 25.51$

5. 10D, NYMTP $6, x=0(.01) 10$

6. 8D, BAASMTC $1, x=0(.01) 25, \delta^{2}$

7. 7D, Watson $3, x=0(.02) 16$, and .1(.1) $5(1) 12$

8. 5D, HAYASHI $2, x=0(.01) 16$

9. 4D, JAHNKE \& EMDE $1_{2}-1_{5}, x=0(.01) 15.99$ (for $x<.8,3 \mathrm{D}$ ); $1_{1}, x=0(.1) 10.2$

10. $4 \mathrm{D}$, GlazenaP, $x=0(.02) 16$

11. $4 \mathrm{D}$, MCLACHLAN $2, x=0(.1) 15.9$

12. $4 \mathrm{D}$, MoRSE, p. $333, x=0(.1) .2(.2) 8$

$$
\text { A. } Y_{n}(x) \text {, including } n \text { (integer) }>1
$$

1. 13-20D, $n=0(1) 21$, BAASMTC $6, x=.1(.1) 25.5, \delta^{2}$

2. $17-13 \mathrm{D}, n=0$ (1) 15 , HAYASHI $3, x=16(1) 25$

3. 15 to $-2 \mathrm{D}, n=16(1) 30$, HAYASH $3, x=16(1) 25$

4. $10 \mathrm{~S}, n=0$ (1)20, BAASMTC $3, x=.1(.1) 25$

5. $8 \mathrm{~S}, n=2(1) 12$, BAASMTC $3, x=0(.1) 25, \delta_{m}^{2}$

6. 7D, $Y_{n}(n), n^{\ddagger} Y_{n}(n), x=n=1(1) 50$, WATSON 3

7. 7D, $Y_{n}(x), n=0(1) 13$, Watson $3, x=6(1) 12$

8. 7-14S, $n=0$ (1) 10, Watson $3, x=.1(.1) 5$

9. $6 \mathrm{D}, n=x, x-1$, AIREY $20, x=0(1) 10$

10. $5 \mathrm{D}, n=2$, TVERITIN, $x=.01(.01) 10$

11. $4 \mathrm{D}, n=2$, MORSE, p. $333, x=0(.1) .2(.2) 8$

12. 4-9S, $Y_{n}(p)$, SEITZ $1, p=.02095, n=[0,1 ; 4 \mathrm{~S}],[2 ; 5-6 \mathrm{~S}],[3 ; 6-9 \mathrm{~S}] ; p=.0000419$, $n=0,[1 ; 4 S],[2 ; 5 S]$

\section{A . Functions involving $Y_{n}(x)$ and derivatives}

1. 14D, $y_{n}=x^{n} Y_{n}(x), n=0$ (1)20, BAASMTC $6, x=0$ (.1)6

2. $8 \mathrm{D}, C_{0}(x), D_{0}(x), C_{1}(x), D_{1}(x)$, BAASMTC $1, x=0(.01) .5, \delta^{2}$

3. 8D, $A_{0}(x), B_{0}(x), A_{1}(x), B_{1}(x)$, BAASMTC $1, x=25(.1) 50(1) 150(10) 1150$, and 1000 $(100) 6000, \delta^{2}$ 
4. 5D, $F_{n}(x), n=1(1) 5$, and graphs, HaLLÉ $1,2, n=1(1) 4, x=0(.1) 1.5,3.5(.1) 4.3,6.7$ (.1)7.3; also $n=1,5, x=2(.1) 2.5,5.2(.1) 5.6$. Graphs, $0<x<14$. If $\mathrm{C}$ is Euler's constant, $F_{1}(x)=J_{0}(x), F_{2}(x)=-x J_{1}(x)$,

$F_{3}(x)=J_{0}(x)[-C-\ln (2 x)+\sin (2 x) \operatorname{Si}(2 x)+\cos (2 x) C i(2 x)]+\pi \sin ^{2} x Y_{0}(x)$,

$F_{4}(x)=-x J_{1}(x)[-C-\ln (2 x)+\sin (2 x) \operatorname{Si}(2 x)+\cos (2 x) C i(2 x)]-\pi x \sin ^{2} x Y_{1}(x)$,

$F_{5}(x)=-J_{0}(x)[C+\ln (2 x)+\sin (2 x) S i(2 x)+\cos (2 x) C i(2 x)]+\pi \cos ^{2} x Y_{0}(x) ;$

$F_{3}(x)+F_{5}(x)=\pi Y_{0}(x)-2 J_{0}(x)[C+\ln (2 x)]$

5. 7D, $\phi(x)=\int_{0}^{\infty}\left[t+\frac{1}{2}-\left(t+t^{2}\right)^{3}\right] \sin x t d t=x / 8+\pi\left[J_{1}\left(\frac{1}{2} x\right) \cos \frac{1}{2} x+Y_{1}\left(\frac{1}{2} x\right) \sin \frac{1}{2} x\right] / 4 x$, Watson $5, x=0(.01) .4(.1) 32 ; x<20.1, \delta^{2}$

6. $3 \mathrm{D}, \phi(x)$ (as in no. 5), Della Moglie, $x=0$ (1)20, and graph $0<x<20$

7. 3-5S, $\pi^{2}\left[J_{n}^{2}(x)+Y_{n}^{2}(x)\right]$, Nicholson 2 , p. 114-115, (a) $n=0(1) 4, x=.1, .4(.1) .6, .8(.1) 1$; (b) $n=0(1) 6, x=1.1,1.2,1.5(.5) 3(1) 6$; (c) $n=7, x=4$ (1)6; (d) $n=8, x=5$, 6; (e) $n=9,10, x=6$

8. $4 \mathrm{D}$ or $4 \mathrm{~S}, \frac{1}{2} \pi\left[.7 J_{1}(x)-x J_{0}(x)\right] Y_{1}(x), \frac{1}{2} \pi\left[.7 Y_{1}(x)-x Y_{0}(x)\right] Y_{1}(x)$, Grammel 2 , $x=0(.1) 10$; graphs, $0<x<10$

9. 8D; 7D, $\log \left[\frac{1}{2} \pi x\left\{J_{n}^{2}(x)+Y_{n}^{2}(x)\right\}\right]^{3} ; \log \left[x\left\{J_{n}^{2}(x)+Y_{n}^{2}(x)\right\}\right]^{3}, n=0(1) 6$, LODGE 4, T. I, II, $x=10(10) 100(100) 1000$

10. 9D; 8D; 8D, $-\sin a_{0} ; \log \left(-\sin a_{0}\right) ; \log \left(-x \sin a_{0}\right)$, LODGE $4, x=10(10) 100(100)-$ 1000. $\left[a_{n}=\frac{1}{2}\left(n+\frac{1}{2}\right) \pi-x+\tan ^{-1}\left\{Y_{n}(x) / J_{n}(x)\right\}\right]$

11. 7D, $\log \left[8 x a_{n} /\left(4 n^{2}-1\right)\right], n=0(1) 6$, LODGE $4, x=10(10) 100(100) 1000$

12. 9-10D, $Q_{n}(x)=\sin a_{n}\left[\frac{1}{2} \pi x\left\{J_{n}^{2}(x)+Y_{n}^{2}(x)\right\}\right]^{3}=\left(\frac{1}{2} \pi x\right)^{3}\left[Y_{n}(x) \cos \left(x-\frac{1}{2} n \pi-\frac{1}{2} \pi\right)\right.$

$-J_{n}(x) \sin \left(x-\frac{1}{2} n \pi-\frac{1}{4} \pi\right)$ ], $n=0(1) 6$, LODGE $5, x=10(10) 100(100) 1000$

13. 9-10D; 8D, $Q_{0}(x) ; \log \left|Q_{0}(x)\right|$, LODGE 5, $x=10(10) 100(100) 1000$

14. 7D, $Y_{n}^{\prime}(n), n^{2 / 3} Y_{n}^{\prime}(n), x=n=1(1) 50$, WATSON 3

15. $(16-p) \mathrm{D}, d^{p} Y_{0}(x) / d x^{p}, p=1(1) 14$, HaYASHI $3, x=16(1) 25$

16. 4-9S, $Y_{n}{ }^{\prime}(p)$, SEITZ $1, p=.02095, n=[0,1 ; 4 \mathrm{~S}],[2 ; 5-6 \mathrm{~S}]$, $[3 ; 6-9 \mathrm{~S}] ; p=.0000419$, $n=0,[1 ; 4 \mathrm{~S}],[2 ; 3 \mathrm{~S}]$

17. 3-4D, $\left[J_{1}^{\prime}(x) J_{1}(x)+Y_{1}^{\prime}(x) Y_{1}(x)\right] / x\left\{\left[J_{1}{ }^{\prime}(x)\right]^{2}+\left[Y_{1}^{\prime}(x)\right]^{2}\right\}$, KALÄHNE $4, x=0(.1)-$ $.5,1,2, \infty$

18. $4 \mathrm{~S},\left\{\left[J_{n}{ }^{\prime}(x)\right]^{2}+\left[Y_{n}{ }^{\prime}(x)\right]^{2}\right\}^{\prime}, \tan ^{-1}\left[J_{n}{ }^{\prime}(x) / Y_{n}{ }^{\prime}(x)\right], n=1(1) 4$, MORSE, p. $338, x=0(.1) \cdot 2(.2) 5$

19. 3-5S, $4\left[\pi^{2}\left\{J_{n}{ }^{\prime}(x)\right\}^{2}+\left\{Y_{n}{ }^{\prime}(x)\right\}^{2}\right]$, Nicholson 2 , p. 123-124,

(a) $n=0(1) 6, x=1.1,1.2,1.5(.5) 3(1) 5$; (b) $n=0(1) 3, x=.5, .6, .8(.1) 1$; (c) $n=7,8$, $x=4$; (d) $n=7(1) 10, x=5$

\section{A. Zeros: $y_{\mathrm{s}}$ of $Y_{0}(x), y_{s}^{\prime}$ of $Y_{1}(x)$}

1. 12D, $y_{s}, y_{s}^{\prime}$, BAASMTC $9, s=1(1) 53, x \ngtr 21.6$

2. $8 \mathrm{D}, y_{s}, Y_{1}\left(y_{s}\right), y_{s}^{\prime}, Y_{0}\left(y_{s}{ }^{\prime}\right)$, BAASMTC $1, s=1(1) 50$

3. $7 \mathrm{D}, y_{s}, y_{s}^{\prime}$, WATSON $3, s=1(1) 40$

4. $5 \mathrm{D}, y_{s}, y_{s}^{\prime}$, HAYASHI 2 , p. $99 s=1(1) 40$

5. $5 \mathrm{D}, y_{s}, y_{o}^{\prime}$, AIREY $1, s=1(1) 10$

6. $4 \mathrm{D}, y_{s}, y_{s}{ }^{\prime}$, GLAZENAP, $s=1(1) 40$

\section{A. Zeros: $y_{n, s}$ of $Y_{n}(x)$, and of functions of $Y_{n}(x)$}

1. 12D, $y_{n, s}, n=0$ (1)20, BAASMTC $9, s=1(1) 53, x \ngtr 21.6$

2. $7 \mathrm{D}, y_{n, s}, n=0(1) 5$, Watson $3, s=1(1) 40$

3.

4. Zeros of $J_{n}(x)+k Y_{n}(x), k=\left(\frac{1}{2} \pi\right) /[\ln 2-\gamma]$, are also zeros of $Y^{(n)}(x)$; see II C,

5. $4 \mathrm{D}, x J_{n}(9 x) Y_{n}{ }^{\prime}(x)-9 x J_{n}^{\prime}(9 x) Y_{n}(x)$, Thilo, p. 554, $n=0,2,3$ (first 4 zeros), $n=1(5$ zeros), $n=4$ ( 3 zeros)

6. 2D, $Y_{0}(x)-E J_{0}(x), E=\ln 2-\gamma$, Colwell \& HARDY, p. 1054, first 10 zeros

7. 2D, $Y_{0}(x)+Y_{1}(x)-E\left[J_{0}(x)+J_{1}(x)\right]$, Colwell \& HaRdy, p. 1053 
8. 2-4D, $J_{n}(x) Y_{n}(k x)-J_{n}(k x) Y_{n}(x), n=0(1) 2$, KALÄHNE 1, and KALÄHNE 2, p. 68-69, and JAHNKE \& EMDE $1_{5} ;$ p. 204-205 (also $1_{2}-1_{4}$ ), first 6 zeros, $x_{n, 8}$, and $(k-1) x_{n, 8}$, $10 k=12,15,20$. Also $1-4 \mathrm{D},(k-1) x_{n, 1}$, p. 81 (J. \& E., p. 205), $k=1,1.2,1.5,2(1)$ $11,19,39, \infty, n=0(1) 2$. Also $3-4 \mathrm{D},(k-1) x_{n, s}, \mathrm{~J} . \&$ E., p. 205, $k$ various, $s=1(1) 4$

9. 3D (mostly), $J_{n}(x) Y_{n}(k x)-J_{n}(k x) Y_{n}(x), k=1.5,2,5,10,20, \infty$, REINSTEIN, $x_{n, s}$ $n=0, s=1(1) 6 ; n=1, s=1(1) 3 ; n=2,3, s=1,2 ; n=4(1) 6, s=1$

10. 6D, $J_{0}(x) Y_{0}(k x)-J_{0}(k x) Y_{0}(x), k=1 \frac{1}{2}\left(\frac{1}{2}\right) 4$, LowaN \& HillmaN, $x_{0, s} s=1$ (1)5. Also $(k-1) x_{0, s}, k=1\left(\frac{1}{2}\right) 4, s=1(1) 5$

11. 2D, $J_{0}(x) Y_{0}(k x)-Y_{0}(x) J_{0}(k x)$, and $J_{1}(x) Y_{0}(k x)-Y_{1}(x) J_{0}(k x), k^{2}=.2(.2) 1$, DiNNIK 10 , p. 23-24, first zeros

12. 2-3D, $J_{0}(x) Y_{1}\left(x e^{l m}\right)-Y_{0}(x) J_{1}\left(x e^{l m}\right)$, DiNNIK 12, zeros, $e^{m}=k^{2}=.01, .2(.2) 1$

13. 2D, $J_{1}(x) Y_{1}\left(x e^{\jmath m}\right)-Y_{1}(x) J_{1}\left(x e^{\jmath m}\right)$, DiNNIK 10 , p. 81, and 12, zeros; see no. 12

14. 4-6D, $J_{1}(x) Y_{1}(k x)-J_{1}(k x) Y_{1}(x), k=1.2,1.5,2,3, s=[1(1) 6 ; 4 \mathrm{D}] ; k=5, s=[1(1) 7$; 5D]; $k=10, s=[1(1) 8 ; 6 \mathrm{D}]$, MUSKAT, MORGAN \& MERES

15. 3D, $J_{1}(x) Y_{1}(k x)-J_{1}(k x) Y_{1}(x)$, OLsson, first zeros, $\alpha=k x$ corresponding to $k=1,1.2$,$1.31,1.45,1.5,1.591,1.831,2,2.528,3(1) 11,19,39, \infty$

16. $2 \mathrm{D} ; 3 \mathrm{D}, Y_{1}(a x) / J_{1}(a x)=\left[h x Y_{1}(b x)+Y_{0}(b x)\right] /\left[h x J_{1}(b x)+J_{0}(b x)\right]$, WEINBERG, first zeros, $h=1, k=a / b=1.02,1.039$ (var.) $2.88 ; 3.93$ (var.) 23.93. Also first eight zeros, $2 S, h=1, k=20$

17. $4 \mathrm{D}, \frac{3 x Y_{0}(x)-2 Y_{1}(x)}{3 x J_{0}(x)-2 J_{1}(x)}-\frac{3 k x Y_{0}(k x)-2 Y_{1}(k x)}{3 k x J_{0}(k x)-2 J_{1}(k x)}, k=\frac{1}{4}, \frac{1}{2}, \frac{3}{4}$, AIREY 5, first 10 zeros

18. $2 \mathrm{D}, \frac{x J_{0}\left(\frac{1}{2} x\right)-4 J_{1}\left(\frac{1}{2} x\right)}{x Y_{0}\left(\frac{1}{2} x\right)-4 Y_{1}\left(\frac{1}{2} x\right)}-\frac{J_{0}\left(\frac{3}{2} x\right)+\left[6 x-\frac{4}{3} x^{-1}\right] J_{1}\left(\frac{3}{2} x\right)}{Y_{0}\left(\frac{3}{2} x\right)+\left[6 x-\frac{4}{3} x^{-1}\right] Y_{1}\left(\frac{3}{2} x\right)}$, GRAMMEL 1 , first two zeros

19. 2D, $\tan \frac{\left(1-k^{2}\right) \lambda x}{4(l-\lambda)}-\frac{J_{1}(k x) Y_{0}(x)-Y_{1}(k x) J_{0}(x)}{J_{1}(k x) Y_{1}(x)-Y_{1}(k x) J_{1}(x)}, \quad k^{2}=0, .1, .2(.2) .8, \quad \lambda / l=0(.2) .8$, DinNik 13, first zeros. There are 4 other similar equations and tables.

20. 3D, $J_{0}(k x) Y_{2}(x)-J_{2}(k x) Y_{0}(x)$, AIREY 3, first 4 zeros, for $k^{2}=1.1,1.2,1.5,2,2.25,3,4,-$ $9,16,25$

\section{A6. Graphs: $Y_{n}(x)$, etc.}

1. $Y_{0}(x),-Y_{1}(x)$, Kalähne 3, and Jahnke \& Emde $1_{1}$, fig. 35, and DahL, $0<x<10$

2. $Y_{0}(x)$, McLaChLAN 2 , p. $9,0<x<8$

3. $-Y_{n}(n),-Y_{n}(n+1)$, JAHNKE \& EMDE $1_{2}$, fig. 133 and $1_{3}-1_{6}$, fig. 100, $0<x(=n)$ $<50$

4. $Y_{n}(x)$, JAHNKe \& EMDE $1_{2}$, figs. 134-135, and $1_{3}-1_{5}$, fig. 101-102, $n=0(1) 13$, $0<x<12$

5. $Y_{n}(x)=$ const in the $n, x$ plane, JAHNKE \& EMDE $1_{2}$, fig. 136, and $1_{3}-1_{5}$, fig. 103, $-5<n<+6,0<x<16$

6. $y_{n, 8}-n$, JAHNKE \& EMDE $1_{2}$, fig. 138, and $1_{3}-1_{5}$, fig. 105, $0<n<5, s=1$ (1) 15

7. $y_{1}=J_{0}(x) / Y_{0}(x), y_{2}=J_{1}(x) / Y_{1}(x), 0<x<12,-5<y_{1}<+5,-5<y_{2}<+5$, J. FISCHER 2, graphs for solving the equation $y_{1}=y_{2}$

8. (a) $x=Y_{0}\left(\frac{1}{2} \pi y\right) / J_{0}\left(\frac{1}{2} \pi y\right)$, (b) $x=Y_{1}\left(\frac{1}{2} \pi y\right) / Y_{0}\left(\frac{1}{2} \pi y\right)$, (c) $x=Y_{1}\left(\frac{1}{2} \pi y\right) / J_{1}\left(\frac{1}{2} \pi y\right)$, JAHNKE \& EMDE $1_{2}$, figs. $140-142,1_{3}-1_{5}$, figs. 107-109, $-1<x<1$ and $-1<(1 / x)<+1$

9. $J_{n}\left(x_{s}\right) Y_{n}\left(k x_{s}\right)-Y_{n}\left(x_{s}\right) J_{n}\left(k x_{s}\right)$, JAHNKE \& EMDE $1_{3}-1_{5}$, fig. 111, graph for $x_{1}=$ $\pi(1+\alpha) /(k-1), .5<n<2.5,0<(1 / k)<1 ; \alpha=.01(.01) .07, .1(.05) .3(.1) .6$. Also graphical solutions, figs. 111-115, of the equation $J_{0}\left(x_{8}\right) Y_{1}\left(k x_{s}\right)-Y_{0}\left(x_{8}\right) J_{1}\left(k x_{s}\right)$, for $x_{s}=(n-.5) \pi(1+\alpha) /(k-1)$

10. $J_{1}{ }^{\prime}(x) / Y_{1}{ }^{\prime}(x)$, SCHELKUNOFF, p. $327,0<x<1.9$

11. $J_{n}{ }^{\prime}(x) / Y_{n}{ }^{\prime}(x), n=1(1) 4,0<x<6$, TRUELL; also graphs of the first zeros of $J_{n}{ }^{\prime}(x) Y_{n}{ }^{\prime}(k x)-J_{n}{ }^{\prime}(k x) Y_{n}{ }^{\prime}(x)$, for $n=1(1) 4,1<k<9$. About $2 \mathrm{D}$ accuracy for the zeros

12. $J_{0}(x) Y_{0}(y)-Y_{0}(x) J_{0}(y)$, and $Y_{1}(x) J_{0}(y)-J_{1}(x) Y_{0}(y)$, SCHELKUNOFF, p. 271, graphs determining the zeros, $0<x<1,0<y<4$ 
13. $F_{5}=-\left[x Y_{0}(x)-.709 Y_{1}(x)\right] /\left[x J_{0}(x)-.709 J_{1}(x)\right]$,

$F_{6}=-\left[.8 x Y_{0}(.8 x)-.709 Y_{1}(.8 x)\right] /\left[.8 x J_{0}(.8 x)-.709 J_{1}(.8 x)\right]$,

FreLd, graphs, $0<x<2.8$, leading to a graphical solution of the equation $F_{5}=F_{6}$ (about 1.07)

See also II $\mathbf{A}_{\mathbf{z}} 8$

$$
\text { B. } G_{0}(x)=-\frac{1}{2} \pi Y_{0}(x) \text { and } G_{1}(x)=-\frac{1}{2} \pi Y_{1}(x)
$$

1. 21D, AlDIS $2, x=.1(.1) 6$

2. $14 \mathrm{D}$, AIREY $17, x=9,10$

3. 10D, AIREY $11, x=6.5(.5) 15.5$

4. 7D, AIREY $6, x=.01(.01) 16$

5. 7D, AIREY $4, x=.1(.1) 16$

6. 5D, AIREY $9, x=.1(.1) 6(.5) 16$

7. $4 \mathrm{D}$, Airey 7, $x=.01(.01) 16$

8. 4D, B. A. Sмith $2, x=0(.01) 1(.1) 10.2$, and $G_{0}(10.3)$

9. 4D, JahNKe \& EMDE 1 , p. 126-128: (a) abridged Aldis $2, x=.1$ (.1)6; (b) B. A. SMith 2. There are 35 cases of contradiction between the corresponding values of these two tables.

10. $4 \mathrm{D}$ or $4 \mathrm{~S}, 2 G_{0}(x), 2 G_{1}(x)$, Nicholson $2, x=.1, .4(.1) .6, .8(.1) 1.2,1.5(.5) 3(1) 6$

$$
\mathrm{B}_{2 .} G_{n}(x)=-\frac{1}{2} \pi Y_{n}(x) \text {, and derivatives }
$$

1. $6 \mathrm{D}, n=x, x-1$, AIREY 13A, $x=1(1) 50(5) 100(10) 200(20) 400(50) 1000(100) 2000(500)$ $5000(1000) 20000(5000) 30000(10000) 50000,100000,500000,1000000$

2. $6 \mathrm{D}, n=x, x-1$, AIREY $13 \mathrm{~B}, x=6(1) 13$

3. $6 \mathrm{D}, n=100(1) 107$, AIREY 13B, $x=104$

4. $5 \mathrm{D}, n=0(1) 13$, AIREY $9, x=.1(.1) 6(.5) 16$; with one exception values of $G_{n}(x)>10$ are omitted.

5. $\leq 4 \mathrm{~S},-2 G_{n}{ }^{\prime}(x)$, Nicholson 2 , p. 122-123, (a) $n=1(1) 3, x=.5(.1) 1$; (b) $n=1$ (1)6, $x=1.2,1.5(.5) 3(1) 5$; (c) $n=7,8, x=4$; (d) $n=7(1) 10, x=5$

6. $3-4 \mathrm{~S}, 2 G_{n}(x)$, Nicholson 2 , p. $113-114$, (a) $n=0(1) 4, x=.1,4(.1) .6,8(.1) 1$; (b) $n=0(1) 6, x=1.1,1.2,1.5(.5) 3(1) 6$; (c) $n=7, x=3(1) 6$; (d) $n=8, x=4$ (1)6; (e) $n=9, x=5,6$; (f) $n=10, x=6$

7. 5D, $\left[\left\{N_{m}(x) / D_{m}(x)\right\}+\frac{1}{2} i \pi\right]^{-1},\left[\left\{N_{m}^{\prime}(x) / D_{m}{ }^{\prime}(x)\right\}+\frac{1}{2} i \pi\right]^{-1}$, RAYLEIGH 9, $m=0(1) 6$, $x=.4(.4) 2.4 .\left[N_{m}(x)=x G_{m}^{\prime}(x) J_{m}\left(x^{\prime}\right)-x^{\prime} G_{m}(x) J_{m}{ }^{\prime}\left(x^{\prime}\right), x^{\prime}=1.5 x\right.$,

$D_{m}(x)=x^{\prime} J_{m}(x) J_{m-1}\left(x^{\prime}\right)-x J_{m}\left(x^{\prime}\right) J_{m-1}(x)$,

$N_{m}^{\prime}(x)=x^{\prime} J_{m}\left(x^{\prime}\right) G_{m}^{\prime}(x)-x J_{m}^{\prime}\left(x^{\prime}\right) G_{m}(x)$,

$\left.D_{m^{\prime}}^{\prime}(x)=x J_{m}(x) J_{m-1}\left(x^{\prime}\right)-x^{\prime} J_{m}\left(x^{\prime}\right) J_{m-1}(x)+m\left(\frac{x^{\prime}}{x}-\frac{x}{x^{\prime}}\right) J_{m}(x) J_{m}\left(x^{\prime}\right)\right]$

$C_{1 \cdot} Y^{(0)}(x)$ and $Y^{(1)}(x)$

1. $14 \mathrm{D}$, AIREY $17, x=9,10$

2. 10D, AIREY $11, x=.1(.1) 6(.5) 15.5$

3. 7D, AIREY $4, x=.1(.1) 16$

4. $6 \mathrm{D}$, AIREy $8, x=.02(.02) 15.5$

5. $6 \mathrm{D}$, AIREy $12, x=.2(.2) 6(.5) 15.5$

6. 4 D, B. A. SмITH $1, x=0(.01) 1(.1) 10.2$

$\mathrm{C}_{2} . Y^{(n)}(x)$, including $n>1$

1. $6 \mathrm{D}, n=0(1) 13$, AIREY 12, $x=.2(.2) 6(.5) 15.5$; most values of $Y^{n}(x)>10$ are omitted

2. $6 \mathrm{D}, n=x, x-1$, AIREY $13 \mathrm{~A}, x=1(1) 50(5) 100$ etc., as in II $\mathrm{B}_{2} 1$

$$
\text { C. } \boldsymbol{Y}^{(n)}(x) \text {, zeros }
$$

1. $6 \mathrm{~S}, Y^{(100)}(x)$, AIREY 14 , first 5 zeros

2. 5D, $Y^{(0)}(x), Y^{(1)}(x), Y^{(2)}(x)$, AIREY 1, p. 221-222, first 40 zeros 
3. 3D, $Y^{(n)}(x)$, KALÄHNE $1, n=0$ (first 4 zeros), $n=1$ (first 3 zeros)

4. $4 \mathrm{D}, y_{s+w} / y_{1}, y_{v+w}-y_{1},\left(y_{s}\right.$ being zeros of $\left.Y^{(n)}(x)\right)$, KALÄHNE $1, n=0, s=1, w=1(1) 3$; $s=2, w=1,2$, and $s=3, w=1 ; n=1, s=1, w=1,2$, and $s=2, w=1$

5. 5D, $J_{0}(x)+Y^{(0)}(x), \quad J_{1}(x)+Y^{(1)}(x), \quad J_{0}(x)-Y^{(0)}(x), \quad J_{0}(x)-2 Y^{(0)}(x), \quad 10 J_{0}(x)$ $+Y^{(0)}(x), 10 J_{0}(x)-Y^{(0)}(x)$, AIREY 1, p. 224, first 10 zeros

6. 3D or 3S, $Y^{(0)}(x)+k J_{0}(x)$, KALÄHNE 2 , p. $72, k=0(1) 3$, first 2 zeros (except $k=$ 0,4 zeros); values found graphically

7. 3D or 3S, $Y^{(1)}(x)+k J_{1}(x)$, KALÄHNE 2 , p. $72, k=0(1) 4,10$, 20, first 2 zeros (except $k=0,3$ zeros); values found graphically

III. Modified Bessel Functions of the First and Second KIND- $I_{n}(x), K_{n}(x)$. Bessel Functions OF THE THIRD KIND- $H_{n}{ }^{(1)}(x), H_{n}{ }^{(2)}(x)$

If in Bessel's equation [I (1) and II (1)] the signs of the third term and before $n^{2}$ are changed we get

$$
x^{2} y^{\prime \prime}+x y^{\prime}-\left(x^{2}+n^{2}\right) y=0
$$

which has independent solutions $I_{n}(x)$ and $I_{-n}(x)$, where

$$
I_{n}(x)=\sum_{s=0}^{\infty}\left(\frac{1}{2} x\right)^{n+2 s} /[s ! \Gamma(n+s+1)],
$$

except when $n$ is an integer. $I_{n}(x)$ is the modified Bessel function of the first kind.

If $n$ is an integer

$$
I_{-n}(x)=I_{n}(x)=\sum_{s=0}^{\infty}\left(\frac{1}{2} x\right)^{n+2 s} /[s !(n+s) !]
$$

For $2 n>-1$

(4) $I_{n}(x)=(i)^{-n} J_{n}(i x)=\left(\frac{1}{2} x\right)^{n} /\left[\Gamma\left(n+\frac{1}{2}\right) \pi^{\frac{1}{2}}\right] \int_{0}^{\pi} \cosh (x \cos \phi) \sin ^{2 n} \phi d \phi$.

The modern notation $I_{n}(x)$ was introduced by A. B. BASSET, ${ }^{1}$ possibly, because of important work in connection with the function by JAMES IVORY. ${ }^{2}$

When $n$ is an irsteger a second solution of equation (1) is $K_{n}(x)$, defined by

$$
\begin{aligned}
K_{n}(x)= & (-1)^{n+1}\left\{\gamma+\ln \left(\frac{1}{2} x\right)\right\} I_{n}(x) \\
& +\frac{1}{2} \sum_{s=0}^{n-1}(-1)^{s}(n-s-1) !\left(\frac{1}{2} x\right)^{-n+2 s} / s ! \\
& +(-1)^{n \frac{1}{2}} \sum_{s=0}^{\infty}\left(\frac{1}{2} x\right)^{n+2 s} /[s !(n+s) !] \\
& \times\left\{\frac{1}{1}+\frac{1}{2}+\frac{1}{3}+\cdots+\frac{1}{s}+\frac{1}{1}+\frac{1}{2}+\cdots+\frac{1}{n+s}\right\},
\end{aligned}
$$

where $\gamma$ is Euler's constant $.5772156649 \ldots . K_{n}(x)$ is the modified Bessel function of the second kind.

Basset's use of the notation ${ }^{1} K_{n}(x)$ was possibly because of the discussion of the functions by KUMMER. ${ }^{3}$ 
The series for $I_{0}(x), I_{1}(x), K_{0}(x), K_{1}(x)$ may be readily written down. When $x$ is small $(<.5)$ interpolation for $K_{0}(x)$ and $K_{1}(x)$ may be carried through with the aid of tabulated auxiliary functions $E_{0}(x), E_{1}(x), F_{0}(x)$, $F_{1}(x)$, since

$$
K_{0}(x)=E_{0}(x)+F_{0}(x) \log x, \quad K_{1}(x)=E_{1}(x) / x+F_{1}(x) \log x .
$$

The Bessel functions of the third kind $H_{n}(x)$ are defined by the relations

$$
H_{n}{ }^{(1)}(x)=J_{n}(x)+i Y_{n}(x), \quad H_{n}{ }^{(2)}(x)=J_{n}(x)-i Y_{n}(x) .
$$

The symbol $H$ was used by NiELSEN who employed the term Cylindrical functions of Hankel. ${ }^{4}$

Tables have been computed for $e^{-x} I_{n}(x)=\left(2 / \pi^{\frac{1}{3}}\right) \int_{0}^{\infty} e^{-u^{2}} J_{2 n}\left[2 u(2 x)^{\frac{1}{2}}\right] d u$, and $e^{x} K_{n}(x)=\left(4 / \pi^{\frac{1}{3}}\right) \cos (n \pi) \int_{0}^{\infty} e^{-u^{2}} K_{2 n}\left[2 u(2 x)^{\frac{1}{3}}\right] d u, \quad n=0, \quad 1 ; \quad$ for $\Phi_{n}(x)=(2 \pi x)^{\frac{1}{2}} e^{-x} I_{n}(x)=w_{n}(x)-e^{-2 x} w_{-n}(-x) ;$ and for $i_{n}(x)=x^{-n} I_{n}(x)$, $k_{n}(x)=x^{n} K_{n}(x)$.

ANDING uses $L_{n}(x)$ for our $I_{n}(x)$; and LoDGE employs $R$ for $(\pi x / 2)^{1}\left|H_{n}{ }^{(1)}(x)\right| . I_{n}(x)$ may be expressed in terms of a confluent hypergeometric function as follows

$$
\left(\frac{1}{2} x\right)^{n} e^{x}\{1 / \Gamma(n+1)\} F\left(n+\frac{1}{2} ; 2 n+1 ;-2 x\right) .
$$

For our $I_{n}(x)$, JAHNKE \& EMDE $1_{1}$ used $i^{-n} J_{n}(i x)$ and for our $K_{0}(x)$ and $K_{1}(x)$ used $(i \pi / 2) H_{0}{ }^{(1)}(i x)$ and $-(\pi / 2) H_{1}{ }^{(1)}(i x)$ respectively.

It may be well to explain the notation of the following original form of Kirchhoff's Equation, (arising in the study of the vibration of a free uniform circular plate) which occurs as equation (15), p. 74, of KIRCHHOFF 1 :

$$
\begin{gathered}
8 \gamma n^{2} z^{2} X^{(n)} Y^{(n)}-8 \gamma n^{2} z^{3}\left[X^{(n)} \frac{d Y^{(n)}}{d z}+Y^{(n)} \frac{d X^{(n)}}{d z}\right] \\
-\left[n^{2}\left(n^{2}-1\right) z+16 \gamma^{2} z^{5}\right]\left[X^{(n)} \frac{d Y^{(n)}}{d z}-Y^{(n)} \frac{d X^{(n)}}{d z}\right]+8 \gamma z^{4} \frac{d X^{(n)}}{d z} \frac{d Y^{(n)}}{d z}=0 ; \\
X^{(n)} \equiv I_{n}(2 z), \quad Y^{(n)} \equiv J_{n}(2 z), \quad \gamma^{-1}=1-\sigma, \quad 2 z=x .
\end{gathered}
$$

These substitutions having been made, the form of the equation given in division $A_{5}$ of this Section may be derived. The roots of the equation are $x=2$ " $\lambda_{n} \mu l, " \mu=0(1) 2$ for each $n=0(1) 3$.

There is a discussion of this paper, KIRChHOFF 1, as well as of an intimately related paper, KIrchhoff 2, in I. Todhunter, $A$ History of the Theory of Elasticity . . ., v. 2, pt. 2, Cambridge, 1893, p. 39-50.

1 A. B. BAsser, A Treatise on Hydrodynamics, with numerous Examples, 2v, Cambridge, 1881 , v. 2 , p. 15 .

${ }^{2}$ J. IvoRy, R. So. London, Trans., v. 113, 1823, p. 409, 495; and v. 128, 1838, part 2, p. $170-229$.

${ }^{3}$ E. E. Kummer, J.f.d. reine u. angew. Math., v. 12, 1834, p. 144-147; and v. 17, 1837, p. 210-242.

4 N. Nielsen, Handbuch der Theorie der Cylinderfunktionen, Leipzig, 1904, p. 16. 


$$
\text { A. } I_{0}(x)=J_{0}(i x), \text { and } I_{1}(x)=I_{0}^{\prime}(x)=-i J_{1}(i x)
$$

1. $21 \mathrm{D}$, Aldis $1, x=.1(.1) 6$

2. $18 \mathrm{D}$, ALDIS $1, x=6(1) 11$

3. $15-18 \mathrm{D}, \mathrm{BAASMTC} 6, x=0(.1) 20$

4. 12D, LodGe 1 , and Gray \& Mathews $1_{1}-1_{2}, x=0(.2) 6, \Delta$

5. 10D, NYMTP 3, p. 362-381, $x=0(.01) 10$; also NYMTP 6 , for the same range

6. 10S, BAASMTC $4, x=0(.1) 20$

7. $9 \mathrm{D}$, LODGE $3,2, x=0(.001) 5.1, \Delta$

8. 9D, Gray \& Mathews $1_{2}, x=0(.01) 1$

9. $8 \mathrm{D} ; 7 \mathrm{D}$, BAASMTC $1, x=0(.001) 4 ; 4(.001) 5, \delta^{2}$

10. $6 \mathrm{~S}$, WRINCH \& WRINCH $1,2, x=5(1) 37$

11. 5S, MoRse, p. $334, x=0(.1) \cdot 2(.2) 8$

12. $5 \mathrm{D}$, Dwight $5, x=0(.1) 6$

13. $4 \mathrm{D}, \mathrm{DALE}$, and IsHeRWOOD (LODGE 1 abridgements) $x=0(.2) 5$

14. 4-6S, OlLENDORFF $2, x=0(1) 15$

15. 4-5S, JAHNKE \& EMDE $1_{2}$ (p. 278f) - $1_{5}$ (p. 226f), $x=0(.01) 9.99, \Delta$, and $0(.2) 6$

16. $6 \mathrm{~S} ; 4-5 \mathrm{D}$, JAHNKE \& EMDE $1_{1}, I_{0}(x)$ (AlDIS 1 abridgement), p. $130, x=0(.1) 6(1) 11$; $I_{1}(x)$ (LODGE 3 abridgement), p. 131-133, $x=0(.01) 5.1(.1) 6(1) 11$

17. 4 D, DiNNIK 11 , p. $122,125-126, x=0(.2) 8$

A2. $I_{0}(x)$ or $I_{1}(x)$; functions involving $I_{0}(x)$ and (or) $I_{1}(x)$

1. $15 \mathrm{D}, I_{0}(x)$, NYMTP $1, x=0(1) 10$

2. $4 \mathrm{D}, I_{0}(x)$, BYERLY $2, x=0(.1) 5.9$

3. $4 \mathrm{~S}, I_{0}(x)$, FuRNAS, $x=12(2) 20(10) 100(100) 1000$

4. 3-4S, $I_{0}(x)$, Colwell \& HaRdy, p. $1046, x=0,1,5,10,20,30$

4A. 20S, $I_{0}(30)$, WRENCH

5. $15 \mathrm{D}, I_{0}(x), I_{1}(x) / x$, BAASMTC $6, x=0(.1) 6$

6. $4 \mathrm{D}, I_{0}(x), x I_{1}(x)$, RAYLEIGH $5, x=0(.1) .9$

7. 9D, $I_{1}(x)$, Gray \& Mathews $1_{1}, x=0(.01) 5.1$

8. $6 \mathrm{D} ; 6-7 \mathrm{~S}, I_{1}\left(2 x^{3}\right) / x^{3}$, WARD, $x=0(1) 5 ; 3.61,3.6481,6(1) 12,15$

9. $4 \mathrm{D}, I_{1}(x) / I_{0}(x),\left[I_{1}(x) / I_{0}(x)\right]^{3}$, SCHEUERMANN, $x=0(1) 11,3.6(1) 5.6$

10. $4 \mathrm{D},\left[x\left(1-x^{2}\right) I_{1}(x) / I_{0}(x)\right]^{3}$, RAYLEIGH $2, x^{2}=.05, .1(.1) .9$

11. $4 \mathrm{D},\left[x\left(1-x^{2}\right) I_{1}(x) / I_{0}(x)\right]$, RAYLEIGH $5, x=0(.1) 1$

12. $3-4 \mathrm{D}, 1+x^{2}-\left[x I_{0}(x) / I_{1}(x)\right]^{2}$, RAYLEIGH $4, x=0(.2) 1,2(2) 6$

13. 4-5D, $x\left[I_{0}(x) / I_{1}(x)+J_{0}(x) / J_{1}(x)\right]$, PREsCotT, p. 587, $x=0,2,2.98,2.982,3.832$, $6.18,6.2$

14. $3 \mathrm{D}, y=1-.615\left[r_{2} /\left(r_{1}+r_{2}\right)\right] e^{3 r_{1}} S\left(\frac{1}{2} r_{2}\right) / S\left[\frac{1}{2}\left(r_{1}+r_{2}\right)\right]$, where $S(x)=I_{0}(x)+I_{1}(x)$, KoHN,

(a), T. $1: r_{1}=r_{2}=r=.2(.2) .8,2(2) 6,10,20 ;$ graph, $0<r<30$

(b), T. $2: r_{1}=2 r_{2}=.4, .8,1.6,4,8,16,32,44 ;$ graph, $0<r_{1}<60$

(c), T. 3: $r_{1}=10 r_{2}=2.4,8,11,20,40,120,220 ;$ graph, $0<r_{1}<300$

15. $6 \mathrm{D}, \log I_{0}(x), \log \left[I_{1}(x) / x\right]$, ANDING, $x=0(.01) 10$

16. $6 \mathrm{D}, \log \left[x^{3} I_{n}(x)\right], n=0,1$, ANDING, $x=10(.1) 50(1) 200$ (10) 1000 (var.) $10^{6}, \Delta$

17. $5 \mathrm{D}$, mantissas of $\log \left[(2 \pi x)^{4} e^{-x} I_{1}(x)\right]$, HoRT, $x=11(.1) 20.9,21(1) 109$

18. $9 \mathrm{D}, e^{-x} I_{0}(x), e^{-x} I_{1}(x)$, BADELLINO, $x=20$ (1) 50

19. $8 \mathrm{D}, e^{-x} I_{0}(x), e^{-x} I_{1}(x)$, BAASMTC $1, x=5(.01) 10(.1) 20, \delta^{2}$

20. $7 \mathrm{D}, e^{-x} I_{0}(x), e^{-x} I_{1}(x)$, WATSON $3, x=0(.02) 16$

21. 3D; $2 \mathrm{D}, e^{-x} I_{0}(x)$, HeSSELBERG \& BJÖRKDAL, $x=0(.2) 4(1) 11 ; 15(5) 50,100$

22. $4 \mathrm{D}, e^{-x}\left[I_{0}(x)+I_{1}(x)\right]$, WESTGREN, $x=0(.2) 12(.4) 20(1) 36(2) 50$

23. $2 \mathrm{D}, e^{-x}\left[I_{0}(x)+I_{1}(x)\right]$, BuchWALD, $x=1.14,1.36,1.6,1.62,1.92,2.28,2.82,4.3,6.88$, 16.14 , and graph

24. 3D, $x e^{-x}\left[I_{0}(x)+I_{1}(x)\right]$, ELSASSER, $x=.01(.01) 2(.5) 5(1) 10$

25. 3-4S, $x e^{-x}\left[I_{0}(x)+I_{1}(x)\right]$, LADENBURG \& REICHE, $x=.476,2,4(4) 12,20(10) 50,70,200$,$400,500,720,980,1280,2000,2880,4500,6260,7800$. Graphs 
26. $3 \mathrm{D}, e^{-x}\left[(1+2 x) I_{0}(x)+2 x I_{1}(x)\right]$, SMART, p. $43,(2 x)^{3}=0(.1) 2$

27. $2 \mathrm{D}, \frac{1}{2} \pi^{\frac{3}{3}} e^{-x}\left[(1+2 x) I_{0}(x)+2 x I_{1}(x)\right]$, KAPTEYN $\&$ RHIJN, $2 x=0(1) 10(5) 20(10) 100,150$

28. 7D, $(2 \pi x)^{3} e^{-x} I_{n}(x), n=0,1$, OKAYA $2, x=0(.001) .22, \Delta(n=0),-\Delta(n=1)$

29. $7 \mathrm{D},(2 \pi x)^{3} e^{-x} I_{n}(x), n=0,1$, OKAYA $1, x^{-1}=0(.01) .1$

30. $6 \mathrm{D},(2 \pi x)^{3} e^{-x} I_{n}(x), n=0,1$, ANDING, $x=10$ (.1) $50(1) 200(10) 1000$ (var.) $10^{6}, \Delta$

31. $4 \mathrm{D},\left(\frac{1}{2} \pi x\right)^{3} e^{-x}\left[I_{0}(x)+I_{1}(x)\right]$, Eddington, p. 592, and SMarT, p. 127, $(2 x)^{\frac{1}{3}}=0(.1) 2$

32. 1D, $2 k x e^{-k\left(x^{2}+x_{0}^{2}\right)} I_{0}\left(2 k x_{0} x\right)$, ERTEL \& JAW, $k=.1294, x_{0}=6.93, x=2(1) 13 ; k=.0738$, $x_{0}=10.04, x=3(1) 18 ; k=.0448, x_{0}=11.87, x=4(1) 22$

33. 6S, $M\left(\frac{1}{2} .1 . x\right)=e^{\frac{3}{x}} I_{0}\left(\frac{1}{2} x\right), M\left(\frac{3}{2} .3 . x\right)=(4 / x) e^{3 x} I_{1}\left(\frac{1}{2} x\right)$, AIREY 27, p. 231, $x=0(.02) .1(.05) 1(.1) 2(.2) 3(.5) 8$

34. $4 \mathrm{~S}, M\left(\frac{1}{2} .1 . x\right), M\left(\frac{3}{2} .1 . x\right)$, WeBb \& AIREY, $x=1(1) 6(2) 10$

\section{A.$I_{n}(x)$ including $n$ (integer) $>1$}

1. $15-18 \mathrm{D}, n=0(1) 21$, BAASMTC $6, x=0(.1) 20$

2. $12 \mathrm{~S} ; 10-12 \mathrm{~S}, n=0(1) 10 ; 11$, LODGE $1, x=0(.2) 6$

2A. 10S, TAKAGI, $x=1(1) 6, n=0(1) 11$ as in LodGE 1 ; and $x=7(1) 10(10) 100,23<n$ $\leq 54$

3. $10 \mathrm{D}, n=100$, LEHMER $1, x=75$

4. $10 \mathrm{~S}, n=0(1) 20$, BAASMTC $4, x=0(.1) 20$

5. $8 \mathrm{D}, n=2(1) 12$, BAASMTC $4, x=0(.01) 10$

6. $6 \mathrm{~S}, n=0(1) 6$, WRINCH \& WRINCH $1,2, x=5(1) 37$

7. $5 \mathrm{~S}, n=0(1) 11$, Dwight $5, x=0(.1) 6$

8. 5S, $n=0(1) 11$, DALE, $x=0(.2) 5$

9. 5S, $n=0(1) 10$, ISHERWOOD, $x=0(.2) 5$

10. $5 \mathrm{~S}, n=0(1) 2$, MORSE, p. $334, x=0(.1) .2(.2) 8$

11. $4-6 \mathrm{~S}, n=0(1) 9$, OlLENDORFF $2, x=0(1) 15$

12. $4-6 \mathrm{~S}, n=0(1) 11$, JAHNKE $\&$ EMDE $1_{1}$ (p. 159) - $1_{5}$ (p. 232), $x=0(.2) 6$

13. $4 \mathrm{D}, n=0(1) 4$, RODER, $x=0(.1) 1(.2) 2.8,5.6$

See also III A,

\section{A4. Functions of $I_{n}(x)$}

1. $15 \mathrm{D}, i_{n}(x)=x^{-n} I_{n}(x), n=0(1) 22$, BAASMTC $6, x=0(.1) 6$

2. 7D, $e^{-x} I_{n}(x), n=2(1) 5$, WATSON $3, x=.1(.1) 5$

3. $8 \mathrm{~S}, i_{n}(x), e^{-x} I_{n}(x), n=2(1) 20$, BAASMTC $4, x=0(.1) 20$

4. 8D, $i_{n}(x), e^{-x} I_{n}(x), n=2(1) 12$, BAASMTC $4, x=0(.01) 10$

5. $6 \mathrm{D}, J_{n}(x) / I_{n}(x), n=2,3$, WRINCH \& WRINCH $2, x=1(1) 15$

6. $15 \mathrm{D}, \log I_{n}(x), n=20,21$, BAASMTC $6, x=6(.1) 20$

7. $15 \mathrm{D}, \log i_{n}(x), n=21,22$, BAASMTC $6, x=6(.1) 20$

8. $4-5 \mathrm{D}, \log I_{1}(m x)$, MicheLL $2, m=1(2) 11, x / \pi=1(.1) 2$

9. $5 \mathrm{D}, \log \left[e^{-x} I_{n}(x)\right], n=1(1) 6(2) 10$, WAGNER, $x=0(.2) 6,10(10) 40$

10. $4 \mathrm{D}, J_{n}(x i)$ and its derivatives, with suitable factors, $n=0(1) 4$, RIBIÈRE, $8 x / \pi=1(1) 9$

\section{A . Zeros: Kirchhoff's Equation:}

$$
\frac{x^{2} J_{n}(x)+(1-\sigma)\left[x J_{n}^{\prime}(x)-n^{2} J_{n}(x)\right]}{x^{2} I_{n}(x)-(1-\sigma)\left[x I_{n}^{\prime}(x)-n^{2} I_{n}(x)\right]}=\frac{x^{3} J_{n}^{\prime}(x)+(1-\sigma) n^{2}\left[x J_{n}^{\prime}(x)-J_{n}(x)\right]}{x^{3} I_{n}^{\prime}(x)-(1-\sigma) n^{2}\left[x I_{n}^{\prime}(x)-I_{n}(x)\right]}
$$

1. 5D, KIRchнofF $1, \sigma=\frac{1}{4}, \frac{1}{3}, 2$ zeros, $\log \left(\frac{1}{2} x\right)^{4}$, for each $\sigma$, and each $n=0(1) 3$

2. 5S, PRESCOTr, p. 597 (KIRCHHOFF 1), $\sigma=\frac{1}{4}, \frac{1}{3}, 2$ zeros, $x^{4}$, for each $\sigma$, and each $n=0$ (1)3

3. $4 \mathrm{D}$, Krrchioff $2, \sigma=\frac{1}{4}$, zeros, $x^{2}$, are given, 3 each for $n=0,2,3$, and 2 each for $n=1,4,5$; for $\sigma=\frac{1}{3}, 2$ zeros each, to $4 \mathrm{D}$, are given for $n=0(1) 3$

4. 2D, Colwell \& HaRdy, p. 1044, $\sigma=\frac{1}{3}, n=0,1$, first 10 zeros; $n=2(1) 6$, first 11 zeros

5. 3D, OKaya \& TobiIsI, $\sigma=\frac{1}{3}, n=0,1$, first 10 zeros; $n=2(1) 10$, first 11 zeros; $n=11$ first 2 zeros; and $n=12$, first zero 
6. 3D, Airey 2, p. 231-232, (a) $\sigma=\frac{1}{4}, n=0,1$ first 9 zeros; $n=2,3$, first 10 zeros. (b) $\sigma=\frac{1}{3}, n=0,1$, first 4 zeros; $n=2,3$, first 5 zeros. When $n=0$ or 1 , Kirchhoff's equation becomes

$$
\frac{J_{n}(x)}{J_{n+1}(x)}+\frac{I_{n}(x)}{I_{n+1}(x)}=\frac{2(1-\sigma)}{x}
$$

7. A table of solutions of a generalization of Kirchhoff's equation was given by MaLkin. When $\sigma=1$, Kirchhoff's equation reduces to the forms in $A_{8}$.

A6. Zeros: $\begin{aligned} J_{n}(x) I_{n+1}(x)+J_{n+1}(x) I_{n}(x)=J_{n-1}(x) I_{n}(x)-J_{n}(x) I_{n-1}(x) & =-J_{n}(x) I_{n}{ }^{\prime}(x)+J_{n}{ }^{\prime}(x) I_{n}(x)\end{aligned}$

1. 3-5S, Franke, $n=0$ (5 zeros), $n=1,2$ (4 zeros), $n=3(3), n=4(2), n=5,6,7(1)$

2. 5S, Carrington, $n=0$ ( 5 zeros), $n=1,2$ ( 4 zeros), $n=3$ (3)

3. $4 \mathrm{D} ; 3 \mathrm{D}$, Airey $2, n=0 ; 1(1) 3$, first 10 zeros

4. $3 \mathrm{D} ; 4 \mathrm{D}, n=2, x ; x / \pi$, WRINCH, first 10 zeros, in each case

5. 3-4S, Schulze, $n=0$ (5 zeros), 1 (3 zeros), 2(2)

6. 4S, PrescotT, p. 597, $n=0$ (fourth power of 3 zeros), $n=1$ (1 zero), $n=2$ (1)

7. $4 \mathrm{~S}, \mathrm{MoRSE}, \mathrm{p} .175, n=0$ (3 zeros), $n=1(3), 2(3)$

\section{A . Graphs involving $I_{n}(x)$}

1. $I_{0}(x)$, MCLAChlan 2, p. $104,0<x<6$

2. $I_{n}(x), n=0(1) 15$, Colwell, Stewart \& ARnett, $1<x<6$

3. $I_{n}(x), n=0(1) 11$, JAHNKe \& EMDE $1_{2}$ (figs. 144-145) - $1_{5}$ (figs. 118-119)

4. $I_{n}(x), n=0(1) 9$, OLLENDORFF 2

5. Curve with abscissa $y=(x / 3.26)\left[I_{0}(x)+I_{1}(x)\right]$, ordinate $k=2-2\left[1-e^{-x}\left\{I_{0}(2 x)+I_{1}(2 x)\right\} /\left\{I_{0}(x)+I_{1}(x)\right\}\right]$, BoRN, p. 492

6. $I_{n}(x) / I_{n}{ }^{\prime}(x), 0<n<7$, Colwell \& HARDY, p. 1044, $x=2(1) 4,6,10(10) 30$

7. $J_{2}(x) / I_{2}(x), J_{3}(x) / I_{3}(x)$, WRINCH \& WRINCH $2,4 \frac{1}{2}<x<12$

8. $I_{0}(x)\left[20(2 x)^{i}\right] e^{-200-x}$, FURNAS, $125<x<275$

9. $I_{1}(x) /\left[x I_{0}(x)\right]$, RUEDY $1,0<x<4$

10. $\pi x e^{-x}\left[I_{0}(x)+I_{1}(x)\right]$, LadenbuRg \& Reiche, $0<x<3100$; See III $A_{2} 25$

See also III $A_{2} 14,23$

$$
\text { B. } K_{0}(x) \text { and } K_{1}(x)=-K_{0}^{\prime}(x)
$$

1. 21D, ALDIS $1, x=.1(.1) 6$

2. $18 \mathrm{D}$, ALDIS $1, x=6(1) 12$

3. 9-16D, AlDIS $1, x=5(.1) 11.9$

4. 9-21D, Gray \& Mathews $1_{2}$, as in nos. 1-3 above

5. 12-13S, BAASMTC $6, x=.1(.1) 20.5$

6. 8-9S, BAASMTC $1, x=0(.01) 5, \delta_{m}^{2}$

7. 8S, NYMTP $8, x=0(.01) 5, \delta^{2}$

8. 7S, NYMTP $8, x=0(.0001) .03(.001) 1, \Delta^{2}$

9. 7D, WATSON $3, x=0(.1) 5$

10. $5 \mathrm{~S}$, ISHERWOOD, $0(.2) 5$

11. 4 S, JAHNKe \& EMde 11, p. $135-136, x=0(.1) 12$

B. $K_{0}(x)$ or $K_{1}(x)$; functions involving $K_{0}(x)$ and (or) $K_{1}(x)$; zeros

1. $12-15 \mathrm{~S}, K_{0}(x), x K_{1}(x)$, BAASMTC $6, x=.1(.1) 6$

2. $4 \mathrm{D},-K_{0}(x),-x K_{1}(x)$, RAYLEIGH $5, x=0(.1) .9$

3. $10 \mathrm{D},(2 / \pi) K_{0}(x),(2 / \pi) K_{1}(x)$, NYMTP $6, x=0(.01) 10$

4. 8D, $E_{0}(x), F_{0}(x), E_{1}(x), F_{1}(x)$, BAASMTC 1 , p. $265, x=0(.01) .5, \delta^{2}$

5. 7S, $E_{0}(x), F_{0}(x), E_{1}(x), F_{1}(x)$, NYMTP $8, x=0(.001) .03, \Delta^{2}$

6. $5 \mathrm{D}, x K_{0}(x), x K_{1}(x)$, ChandRasekhaR, $x=0(.1) 4.5$; also, to $4 \mathrm{D},(2 / \pi) x K_{1}(x), x=0(.5) 4.5$ 
7. $6 \mathrm{D}, T_{n}(x)=\left(\frac{1}{2} x\right)^{n} K_{n}(x) /\left[\pi^{3}\left(n-\frac{1}{2}\right) !\right], n=0,1$, ELDERTON, $x=0(.1) 4(.5) 16(2) 40(5) 120$

8. 8D, $e^{x} K_{0}(x), e^{x} K_{1}(x)$, BAASMTC $1, x=5(.01) 10(.1) 20, \delta^{2}$

9. 7D, $e^{x} K_{0}(x), e^{x} K_{1}(x)$, WATSON $3, x=0(.02) 16$

10. $4 \mathrm{D}, e^{x} K_{0}(x), e^{x} K_{1}(x)$, KING, $x=.01(.01) .1,5(1) 10 ; x=.02(.01) .1(.1) 3(.2) 6(.5) 10(1) 20$

11. 7D; 6D, log $K_{0}(x)$, Topping \& LudLAM, $x=2(.001) 4 ; 4(.001) 12$

12. $6 \mathrm{~S}-5 \mathrm{~S}, \log \left[K_{1}(m x)\right]$, Michell $2, m=1(2) 11, x / \pi=1(.1) 2$

13. $5 \mathrm{D}, \rho=K_{0}(x) / K_{1}(x)$, Elderton, $x=0(.1) 4(.5) 16(2) 40(5) 120$

14. $4 \mathrm{D},\left[x\left(1-x^{2}\right) K_{1}(x) / K_{0}(x)\right]^{3}$, RAYLEIGH $5, x=0(.1) .9$

15. 5D, $2\left(1+x^{2}\right)^{3} I_{1}(x) K_{1}(x)$, Yost, WhEELER \& BREIT $2, x=.02, .1, .3(.3) .9,1(1) 10(3) 16$

16. $3 \mathrm{D}, J_{1}(x) /\left[x J_{0}(x)\right]+n_{10}{ }^{-2}\left\{K_{1}(y) /\left[y K_{0}(y)\right]\right\}$, BuchHolz 2 ; in the first table for $y=0,06, .1(.1) \cdot 6(.2) 1(.5) 2$, and $n_{10}=9, A=-81 y K_{0}(y) / K_{1}(y)$ is given. Then follow tables of the first two zeros $x$ for each of the 12 values of $y$.

\section{B. $K_{n}(x)$, including $n$ (integer) $>1$; functions of $K_{n}(x)$; graphs}

1. $12-13 \mathrm{~S}, K_{n}(x), n=0(1) 20$, BAASMTC $6, x=.1(.1) 20.5$

2. $10 \mathrm{~S}, K_{n}(x), n=0(1) 20$, BAASMTC $5, x=0(.1) 20$

3. $8 \mathrm{D}, K_{n}(x), n=2(1) 12$, BAASMTC $5, x=0(.01) 10, \delta_{m}{ }^{2}$

4. $8 \mathrm{~S}, K_{n}(x), n=2(1) 20$, BAASMTC $5, x=.1(.1) 20, \delta^{2}$

5. $7-14 \mathrm{~S}, K_{n}(x), n=0(1) 10$, WATSON $3, x=.1(.1) 5$

6. 5S, $K_{n}(x), n=0(1) 10$, IsHERWOOD, $x=.2(.2) 5$

7. $6 \mathrm{~S}, T_{n}(x)=\left(\frac{1}{2} x\right)^{n} K_{n}(x) /\left[\pi^{3}\left(n-\frac{1}{2}\right)\right.$ !], ELDERTON, $n=0(1) 5$, $x=0(.1) 4(.5) 16(2) 40(5) 120 ; n=6(1) 11, x=0(.5) 16(2) 40(5) 120$

8. $8 \mathrm{~S}, x^{n} K_{n}(x), e^{x} K_{n}(x), n=2(1) 20$, BAASMTC $5, x=0(.1) 20$, $\delta^{2}$; also $n=2(1) 12$, $x=0(.01) 10, \delta_{m}^{2}$

9. 7D, $\log T_{n}(x)$ and $\rho$ (to $\left.5 \mathrm{D}\right)=K_{n-1}(x) / K_{n}(x)$, ELDERTON and $n=0(1) 5$, $x=0(.1) 4(.5) 16(2) 40(5) 120 ; n=6(1) 11, x=0(.5) 16(2) 40(5) 120$

10. $5 \mathrm{D}, 2\left(9+x^{2}\right) I_{3}(x) K_{3}(x)$, Yost, WhEELER \& BREIT, $x=.1, .2(.2) 1(.5) 5$

11. Graph, $K_{\theta}(x)$, McLachlan 2, p. $104,0<x<6$

12. Graphs, $e^{x} K_{0}(x)$, ZANSTRA, from which for $10^{-8}<x<12$ the function can be read to about 2D

13. Graph, $\frac{1}{2} x^{2} e^{-x}\left[K_{0}(x)+K_{1}(x)\right]$, DickmanN

$$
\text { C. } H_{n}^{(1)}(x)=J_{n}(x)+i Y_{n}(x), H_{n}^{(2)}(x)=J_{n}(x)-i Y_{n}(x)
$$

$K_{0}(x)=(\pi / 2) i H_{0}^{(1)}(i x)=-(\pi / 2) i H_{0}^{(2)}(-i x)$

$K_{1}(x)=-(\pi / 2) H_{1}^{(1)}(i x)=-(\pi / 2) H_{1}^{(2)}(-i x)$; see III $\mathbf{B}_{2} 3$

1. 7D, $\left|H_{n}{ }^{(1)}(x)\right|, n=0,1$, Watson $3, x=.02(.02) 16$. Compare II $\mathrm{A}_{z} 7,9,12$

2. $0^{\prime \prime} .01, \arg H_{n}^{(1)}(x)=\tan ^{-1}\left[Y_{n}(x) / J_{n}(x)\right], n=0,1$, WATSON $3, n=.02(.02) 16$

3. $4 \mathrm{~S}, 2\left|H_{1}{ }^{(1)}(x)\right|$, MORSE, p. $338\left(C_{0}\right), x=0(.1) .2(.2) 5$

4. 4-5S, $i H_{0}^{(1)}(i x)=(2 / \pi) K_{0}(x)=i H_{0}^{(2)}(-i x),-H_{1}^{(1)}(i x)=(2 / \pi) K_{1}(x)=H_{1}^{(2)}(-i x)$, JAHNKE \& EMDE $1_{2}-1_{b}, x=0(.01) .15 .99, \Delta$; also graphs

5. $5 \mathrm{D}, T(x)=\left[H_{0}^{(2)}(x)+i H_{1}{ }^{(2)}(x)\right] /\left[-H_{0}^{(2)}(x)+i H_{1}^{(2)}(x)\right]$, KüsSNER, tables of real and imaginary parts, $x=0, .002, .01, .02(.02) .7$

6. $8 \mathrm{D}, \log \left[\left(\frac{1}{2} \pi x\right)^{3}\left|H_{n}{ }^{(1)}(x)\right|\right], n=0(1) 6$, LODGE 4, T. I, $x=10(10) 100(100) 1000$; for $x=10, n=[4,5,6 ; 7 \mathrm{D}]$. Compare II $\mathrm{A}_{3}$

7. 7D, $\log \left[x\left|H_{n}{ }^{(1)}(x)\right|\right]^{3}, n=0(1) 6$, LODGE 4 , T. II, $x=10(10) 100(100) 1000$

8. Graph, $H_{1}^{(1)}(x) / H_{0}{ }^{(1)}(x)$, BuchноLz $2,0<x<12$

\section{Bessel Functions Whose Order is $\pm\left(n+\frac{1}{2}\right)$ $n+1$ a Positive Integer}

We shall first define Riccati-Bessel functions $S_{n}(x), C_{n}(x), E_{n}(x)$, using the notation of Doodson 1, 2, 3 (see $M T A C$, p. 71; for other notations see 
WATSON 3, p. 55-57). If $u^{2}=\frac{1}{2} \pi x, u>0\left[u^{2}\right.$ not as in X]

$$
\left\{\begin{array}{l}
S_{n}(x)=u J_{n+3}(x)=x^{n+1}\left(-\frac{d}{x d x}\right)^{n} \frac{\sin x}{x}, \\
C_{n}(x)=(-1)^{n} u J_{-n-1}(x)=x^{n+1}\left(-\frac{d}{x d x}\right)^{n} \frac{\cos x}{x}, \\
E_{n}(x)=C_{n}(x)-i S_{n}(x)=x^{n+1}\left(-\frac{d}{x d x}\right)^{n} \frac{e^{-i x}}{x}=i^{n} e^{-i x_{2} w_{n+1}}(-i x), \\
\left|E_{n}(x)\right|^{2}=C_{n}{ }^{2}(x)+S_{n}{ }^{2}(x)
\end{array}\right.
$$

These functions $S_{n}(x), C_{n}(x), E_{n}(x)$ are all solutions of the equation

$$
x^{2} y^{\prime \prime}+\left[x^{2}-n(n+1)\right] y=0
$$

which is transformable into Bessel's equation. All solutions of this equation are expressible in finite terms by means of algebraic and trigonometric functions of $x$. In particular,

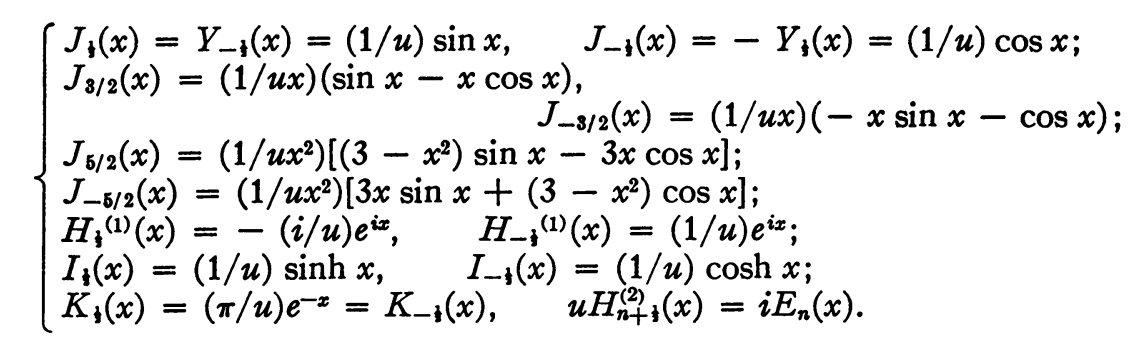

The zeros of $J_{3}(x)$ are those of $\sin x$ and the zeros of $J_{-4}(x)$ those of $\cos x$. The extension of this is that the zeros of $J_{3 / 2}(x)$ are the roots of $\tan x=x$, the zeros of $J_{-3 / 2}(x)$ are the roots of $\tan x=-x^{-1}$, and the zeros of $J_{5 / 2}(x)$ are the roots of $\tan x=3 x /\left(3-x^{2}\right)$.

In RAYleigh, The Theory of Sound (v. 1, 1877, p. 222-224; second ed., 1896, p. 278) we find that the solutions of problems connected with the lateral vibration of bars lead to the solution of the equations

$$
\begin{aligned}
& \cos x \cdot \cosh x=1, \\
& \cos x \cdot \cosh x=-1,
\end{aligned}
$$

which are equivalent to Greenhill's equations

$$
\begin{aligned}
& \tan \left(\frac{1}{2} x\right)=\mp \tanh \left(\frac{1}{2} x\right), \\
& \tan \left(\frac{1}{2} x\right)=\mp \operatorname{coth}\left(\frac{1}{2} x\right) .
\end{aligned}
$$

These may be written in the form

$$
\begin{aligned}
& J_{i}\left(\frac{1}{2} x\right) I_{-i}\left(\frac{1}{2} x\right) \pm J_{-i}\left(\frac{1}{2} x\right) I_{3}\left(\frac{1}{2} x\right)=0, \\
& J_{i}\left(\frac{1}{2} x\right) I_{1}\left(\frac{1}{2} x\right) \pm J_{-3}\left(\frac{1}{2} x\right) I_{-3}\left(\frac{1}{2} x\right)=0,
\end{aligned}
$$


and so are of the same general type as an equation which occurs in the theory of the vibration of a clamped circular plate. This is seen by writing $\left(4^{\prime \prime}\right)$ in the equivalent form

$$
J_{3 / 2}\left(\frac{1}{2} x\right) I_{1}\left(\frac{1}{2} x\right)+J_{1}\left(\frac{1}{2} x\right) I_{3 / 2}\left(\frac{1}{2} x\right)=0
$$

when the lower sign is taken.

For (4) and (5) RAYLEIGH found general formulae for the roots. STERN 1, p. 34. 35, gave the value of $x_{1}$ in (4) and (5) to 8D. The results of RAYLEIGH are listed also in AdAms 1.

As LAMB points out in The Dynamical Theory of Sound (1910, p. 129-130) some numerical results for the transverse vibrations of a bar were given by Euler (1740), Lissajous (1850) and SeEbeck (1848).

The Sine, Cosine, and Exponential Integrals are defined by

$$
\operatorname{Si}(x)=\int_{0}^{x} \sin x d x / x, \quad C i(x)=\int_{\infty}^{x} \cos x d x / x, \quad E i(x)=\int_{-\infty}^{x} e^{x} d x / x
$$

SCHAFHEITLIN ${ }^{1}$ has shown that if

then

$$
V_{r}(x)=(\partial / \partial r) J_{r}(x), \quad W_{r}(x)=(\partial / \partial r) Y_{r}(x),
$$

$$
\begin{aligned}
& \operatorname{Si}(2 x)=-\frac{1}{2} \pi \cos 2 x-u \cos x V_{t}(x)-u \sin x W_{t}(x) \text {, } \\
& C i(2 x)=\frac{1}{2} \pi \sin 2 x+u \sin x V_{1}(x)-u \cos x W_{1}(x) \text {, } \\
& E i(2 x)=-\frac{1}{2} \pi i e^{2 i x}-u e^{i x}\left[i V_{i}(x)+W_{i}(x)\right] \text {. }
\end{aligned}
$$

There have been many tables of these integrals, the most elaborate of $\operatorname{Si}(x)$ and $C i(x)$ being that of the Mathematical Tables Project (New York, 1942) where there is a full bibliography. We shall not list other tables of this type, but we may note one more result, due to LOMMEL 3, p. 550, viz:

$$
\operatorname{Si}(2 x)=\int_{0}^{2 x} \sin t d t / t=\pi \sum_{n=0}^{\infty} J_{n+\frac{1}{3}}^{2}(x) .
$$

Tables involving half odd integer values of $n$ have been prepared by LODGE 4,5 on the basis of the relation

$$
J_{n}(x)=(R / u) \cos \left(x+\alpha-\frac{1}{4} \pi-\frac{1}{4} n \pi\right)
$$

discussed in XIII.

Other functions of importance are the Spherical Bessel Functions or Stokes's Functions, $y=(\pi / 2 x)^{\frac{1}{3}} J_{n+1}(x)$. These functions satisfy the differential equation

$$
x^{2} y^{\prime \prime}+2 x y^{\prime}+\left[x^{2}-n(n+1)\right] y=0,
$$

of which the two solutions are

$$
\begin{aligned}
j_{n}(x) & =(\pi / 2 x)^{\frac{1}{3}} J_{n+1}(x)=S_{n}(x) / x, & n_{n}(x) & =(\pi / 2 x)^{3} Y_{n+1}(x), \\
j_{-n}(x) & =(-1)^{n} n_{n-1}(x), & n_{-n}(x) & =(-1)^{n-1} j_{n-1}(x) .
\end{aligned}
$$


From this last relation a table of $j_{n}(x), n_{n}(x)$ for $n=0(1) 2$ is also a table of these functions for $n=-3(+1)-1$. Some special cases of the functions are as follows:

$$
\begin{aligned}
& j_{0}(x)=\sin x / x, \quad n_{0}(x)=-(\cos x) / x, \\
& j_{1}(x)=\left(\sin x / x^{2}\right)-(\cos x / x), \quad n_{1}(x)=-[(\sin x) / x]-(\cos x) / x^{2}, \\
& j_{2}(x)=\left[\left(3 / x^{3}\right)-(1 / x)\right] \sin x-\left(3 / x^{2}\right) \cos x, \\
& n_{2}(x)=-\left(3 / x^{2}\right) \sin x-\left[\left(3 / x^{3}\right)-(1 / x)\right] \cos x .
\end{aligned}
$$

MORSE and HAurvitz tabulated

$$
\begin{aligned}
D_{n} & =\left\{\left[j_{n}(x)\right]^{2}+\left[n_{n}(x)\right]^{2}\right\}^{\frac{1}{3}}, \text { and } D_{n}^{\prime}=\left[\left(d j_{n}(x) / d x\right)^{2}+\left(d n_{n}(x) / d x\right)^{2}\right]^{\frac{1}{3}} \\
\delta_{n} & =-\tan ^{-1}\left[j_{n}(x) / n_{n}(x)\right], \text { and } \delta_{n}^{\prime}=\tan ^{-1}\left\{\left[d j_{n}(x) / d x\right] /\left[d n_{n}(x) / d x\right]\right\} ; \\
D_{0} & =-1 / x ; \quad D_{1}=\left(1+x^{2}\right)^{\frac{1}{2} / x^{2}=D_{0},} \text { and } D_{1}^{\prime}=\left(4+x^{4}\right)^{\frac{3}{3}} / x^{3} .
\end{aligned}
$$

RAYLEIGH and PARIS have tabulated

$$
\psi_{n}(x)=(-1)^{n}(2 n+1) ! !\left(\frac{1}{x} \frac{d}{d x}\right)^{n} \frac{\sin x}{x}=(2 n+1) ! !\left(\frac{\pi}{2 x^{2 n+1}}\right)^{!} J_{n+1}(x),
$$

and

$$
\begin{gathered}
\Psi_{n}(x)=(-1)^{n}(2 n+1) ! !\left(\frac{1}{x} \frac{d}{d x}\right)^{n} \frac{\cos x}{x}=(2 n+1) ! !\left(\frac{\pi}{2 x^{2 n+1}}\right)^{\frac{1}{2}} J_{n-1}(x) ; \\
E_{n}(x)=\Psi_{n}(x)+i \psi_{n}(x) .
\end{gathered}
$$

PARIS gave also a table for functions defined as follows:

$$
M_{n}(x)=\frac{\psi_{n}(x)}{-E_{n}(x)}, \quad N_{n}(x)=\frac{(2 n+1) \psi_{n-1}(x)-n \psi_{n}(x)}{-(2 n+1) E_{n-1}(x)+n E_{n}(x)} .
$$

The tables and notation of MIE call for some preparatory remarks. His $K_{n}(-x)$ is connected with $E_{n}(x)$ by the relation $E_{n}(x)=i^{n} K_{n}(-x)$; and his $I_{n}(x)$ is our $S_{n}(x)$. Hence his $a_{n}, p_{n}$ may be defined as follows:

$$
\begin{gathered}
a_{n}=(2 n+1) \frac{\alpha S_{n}^{\prime}(\beta) S_{n}(\alpha)-\beta S_{n}^{\prime}(\alpha) S_{n}(\beta)}{\beta E_{n}^{\prime}(\alpha) S_{n}(\beta)-\alpha E_{n}(\alpha) S_{n}^{\prime}(\beta)}, \\
p_{n}=(2 n+1) \frac{\alpha S_{n}^{\prime}(\alpha) S_{n}(\beta)-\beta S_{n}(\alpha) S_{n}^{\prime}(\beta)}{\alpha E_{n}^{\prime}(\alpha) S_{n}(\beta)-\beta E_{n}(\alpha) S_{n}^{\prime}(\beta)} \\
\alpha=2 \pi m_{0} \rho / \lambda=2 \pi \rho / \lambda^{\prime}, \quad \beta=2 \pi m \rho / \lambda=m \alpha / m_{0}=m^{\prime} \alpha .
\end{gathered}
$$

WANNIER has a table of $j_{m, n}(x)=x e^{-x} \int_{-1}^{+1} e^{z x} P_{m}(z) P_{n}(z) d z$. By the formula of Adams

$$
P_{m}(z) P_{n}(z)=\Sigma A_{r} P_{r}(z) \quad \text { and } \quad \int_{-1}^{+1} e^{z x} P_{n}(z) d z=(2 \pi)^{\frac{1}{2}} x^{-\frac{1}{2}} I_{n+z}(x) .
$$

Thus $j_{m, n}(x)$ is expressible in terms of the functions $I_{n+\xi}(x)$. Wannier has also tables of series involving $j_{0, n}^{2}(x)$.

1 P. Schafheitlin, Berlin Math. So., Sitzungsb., v. 8, 1909, p. 64. 
A. $J_{ \pm(n+1)}(x)= \pm(-1)^{n} Y_{\mp(n+1)}(x)$

1. 12D, Airey 23

\begin{tabular}{|c|c|c|c|c|c|c|c|c|}
\hline$x$ & $\begin{array}{c}n+\frac{1}{2} \\
n=\end{array}$ & $\begin{array}{c}-n-\frac{1}{2} \\
n=\end{array}$ & $x$ & $\begin{array}{c}n+\frac{1}{2} \\
n=\end{array}$ & $\begin{array}{c}-n-\frac{1}{2} \\
n=\end{array}$ & $x$ & $\begin{array}{c}n+\frac{1}{2} \\
n=\end{array}$ & $\begin{array}{c}-n-\frac{1}{2} \\
n=\end{array}$ \\
\hline 1 & $0(1) 11$ & 0 & 8 & $0(1) 26$ & $0(1) 9$ & 15 & $0(1) 37$ & $0(1) 17$ \\
\hline & $0(1) 14$ & $0(1) 2$ & 9 & $0(1) 28$ & $0(1) 10$ & 16 & $0(1) 39$ & $0(1) 18$ \\
\hline 3 & $0(1) 17$ & $0(1) 3$ & 10 & $0(1) 30$ & $0(1) 11$ & 17 & $0(1) 40$ & $0(1) 19$ \\
\hline & $0(1) 19$ & $0(1) 4$ & 11 & $0(1) 31$ & $0(1) 13$ & 18 & $0(1) 42$ & $0(1) 20$ \\
\hline 5 & $0(1) 21$ & $0(1) 5$ & 12 & $0(1) 33$ & $0(1) 14$ & 19 & $0(1) 43$ & $0(1) 21$ \\
\hline & $0(1) 23$ & $0(1) 7$ & 13 & $0(1) 34$ & $0(1) 15$ & 20 & $0(1) 44$ & $0(1) 22$ \\
\hline & $0(1) 25$ & $0(1) 8$ & 14 & $0(1) 36$ & $0(1) 16$ & & & \\
\hline
\end{tabular}

2. 9-12D, HAYASHI $1, n=1,0 ; x=[0(.01) 1.99 ; 12 \mathrm{D}]$, [2(.01)9.89; 9D], [9.9(.01)10(.1)20(1) $100 ; 12 \mathrm{D}]$

3. $6 \mathrm{D}$, Watson $3, n=0(1) 6 ; x=0$ (1) 50 . And for $J_{n+3}(x), n=7(1) 12, x=2(1) 20$; and for $J_{n+3}(x), n=13(1) 18, x=5(1) 20$

4. 6D, LommeL $3, n=0(1) 6 ; x=0$ (1)50. And for $J_{n+3}(x), n=7(1) 13, x=0$ (1)20; $n=14(1) 20, x=5(1) 20 ; n=21(1) 27, x=10(1) 20 ; n=28(1) 34, x=15(1) 20$

5. $6 \mathrm{D}$, AIREy $24, n=0 ; x=0(.02) 20$

6. 5D, HaYASHI $2, n=0,1 ; x=0(.01) 1(.1) 10(1) 100$

7. $4 \mathrm{~S}$, JAHNKE \& EMDE $1_{1}-1_{5}, n=0(1) 6 ; x=0(1) 50$

8. 4 S, JAHNKE \& EMDE $1_{2}-1_{5}, n=0(1) 6$ and others up to 18 , for $J_{n+3}(x) ; x=1(1) 24$

9. $4 \mathrm{D}$, DINNIK $4,6,7, n=0,1,2 ; x=0(.2) 8$

10. $4 \mathrm{D}$, DiNNIK $14, n=0 ; x=0(.1) 15$

11. 9 duodecimals, TERRY, $n=0, J_{1}(x)$ only, in duodecimals for $x=0\left(12^{-1}\right) 1, \delta^{2}$

12. 7D, $J_{n}\left(j_{0, s}\right)$, Buchrolz $3, n=\frac{1}{2}\left(\frac{1}{2}\right) 10 \frac{1}{2} ; s=1 ; n=\frac{1}{2}\left(\frac{1}{2}\right) 16, s=2 ; n=\frac{1}{2}\left(\frac{1}{2}\right) 20 \frac{1}{2}, s=3$. The results are shown graphically on p. 161 for $1<r<13$.

\section{B. Riccati-Bessel Functions $S_{n}(x), C_{n}(x)$}

1. 7D or $7 \mathrm{~S}, S_{n}(x), C_{n}(x), S_{n}{ }^{\prime}(x), C_{n}{ }^{\prime}(x)$, and the logarithms of their absolute values, Doodson $1, n=0(1) N, N$ varying from 7 to 23 , for $x=1$ (1)10. Doodson $2, n=0(1) N$; $N=8, x=1.1(.1) 14 ; N=9, x=1.5(.1) 1.8 ; N=10, x=1.9$. Doodson $3, n=0(1) N$, $N$ varying from 3 to 7 , for $x=.1(.1) .9$

2. 7D, $S_{n}(x), S_{n}{ }^{\prime}(x)$, BLUMER $1, n=0(1) 7, x=2.5$

3. $4 \mathrm{D}$ (mostly), $S_{n}(x), S_{n}{ }^{\prime}(x), x S_{n}{ }^{\prime}(x) / S_{n}(x)$, Yost, WheEler \& BREIT $1, n=0(1) 4$, $x=.1(.1) 5.6$

4. 7D or 7S, $E_{n}{ }^{2}(x)=S_{n}{ }^{2}(x)+C_{n}{ }^{2}(x),\left[E_{n}{ }^{\prime}(x)\right]^{2}=\left[S_{n}{ }^{\prime}(x)\right]^{2}+\left[C_{n}{ }^{\prime}(x)\right]^{2}$, and their logarithms, Doodson 1, $n=0(1) N, N$ varying from 4 to $15, x=1(1) 10$. Doodson 2, $n=0(1) N, N=3, x=1.1(.1) 1.3 ; N=4, x=1.4(.1) 1.9$. Doodson 3, $n=0(1) N$, $N$ varying from 3 to 7 , for $x=.1(.1) .9$

5. 5D, $M_{p} S_{n}(x) C_{n}(x) /\left[E_{n}(x)\right]^{2}$, Proudman, Doodson \& Kennedy, $n=1(1) 17$; also $M_{p} S_{n}{ }^{2}(x) /\left[E_{n}(x)\right]^{2}, n=1(1) 14 ; M_{p} S_{n}{ }^{\prime}(x) C_{n}{ }^{\prime}(x) /\left[E_{n}{ }^{\prime}(x)\right]^{2}, n=1(1) 17 ; M_{p}\left[S_{n}{ }^{\prime}(x)\right]^{2} /\left[E_{n}{ }^{\prime}(x)\right]^{2}$, $n=1(1) 14 ; 8$ tables, $x=10$ in each, $p=1,2$, and $M_{1}=(-1)^{n}(2 n+1) P_{n}{ }^{\prime}(\cos \theta) / n(n+1)$, $M_{2}=(-1)^{n}(2 n+1) P_{n}(\cos \theta), \theta=0\left(10^{\circ}\right) 30^{\circ}\left(15^{\circ}\right) 60^{\circ}\left(10^{\circ}\right) 90^{\circ}$

6. $4 \mathrm{D}$ or $5 \mathrm{~S}, S_{n}(x), C_{n}(x)$, STENZEL 1 .

$$
\begin{array}{rlrl}
x=2.2(.2) 2.8, & & n=0(1) 7 \\
3.2(.2) 3.8, & & n=0(1) 8 \\
& 4.2(.2) 4.8, & & n=0(1) 9 \\
& 5.2(.2) 5.8, & & n=0(1) 11
\end{array}
$$

$$
\begin{aligned}
x=6.2(.2) 6.8, & & n=0(1) 12 \\
7.2(.2) 7.8, & & n=0(1) 13 \\
8.2(.2) 8.8, & & n=0(1) 14 \\
9.2(.2) 9.8, & & n=0(1) 15
\end{aligned}
$$

7. $4 \mathrm{D}, n S_{n}(10), 10 S_{n+1}(10), U_{n}(10), n C_{n}(10), 10 C_{n+1}(10), V_{n}(10), U_{n}(10) / V_{n}(10)$, $\left[U_{n}(10) / V_{n}(10)\right] /\left[1+U_{n}^{2}(10) / V_{n}^{2}(10)\right], n=0(1) 15$, STENZEL 1.

$\left[U_{n}(x)=x S_{n+1}(x)-n S_{n}(x), V_{n}(x)=x C_{n+1}(x)-n C_{n}(x)\right]$ 


\section{Spherical Bessel Functions $j_{n}(x)=(\pi / 2 x)^{i} J_{n+\xi}(x)$, etc.}

1. $10 \mathrm{D} ; 8 \mathrm{~S}, j_{n}(x)$, NYMTP $9, \pm n=0(1) 21, x=0(.01) 10$, with second and fourth central differences; also extended $n>21$ and $\langle-21, x\rangle 10$

2. $5 \mathrm{~S}, j_{n}(x)$, NYMTP $2, \pm n=1(1) 10, x=0(.1) 10$

3. $5 \mathrm{D} ; 5 \mathrm{~S}, j_{n}(x)$, MoRse \& HaURvitz, $n=-9(1)+8, x=0(.1) \cdot 2(.2) 9 ; n=-3(1)+2$, $x=0(.1) .2(.2) 10$

4. $5 \mathrm{~S}, j_{n}(x)$, MORSE, p. $335, n=-3(1)+2, x=0(.1) .2(.2) 8$

5. $6 \mathrm{D}, H(n, 0, x)=(2 n+1) ! j_{n}(x) /\left[n !(2 x)^{n}\right], H^{\prime}(n, 0, x)$, Lowan \& Horenstein, $n=0(1) 3, x=0(1) 10$

6. $8 \mathrm{D}, j_{0}(x)=\sin x / x, n_{0}(x)=\cos x / x$, HAYASHI $1, x=0(.01) 10(.1) 20(1) 100$. In LoMmeL 3 , p. 651 there is a table, to $6 \mathrm{D}$, of $j_{0}(x)$ and of $j_{0}{ }^{2}(x)$, also the first 17 maxima of $j_{0}(x)$ (roots of the equation $J_{3 / 2}(x)=0$ ) as well as the values of $j_{0}(x)$ and $j_{0}{ }^{2}(x)$ at these maxima. No other references to tables of $j_{0}(x)$ and $n_{0}(x)$ will be listed. As to tables of $\log j_{0}(x)$ a reference may be given to $M T A C$, p. 83-85.

7. $4 \mathrm{~S}, D_{n}{ }^{\prime}, n=0(1) 4$, MORSE, p. $339, x=0(.1) .2(.2) 5$; also to $0^{\circ} .1$, var. $\delta_{n}{ }^{\prime}, n=0(1) 4$

8. $4 \mathrm{D}, D_{n}, D_{n}^{\prime}$, MoRSe \& HaURvitz, $n=-3(1)+2, x=0(.1) .2(.2) 10$

9. $1^{\prime}, \delta_{n}, \delta_{n}{ }^{\prime}$, Morse \& HaURvitz, $n=0(1) 2, x=0(.1) .2(.2) 10$

10. 5-6S or 5-6D, $\Psi_{n}(x), \psi_{n}(x)$, RAYLEIGH $7, n=0(1) 6$ (some values missing between 3 and 6$), 8 x=8,12(2) 18(3) 27$

11. 5S, $\Psi_{n}(x) ; \psi_{n}(x)$, PARIS, $n=0(1) 4 ; 0(1) 6, x=1.25$

12. $5 \mathrm{D}, M_{n}, N_{n}$, PARIS, $n=1(1) 3, x=1 ; n=1(1) 4, x=1.5 ; n=1(1) 5, x=1.75$; $n=1(1) 5, x=2$

\section{D. $J_{n}(x), I_{n}(x), K_{n}(x), n$ half an odd integer, Miscellaneous}

1. $5 \mathrm{D},\left(\frac{1}{2} 9 \pi\right) x^{-8} J_{3 / 2}^{2}(x)$, BLUMER $2, x=2 \alpha \cos \left(\frac{1}{2} \gamma\right)$,

$\gamma=0\left(10^{\circ}\right) 30^{\circ}\left(15^{\circ}\right) 60^{\circ}\left(10^{\circ}\right) 120^{\circ}\left(15^{\circ}\right) 150^{\circ}\left(10^{\circ}\right) 180^{\circ}, \alpha=5$. Also to 5D, KLEINERT, for $\gamma=0,90^{\circ}\left(10^{\circ}\right) 180^{\circ}, \alpha=9.9952,12.482,13.862,17.327$

2. $4-5 \mathrm{D},\left(\frac{1}{2} 9 \pi\right) x^{-3} J_{3 / 2}^{2}(x)$, GANS $3, x=0(1) 12$

3. $3 \mathrm{D}, \Lambda_{\mathbf{3} / 2}(x) / \Lambda_{\mathfrak{\xi}}(x), x=r \pi+\theta$, Chapman \& Whitehead, $r=0(1) 3, \theta=\pi / 10(\pi / 10) \pi$; also $\Lambda_{7 / 2}(x) / \Lambda_{5 / 2}(x), r=2(1) 7, x^{2}=\frac{1}{2} r \pi+\theta, \theta=0(\pi / 10) 5 \pi / 10$. $\left[\Lambda_{n+3}(x)=\left(\frac{1}{2} x\right)^{-(n+\xi)}\left(n+\frac{1}{2}\right) ! J_{n+3}(x)\right.$; hence $\Lambda_{3}(x)=\sin x / x$, $\left.\Lambda_{3 / 2}(x)=\left(3 / x^{2}\right)\{\sin x / x-\cos x\}, \Lambda_{3 / 2}(x) / \Lambda_{1 / 2}(x)=\left(3 / x^{2}\right)(1-x \cot x)\right]$

4. 8D; 7D, $\log \left[\frac{1}{2} \pi x\left\{J_{n}^{2}(x)+Y_{n}^{2}(x)\right\}\right]^{3} ; \log \left[x\left\{J_{n}^{2}(x)+Y_{n}^{2}(x)\right\}\right]^{3}, n=0\left(\frac{1}{2}\right) 6 \frac{1}{2}$, LODGE 4, $x=10(10) 100(100) 1000$

5. 7D, $\log \left[8 x a_{n} /\left(4 n^{2}-1\right)\right], n=0\left(\frac{1}{2}\right) 6 \frac{1}{2}$, LODGE $5, x=10(10) 100(100) 1000$.

$\left[a_{n}=\frac{1}{2}\left(n+\frac{1}{2}\right) \pi-x+\tan ^{-1}\left\{Y_{n}(x) / J_{n}(x)\right\}\right]$

6. $6 \mathrm{D}, J_{n}(x), n= \pm x, \pm(x-1)$, AIREY 20 , p. 290-291, $x=\frac{1}{2}(1) 9 \frac{1}{2}$

7. $6 \mathrm{D}, J_{n}(x), n=x \pm \frac{1}{2}$, AIREY $31, x=1$ (1)20. The admirably compact forms of statement in nos. 6-7 are those of the Liverpool Index

8. $6 \mathrm{D}, J_{ \pm \frac{1}{3}}^{(p-1)}(x) / 2 . p$ !, $p=1(1) 6$, LoMmeL 3, p. 649-650, $x=1(1) 50$

8A. $3 \mathrm{D}, \frac{1}{2} a_{1} / \alpha^{3}, \alpha^{2}=0(.2) 1(.5) 2,2.6$, MiE, p. 420 , when $\lambda=420,450,500,525$; for $\lambda=550,600,650$ the set of values ends with 2.5 instead of 2.6. On p. 417 Mie has a table of values of $m, m^{\prime 2}$ and $\lambda^{\prime}$ corresponding to these values of $\lambda$. On p. $418\left|a_{1}\right|^{2}$, for gold is given to $3 \mathrm{~S}$ for the foregoing values of $\lambda$. Among other results are the following tables of: on p. $421,(4 \pi / \lambda) \alpha^{3}\left|a_{1}\right|^{2}$, for $\alpha^{2}=[.2(.2) 1(.5) 2.5 ; 3-4 \mathrm{~S}]$; on p. $426, \frac{1}{2} a_{\alpha} / \alpha^{3}$, for $\alpha^{2}=$ [small, 1, 2, 2.5; 3D]; on p. $426, \frac{1}{2} \rho_{1} / \alpha^{3}$, for $\alpha^{2}=$ [small, $1,2,1.5 ; 3 \mathrm{D}$ ].

9. 4-6S, $I_{3}(x)=\sinh x /\left(\frac{1}{2} \pi x\right)^{4}$, DiNNIK $14, x=0(.1) 15$

10. $5-6 \mathrm{~S}, I_{-3}(x)=\cosh x /\left(\frac{1}{2} \pi x\right)^{3}$, DINNIK $14, x=0(.1) 7$

11. $6 \mathrm{D}, \frac{1}{2}\left[I_{-1}(x)-I_{1}(x)\right]=e^{-x} /[2 \pi x]^{3}$, WRINCH \& WRINCH $1, x=1(1) 13$

12. $6 \mathrm{~S}, \frac{1}{2}\left[I_{3}(x)+I_{-3}(x)\right]=e^{x} /[2 \pi x]^{3}$, WRINCH \& WRINCH, $1,2, x=1(1) 40$

13. $7 \mathrm{D},(2 \pi x)^{3} e^{-x} I_{n}(x), n=0\left(\frac{1}{2}\right) 1 \frac{1}{2}$, OKAYA $1,1 / x=0(.01) .1$

14. $4 \mathrm{D},\left(\frac{1}{2} \pi x\right)^{\frac{3}{3}} e^{-x}\left[I_{n-1}(x)+I_{n}(x)\right]$, (a) $n=1\left(\frac{1}{2}\right) 2$, EDdington, OKAYA 1 and (b) $n=1\left(\frac{1}{2}\right) 2 \frac{1}{2}$, SMART, $(2 x)^{3}=0(.1) 2$. Also $(\mathrm{a}, \mathrm{b}),|P|=\left[I_{3}(x)+I_{3 / 2}(x)\right] /\left[I_{0}(x)+I_{1}(x)\right]$ to $4 \mathrm{D}$, and $\zeta=\left[I_{0}(x)+I_{1}(x)\right]^{2} /\left[I_{3}(x)+I_{3 / 2}(x)\right]$ to $3 \mathrm{D},(2 x)^{\xi}=0(.1) 2$ 
15. 5D, $T(1,1, x)=x \pi^{3} e^{-j x^{2}} I_{\xi}\left(\frac{1}{2} x^{2}\right)$, HeAtley 2 , p. $29, x=0(.1) 2(.2) 3$

16. 7D, $A_{2 n+1}=(\pi x)^{\frac{1}{3}} e^{-j x} I_{n+1}\left(\frac{1}{2} x\right)$, IvORY, $x=2 m=10, n=0$ (1) 12

17. $1-7 \mathrm{D}, a_{2 n+1}=w_{n+1}(x)$, IVORY, $x=2 m=10, n=[0(1) 6 ; 1-6 \mathrm{D}], n=[7(1) 12 ; 7 \mathrm{D}]$

18. $6 \mathrm{D}, B_{2 n+1}=w_{n+3}(-x)$, essentially $(x / \pi)^{3} e^{j x} K_{n+1}\left(\frac{1}{2} x\right)$, IVORY, $x=2 m=10, n=0(1) 12$

19. $4 \mathrm{D}, j_{m, n}(x), m=0(1) 5, n=0(1) 5$, WANNIER, $x=5,10(10) 100$

20. $6 \mathrm{D}, T_{n}(x)$, ElDERTON, $n=0\left(\frac{1}{2}\right) 5 \frac{1}{2}, \quad x=0(.1) 4(.5) 16(2) 40(5) 120 ; \quad n=6 \frac{1}{2}\left(\frac{1}{2}\right) 11 \frac{1}{2}$, $x=0(.5) 16(2) 40(5) 120$. Also to 7D $\log T_{n}(x)$; and to $5 \mathrm{D}, n \neq 0, \rho=K_{n-1}(x) / K_{n}(x)$. Compare III $\mathrm{B}_{2} 7,13 ; \mathrm{B}_{3} 7,9$

21. $5 \mathrm{D}$ or $5 \mathrm{~S}, x^{n+1} \chi_{n}(x)\left[=-i\left(\frac{1}{2} \pi x\right)^{4} H_{n+1}^{(2)}(x)\right], \quad D_{n}(x)=10^{n+1}\left[\chi_{n-1}(x)-(n+1) \chi_{n}(x)\right]$, RAYLEIGH $6, x=10, n=0(1) 22$

22. $5 \mathrm{D}, i^{n}(2 n+1) D_{n}^{-1}, n=0(1) 22$, RAYLEIGH 6

See also VIII, $\partial J_{n}(x) / \partial n, \partial^{2} J_{n}(x) / \partial n^{2}$

\section{E. Zeros}

1. 12D, first 53 zeros of $J_{n}(x), Y_{n}(x), n=1 \frac{1}{2}\left(\frac{1}{2}\right) 10 \frac{1}{2}$, BAASMTC 9

2. $4 \mathrm{D}, J_{n}(x), 2 n=1,2,3$, LAMB 1 , first 3 zeros

3. $4 \mathrm{~S}, J_{n}(x), n=7 \frac{1}{2}(1) 9 \frac{1}{2}, 12 \frac{1}{2}(1) 14 \frac{1}{2}$, KALÄHNE 2 , p. 85

4. 4D, first 11 zeros of $J_{3 / 2}(x)$, EULER; compare $M T A C$, p. 203

5. $8 \mathrm{D}$, first zero of $J_{3 / 2}(x)$, STERN

6. 6D, first 17 zeros of $J_{3 / 2}(x)$, LoMmeL 3

7. $4 \mathrm{D}$, first 17 zeros of $J_{3 / 2}(x)$, JAHNKE \& EMDE $1_{1}, 1_{2}, 1_{6}$, and EMDE 2

8. $6 \mathrm{D}, 18$ th to 36 th zeros of $J_{3 / 2}(x)$, SILBERSTEIN

9. $4 \mathrm{D}$, first 36 zeros of $J_{3 / 2}(x)$, HaYashi 2, p. 52

10. $4 \mathrm{D}$, first 7 zeros of $J_{5 / 2}(x)$, LAMB 1

11. $4 \mathrm{D}$, first 2 zeros of $J_{\mathrm{s} / 2}(x)$, GaNs 2

12. $3 \mathrm{D} ; 2 \mathrm{D}, J_{-3 / 2}(x) ; J_{-6\}}^{\prime}(x)$, first zeros, AIREY 15 ; also first zero of $J_{-5 / 2}(x)$ expressed in terms of $r(3 D)$ and $\theta$

13. $8 \mathrm{D}$, first zeros of $J_{i}(x) I_{\mp\}}(x) \pm J_{-j}(x) I_{ \pm\}}(x)$, STERn. Also to $6 \mathrm{D}$, first 5 or 6 zeros, Adams. Also, to $4 \mathrm{D}$, first 5 or 6 zeros, Hayashi 2, p. 52

14. 4D (mostly), $J_{n}(x) Y_{n}(k x)-J_{n}(k x) Y_{n}(x), 2 n=1,3,5$, KALÄHNE 1, and KALÄHNE 2, p. 68 , first 6 zeros, $x_{n, s}$, and $(k-1) x_{n, s}, 10 k=12,15,20$. Also, KALÄHNE 2, p. 80-81, $(k-1) x_{n, .}$ for $n=3 / 2, k=[1,1.2(.1) 1.5,12 / 3,1.8,2,21 / 3,3(1) 11,19,39 ; 3-5 S]$; and $n=5 / 2, k=[1,1.2,1.5,2(1) 11,19,39 ; 3-5 S]$. Most of these results are in JAHNKE \& EMDE $1_{1}-1_{5}$

15. If $x_{n}$ represents the zeros of $J_{3 / 2}(x)$, exact values are given for $\sum_{n=1}^{\infty} x_{n}{ }^{-p},(p=2,4)$, and $\sum_{n=1}^{\infty} 1 /\left(x_{n}^{8} \sin x_{n}\right)$, Forsyth

16. $4 \mathrm{D}, \psi_{n-1}(x), n=1(1) 3$, LAMB 2,3 zeros for each $n$.

\section{F. Graphs}

1. $J_{ \pm(n+1)}(x)$, JAHNKE \& EMdE $1_{1}-1_{2}$ (figs. 116-118) $n=0,1,6 ; J_{-(n+1)}(x), 1_{3}-1_{5}$ (fig. 86), $n=0(1) 6$

2. $J_{ \pm(n+1)}(x), n=0,1$, HaYAShI 2 , p. $101,0<x<13$

3. $1+\left(2 \pi / x^{3}\right) J_{3 / 2}(x)$, FRANZ, $0<x<4 \pi$

4. $(3 \pi / 16)\left[3 J_{1}(x) J_{-3}(x)+J_{3 / 2}(x) J_{-3 / 2}(x)\right]$, FRANZ, $0<x<2 \pi$

5. $I_{ \pm\}}(x)$, DiNNIK 14, p. $9,0<x<5$

6. $j_{\text {on }}(x)=x e^{-x} \int_{-1}^{+1} e^{x z} P_{n}(z) d z=(2 \pi x)^{3} e^{-x} I_{n+1}(x)$, WANNIER, $n=2(1) 37,0<x<100$

7. $\frac{1}{2} x\left[2 w_{n+1}(i x) w_{n+1}(-i x)+w_{n+1}(i x) w_{n+1}^{\prime}(-i x)+w_{n+1}^{\prime}(i x) w_{n+1}(-i x)\right]$, BACKHAUS 1, $n=0(1) 4,0<x<8$

8. $U_{n}(x), V_{n}(x)$ [compare IV B 7], Stenzel $1, x=4, n=0(2) 8 ; x=10, n=0$ (2)14; $x=20, n=0(2) 26 ; x=40, n=0(2) 52$ 


\section{Bessel Functions OF Order $\pm 1 / 3, \pm 2 / 3$}

A Bessel function of order $1 / 3$ occurred in a letter written in 1703 by Jakob (James) Bernoull to Leibniz (see Watson 3, p. 1-3), but a systematic study of these functions was not made until over one hundred years after this.

In a memoir on the intensity of light, published in 1838 by G. B. AIrY, he introduced the integral

$$
A(m)=\int_{0}^{\infty} \cos \frac{1}{2} \pi\left(w^{3}-m w\right) d w
$$

which is a solution of the linear differential equation

$$
y^{\prime \prime}=\frac{1}{3} x y \text {. }
$$

While this integral is known as Airy's Integral, another form was suggested by HAROLD JEFFreys and adopted by the BAASMTC, which has a volume of tables of such integrals in process of publication. J. C. P. MILLER, the chief author of the volume, has kindly responded to our request for information in this regard and has sent to us interesting material mainly indicated in AIRY 5, BAASMTC 8, RAYLEIGH 7, and in the following quotation:

"The complete solution of the differential equation $y^{\prime \prime}=x y$ may be written as $y=a A i(x)+b B i(x)$, in which $a$ and $b$ are constants, and we define

$$
\left\{\begin{array}{l}
A i(x)=\frac{1}{\pi} \int_{0}^{\infty} \cos \left(\frac{1}{3} t^{3}+x t\right) d t \\
B i(x)=\frac{1}{\pi} \int_{0}^{\infty}\left\{\exp \left(-\frac{1}{3} t^{3}+x t\right)+\sin \left(\frac{1}{3} t^{3}+x t\right)\right\} d t .
\end{array}\right.
$$

We may also write

$$
\begin{cases}A i(x)=F(x) \sin \chi(x), & A i^{\prime}(x)=G(x) \sin \psi(x), \\ B i(x)=F(x) \cos \chi(x), & B i^{\prime}(x)=G(x) \cos \psi(x) .\end{cases}
$$

The four auxiliary functions are monotone for $-\infty<x<0$.

Writing $\xi=\frac{2}{3} x^{3 / 2}$, we may express these functions in terms of Bessel Functions of order $\pm \frac{1}{3}, \pm \frac{2}{3}$, as follows:

(5)

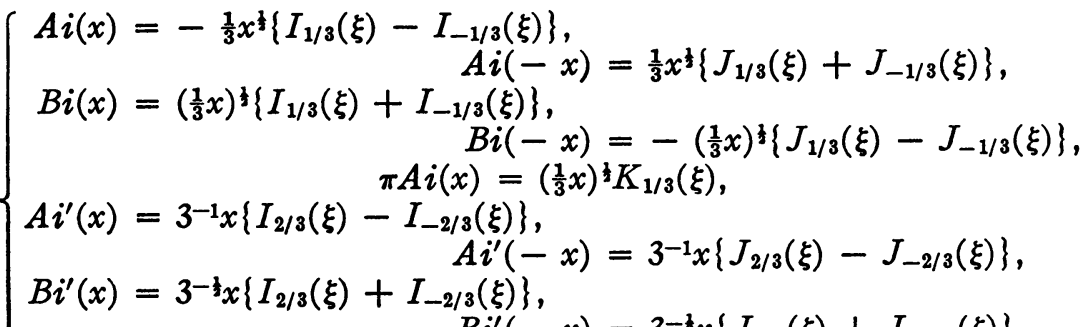

$$
\begin{aligned}
& B i^{\prime}(-x)=3^{-k} x\left\{J_{2 / 3}(\xi)+J_{-2 / 3}(\xi)\right\} \text {, } \\
& \pi A i^{\prime}(x)=-3^{-\frac{1}{3}} x K_{2 / 3}(\xi) . "
\end{aligned}
$$


Summary details of tables of such Airy integrals (the first of their kind) now in proof, and of the mss. of fundamental tables from which these were prepared, are given in BAASMTC 8 , and in the following pages of this section. " $A(m)$ and $A i(x)$ are connected by the relation,

$$
A(m)=\left(\frac{2}{3} \pi^{2}\right)^{1 / 3} A i\left(-\pi^{2 / 3} m / 12^{1 / 3}\right) . "
$$

Airy's Integral is also of ten referred to under the form

If $x$ is positive,

$$
A_{1}(x)=\int_{0}^{\infty} \cos \left(w^{3}+x w\right) d w
$$

$$
A_{1}(x)=\pi 3^{-3 / 2} x^{\frac{1}{5}}\left[I_{-1 / 3}\left(3^{-\frac{1}{2}} \xi\right)-I_{1 / 3}\left(3^{-\frac{1}{\xi}} \xi\right)\right] .
$$

When the upper limit for $A(m)$ is $x$, the integral is denoted by $A(m, x)$ which was studied by both AIRY and RAYlEIGH.

Hardy generalized Airy's integral (see Watson 3, p. 320-324). If $T_{n}(t, \alpha)=t^{n} \cdot{ }_{2} F_{1}\left(-\frac{1}{2} n, \frac{1}{2}-\frac{1}{2} n ; 1-n ;-4 \alpha / t^{2}\right)$ one of the generalizations is the following:

$$
\begin{aligned}
C i_{n}(\alpha) & =\int_{0}^{\infty} \cos T_{n}(t, \alpha) d t \\
& =\left[2 \alpha^{1 / 2} \cos \left(\frac{1}{2} \pi / n\right) / n\right] K_{1 / n}\left(2 \alpha^{\frac{l n}{2 n}}\right), \text { or, } \\
C i_{n}(\alpha) & =\left[\pi \alpha^{\frac{1}{3}} / 2 n \sin \left(\frac{1}{2} \pi / n\right)\right]\left[I_{-1 / n}\left(2 \alpha^{\frac{1}{2 n}}\right)-I_{1 / n}\left(2 \alpha^{\frac{1}{2 n}}\right)\right] .
\end{aligned}
$$

When $n=3, T_{3}(t, \alpha)=t^{3}+3 \alpha t ; n=4, T_{4}(t, \alpha)=t^{4}+4 \alpha t^{2}+2 \alpha^{2}$; etc.

In this Section we shall need the special case of $n=1 / 3$ in the relation

$$
J_{n}(x)=u_{n}(x) \cos \left(x-\frac{1}{2} n \pi-\frac{1}{4} \pi\right)-v_{n}(x) \sin \left(x-\frac{1}{2} n \pi-\frac{1}{4} \pi\right),
$$

where $u_{n}, v_{n}$ are the integrals of SchafHeituIN (see Watson 1, p. 204-205).

It will be useful also to note the relations

$$
\begin{aligned}
& J_{-1 / 3}(x)=\left|H_{1 / 3}^{(1)}(x)\right| \cos \left[\frac{1}{3} \pi+\arg H_{1 / 3}^{(1)}(x)\right], \\
& Y_{-1 / 3}(x)=\left|H_{1 / 3}^{(1)}(x)\right| \sin \left[\frac{1}{6} \pi+\arg H_{1 / 3}^{(1)}(x)\right] .
\end{aligned}
$$

They offer the means of getting $J_{-1 / 3}(x)$ and $Y_{-1 / 3}(x)$ from WATson 3, T. 3, and conversely $H_{1 / 3}^{(1)}(x)$ from other tables of $J_{-1 / 3}(x)$ and $Y_{-1 / 3}(x)$.

As particular cases of the general formulae

$$
\begin{array}{r}
Y_{n}(x)=J_{n}(x) \cot n \pi-J_{-n}(x) \csc n \pi, \\
Y_{-n}(x)=J_{n}(x) \csc n \pi-J_{-n}(x) \cot n \pi,
\end{array}
$$

one may also note:

$$
Y_{-1 / 3}(x)=3^{-1}\left[2 J_{1 / 3}(x)-J_{-1 / 3}(x)\right], \quad Y_{1 / 3}(x)=3^{-1}\left[J_{1 / 3}(x)-2 J_{-1 / 3}(x)\right] .
$$

For zeros of AIRy's integrals $A i(x), A i^{\prime}(x), B i(x), B i^{\prime}(x)$, we shall use the notations $a_{s}, a_{s}{ }^{\prime}, b_{s}, b_{s}{ }^{\prime}$, respectively.

$$
A_{1} \cdot J_{ \pm 1 / 3}(x), J_{ \pm 2 / 3}(x), Y_{ \pm 1 / 3}(x), Y_{ \pm 2 / 3}(x)
$$

1. $13 \mathrm{D}, J_{ \pm 1 / 3}(x), J_{ \pm 2 / 3}(x)$, NYMTP $10, x=10(1) 25$

2. $10 \mathrm{D}, J_{ \pm 1 / 3}(x), J_{ \pm 2 / 3}(x)$, NYMTP $10, x=0(.01) 25$ 
3. 7D, $J_{1 / 8}(x), Y_{1 / 3}(x)$, WATSON $3, x=0(.02) 16$

4. 7D, $J_{-1 / 8}(x), Y_{-1 / 8}(x)$, Watson 3 , p. $715 \mathrm{f}, x=2(2) 16$

5. $5 \mathrm{D}, J_{ \pm 2 / 8}(x), Y_{ \pm 2 / 3}(x)$, KARAS, $x=0(.1) 10$

6. $5 \mathrm{D}, J_{ \pm 1 / 3}(x), Y_{ \pm 1 / 3}(x)$, HAYASHI $2, x=0(.1) 10$

7. 4-6S, $J_{ \pm 1 / 3}(x), J_{ \pm 2 / 3}(x)$, DiNNIK 14, p. 10 f, $x=0(.1) 15$; also, to $4 \mathrm{D}$, p. $26, x=.1$, $1(1) 5$

8. $4 \mathrm{D}, J_{1 / 3}(x), Y_{1 / 3}(x)$, GLAZENAP, $x=0(.02) 14(.04) 16$

9. $4 \mathrm{D}, J_{ \pm 1 / 8}(x), J_{ \pm 2 / 3}(x)$, GRISHKOV, $x=0(.1) 8$

10. $4 \mathrm{D}, J_{ \pm 1 / 8}(x)$, WATSON $1, x=0(.05) 2(.2) 8$

11. $4 \mathrm{D}, J_{ \pm 1 / 3}(x), J_{ \pm 2 / 3}(x)$, DinNIK $1,3,6, x=0(.2) 8$

$$
\text { A. } I_{ \pm 1 / 3}(x) \text { and } I_{ \pm 2 / 8}(x)
$$

1. 13S, NYMTP $10, x=10(1) 25$

2. $10 \mathrm{D}$, NYMTP $10, x=0(.01) 25$

3. 4-6ח, DinNiK 14, p. 13-15, $x=0(.1) 15$, except that in the cases of $I_{-1 / 3}(x), I_{-2 / 3}(x)$, $x \ngtr 6.4$; also to $4 \mathrm{D}$, p. $27, x=.1,1(1) 5$

4. 4-5S, GRISHKov, $x=0(.1) 8$

5. 4-5S, Dinnik 6, 9, 10 and JAhnke \& EMDe $1_{2}-1_{s}$ (p. 235), $x=0(.2) 8$

$$
\text { B. } e^{-x} I_{1 / 3}(x), e^{x} K_{1 / 3}(x),\left|H_{1 / 3}^{(1)}(x)\right|, \arg H_{1 / 3}^{(1)}(x), u_{1 / 3}(x), v_{1 / 3}(x)
$$

1. 7D, $\left|H_{1 / 3}^{(1)}(x)\right|$, arg $H_{1 / 3}^{(1)}(x), e^{x} K_{1 / 3}(x)$, WATSON $3, x=0(.02) 16$

2. $6 \mathrm{D}, e^{-x} I_{1 / 2}(x)$, BURSIAN, $x=1.2(.05) 4(.1) 16$

3. $4 \mathrm{D}, u_{1 / 3}(x),-v_{1 / 3}(x)$, WATSON $1, x=0(.05) 2(.2) 8$

\section{B. Other Functions}

1. $4 \mathrm{D}, n ! J_{n}(x), n= \pm 1 / 3, \pm 2 / 3$, DinNIK $2, x=0(.2) 8$

2. $4 \mathrm{~S}, \mp x^{3} J_{ \pm 1 / 3}\left(2 x^{8 / 2} / 3\right)$, GANS $1, x=0(.2) 2$

3. $3 \mathrm{~S}, \quad K=\tan \epsilon=\left[J_{2 / 3}(x)+J_{-2 / 3}(x)\right] /\left[J_{1 / 3}(x)-J_{-1 / 3}(x)\right]$, BREIT, $x=.2(.2) 2(.4) 5.2$, $K$ (to $3 \mathrm{~S}$ ), $2 \epsilon$ (to $4 \mathrm{~S}$ ), $\tan ^{-1} K$ (for $0^{\circ} .1$ )

4. $4 \mathrm{D},-v_{1 / 3}(x) / u_{1 / 3}(x)$, WATson $1, x=0(.05) 2(.2) 8$

5. 13D, first 15 derivatives of $J_{n}(x), I_{n}(x), n= \pm 1 / 3, \pm 2 / 3$, NYMTP $11, x=10(.1) 25$

6. $6 \mathrm{D},\left(\frac{1}{2} x\right)^{-1 / 8} I_{1 / 8}(x)$, BURSIAN, $x=0(.02) 1.2$

7. $4 \mathrm{D}, I_{2 / 3}(x) / I_{-2 / 3}(x)$, HeATLEY $1, x=0(.2) 6(.4) 8$

8. $4 \mathrm{~S}, 1-I_{1 / 3}(x) / I_{-1 / 3}(x)$, and its $\log$ to $4 \mathrm{D}, \Delta$, Heatley $1, x=0(.2) 5.2$

9. 3-4S, $\left[I_{-2 / 3}(x) / I_{2 / 3}(x)\right]-1$ and its $\log$, to $4 \mathrm{D}, \Delta$, Heatley $1, x=0(.2) 5.2$

10. 12D, first 18 derivatives of $x^{-n} J_{n}(x), x^{-n} I_{n}(x), n= \pm 1 / 3, \pm 2 / 3$, NYMTP 13 , $x=0(.1) 10$

$$
\text { C. Airy's integral } A(m)=\int_{0}^{\infty} \cos \frac{1}{2} \pi\left(w^{3}-m w\right) d w
$$

1. 5D, AIRY 3, $m=-4(.2)+4$; also, to $5 \mathrm{D}, A^{2}(m)$

2. $4 \mathrm{D}$, RAYLEIGH 3 and MASCART, p. $408, m=-4(.4)+4$

3. 5D, AIRY 5, and HogNER with graph, $m=-5.6(.2)+5.6$

4. 5D, MAscarT, p. 397 , also, to $4 \mathrm{D}, A^{2}(m), m=-5.6,-5(1)-1,-.2(.2)+5.6$

5. 3D, $A^{2}(m)$, Pernter, p. $517, m=-2(.2)+13.6$

6. $5 \mathrm{D},\left(3 / \pi^{2}\right)^{1 / 3} A(m)$, Nicholson $1, m=-4(.2)+4$

7. 7D, $A(m, x)=\int_{0}^{x} \cos \frac{1}{2} \pi\left(w^{3}-m w\right) d w, m=-4(.4)+4$, AIRY $3, x=1,1.26,1.44$, $1.58,1.70,1.92,2$

8. 4D, $A(m, x)=\int_{0}^{x} \cos \frac{1}{2} \pi\left(w^{3}-m w\right) d w, m=-4(.4)+4$, RAYLEIGH 3, and MASCART, p. $408, x=1,1.26,1.44, \infty$ 


$$
\begin{gathered}
\mathrm{C}_{2 .} \text { Airy integrals } A i(x)=(1 / \pi) \int_{0}^{\infty} \cos \left(\frac{1}{3} t^{3}+x t\right) d t, \\
B i(x)=(1 / \pi) \int_{0}^{\infty}\left[e^{\left(-i t^{3}+x t\right)}+\sin \left(\frac{1}{3} t^{3}+x t\right)\right] d t
\end{gathered}
$$

1. 12D, $A i(x)$, J. C. P. Miller, $x=-20(.05)-10(.1) 0$ and to $12 \mathrm{~S}, x=0(.1) 10$ (21D at $x=10)$

2. $12 \mathrm{D}, B i(x)$, J. C. P. MILLER, $x=-10(.1) 2.8$

3. $8 \mathrm{D}, A i(x), A i^{\prime} x$, J. C. P. MiLleR, $x=-20(.01)+2, \delta_{m}^{2}$

4. $8 \mathrm{D}, B i(x)$, J. C. P. MILLER, $x=-10(.1)+2.5$

5. $8 \mathrm{D}, \log A i(x), \log B i(x)$, J. C. P. MILLER, $x=0(.1) 10, \delta_{m}^{2}$

6. 7D, $A i^{\prime}(x) / A i(x), B i^{\prime}(x) / B i(x)$, J. C. P. MILLER, $x=0(.1) 10, \delta_{m}{ }^{2}$

7. 8D, 7D, $F(x), G(x)$, J. C. P. MilleR, $x=[-80(1)-30(.1) 0 ; 8 \mathrm{D}],[0(.1) 2.5 ; 7 \mathrm{D}], \delta_{m}^{2}$

8. $6 \mathrm{D}$ of $1^{\circ}, x(x), \psi(x), \mathrm{J}$. C. P. MILLER, $x=-80(1)-30(.1)+2.5, \delta_{m}^{2}$

\section{C.. Solutions of $S^{\prime \prime}=9 x S$}

1. 3-5S, 2 solutions of this equation, $S_{1}=s_{1}+i t_{1}, S_{2}=s_{2}+i t_{2}$, are given by RAYLEIGH 8 for $\eta=0(.1) 2.5$, where $x=i \eta$. "These may be related to $A i\left(3^{2 / 3} i x\right)$ and $B i\left(3^{2 / 8} i x\right)$ " J. C. P. Miller. Rayleigh gives also a table, for $\eta=[.1(.2) 2.5 ; 3-4 \mathrm{~S}]$, of $s_{1}^{2}-t_{1}^{2}$, $\left(s_{1}^{2}+t_{1}^{2}\right)^{2},\left(s_{1}^{2}-t_{1}^{2}\right) /\left(s_{1}^{2}+t_{1}^{2}\right)^{2}$.

\section{Other related Airy integrals}

1. $4 \mathrm{D},(1 / a) \int_{0}^{a} \exp \left[\frac{1}{2} i \pi\left(u^{3}+2 u x / a\right)\right] d u$, STEWARD, p. $148, x=0(1) 2, a=2^{1 / 3}$

2. $4 \mathrm{D}, \int_{0}^{1} \cos \frac{1}{2} \pi\left[u^{3}+(2 n-3) u\right] d u$, STEWARD, p. 149 , for $n=0,1,5 / 4,3 / 2,2$

3. $4 \mathrm{D}, 1 / a \int_{0}^{1} \cos \frac{1}{2} \pi\left[u^{3}+(n-3) a^{2} u\right] d u$, STEWARD, p. 149 for $n=2,2 \frac{1}{2}, 2 \frac{1}{2}, 3$

4. $3 \mathrm{D}, \omega(x)=\pi^{-\mathrm{j}} \int_{0}^{\infty} \cos \left(\frac{1}{3} t^{3}-x t\right) d t$, HELD, $x=-9,-5,-4,-3.5(.5)-2.5$, $-2.4(.1)+2.4$. Also, to $2 \mathrm{D}, \int_{-\infty}^{2.3} \omega^{2}(x) d x$

5. 4-5D, $\int_{-\infty}^{-i \infty} e^{i\left(x t^{2}+t^{8}\right)} d t$, HoGNER, real and imaginary parts for $x=-3.2(.2)+3.2$.

There is a graph of the integra! for this range.

\section{Zeros}

1. 15D, $J_{-1 / 3}(x)$, LEHMER 2 , first zero

2. $10 \mathrm{D}, J_{ \pm 1 / 8}(x), J_{ \pm 2 / 3}(x)$, NYMTP 12 , first 8 zeros

3. 9D, $J_{-1 / 3}(x)$, IKEDA, first zero

4. 7D, $J_{1 / 3}(x), Y_{1 / 3}(x), J_{-1 / 3}(x) \pm J_{+1 / 8}(x)$, WATSON 3, first 40 zeros

5. 7D, $J_{2 / 3}(x)$, EMDE 1 , first zero

6. 6D, $J_{1 / 3}\left(2 / 3 x^{3 / 2}\right)+J_{-1 / 3}\left(2 / 3 x^{3 / 2}\right)$, J. M. BATES, first 5 zeros

7. $4 \mathrm{D}, J_{1 / 3}(x), Y_{1 / 3}(x)$, GLAZENAP, first 40 zeros

8. $4 \mathrm{D}, J_{ \pm 1 / 3}(x)$, ArRey 18 and Gray \& Mathews $1_{2}$, p. 317 , first zeros

9. $3 \mathrm{D}, J_{ \pm 1 / 3}(x), J_{ \pm 2 / 3}(x)$, DiNNIK 14 , p. 12 , first 5 zeros

10. 2D, $J_{ \pm 1 / 3}(x), J_{ \pm 2 / 3}(x)$, DinNIK 3,6 , first 3 zeros; also DinNIK 1, first 2 zeros, and 3 zeros for $J_{-2 / 8}(x)$

11. 2D, $J_{n}(x)$, DinNIK 2 and AIREY 18, $n=1 / 3,2 / 3$, first 2 zeros; $n=-1 / 3,-2 / 3$, first 3 zeros

12. $2 \mathrm{D}, J_{ \pm 1 / 3}(x), J_{ \pm 2 / 3}(x)$, DiNNIK 5 , first zeros

13. 3-4S, $J_{-2 / 3}(x)-J_{2 / 3}(x)$, MACDONALD, first 3 zeros

14. $4 \mathrm{D}, A(m)$, STOKEs 1 , first 50 zeros, $\Delta$

15. $4 \mathrm{D}, A^{\prime}(m)$, STOKEs 1 , first 10 zeros, $\Delta$

16. 8D, $a_{s}, a_{s^{\prime}}, A i^{\prime}\left(a_{s}\right), A i\left(a_{s}^{\prime}\right)$, J. C. P. MILLER, $s=1(1) 50$

17. $8 \mathrm{D}, b_{\triangleleft}, b_{\iota^{\prime}}, B i^{\prime}\left(b_{s}\right), B i\left(b_{\bullet}{ }^{\prime}\right)$, J. C. P. MILleR, $s=1(1) 20$ 


\section{E. Graphs}

1. $J_{-1 / 3}(x) / J_{1 / 3}(x), J_{+2 / 3}(x) / J_{-2 / 3}(x)$, BoRGNIS, $x=3 i, 2 i, i, 0(1) 9$

2. $J_{-1 / 3}(x)+J_{1 / 3}(x)$, BORGNIS, $0<x<2.4$

3. $J_{-1 / 3}(x)+J_{+1 / 3}(x), \sqrt{3}\left[J_{-1 / 3}(x)-J_{+1 / 3}(x)\right]$, JAHNKE \& EMDE $1_{2}$ (p. 209) - $1_{5}$ (p. 142)

4. $\left[J_{-2 / 3}(x)-J_{+2 / 3}(x)\right] /\left[J_{1 / 3}(x)+J_{-1 / 3}(x)\right]$, BORGNIS, $0<x<2.6$

5. $I_{1 / 3}(x), I_{2 / 3}(x)$, JAHNKE \& EMDE $1_{2}$ (p. 285) - $1_{5}$ (p. 235)

6. $x$. $\left[I_{-2 / 3}(x)-I_{+2 / 3}(x)\right] /\left[I_{-1 / 3}(x)-I_{+1 / 3}(x)\right]$, BoRGNIS, $0<x<3.2$

7. $(2 \sqrt{3} / \pi) K_{n}(x), 0<x<24$, and their derivatives, $0<x<6, n=1 / 3,2 / 3$, KRAmERS

8. $x K_{1 / 3}(x), x K_{2 / 3}(x)$, KRAMERS, $0<x<24$

9. $\left(4 x^{2} / \pi^{2}\right)\left[K_{1 / 3}^{2}(x)+K_{2 / 3}^{2}(x)\right]$ and its derivative, KRAMERS

10. $H_{ \pm 1 / 3}^{(1)}(i x)$, JAHNKe \& EMDE $1_{2}$ (p. 208) - $1_{6}$ (p. 141), $0<x<15$

11. $i^{1 / 3} H_{1 / 3}^{(1)}(i x)$, JAHNKE \& EMDE $1_{2}$ (p. 285) $-1_{5}$ (p. 235), $0<x<8$

12. $[A(m)]^{2}$, Pernter $1_{2}$, p. 430 , and Humphreys $1_{4}$, p. 492

13. $\sqrt{I}=\int_{0}^{1} \cos \left(4 u^{3}-z u\right) d u$, STEWARD, p. 184

See also $\mathrm{VC}_{1} 3$

$$
\text { VI. } J_{n}(x), n= \pm \frac{1}{4}, \pm \frac{3}{4}, \pm \frac{1}{6}, \pm \frac{5}{6} \text {, ETC. }
$$

The functions $F(x)=\int_{-\infty}^{\infty} \cos \left(t^{4}+x t\right) d t, \quad G(x)=\int_{-\infty}^{\infty} \sin \left(t^{4}+x t\right) d t$ were studied by Rayleigh 2, particularly in the case $x=0$. J. Fischer continued the work and plotted the quantity $F^{2}+G^{2}$. He also discussed some integrals which were shown by G. H. HARDY ${ }^{1}$ to be expressible in terms of Bessel functions. If $8 u=x^{2}+\pi, 8 v=x^{2}-\pi, M=(\pi / 8)(2 x)^{\frac{1}{2}}, x>0$,

$$
\begin{aligned}
K(x) & =\int_{0}^{\infty} \cos \left(t^{4}-x t^{2}\right) d t=M\left[\cos u J_{1 / 4}\left(x^{2} / 8\right)+\cos v J_{-1 / 4}\left(x^{2} / 8\right)\right], \\
K(-x) & =M\left[\cos u J_{-1 / 4}\left(x^{2} / 8\right)-\cos v J_{1 / 4}\left(x^{2} / 8\right)\right], \\
L(x) & =\int_{0}^{\infty} \sin \left(t^{4}-x t^{2}\right) d t=-M\left[\sin u J_{1 / 4}\left(x^{2} / 8\right)+\sin v J_{-1 / 4}\left(x^{2} / 8\right)\right], \\
L(-x) & =M\left[\sin u J_{1 / 4}\left(x^{2} / 8\right)-\sin v J_{-1 / 4}\left(x^{2} / 8\right)\right], \\
E(x) & =\int_{0}^{\infty} e^{-t^{4}-x t^{2}} d t=1 / 4 x^{\frac{1}{2}} e^{x^{2} / 8} K_{1 / 4}\left(x^{2} / 8\right) .
\end{aligned}
$$

The integrals $E, F, G, K, L$ are related to integrals of O'Toole and MASCART $\int_{a}^{b} e^{i\left(t^{4}+z t^{2}-w t\right)} t d t$, which occur in a problem relating to circular diaphragm.

HeAtley 2 has defined the Toronto function $T(m, n, x)$ as

$$
\int_{0}^{\infty} t^{m-n} e^{-p^{2} t^{2}} I_{n}(2 a t) d t
$$

where $x=a / p$. In a table of $T(m, n, x)$ for different values of $m$ and $n$, expressed in terms of other functions, there are 10 in terms of the function $I_{n}\left(\frac{1}{2} x^{2}\right)$; one of 5 of these involving $I_{ \pm 1 / 4}\left(\frac{1}{2} x^{2}\right)$ and $I_{ \pm 3 / 4}\left(\frac{1}{2} x^{2}\right)$, is $T\left(-\frac{1}{2}, \frac{1}{2}, x\right)$ $=2 x^{3} \pi^{\frac{1}{3}} e^{-\frac{1}{2} x^{2}}\left[I_{-1 / 4}\left(\frac{1}{2} x^{\frac{1}{3}}\right)-I_{3 / 4}\left(\frac{1}{2} x^{\frac{1}{3}}\right)\right]$.

1 G. H. HARDy, "On certain definite integrals considered by Airy and Stokes," Quart. J. Math., v. 41, 1910, p. 226-240. 


$$
\text { A. } J_{n}(x), Y_{n}(x)
$$

1. $13 \mathrm{D}, J_{n}(x), n= \pm 1 / 4, \pm 3 / 4$, NYMTP $10, x=10(1) 25$

2. 13D, first 15 derivatives of $J_{n}(x), n= \pm 1 / 4, \pm 3 / 4$, NYMTP $11, x=10(.1) 25$

3. 12D, first 18 derivatives of $x^{-n} J_{n}(x), n= \pm 1 / 4, \pm 3 / 4$, NYMTP $13, x=0(.1) 10$

4. $10 \mathrm{D}, J_{n}(x), n= \pm 1 / 4, \pm 3 / 4$, NYMTP $10, x=0(.01) 25$. Also $u_{n}(x)$ and $v_{n}(x)$, to $10 \mathrm{D}, 25 \leq x<30000$, various intervals, in the asymptotic expansion of these functions

5. $10 \mathrm{D}, J_{ \pm 1 / 4}(x)$, AIREY $33, x=5$

6. 7D, $J_{n}(x), n= \pm 1 / 4, \pm 3 / 4$, BAASMTC $7, x=0(.1) 20$

7. $6 \mathrm{D}, J_{n}(n), J_{n-1}(n),-Y_{n}(n),-Y_{n-1}(n), n=0(1 / 4) 10$, AIREY 20

8. $6 \mathrm{D}, J_{n}(x), n-x=-1(.1)+1$, AIREY $31, x=1(1) 20$

9. $4 \mathrm{D}, J_{n}(x), n= \pm 1 / 4, \pm 3 / 4$, DinNik $5,8,10, x=0(.2) 8$

10. $4 \mathrm{D}, J_{n}(x), n= \pm 1 / 4, \pm 3 / 4, \pm 1 / 6, \pm 5 / 6$, DINNIK 14, p. $16-18,21-23, x=0(.1) 15$

11. $4 \mathrm{D}, J_{ \pm 1 / 8}(x)$, DiNNIK $6, x=0(.2) 8$

12. $4 \mathrm{D}, J_{n}(x), Y_{n}(x), n= \pm 1 / 4, \pm 3 / 4$, KARAS, $x=0(.1) 8$

13. 3D, $D(x)=2^{-1}(-1 / 4) ! x^{1 / 4} J_{-1 / 4}\left(\frac{1}{2} x\right), G(x)=-2^{3}(1 / 4) ! x^{-1 / 4} J_{1 / 4}\left(\frac{1}{2} x\right)$, PRANDTL and JAHNKE \& EMDE $1_{1}$, p. $106, x=0(.2) 8(1) 12$

$$
\text { A } . I_{n}(x)
$$

1. $13 \mathrm{D}, n= \pm 1 / 4, \pm 3 / 4$, NYMTP $10, x=10(1) 25$

2. 13D, first 15 derivatives of $I_{n}(x), n= \pm \frac{1}{4}, \pm \frac{3}{4}$, NYMTP $11, x=10(.1) 25$

3. 12D, first 18 derivatives of $x^{-n} I_{n}(x), n= \pm \frac{1}{4}, \pm \frac{3}{4}$, NYMTP $13, x=0(.1) 10$

3A. 10D, $n= \pm \frac{1}{4}, \pm \frac{3}{4}$, NYMTP $10, x=0(.01) 25, \delta_{m}^{2 n}$

4. 5D, $T(m, n, x)$ for $m= \pm \frac{1}{2}, n= \pm \frac{1}{2}, \pm \frac{3}{2}$, HeAtLEY 2 , p. 26,28 , $x=0(.2) 4(1) 6,10,25,50 ; T(m, n, x)$ may here be expressed as functions of two or more of the functions $I_{n}(x), n= \pm 1 / 4, \pm 3 / 4$

5. 4-5D, $x(x)=\int_{0}^{\infty} e^{-\left(x-w^{2}\right)^{2}} d w=\frac{1}{2} \pi x^{\frac{1}{3}} e^{-3 x^{2}}\left\{I_{-1 / 4}\left(\frac{1}{2} x^{2}\right)+I_{1 / 4}\left(\frac{1}{2} x^{2}\right)\right\}, x>0$, HARTREE \& Johnston, $\left.-x=[0(.1) 2.4 ; 4 \mathrm{D}], \Delta^{2} ;-x=[2.5(.1) 3.2 ; 5 \mathrm{D}], \Delta^{2} ; x=[0(.1) 3) ; 4 \mathrm{D}\right], \Delta^{2}$; $[x=2.8(.2) 8 ; 4 \mathrm{D}], \Delta$. Graph. $\left[x(-x)=\frac{1}{2} \pi x^{\frac{1}{3}} e^{-3 x^{2}}\left\{I_{-1 / 4}\left(\frac{1}{2} x^{2}\right)-I_{1 / 4}\left(\frac{1}{2} x^{2}\right)\right\}=\frac{1}{2}(2 x)^{3} e^{-3 x^{2}} K_{1 / 4}\left(\frac{1}{2} x^{2}\right)\right]$

6. 4-6S, $I_{n}(x), n=1 / 4,3 / 4,1 / 6,5 / 6$, DiNNIK 14, p. 18f, 24f, $x=0(.1) 15$

7. 4-5S, $n=-1 / 4$, DiNNiK 14 , p. 18f, $x=0(.1) 8.1$

8. $5 \mathrm{~S}, n=-3 / 4$, Dinnik 14 , p. $18 \mathrm{f}, x=0(.1) 8$

9. $4-5 S, n= \pm 1 / 4, \pm 3 / 4$, DINNIK 10 , p. $132, x=0(.2) 8$

10. $5 \mathrm{~S}, n=-1 / 6,-5 / 6$, DiNNIK 14 , p. $24, x=0(.1) 5.5$

\section{B. Zeros}

1. 10D, $J_{ \pm 1 / 4}(x), J_{ \pm z / 4}(x)$, NYMTP 12 , first 8 zeros

2. 5D, $J_{n}(x), n=-1(.01)+1$, AIREY 28 , first zeros

3. 5S, $J_{-1 / 4}\left(\frac{1}{2} x^{3}\right)$, Michell 1 , first 2 zeros [16.101, 104.98]

4. $4 \mathrm{~S}, J_{-1 / 4}\left(\frac{1}{2} x^{3}\right)$, PRESCOTT, p. 510-511, first zero

5. $4 \mathrm{~S}, J_{-3 / 4}\left(\frac{1}{2} x^{4}\right), J_{-1 / 6}(x)$, Michell 1 , first zeros [4.4817, 41.035]

6. $4 \mathrm{D}, J_{n}(x), 40 n=-20(1)+40$, AIREY 18, and GRAY $\&$ MATHEWs $1_{2}$, first 2 zeros; also first zeros for $\pm 6 n=1$

7. $4 \mathrm{D}, j_{n, 1}^{\prime} /(2 n)^{-i}, j_{n, 2}^{\prime}, 40 n=-20(1)+20$, AIREY 18; also $j_{n, 2}^{\prime}$ to $5 \mathrm{D}, 4 n=-1$

8. $4 \mathrm{D},\left(j_{n, 1}^{\prime}\right)^{2} / 4(n+1), 40 n=-40(1) 0$, AIREY 18

9. $4 \mathrm{D}, J_{ \pm 1 / 6}(x)$, Airey 18 , and Gray \& Mathews $1_{2}$, first zero

10. $4 \mathrm{D}, J_{n}(x), n=-.4(.01)+1.49, \Delta$, JAHNKE \& EMDE $1_{2}$ (p. 238) $-1_{5}$ (p. 167), first zeros, also first 2 zeros $n=-.4(.01)+.99, \Delta$

11. 3D, $J_{-5 / 4}(x), J_{-7 / 4}(x)$, AIREy 15 , first imaginary zeros

12. $3 \mathrm{D}, J_{-3 / 7}(x)$, ONo, first zero

13. 3D, $J_{n}(x), n=0(.1) 1$, EMDE 1 , first zeros; also first zeros, exact or to $6 \mathrm{D}, n=-1\left(\frac{1}{2}\right)+2$

14. 3D, $J_{ \pm 1 / 4}, J_{ \pm z / 4}$, HidAKA, first 4 zeros

15. $3 \mathrm{D}, J_{ \pm 1 / 4}(x), J_{ \pm 3 / 4}(x), J_{ \pm 1 / 6}(x), J_{ \pm 5 / 6}(x)$, DinNIK 14 , first 5 zeros 
16. 2D, $J_{-1 / 4}(x), J_{-3 / 4}(x)$, Dinnik 8, 10, and Airey 18, first 3 zeros; also first 2 zeros of $J_{+1 / 4}(x), J_{+3 / 4}(x)$

17. 2D, $J_{ \pm 1 / 6}(x)$, DinNIK 6, first 3 zeros

18. $2 \mathrm{D}, J_{ \pm 1 / 4}(x), J_{ \pm z / 4}(x)$, DinNIK 5 , first zeros; also first zeros, to $2 \mathrm{D}$, of $J_{ \pm 1 / 6}(x)$

19. $2 \mathrm{D}, J_{1 / 4}(x) J_{-1 / 4}(k x)-J_{-1 / 4}(x) J_{1 / 4}(k x)$ and $J_{3 / 4}(x) J_{1 / 4}(k x)+J_{-3 / 4}(x) J_{-1 / 4}(k x)$, DiNNIK 10 , p. $19 \mathrm{f}$, first zeros, $k=0(.1) 1$, and $k=0(.2) 1$, respectively

\section{Graphs}

1. $J_{ \pm 3 / 4}(x), J_{ \pm 5 / 4}(x)$, RUEDy $3,0<x<8$

2. $F^{2}(x)+G^{2}(x)$, J. FisCHER 1, p. $389, y / \lambda=-6(2)+6$; also $K^{2}(x)+L^{2}(x)$, p. 393, $-100<x / \lambda<+110$, where $10 x=2 \pi\left(2^{3 / 4}\right)(y / \lambda)$

3. $\sigma_{m}(x)=\sum_{n=1}^{\infty} n^{-m}\left[2.06\left\{3 \pi^{3} / 2^{-11 / 6}\right\}(2 / 3) ! n^{5 / 6} x^{-1 / 6} J_{1 / 6}(n x)-.64 x^{-1} \sin n x\right], m=2,3$, W. W. HANSEN, $0<x<3.2$

4. $\left(1+x^{2} / \rho^{2}\right)^{-1 / 8} K_{1 / 4}\left(2 \pi \rho^{2}\left[1+x^{2} / \rho^{2}\right]^{3}\right) / K_{1 / 4}\left(2 \pi \rho^{2}\right)$, CAMpbell $\&$ FosTeR, $\rho=0, .01, .1$, $.5, \infty,-1<x<+1$

See also VI $\mathrm{A}_{2} 5$

\section{BESSEL-Clifford Functions $x^{m} J_{n}\left(x^{s}\right)$}

ClIFFORD recommended ${ }^{1}$ the use of the functions defined by the equations

(1) $C l_{0}(x)=J_{0}\left(2 x^{3}\right)$,

$C l_{n}(x)=(-1)^{n} d^{n} C l_{0}(x) / d x^{n}=x^{-\frac{1 n}{n}} J_{n}\left(2 x^{\frac{1}{2}}\right)$.

The more important properties and the applications of these functions are set out in detail by Greenhill. ${ }^{2}$ AIRey constructed tables of $\mathrm{Cl}_{0}(x)$ and $\mathrm{Cl}_{1}(x)$. Series involving the functions $\mathrm{Cl}_{n}(-x)$ occur in work of ScHumanN on the heating of a porous body by a warm fluid. He gives (p. 412-413) graphs of

$$
T_{s}=e^{-y-z} \sum_{n=1}^{\infty} z^{n} C l_{n}(-y z), \quad T_{L}=e^{-y-z} \sum_{n=0}^{\infty} z^{n} C l_{n}(-y z),
$$

for $y=0(1) 10, z=0(1) 10$. These series represent the temperature in the solid and liquid respectively, the units being chosen so that $T_{8}+T_{L}=1$, when $y=z$.

In I (3) we had $\Lambda_{n}(x)=n !\left(\frac{1}{2} x\right)^{-n} J_{n}(x)$, and in $\mathbf{I A}_{2}$ are a number of functions involving $\Lambda_{n}(x)$ for the case when $n=1$.

In his Institutionum Calculi Integralis, v. 2, St. Petersburg, 1769, p. $187-189$, EULER gave the complete solution of $x y^{\prime \prime}+a y=0$ in the form of series equivalent to $x^{\frac{1}{2}} J_{1}\left(4 a^{\frac{1}{2}} x^{\frac{1}{2}}\right)$ and $x^{\frac{1}{2}} Y_{1}\left(2 a^{\frac{1}{3}} x^{\frac{1}{3}}\right)$.

In the paper of Michelson \& PEASE on the measurement of the diameter of $\alpha$-Orionis, use was made of the following integral for which Moulton prepared a table and graphs:

$$
F(x, n)=\int_{0}^{1}\left(1-t^{2}\right)^{n+\frac{1}{3}} \cos (x t) d t=\left(\pi^{\frac{3}{3}} / 2\right) n !(2 / x)^{n+1} J_{n+1}(x) .
$$

Hamy published tables of

$$
S\left(\frac{1}{2} x\right)=(4 / \pi) F(x, 0), \quad \text { and } \quad T\left(\frac{1}{2} x\right)=(4 / 3 \pi) F(x, 1) .
$$

1 W. K. Clifford, Mathematical Papers, London, 1882, p. 346-349.

2 G. Greenhill, Phil. Mag., s. 6, v. 38, 1919, p. 501-528. 


\section{A. Tables and Graphs}

1. $6 \mathrm{D}, C l_{0}(x), C l_{1}(x)$, AIREy $22, x=0(.02) 20$

2. $3 \mathrm{D}, x^{3} J_{3}\left(2 x^{3}\right), x^{3} Y_{3}\left(2 x^{3}\right)$, BATES \& MASSEY $x=1(.1) 2(.25) 5$

3. $C l_{0}(x), C l_{1}(x)$, JAHNKE \& EMDE $1_{2}$ (fig. 122) - $1_{6}$ (fig. 89), graphs, $0<x<60$

4. $9 \mathrm{D}, \Lambda_{n}(x)$, NYMTP $4, n=2,3, x=0(.05) 10 ; n=4,5, x=0(.05) 6.5(.1) 10 ; n=6,7,8$, $x=0(.05) 1.5(.1) 10 ; n=9(1) 20, x=0(.1) 10 ; \delta_{m}^{2}$

5. 7D, $\Lambda_{n}(x)$, DARMSTADT

6. $4 \mathrm{D}, \Lambda_{n}(x)$, JAHNKE $\&$ EMDE $1_{2}$ (p. 250f) $-1_{5}$ (p. 180f), $n=0(1) 8 ; x=0(.02) 10$. Also graphs, $1_{2}$ (fig. 132) $-1_{5}$ (fig. 99), $0<x<10$

7. 3D, $F(x, n)$, Moulton, $n=0,1,2 ; x$ varying differently for different values of $n$, e.g.: $n=1, x=0,100^{\circ}, 130^{\circ}, 160^{\circ}, 200^{\circ}, 230^{\circ}, 240^{\circ}\left(40^{\circ}\right) 440^{\circ}, 520^{\circ}, 600^{\circ} ; n=2$, $x=0\left(40^{\circ}\right) 720^{\circ}$

8. $4 \mathrm{D},(4 / \pi) F(x, 0),(4 / 3 \pi) F(x, 1)$, HAMY, for $x=0(.2) 4.2$

9. $6 \mathrm{~S}, n !\left(-\frac{1}{2} x\right)^{-n} e^{x} I_{n}(x)$, AIREY 27, p. $231,235,239,241, n=0\left(\frac{1}{2}\right) 1 \frac{1}{3}$, $x=0(.01) .05(.025) .5(.05) 1(.1) 1.5(.25) 4$; see III A2 33

See also I $\mathrm{A}_{2}$

\section{VIII. $J_{n}(x)$, AND OTHER FUNCTIONS, AS Functions OF THE ORDER $n$}

The function

$$
\bar{D}_{n}(x)=\partial J_{n}(x) / \partial n
$$

first became important when STOKEs, following the method of PoIsson, obtained a second solution of Bessel's equation of zero order by considering the limit of the quantity

$$
(1 / n)\left[J_{n}(x)-(-1)^{n} J_{-n}(x)\right]
$$

as $n$ tends to zero. A similar device was employed later by other writers in obtaining a second solution of Bessel's equation when the order is an integer.

SchAFHEITLIN found a remarkable connection between the function $\bar{D}_{n}(x)$ and the functions $S i(x), C i(x)$ [see Section IV where $V_{n}(x) \equiv \bar{D}_{n}(x)$ ]. His relations

$$
\begin{aligned}
\bar{D}_{i}(x) & =J_{i}(x) \operatorname{Ci}(2 x)-J_{-i}(x) \operatorname{Si}(2 x), \\
\bar{D}_{-i}(x) & =J_{-i}(x) \operatorname{Ci}(2 x)+J_{i}(x) \operatorname{Si}(2 x),
\end{aligned}
$$

were rediscovered by ANSELL \& FISHER ${ }^{1}$ soon after Fisher had shown the importance of $\bar{D}_{n}(x)$ in the theory of statistical estimations.

AIREY 30 has constructed tables of $\bar{D}_{ \pm \xi}(x)$ and $\bar{D}_{ \pm 3 / 2}(x)$ and has shown that values of $\bar{D}_{n}(x)$ when $n$ is half an odd integer may be derived by means of the recurrence relation

$$
\bar{D}_{n-1}(x)+\bar{D}_{n+1}(x)=(2 n / x) \bar{D}_{n}(x)+(2 / x) J_{n}(x) .
$$

For integral values of $x$ he gives the relations

$$
\begin{aligned}
& \bar{D}_{n}(x)=-G_{n}(x)+\frac{1}{2}(n !) \sum_{s=0}^{n-1}(2 / x)^{n-s} J_{s}(x) / s !(n-s) ! \\
& \bar{D}_{-n}(x)=(-1)^{n+1}\left[G_{n}(x)+\frac{1}{2}(n !) \sum_{s=0}^{n-1}(2 / x)^{n-s} J_{s}(x) /(s !(n-s) !)\right] .
\end{aligned}
$$

1 P. R. Ansell \& R. A. Fisher, "Note on the numerical calculation of a Bessel function derivative," London Math. So., Proc., s. 2, v. 24, Records, June 11, 1925, p. iii-v. 


\section{A. Tables, graphs, and charts}

1. $6 \mathrm{D}, \bar{D}_{n}(x), n= \pm .5, \pm 1.5$, AIREY $30, x=0(.1) 20$

2. $5 \mathrm{D}, \bar{D}_{n}(x), n-x=-1(.1)+1$, AIREY $32, x=0(.1) 20$

3. $4 \mathrm{D}, \partial \bar{D}_{n}(x) / \partial n, n-x=-1(.1)+1$, AIREY $32, x=0(.1) 20$

4. $\bar{D}_{n}(x), n= \pm .5, \pm 1.5$, graphs, JAHNKE \& EMDE $1_{2}$ (fig. 96) $-1_{5}$ (fig. 71 ), $0<x<20$

5. First function values $J_{n}(x)=.001, J_{n}(x)=.005$, graphs, JAHNKE \& EMDE $1_{2}$ (fig. 127) $-1_{5}$ (fig. 94), $10<n<5000$

6. $J_{n}(x)$ as a function of $x$ and $n$, relief, JAHNKE \& EMDE $1_{2}$ (figs. 92-93) - $1_{5}$ (figs. 67-58), (67) $n=0$ (1) $10, x=0$ (1) 20 ; (68) $n=-4$ (1) $0, x=0$ (1) 14

7. Curves $J_{n}(x)=$ const. in the plane $n, x$, JAHNKE \& EMdE $1_{2}$ (fig. 114) - $1_{5}$ (fig. 84), $n=-4(1)+8, x=0(1) 18$

8. Curves of no. 7, shown on the surface $z=J_{n}(x)$, JAHNKE \& EMDE $1_{2}$ (fig. 115) $-1_{6}$ (fig. 85)

9. $j_{n, 8}-n$ as a function of $n$, graph, JAHNKe \& EMDE $1_{8}-1_{5}$ (figs. $a 87, b 88, c 90$ ), (a) $n=0(1) 20$; (b) $n=20(5) 100$; (c) $n=50(50) 1000$

10. Curves $Y_{n}(x)=$ const., JahnKe \& EMde $1_{2}$ (fig. 136) $-1_{5}$ (fig. 103), $n=-5(1)+6$, $x=0(1) 16$

11. Surface $z=I_{n}(x)$, JAHNKE \& EMDE $1_{3}-1_{5}$ (fig. 126), $n=0(2) 10, x=0(2) 10$

See also: I C 1 1, 2, 4-8, 14-19, 22, 24-25, 27-30; I C 1 ; IE, 7-16; II A A $_{2}$-9; III A 1-2, 4-13; etc.

IX. The Functions of Lommel $\left[s_{m, n}(x), S_{m, n}(x)\right]$, SCHLÄFLI $\left[F_{n}(x)\right]$, LOMMEL-WEBER $\left[\Omega_{n}(x)\right]$, RAYLEIGH $\left[r_{1}(x)\right]$, AND Struve $\left[H_{n}(x)\right]$

The functions $s_{m, n}(x), S_{m, n}(x)$ of LommeL ${ }^{1}$ may be defined by the equations

$$
4 a c s_{m, n}(x)=x^{m+1} F\left(1 ; a+1, c+1 ;-\frac{1}{4} x^{2}\right)
$$

$S_{m, n}(x)=s_{m, n}(x)+2^{m-1}(a-1) !(c-1) ! \csc (n \pi)\left[\sin (a \pi) J_{-n}(x)-\sin (c \pi) J_{n}(x)\right]$ where $2 a=m-n+1,2 \mathrm{c}=m+n+1$.

The related function $T_{1, n}(x)$ of GoldsteIN is given by

$$
T_{1, n}(x)=S_{1, n}(i x)-n \exp \left(\frac{1}{2} n \pi i\right) K_{n}(x) .
$$

The function $H_{n}(x)$, which has been called Struve's function (STRUve $1,2)$ is related to $S_{m, n}(x)$ in the following way

If

$$
H_{n}(x)=Y_{n}(x)+2^{1-n} S_{m, n}(x) /\left[\frac{1}{2} \pi^{\frac{1}{3}}\left(n-\frac{1}{2}\right) !\right] .
$$

$$
R(x)=\frac{1}{1^{2} \cdot 3}-\frac{x^{2}}{1^{2} \cdot 3^{2} \cdot 5}+\frac{x^{4}}{1^{2} \cdot 3^{2} \cdot 5^{2} \cdot 7}-\cdots=\left(\frac{1}{2} \pi / x^{2}\right) H_{1}(x),
$$

the function $r_{1}(x)$ used by RAYLEIGH 2 is $r_{1}(x)=\left(2 x^{3} / \pi\right) R(x)$ while generally

$$
H_{n}(z)=\left[2\left(\frac{1}{2} z\right)^{n} \pi^{-\frac{1}{2}} /\left(n-\frac{1}{2}\right) !\right] \int_{0}^{1} \sin (z u)\left(1-u^{2}\right)^{n-\frac{1}{2}} d u .
$$

The integral tabulated in STRuve 2 may be expressed in the form $\int_{2 x}^{\infty} R(t) d t$. The Lommel and Struve functions give useful expressions for 
integrals involving Bessel functions, thus LoMmeL 1 gave the formulae

$$
\begin{aligned}
\int_{0}^{z} t^{m} J_{n}(t) d t & =(m+n-1) z J_{n}(z) S_{m-1, n-1}(z)-z J_{n-1}(z) S_{m, n}(z) \\
\int_{0}^{z} t^{m} Y_{n}(t) d t & =(m+n-1) z Y_{n}(z) S_{m-1, n-1}(z)-z Y_{n-1}(z) S_{m, n}(z) \\
S_{m, n}(z) & =\frac{1}{2} \pi\left[Y_{n}(z) \int^{z} t^{m} J_{n}(t) d t-J_{n}(z) \int^{z} t^{m} Y_{n}(t) d t\right] .
\end{aligned}
$$

If

$$
L_{0}(x)=\sum_{n=0}^{\infty}\left(\frac{1}{2} x\right)^{2 n+1} /\left[\left(n+\frac{1}{2}\right) !\right]^{2}
$$

is the function used by NICHOLSON ${ }^{2}$

$$
\int_{0}^{x} K_{0}(t) d t=\frac{1}{2} \pi x\left[L_{0}(x) K_{1}(x)+L_{0}^{\prime}(x) K_{0}(x)\right] .
$$

This formula was used by OwEN for the computation of the integral, and preliminary tables were made of the functions $L_{0}(x), L_{0}^{\prime}(x)$.

The Lommel-Weber function may be defined by the integral

$$
-\Omega_{n}(x)=(1 / \pi) \int_{0}^{\pi} \sin (n t-x \sin t) d t
$$

and differs in sign from the function denoted by $\Omega_{n}(x)$ in AIREY 2 (corrected in AIREY 21) and by $E_{n}(x)$ in Watson 3. In terms of this function

$$
\begin{gathered}
H_{0}(x)=\Omega_{0}(x), \quad H_{1}(x)=\Omega_{1}(x)+2 / \pi \\
r_{1}(x)=x\left[\Omega_{1}(x)+2 / \pi\right] .
\end{gathered}
$$

The Poisson-Anger function in the notation of NIELSEN ${ }^{3}$ is

$$
\Psi_{n}(x)=(1 / \pi) \int_{0}^{\pi} \cos (x \sin t-n t) d t
$$

and Schläfli's function $F_{n}(x)$ is $F_{n}(x)=\int_{0}^{\infty} e^{-x \sinh u-n u} d u$; SCHLÄFLI obtained the relation ${ }^{4}$

In terms of $\Psi_{n}(x)$

$$
F_{n}(x)=(\pi / \sin n \pi)\left[\Psi_{n}(x)-J_{n}(x)\right] .
$$

$$
\begin{aligned}
\sin (n \pi) \Omega_{n}(x) & =\cos (n \pi) \Psi_{n}(x)-\Psi_{-n}(x) \\
\sin (n \pi) \Psi_{n}(x) & =\cos (n \pi) \Omega_{n}(x)-\Omega_{-n}(x) .
\end{aligned}
$$

The incomplete functions

$$
u_{p}^{r}(x)=\int_{0}^{x} \cos (r \sin t-p t) d t, \quad v_{p}^{r}(x)=\int_{0}^{x} \sin (r \sin t-p t) d t,
$$

have been discussed by $\mathrm{P}$. and E. BRAuER who have made some 6-place tables for $x / \pi=0(.05) 1, p=0(.05) .5$. 
The series $F_{1}(x), F_{3}(x), F_{5}(x), G(x)$, tabulated by GoldsteIN are

$$
G(x)=x^{2} /\left(1+x^{2}\right)-\left(8 / \pi^{2}\right) \sum_{m=0}^{\infty} F_{2 m+1}(x) /(2 m+1)^{2}
$$

where

$$
F_{2 m+1}(x)=x^{2} /\left(1+x^{2}\right)-T_{1,2 m+1}\{(2 m+1) x\} .
$$

For the series

$$
G(x)-(2 / \pi) \sum_{m=0}^{\infty} c_{m} I_{2 m+1}\{(2 m+1) x\} / I_{2 m+1}\left[(2 m+1) x_{0}\right]
$$

the quantities $c_{m}=x_{0}^{2} C_{m} /\left[\left(1+x_{0}^{2}\right) 2 m\right], C_{1}=\frac{1}{2}, C_{2}=\frac{1 \cdot 3}{2 \cdot 4}, C_{3}=\frac{1 \cdot 3 \cdot 5}{2 \cdot 4 \cdot 6}, \cdots$, $x_{0}=2(1) 10$. The series was tabulated to 3 places for $x=0(.2) 2(.5) 10$ and for 2.8(1)9.8. For the value $x_{0}=5, p=2$, a table is formed for the series

$$
\begin{gathered}
-\left[x^{2} /\left(1+x^{2}\right)\right]-\left(8 / \pi^{2}\right) \sum_{m=0}^{\infty} F_{p, 2 m+1}(x) /(2 m+1)^{2} \\
-(2 / \pi) \frac{x_{0}^{2}}{1+x_{0}} \sum_{m=0}^{\infty} A_{m} I_{p(m+\xi)}\left\{\left(m+\frac{1}{2}\right) p x\right\} / I_{p(m+\xi)}\left\{\left(m+\frac{1}{2}\right) p x_{0}\right\}
\end{gathered}
$$

where $A_{0}=1,3 A_{1}=\frac{1}{2}, 5 A_{2}=\frac{1 \cdot 3}{2 \cdot 4}, 7 A_{3}=\frac{1 \cdot 3 \cdot 5}{2 \cdot 4 \cdot 6}, \cdots$ The range is $x=.1, .2(.2) 2(.5) 5$; a value is also given for $x=4.8$.

1 E. LOMmel, Math. Annalen, v. 9, 1876, p. 425-444.

$2 \mathrm{~J}$. W. Nicholson, Quar. J. Math., v. 42, 1911, p. 216-224.

3 N. NiELSEN, Handbuch der Theorie der Cylinderfunktionen, Leipzig, 1904.

4 L. SCHLÄFLI, Math. Annalen, v. 10, 1876, p. 137-142.

$$
\mathbf{A}_{1} . \mathbf{\Omega}_{0}(x)=H_{0}(x), \mathbf{\Omega}_{1}(x), H_{1}(x)
$$

1. $7 \mathrm{D}, \Omega_{0}(x)$, Watson $3, x=0(.02) 16$

2. 7D, $H_{1}(x)=\Omega_{1}(x)+2 / \pi$, WATSON $3, x=0(.02) 16$

3. $6 \mathrm{D}, \Omega_{0}(x), \Omega_{1}(x)=H_{1}(x)-(2 / \pi)$, AIREY 21, p. 280f, $x=0(.02) 16$

4. $4 \mathrm{D}, \Omega_{0}(x), \Omega_{1}(x), H_{1}(x)$, JAHNKE \& EMDE $1_{8}-1_{5}, x=0(.01) 14.99, \Delta$

5. $4 \mathrm{D}, H_{0}(x), H_{1}(x)$, MCLAChlaN 2 , p. $176, x=0(.1) 15.9$

6. $4 \mathrm{D}, H_{0}(x), H_{1}(x)$, GLaZENAP, $x=0(.02) 16$

7. $4 \mathrm{~S}, H_{0}(x), 3$ zeros, McLachlan 2 , p. 66

8. $4 \mathrm{D}, 2 H_{1}(x) / x[=M(x)]$, MORSE, p. $337, x=0(.1) \cdot 2(.2) 4(.5) 10(1) 15$

9. $3 \mathrm{D}, \alpha_{0}, \beta_{0}$, in $\tanh \pi\left(\alpha_{0}+i \beta_{0}\right)=1-(2 / x) J_{1}(x)-2 i H_{1}(x) / x$, MORSE, p. 337, $x=0(.1) .2(.2) 4(.5) 10(1) 15$

10. $2 \mathrm{D}, 2 x H_{1}(2 x)=r_{1}(2 x)$, MCLACHLAN \& SOWTER, $x=.25(.25) 1(.5) 10$

11. $4 \mathrm{D} ; 5 \mathrm{D}, \pi H_{1}(x) / 2 x^{2}=R(x)$, MiLne \& FowleR, $x=0(.5) 5(1) 8 ; 9,10,12$. Also a graph $0<x<7$

12. $2-3 \mathrm{D},[n R(x)]^{2}$, MILNE \& FoWLER, $n=1(1) 5,2 x=1(1) 4(2) 12$

13. $3 \mathrm{D},\left(3 x^{8} / \pi\right) R^{2}(x)$, MILNE \& FOWLER, $2 x=1(1) 4,6$

$$
\mathbf{A}_{2} . \boldsymbol{\Omega}_{n}(x) \text { and its functions }
$$

1. $6 \mathrm{D}, \Omega_{3}(x), \Omega_{-3}(x)$, Airey $25, x=0(.02) 20$

2. $6 \mathrm{D}, \Omega_{n}(n), \Omega_{n-1}(n)$, AIREY $20,4 n=0(1) 40$

3. $6 \mathrm{D}, \Omega_{2 m-1}(2 m)-\Omega_{2 m+1}(2 m)$, AIREY $16, m=1(1) 15$

4. 6D, $F_{ \pm n}(n), F_{ \pm(n-1)}(n)$, AIREY $20,4 n=0(1) 40$ 


\section{B. Miscellaneous}

1. $4 \mathrm{D},\left[x^{2} /\left(1+x^{2}\right)\right]-T_{1, n}(n x)$, and $G(x)$, Goldstein, p. $446, n=1,3,5$, $x=.2(.2) 2(.5) 5(1) 9$

2. 6-7S, $L_{0}(x), L_{0}^{\prime}(x)$, OWEN, $x=.02, .1, .5,1(1) 12$

\section{Graphs}

1. $\Omega_{n}(x)$, JAHNKE \& EMDE $18-1_{5}$ (fig. 116), $0<x<16$

2. $\sin \psi=\left\{1 /\left[J_{0^{2}}(x)+\Omega_{0}^{2}(x)\right]\right\}\left[.5 x J_{0}(x) \pm \Omega_{0}(x)\left(J_{0}^{2}(x)+\Omega_{0}^{2}(x)+.25 x^{2}\right)^{3}\right]$, GRAMMEL 3 , $0<\psi<360^{\circ}$

3. $H_{0}(x), H_{1}(x)$, McLachlan $2,0<x<16$

4. $H_{0}(x), H_{1}(x)$, JAHNKe \& EMDE $1_{3}-1_{b}$ (fig. 117), $0<x<15$

5. $J_{0}(x), H_{0}(x),\left[J_{0}{ }^{2}(x)+H_{0}{ }^{2}(x)\right]^{3}$, MENGES

6. $x H_{1}(x)=r_{1}(x)$, MCLACHLAN $1,0<x<10$

7. $1-J_{1}(2 x) / x$ and $r_{1}(2 x) / 2 x^{2}=H_{1}(2 x) / x$, A. H. DAvis, $0<x<15$

See also IX $A_{1} 11$

\section{Integrals Involving Bessel Functions}

In this Section it will be convenient to use a bar notation and write

$$
\begin{aligned}
& \bar{J}_{n}(x)=\int_{0}^{x} J_{n}(t) d t, \quad \bar{Y}_{n}(x)=\int_{0}^{x} Y_{n}(t) d t, \quad \bar{I}_{n}(x)=\int_{0}^{x} I_{n}(t) d t, \\
& \bar{K}_{n}(x)=\int_{0}^{x} K_{n}(t) d t, \bar{I}_{m, n}(x)=\int_{0}^{x} u^{m} I_{n}(u) d u, \bar{K}_{m, n}(x)=\int_{0}^{x} u^{m} K_{n}(u) d u .
\end{aligned}
$$

Use will also be made of the notation of BICKLEY and NAYLER

$$
\begin{aligned}
K i_{n}(x) & =\int_{0}^{\infty} \exp (-x \cosh u) \operatorname{sech}^{n} u d u=\int_{x}^{\infty} K i_{n-1}(t) d t \\
& =\int_{x}^{\infty}(t-x)^{n-1} K_{0}(t) d t /(n-1) !
\end{aligned}
$$

The integral of SEELIGER

$$
\int_{0}^{1} \exp \left[-x\left(1-t^{2}\right)^{-1}\right] d t
$$

is seen to be $K i_{2}(x)$. Use will be made also of the notation

$$
\begin{aligned}
& g(x)=\int_{0}^{x} J_{1}(t) d t / t, \quad j(x)=\int_{0}^{x} J_{1}{ }^{2}(t) d t / t \\
& h(x)=(1 / \pi) \int_{x}^{\infty} H_{1}(t) d t / t^{2}=\int_{2 x}^{\infty} R(t) d t \quad \text { (Struve's integral). }
\end{aligned}
$$

The integrals of Havelock 1,

$$
\begin{aligned}
P_{2 n}(x) & =(-1)^{n} \int_{0}^{\pi / 2} \cos ^{2 n} t \sin (x \sec t) d t \\
P_{2 n+1}(x) & =(-1)^{n+1} \int_{0}^{\pi / 2} \cos ^{2 n+1} t \cos (x \sec t) d t
\end{aligned}
$$


are expressible in terms of $Y_{0}(x), Y_{1}(x)$, and $\bar{Y}_{0}(x)$, while $\bar{Y}_{0}(x)$ is calculated from the formula

$$
\bar{Y}_{0}=x Y_{0}-\frac{1}{2} \pi x\left(Y_{0} H_{1}-Y_{1} H_{0}\right) \text {. }
$$

In particular

$$
P_{0}(x)=-\frac{1}{2} \pi \bar{Y}_{0}(x), \quad P_{0}^{-1}(x)=\int_{0}^{x} P_{0}(t) d t=1+P_{1}(x) .
$$

Havelock also writes

$$
Q_{1}(x)=\int_{0}^{x} Q_{0}(t) d t, \quad \text { where } \quad Q_{0}(x)=\frac{1}{2} \pi \int_{0}^{x}\left[H_{0}(t)-Y_{0}(t)\right] d t .
$$

His integrals $I_{3}$ and $I_{5}$ may be written in the form

$$
I_{2 n+1}=F_{n}(0)-2 F_{n}(x)+F_{n}(2 x) ;
$$

$$
\begin{aligned}
F_{n}(x)=\int_{0}^{\infty} \exp .\left(-x \cosh ^{2} u\right) d u / \cosh ^{2 n} u & \\
& =1 /[2(n-1) !] \int_{x}^{\infty}(t-x)^{n-1} e^{-\frac{1}{2} t} K_{0}\left(\frac{1}{2} t\right) d t .
\end{aligned}
$$

Some integrals which originated in problems treated by EULER ${ }^{1}$ in 1743 and 1781, and now called Fresnel Integrals, are defined as follows:

$$
\begin{aligned}
& S(u)=\int_{0}^{u} \sin \left(\frac{1}{2} \pi t^{2}\right) d t=\frac{1}{2} \int_{0}^{x} J_{3}(t) d t \\
& C(u)=\int_{0}^{u} \cos \left(\frac{1}{2} \pi t^{2}\right) d t=\frac{1}{2} \int_{0}^{x} J_{-3}(t) d t
\end{aligned}
$$

where $x=\frac{1}{2} \pi u^{2}$. There are a number of tables for $S(2 x / \pi)^{\frac{1}{3}}$, and $C(2 x / \pi)^{\frac{1}{3}}$. See also Section XII.

The Integrals used by Schwarz may be defined as follows:

$$
\begin{array}{ll}
J c(k, x)=\int_{0}^{x} J_{0}(k u) \cos u d u, & J s(k, x)=\int_{0}^{x} J_{0}(k u) \sin u d u \\
Y c(k, x)=\int_{0}^{x} Y_{0}(k u) \cos u d u, & Y s(k, x)=\int_{0}^{x} Y_{0}(k u) \sin u d u .
\end{array}
$$

They are connected by the relations

$$
\begin{aligned}
& C c(k, x)=Y c(k, x)-(2 / \pi) \ln (k x) J c(k, x) \\
& C s(k, x)=Y s(k, x)-(2 / \pi) \ln (k x) J s(k, x)
\end{aligned}
$$

with two other functions which have been tabulated.

An integral used by KüSSNER is defined by the equation

where

$$
S(x)=\left(\frac{1}{2} i \pi\right) x \int_{x}^{\infty} G_{1}(u) d u / u
$$

$$
G_{1}(u)=H_{1}(i u)-Y_{1}(i u)-2 j \pi \text {. }
$$


The integral used by KING will be defined as follows:

$$
K e(x)=\int_{0}^{x} e^{u} K_{0}(u) d u .
$$

1 See R. C. ARchibald, "Euler integrals and Euler's spiral-sometimes called Fresnel integrals and the clothoïde or Cornu's spiral," Am. Math. Mo., v. 25, 1918, p. 276-282. (On p. 280, 1. 2 and 14, for Peters, read Gilbert.) Some tables not listed in the present text are there indicated.

\section{A. Integrals involving $J_{n}(x), Y_{n}(x)$}

1. $10 \mathrm{D}, \bar{J}_{0}(x), \bar{Y}_{0}(x)$, LOWAN \& ABRAMOWITZ, $x=0(.01) 10$

2. 7D, $\frac{1}{2} \bar{J}_{0}(x), \frac{1}{2} \bar{Y}_{0}(x)$, WATSON $3, x=.02(.02) 1$

3. $7 \mathrm{D}, \frac{1}{2} \bar{J}_{0}(x) ; \frac{1}{2} \bar{Y}_{0}(x)$, WATSON $3, x=x_{s} ; y_{s}, s=1(1) 16$

4. $4 \mathrm{D}, \frac{1}{2} \bar{J}_{0}(x), \frac{1}{2} \bar{Y}_{0}(x)$, GLAZENAP, $x=.02(.02) 1$

5. $4 \mathrm{D},\left|\int_{0}^{v} e^{j i \pi u^{2}} J_{0}\left(\alpha u^{3}\right) d u\right|^{2}$, SiLBERSTEIN, $\alpha=0$, and $\alpha\left[10^{3 / 2} /(2 \pi)\right]=1,10(10) 30 ; v=0(.1) 3$

6. $10 \mathrm{D} ; 12 \mathrm{D}, J i_{0}(x)=\int_{x}^{\infty} J_{0}(t) d t / t, F(x)=J i_{0}(x)+\ln (x / 2)$, Lowan, Blanch \& ABramowitz, $x=0(.1) 3$, with $\delta^{2}, \delta^{4}, \delta^{6}$ for $F(x)$; also, T. II : $J i_{0}(x)$, with $\delta^{2}, \delta^{4}$ (modified), $x=3(.1) 7$; also T. III : reduced derivatives of $F(x)$, values of $A_{n}(x)=F^{(n)}(x) / n !$, $A_{0}(x)=F(x), n=0(1) 13, x=10(1) 21$; T. II to $10 \mathrm{D}$; T. III to $12 \mathrm{D}$

7. $4 \mathrm{D}, \int_{i 1,1}^{\infty} J_{1}(t) d t / t$, WILTON

8. $\leq 4 \mathrm{D}, g(x)$, Gans $4, x=0(.1) 14$, 15. Also $W^{2}=[g(x+y)-g(x-y)]^{2}$ (a) $y=1$, $x=0(.2) 10$; (b) $y=2.6, x=[0(.2) 11.4 ; 3-4 \mathrm{D}]$; (c) $y=4, x=[0(.5) 10 ; 3-4 \mathrm{D}]$; (d) $y=7, x=[0(.4) 6.8,7,8 ; 3 \mathrm{D}$ or $4 \mathrm{~S}]$; graphs for (a)-(d)

9. 3D or $4 \mathrm{~S}, W=W_{0}-2 \int_{0}^{\infty} \cos (x t) J_{1}(t) d t / t, W_{0}=2\left(1-x^{2}\right)^{3}, x<1 ; W_{0}=0, x \geq 1$, Gans 4 , p. 19 , table of $W_{1}, W_{1}^{2}, W_{0}^{2}, x=0(.2) 1(.1) 1.8,2$

10. $6 \mathrm{D}$, Integrals from 0 to 1 for each of the following functions: (a) $1 / J_{0}(x)$; (b) $x / J_{1}(x)$; (c) $\ln J_{0}(x)$; (d) $x J_{0}(x) / J_{1}(x)$; (e) $J_{1}(x) / J_{0}(x)$; (f) $J_{2}(x) / J_{1}(x)$; (g) $\ln \left|J_{0}(x)\right|$; (h) $\ln \left|2 J_{1}(x) / x\right|$; (i) $\ln \left|1 / J_{0}(x)\right|$; (j) $\ln \left|x / 2 J_{1}(x)\right|$; (k) $\ln \left|8 J_{2}(x) / x^{2}\right|$; (l) $\ln \left|x^{2} / 8 J_{2}(x)\right|$, BRIXY 2

11. $4 \mathrm{D}$, Twice the integrals from .5 to 1 of (a) $J_{0}\left(4 v^{3 / 2}-3 v^{3}\right)$, (b) $J_{0}\left(6 v^{3 / 2}-4.75 v^{\mathrm{j}}\right)$, (c) $J_{0}(1 \cdot 2 v)$, STEWARD, p. 175, 177; also graphs of $\left[1 /\left(1-a^{2}\right)\right] \int_{a^{2}}^{1} J_{0}\left(4 v^{3 / 2}-z v^{3}\right) d v, a^{2}=0, \frac{1}{2}, 1$

12. $2 \mathrm{D},\left[\int_{0}^{x} J_{0}(v) d v-J_{1}(x)\right] / J_{1}(x)$, SCHELKUNOFF, p. $324, x=1.841$

13. 7D, $n \int_{0}^{1} J_{n}(n x) d x$, WATson $2, n=1(2) 23$

14. $4 \mathrm{D}, F_{0}(k r)=\int_{0}^{\infty} J_{0}(m r) d m /(m+k)$, and $1-k r F_{0}(k r)$, to 3D, LAMB 7, $k r=0(.05) \cdot 1(.1) 1$

15. $4 \mathrm{D}, F_{1}(k r)=\int_{0}^{\infty} m J_{1}(m r) d m /[k(m+k)]$, and $1-(k r)^{2} F_{1}(k r)$, to 3D, LAMB 7, $k r=0(.05) \cdot 1(.1) 1$

16. $4 \mathrm{D}, \int_{0}^{\infty} J(m r) m(m-n) d m /[(m+a)(m+b)]$, LAMB $7, n(a-b)=a b, a=5 b$, $a r=0(.05) .1(.1) 1$

17. $4 \mathrm{D}, h(x)$, Struve $2, x=0(.1) 4(.2) 7(.4) 15$

18. 6D, $j(x)-j(y)$, Steiner, where the 49 values of $x$ and $y$ are maxima ( 0 to 154.72331) of $J_{1}(t) / t$

19. 3D, $I=\int_{0}^{\infty} e^{-x u^{2}} d u / u\left[J_{0}^{2}(u)+Y_{0}^{2}(u)\right]$, JAEGER \& CLARKE, $x=0(.01) 1(.1) 10(1) 100(10) 1000$

20. $4 \mathrm{~S},(8 / \pi) I$ (no. 19), Perry \& Berggren, p. 70, $x=.001, .005, .01, .02, .05, .1$, $.2(.2) 1(.5) 3.5$. There is also, on p. 72, a table of values (to $4 \mathrm{D}$ ) of a much more complicated integral involving $J_{0}$ and $Y_{0}$. 
21. 6D, $J c(k, x), J s(k, x),-Y c(k, x),-Y s(k, x)$, SchwARZ, $k=.1(.1) 1, x=0(.02) 2$

22. $8 \mathrm{D}, J c(k, x), J_{s}(k, x)$, SchWARZ, $k=.1(.1) 1, x=0(.1) 5$

23. $6 \mathrm{D}, Y c(k, x), Y s(k, x)$, Schwarz, $k=.1(.1) 1, x=2(.1) 5$

24. $6 \mathrm{D},-C_{c}(k, x),-C_{s}(k, x)$, SchwARz, $k=0, .1, x=0(.02) 1.58 ; k=.2, x=0(.02) .88$; $k=.3, x=0(.02) .62 ; k=.4, x=0(.02) .48 ; k=.5, .6, .7, x=0(.02) .38 ; k=.8, .9$, $x=0(.02) .28 ; k=1, x=0(.02) .2$

25. $3 \mathrm{D},(2 / \pi) \int_{0}^{\infty}\left[K_{0}(t)\right]^{2} d t / K_{0}(x t)$, OLLENDORFF $1, x=.001, .01, .05, .1(.1) 5$; also for this range of $x$ the same integral with the limits .1 to 3

26. $3 \mathrm{D},(2 / \pi) \int_{0}^{\infty} K_{1}(t) K_{0}(t) t d t / K_{0}(x t)$, OLLENDORFF $1, x=.001, .01, .05, .1(.1) .5$

27. $5 \mathrm{D},\left(\frac{1}{2} i \pi\right) x \int_{x}^{\infty}\left[H_{1}(i u)-Y_{1}(i u)-2 / \pi\right] d u / u$, KüSSNER, real and imaginary parts $x=0(.02) .6(.1) 1(.2) 6(1) 10(2) 14,20, \infty$

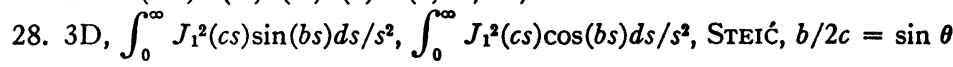
$\theta=0\left(5^{\circ}\right) 30^{\circ}\left(10^{\circ}\right) 90^{\circ}$

29. $4 \mathrm{D}, P_{n}(x), n=3,4,5$, Havelock $1, x=0(.4) 4.8,5(1) 10$

30. 7D, $g(m, n)=\int_{0}^{\infty} e^{-x t} J_{m}(t) J_{n}(t) d t / t$, NomURA $2,(m, n)=(1,0),(1,1),(2,0),(2,1)$, $(2,2),(3,0),(3,1),(3,2),(3,3),(4,0),(4,1),(4,2),(4,3),(4,4),(5,0),(5,1),(5,2)$, $(5,3),(6,0),(6,1)$, for $x=.4(.2) 1.2,1.5(.5) 3,5$ and 10,20 in some cases. There are also tables, to $5 \mathrm{D}$, of $G_{p}{ }^{k}=(4 k+2) g(2 p+1,2 k+1)$ and of $G_{1, p}^{k}=(4 k+4) g(2 p+2,2 k+2)$. In NomURA 1 and 3 the tables of $g(m, n)$ are also to $7 \mathrm{D}$, and $m$ and $n$ are halves of integers, $m+n$ varying from 1 to 8 . In Nomura 1 there are also tables, to $5 \mathrm{D}$, of $G_{p}{ }^{k}$, $G_{1, p}^{k}$, where now, for example, $G_{1, p}^{k}=(4 k+5) g(2 p+3 / 2,2 k+3 / 2)$.

31. $5 \mathrm{~S}, \bar{S}_{2 n}(x),(-1)^{n} \bar{C}_{2 n}(x)$, STENZEL, $x=1(.25) 10$

\section{A2. Fresnel integrals $S(u)$ and $C(u)$}

1. $4 \mathrm{D}$, FRESNEL, $u=0(.1) 5.5$

2. $4 \mathrm{D}$, AIRY $1, u=0(.1) 5.5$

3. $4 \mathrm{D}$, IgNatowsKy, and JAHNKe \& EMDE $1_{1}$ (p. 26) $-1_{6}$ (p. 34), $u=0(.1) 8.5$

4. $6 \mathrm{D}, \log S(u), \log C(u)$, IGNATOWSKY, $u=0(.1) 8.5$

5. $4 \mathrm{D}, \frac{1}{2}\left[S^{2}(u)+C^{2}(u)\right]$, IGNATOWSKY, $u=0(.1) 8.5$

6. $4 \mathrm{D}, C^{2}(u)+S^{2}(u)$, Silberstein, $u=0(.1) 3$

7. $3 \mathrm{D}, 2^{-i} S(u), 2^{-i} C(u)$, Bouman \& JoNG, $u=0(.1) 8.5$ [argument $2.5 u=100 x$ ]

8. $4 \mathrm{D}, \frac{1}{4}\left\{[1+C(u)+S(u)]^{2}+[C(u)-S(u)]^{2}\right\}$, MAYALL, $u=.01(.01) .1(.05) 1(.1) 5.5$; also $\frac{1}{2}\left\{[1-C(u)-S(u)]^{2}+[C(u)-S(u)]^{2}\right\}, u=.05(.05) 1$

\section{A 8. Fresnel integrals $S\left[(2 x / \pi)^{3}\right], C\left[(2 x / \pi)^{\natural}\right]$}

1. 7D, Watson $3, x=0(.02) 1$

2. $6 \mathrm{D}$, Lommel $3, x=0(.1) 1(.5) 50$; also interpolation tables, and maxima to $6 \mathrm{D}, x=n \pi$ for $S$, and $x=\left(n-\frac{1}{2}\right) \pi$ for $C, n=1(1) 16$

3. 6D, Watson $3, x=0(.5) 50$; also maxima to $6 \mathrm{D}, x=n \pi$ for $S$, and $x=\left(n-\frac{1}{2}\right) \pi$ for $C, n=1(1) 16$

4. $6 \mathrm{D}$, Airey $26, x=0(.1) 20$

5. 5D, Hayashi $2, x=0(.02) 1(.5) 50$; also maxima to $5 \mathrm{D}, x=n \pi$ for $S$, and $x=\left(n-\frac{1}{2}\right) \pi$ for $C, n=1(1) 16$

6. $4 \mathrm{D}$, JAHNKe \& EMdE $1_{1}$ (p. 25) $-1_{5}$ (p. 35), $x=0(.1) 1(.5) 50$

\section{B. Integrals involving $I_{n}(x), K_{n}(x)$}

1. $8 \mathrm{D}, \bar{I}_{m}(x), m=1,2$, HAY, $x=2.8$

2. 7D, $\bar{I}_{0}(x)$, BURSIAN \& FOK, $x=0(.1) 6$

3. $7 \mathrm{D}, e^{-x} \bar{I}_{0}(x)$, BURSIAN \& FoK, $x=0(.1) 16$

4. $6 \mathrm{~S}, \bar{I}_{0}(x)$, R. MÜLLER, $x=0(.1) 5.2(.2) 16, \delta^{2}$ 
5. $8 \mathrm{D}, \int_{0}^{2.8} x^{t} I_{2 n}(x) d x, \mathrm{HAY}, n=2, t=1,2$

6. 7D, $(1 / \pi) \int_{0}^{\infty} t^{p} d t / I_{n}^{2}(t)$, Warson $4, n=0,1 ; p=2,4$

7. 3D, $F(x)=8 \pi^{-i} e^{-x^{2}} \int_{0}^{\infty} t^{2} e^{-t^{2}} I_{0}(2 x t) d t$ and $\left[\pi^{3} / 2 x\right] F(x)$, WAGNER, $x=0(.1) 1(.2) 3$

8. 7D; 8D; 9D; 10D; 11D; 12D, $\bar{K}_{0}(x)=\frac{1}{2} \pi-K i_{1}(x)$, BuRSIAN \& FoK, $x=0(.1) 1.3$; $1.4(.1) 3.3 ; 3.4(.1) 5.4 ; 5.5(.1) 7.6 ; 7.7(.1) 9.8 ; 9.9(.1) 12$

9. $7 \mathrm{D}, e^{x} \hat{K}_{0}(x)$, BURSIAN \& FoK, $x=0(.1) 16$

10. 7D-6D, $\vec{K}_{0}(x)=\frac{1}{2} \pi-K i_{1}(x)$, OWEN, $x=.02, .1, .5,1(1) 12, \infty(\pi / 2)$

11. $5 \mathrm{D}, \int_{0}^{x} u K_{1}(u) d u=\vec{K}_{0}(x)-x K_{0}(x)$, ChANDRASEKHAR, $x=0(.1) 4.5, \infty$

12. $4 \mathrm{~S}, \hat{K}_{0}(x)$, Віот, $x=0(.1) \cdot 2, .5,1(1) 5$

13. $6 \mathrm{D} ; 4 \mathrm{D} ; 3 \mathrm{D}, K e(x)=\int_{0}^{x} e^{t} K_{0}(t) d t$, KING, $x^{3}=.01(.01) .1,5(1) 10$; $x=.02(.01) .1(.1) 3(.2) 6(.5) 10(1) 20 ; x=5(1) 10 ;$ also $x / K e(x)$, to $4 \mathrm{D}$, throughout the range

14. 6D, $T_{m}(x)$, DAvid \& Fieller, $m=0(.5) 11.5$, $x=0(.1) 4(.5) 18\left[T_{m}(x)=\pi^{-\frac{1}{3} x^{m}} K_{m}(x) /\left\{2^{m}\left(m-\frac{1}{2}\right) !\right\}\right]$

15. $9 \mathrm{D}, K i_{n}(x)$, BICKLEY \& NAYLER, $n=1(1) 16, x=0(.05) .2(.1) 2,3$

16. $6 \mathrm{D}, K i_{2}(x)$, SEELIGER, $x=.25(.25) 7$

17. $3 \mathrm{D}, 1-\phi(t)=\int_{0}^{\infty} e^{-x t} d t /\left[t^{2}\left(D^{2}+\pi^{2} E^{2}\right)\right], \psi(t)=1-\int_{0}^{\infty} e^{t-x t} E d t /\left[t^{2}\left(D^{2}+\pi^{2} E^{2}\right)\right]$, SEARS, $D=K_{0}(t)-K_{1}(t), E=I_{0}(t)+I_{1}(t), x=0\left(\frac{1}{t}\right) 1\left(\frac{1}{2}\right) 2(2) 10(5) 30$, 40; graphs of $1-\phi(t)$ and $\psi(t)$

18. $4 \mathrm{D}, I_{p}=(1 / \pi) \int_{0}^{2 a}\left[1-J_{0}^{2}(r)-J_{1}^{2}(r)\right] d r /\left(4 a^{2}-r^{2}\right)^{1}$ $=\frac{1}{2}-(1 / \pi) \int_{0}^{2 a}\left[J_{0}^{2}(r)+J_{1}^{2}(r)\right] d r /\left(4 a^{2}-r^{2}\right)$, tables of $I_{p}, \frac{1}{2}-I_{p}$, NAGAOKA 3 , T. I, $a=20(5) 50(10) 100(50) 300(100) 1000(500) 3000$

19. $4 \mathrm{D}, S=\left(a k^{\prime} / \pi\right) \int_{a k^{\prime}}^{x_{1}}\left\{1-J_{0}^{2}(x)-J_{1}^{2}(x)\right\} d x /\left[k x\left(x^{2}-a^{2} k^{\prime 2}\right)^{3}\right]$, NAGAOKA 3, T. IV, $2 a k^{\prime}=0(.1) 3.8,3.8317 ; k, k^{\prime}, x_{1}$, connected with an elliptic integral in the derivation

20. $4 \mathrm{D}, I_{2}=\int_{0}^{3 \pi}\left\{1-J_{0}^{2}\left[a(1+\nu)\left(1-k^{2} \sin ^{2} \psi\right)^{3}\right]-J_{1}^{2}\left[a(1+\nu)\left(1-k^{2} \sin ^{-2} \psi\right)^{\natural}\right]\right\} k^{\prime} d \psi /$ $\left(1-k^{2} \sin ^{-2} \psi\right)$, NAGAOKA 3, T. VII, $2 a k^{\prime}=.1(.1) 3.8,3.8317 ; k^{\prime}=|1-\nu| /|1+\nu|$ $=\left(1-k^{2}\right)^{3}$, are connected with an elliptic integral in the derivation

\section{Graphs}

1. $(1 / x) J_{0}(x)$, F. A. Fischer, $0<x<15$

2. $(1 / x) \bar{J}_{0}(x)$, DEBYE, $0<x<20$

3. $J_{0}(x)$, BLACKMAN, $0<x<2.8$

4. $J_{c}(k, x), J_{s}(k, x), Y c(k, x), Y s(k, x), 0<k \leq 1,0 \leq x \leq 5$, SchwARz; see XA $20-22$

5. $\int_{0}^{\pi} J_{1}(x u \cos \psi) \sin u d u$, LABUS, $x=.4(.1) \cdot 6(.2) 1, \psi=0\left(10^{\circ}\right) 90^{\circ}$

6. $\int_{0}^{1} J_{0}\left(4 u^{3 / 2}-z u^{\dagger}\right) d u$, STEWARD, p. $154,184,-10<z<+10$

7. $e^{-x}\left[1+\int_{0}^{\sqrt{8 x z}} \exp \left\{-t^{2} /(8 x)\right\} I_{1}(t) d t\right]$, NORDSIECK, LAMB \& UHLENBECK $z=7$, $x=0(2) 12 ;$ and $z=4.75, x=0(4) 20$

8. $\left\{\left[x^{2}\left(3-a^{2}\right) / 12\right] /\left[a x I_{0}(x) / \bar{I}_{0}(a x)-1\right]\right\}-1$, RUDOLPH, $0<x<11, a=0, .8, .9, .95,1$

9. JAEGER, $I\left(\frac{1}{2} \pi k^{-1}, \frac{1}{2} \pi k^{-1} ; a\right)$, fig. 1, JAEGER, $a=0(2) 10 ; k=[.2, .5,1,2.5,5,10]$, where $I(p, q ; x)=\int_{0}^{\infty} \exp \left(-x u^{2}\right) d u /\left[u\left(J^{2}+Y^{2}\right)\right], J=p u J_{1}(u)+q J_{0}(u)$,

$Y=p u Y_{1}(u)+q Y_{0}(u)$; also, fig. $2, \lim _{q \rightarrow 0}[I(1, q ; 0)-I(1, q ; a)]$

10. Contours of $\int_{0}^{1} J_{0}\left\{\left(x^{2}+y^{2}\right) v-8 y v^{2}+16 v^{3}\right\} d v=$ const, STEWARD, p. $156,|x|<10$, $|y|<10$

11. $F_{n}(0)-2 F_{n}(x)+F_{n}(2 x)$, Havelock $2, n=2,3,0<?<x<$ 
12. $(1 / 4 k)\left[Q_{1}(p+k)-Q_{1}(p)\right]$, Havelock $3, k=0(1) 3, p=-4(1) 3$

13. $(1 / k)\left[P_{0}^{-1}(p-k)-P_{0}^{-1}(p)\right]$, HaVelock $3, k=0(.5) 1(1) 6, p=0(1) 10$

14. $\theta_{0}(x)=(2 / \pi) \int_{0}^{\infty}\left[K_{0}(t)\right]^{2} d t / K_{0}(x t)$, OLLENDORFF 1 , also $1 /\left[2 \theta_{0}(x)\right], x=0(.1) 1$, and $x=0(.02) .2$

15. $\theta_{0}(x, y)=(2 / \pi) \int_{0}^{\infty} K_{0}(t) \cdot K_{0}(y t) d t / K_{0}(x t)$, OLLENDORFF $1, x=.01, .05, .1(.1) .5$ $y=0(.1) 1$

See also $X \mathrm{~A}_{1} 8,11 ; \mathrm{X} \mathrm{B} 17$

\section{Bessel Functions of a Complex Argument}

The function $I_{0}\left(x i^{\frac{1}{3}}\right)$ occurred in 1850 in the work of STokes 1 on the effect of the internal friction of fluids on the motion of pendulums, and again in 1889 in the work of KELVIN ${ }^{1}$ on electric currents. Bessel functions of a complex argument soon became well-known to electricians and were found to be useful also in hydrodynamics and the conduction of heat. Kelvin's ber and bei notation has been adopted with some modifications and extensions which may be described as follows:

$$
\begin{aligned}
& \operatorname{ber}_{n} x+i \operatorname{bei}_{n} x=M_{n}(x) e^{i \theta_{n}(x)}=J_{n}\left(x i^{3 / 2}\right)=i^{n} I_{n}\left(x i^{\frac{1}{3}}\right) \\
& \operatorname{ker}_{n} x+i \operatorname{kei}_{n} x=N_{n}(x) e^{i \phi_{n}(x)}=i^{n} K_{n}\left(x i^{\frac{1}{2}}\right)=\frac{1}{2} \pi i H_{n}(1)\left(x i^{3 / 2}\right) \\
& \operatorname{her}_{n} x+i \operatorname{hei}_{n} x=H_{n}(x)\left(x i^{3 / 2}\right), \quad \operatorname{yer}_{n} x+i \operatorname{yei}_{n} x=Y_{n}\left(x i^{3 / 2}\right)
\end{aligned}
$$

In particular, for example,

$$
\text { ber } x+i \text { bei } x=I_{0}\left(x i^{1}\right), \quad \text { yer } x+i \text { yei } x=Y_{0}\left(x i^{3 / 2}\right)
$$

The functions $\operatorname{ker} x$ and kei $x$ were introduced by Russell in 1909 and the more general functions $\operatorname{ber}_{n} x, \operatorname{bei}_{n} x, \operatorname{ker}_{n} x, \operatorname{kei}_{n} x$ by WHITE$\operatorname{HEAD}^{2}$ in 1911. The terms $\operatorname{her}_{n} x, \operatorname{hei}_{n} x, \operatorname{yer}_{n} x, \operatorname{yei}_{n} x$ were added by $\mathrm{J}$. C. P. Miller in the Liverpool Index. We shall not use the notation $\operatorname{ster}_{n} x+i$ stei $_{n} x=H_{n}\left(x i^{3 / 2}\right)$, offered by McLachlan \& MEYers.

In such a table as NYMTP 3 , for $J_{0}(z)$ and $J_{1}(z)$ if $z=r e^{i \theta}$

$$
J_{0}(z)=u_{0}(r, \theta)+i v_{0}(r, \theta), \quad J_{1}(z)=u_{1}(r, \theta)+i v_{1}(r, \theta),
$$

then

$$
\begin{aligned}
\text { ber } x & =u_{0}\left(x, \frac{1}{4} \pi\right), & \text { bei } x & =-v_{0}\left(x, \frac{1}{4} \pi\right), \\
\operatorname{ber}_{1} x & =-u_{1}\left(x, \frac{1}{4} \pi\right), & \text { bei }_{1} x & =v_{1}\left(x, \frac{1}{4} \pi\right) .
\end{aligned}
$$

Similarly, in NYMTP 6, if

$$
Y_{0}(z)=U_{0}(r, \theta)+i V_{0}(r, \theta), \quad Y_{1}(z)=U_{1}(r, \theta)+i V_{1}(r, \theta),
$$

then

$\operatorname{ker} x=-\frac{1}{2} \pi\left[U_{0}\left(x, \frac{1}{4} \pi\right)+v_{0}\left(x, \frac{1}{4} \pi\right)\right], \quad$ kei $x=\frac{1}{2} \pi\left[V_{0}\left(x, \frac{1}{4} \pi\right)-u_{0}\left(x, \frac{1}{4} \pi\right)\right]$, $\operatorname{ker}_{1} x=\frac{1}{2} \pi\left[U_{1}\left(x, \frac{1}{4} \pi\right)+v_{1}\left(x, \frac{1}{4} \pi\right)\right], \quad \operatorname{kei}_{1} x=\frac{1}{2} \pi\left[-V_{1}\left(x, \frac{1}{4} \pi\right)+u_{1}\left(x, \frac{1}{4} \pi\right)\right]$.

It will also be useful to note that

$$
\begin{array}{ll}
\operatorname{ber}^{\prime} x=\left[\operatorname{bei}_{1} x+\operatorname{ber}_{1} x\right] / 2^{\frac{1}{2}}, & \operatorname{bei}^{\prime} x=\left[\operatorname{bei}_{1} x-\operatorname{ber}_{1} x\right] / 2^{\frac{1}{3}}, \\
\operatorname{ker}^{\prime} x=\left[\operatorname{kei}_{1} x+\operatorname{ker}_{1} x\right] / 2^{\frac{1}{2}}, & \operatorname{kei}^{\prime} x=\left[\operatorname{kei}_{1} x-\operatorname{ker}_{1} x\right] / 2^{\frac{1}{2}} .
\end{array}
$$


SAVIDGE 1, and RusSELL, used the abbreviations

$$
\begin{aligned}
& X=X(x)=\operatorname{ber}^{2} x+\operatorname{bei}^{2} x, \quad V=V(x)=\operatorname{ber}^{\prime 2} x+\text { bei }^{\prime 2} x \\
& W=W(x)=\operatorname{ber} x \text { bei }^{\prime} x-\text { bei } x \operatorname{ber}^{\prime} x \text {, } \\
& Z=Z(x)=\operatorname{ber} x \operatorname{ber}^{\prime} x+\text { bei } x \text { bei }^{\prime} x \text {. }
\end{aligned}
$$

For the corresponding combinations of the ker and kei functions a similar notation with the suffix 1 was used. This notation was modified by CosTELLO ${ }^{3}$ into

$$
\begin{aligned}
X^{(k)}(x) & =\operatorname{ker}^{2} x+\operatorname{kei}^{2} x, \quad V^{(k)}(x)=\operatorname{ker}^{\prime 2} x+\operatorname{kei}^{\prime 2} x, \\
W^{(k)}(x) & =\operatorname{ker} x \operatorname{kei}^{\prime} x-\operatorname{kei} x \operatorname{ker}^{\prime} x \\
Z^{(k)}(x) & =\operatorname{ker} x \operatorname{ker}^{\prime} x+\text { kei } x \operatorname{kei}^{\prime} x
\end{aligned}
$$

and for the mixed functions for which SAvidge uses letters other than $X$, $W, V, Z$, Costello writes

$X^{(r)}(x)=$ ber $x \operatorname{ker} x+$ bei $x$ kei $x, \quad X^{(u)}(x)=$ bei $x$ ker $x-$ ber $x$ kei $x$ $V^{(r)}(x)=\operatorname{ber}^{\prime} x \operatorname{ker}^{\prime} x+\operatorname{bei}^{\prime} x \operatorname{kei}^{\prime} x, \quad V^{(u)}(x)=\operatorname{bei}^{\prime} x \operatorname{ker}^{\prime} x-\operatorname{ber}^{\prime} x \operatorname{kei}^{\prime} x$

while Brasey writes $B(x)$ for $x Z(x)$. Polar forms of the functions have been used by some writers, thus the notation $M_{n}(x) e^{i \theta_{n}(x)}$ above has been employed by Kennelly, Laws \& Pierce 1, and the corresponding notation $N_{n}(x) e^{i \phi_{n}(x)}$ by MCLACHLAN $\&$ MEYERS.

The four functions which B. A. SмIтн 3 calls Michell functions in honor of J. H. MicheLL who interested him in the tabulation of Bessel functions of the second kind, occurred first in the work of SмITH on arched dams and then later in the work of HolLISTER on hemispherical shells having tapered edges. The general solution of the differential equation which here comes up,

$$
D_{x}^{2}\left(x^{3} D_{x}^{2} u\right)+x u=0
$$

will be written in the form $u=A m_{1}+B m_{2}+C m_{3}+D m_{4}$, where

$$
\begin{aligned}
& m_{1}=m_{1}(x)=z^{-1} J_{1}(z)+w^{-1} J_{1}(w), z=2(i x)^{\frac{3}{3}}, w=2(-i x)^{\frac{1}{3}}, \\
& m_{2}=m_{2}(x)=2 i\left[z^{-1} J_{1}(z)-w^{-1} J_{1}(w)\right], \\
& m_{3}=m_{3}(x)=-2(\ln 2-1) m_{1}-\frac{1}{4} \pi m_{2}+2\left[z^{-1} Y^{(1)}(z)+w^{-1} Y^{(1)}(w)\right], \\
& m_{4}=m_{4}(x)=-\frac{1}{2} \pi m_{1}+(\ln 2) m_{2}-2 i\left[z^{-1} Y^{(1)}(z)-w^{-1} Y^{(1)}(w)\right] ; \\
& m_{1}=x^{-\frac{1}{3}} \text { bei }^{\prime}\left(2 x^{3}\right), m_{2}=-2 x^{-\frac{1}{3}} \operatorname{ber}^{\prime}\left(2 x^{3}\right) \text { (see also p. 308), }
\end{aligned}
$$

and $Y^{(1)}(z)$ is Neumann's Bessel function of the second kind.

In Tölke's tables the following notation is used

$$
\begin{aligned}
J_{p}\left(x i^{1}\right) & =J_{p 1}(x)+i J_{p 2}(x), \\
H_{p}^{(1)}\left(x i^{1}\right) & =H_{p 1}^{(1)}(x)+i H_{p 2}^{(1)}(x), \\
G_{p 1}(x) & =\frac{1}{2} \pi H_{p 2}^{(1)}(x), \quad G_{p 2}(x)=\frac{1}{2} \pi H_{p 1}^{(1)}(x) .
\end{aligned}
$$

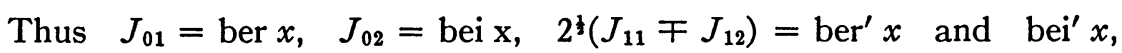
$J_{21}=\operatorname{ber}_{2} x, J_{22}=\operatorname{bei}_{2} x,\left(J_{31} \mp J_{32}\right)=-\left(\operatorname{ber}_{3} x \pm\right.$ bei $\left._{3} x\right) ; G_{01}=\operatorname{ker} x$, $G_{02}=\operatorname{kei} x, 2^{-\mathrm{z}}\left(G_{11} \mp G_{12}\right)=\operatorname{ker}^{\prime} x$ and $\operatorname{kei}^{\prime} x, G_{21}=\operatorname{ker}_{2} x, G_{22}=\operatorname{kei}_{2} x$, $\left(G_{31} \mp G_{32}\right)=-\left(\operatorname{ker}_{3} x \pm \operatorname{kei}_{3} x\right)$. 
In tables of Dubois the following notation is employed

Hence,

$$
\begin{gathered}
J_{2}\left[2(i x)^{\dagger}\right]=-I_{1}(x)-i I_{2}(x), \quad \pi H_{2}^{(1)}\left[2(i x)^{\left.\frac{1}{1}\right]}=I_{4}(x)-i I_{3}(x),\right. \\
\pi H_{2}^{(2)}\left[2(-i x)^{\frac{1}{1}}\right]=I_{4}(x)+i I_{8}(x) .
\end{gathered}
$$

$$
J_{21}=-I_{1}, \quad J_{22}=-I_{2}, \quad G_{21}=\frac{1}{2} I_{3}, \quad G_{22}=\frac{1}{2} I_{4} .
$$

1 KELvin, "Ether, electricity and ponderable matter," So. Electrical Telegraph Engineers, J., v. 18, 1889, p. 4-37; also in Mathematical and Physical Papers, v. 3, London, Cambridge Univ. Press, 1890 , p. 484-515.

¿C. S. WhITEHEAD, "On a generalisation of the functions ber $x$, bei $x$, $\operatorname{ker} x$, kei $x$," Quart. J. Math., v. 42, 1911, p. 316-342.

${ }_{3}$ J. B. Costello, "Bessel product functions," Phil. Mag., s. 7, v. 21, 1936, p. 308-318.

$$
\text { A. } J_{0}(z), J_{1}(z), Y_{0}(z), Y_{1}(z), z=r e^{i \theta}
$$

1. $15 \mathrm{D}, J_{0}(z)=u_{0}(r, \theta)+i v_{0}(r, \theta), r=0(1) 10$, NYMTP $1, \theta=0\left(5^{\circ}\right) 90^{\circ}$

2. $10 \mathrm{D}, J_{0}(z), J_{1}(z)=u_{1}(r, \theta)+i v_{1}(r, \theta), r=0(.01) 10$, NYMTP $3, \theta=0\left(5^{\circ}\right) 90^{\circ}$

3. $10 \mathrm{D}, J_{0}(z), J_{1}(z), r=0(.2) 8$, DiNNIK $11, \theta=\frac{\pi}{16}\left(\frac{\pi}{16}\right) \frac{1}{2} \pi$; also for the same range $v_{0}$ and $u_{1}, \theta=\pi / 2-.001$

4. 10D, $Y_{0}(z)=U_{0}(r, \theta)+i V_{0}(r, \theta), Y_{1}(z)=U_{1}(r, \theta)+i V_{1}(r, \theta), r=0(.01) 10$, NYMTP $6, \theta=0\left(5^{\circ}\right) 90^{\circ}$

5. $8-15 \mathrm{D}$, first 18 derivatives with respect to $r$ of $J_{0}(z), J_{1}(z), r=0(.1) 10$, NYMTP 5 , $\theta=0\left(5^{\circ}\right) 90^{\circ}$

6. 8-15D, $S_{0}(z), S_{1}(z), z=r e^{i \theta}, r=0(.1) 10$, NYMTP $7, \theta=0\left(5^{\circ}\right) 90^{\circ}$

\section{$\mathrm{B}_{1}$, ber $x$ and bei $x$}

1. $21 \mathrm{D}$, ALDIS $2, x=0(.1) 6$

2. $15 \mathrm{D}, \mathrm{NYMTP} 1, x=0(1) 10$

3. $10 \mathrm{D}$, NYMTP $3, x=0(.01) 10$

4. 9D, A. G. WEBSTER, $x=0(.1) 10, \Delta^{7}$

5. $9 \mathrm{D}$, LODGE $2, x=0(.2) 6$

6. 6D, BrASEY, $x=0(.02) 4.6$

7. 5-6S, DwIGHT $1, x=1(1) 10$

8. 5D, PeDERSEN, $x=0(.2) 6$

9. $5 \mathrm{~S}$, DwIGHT $4,6, x=0(.1) 20$

10. 4-5S, JAHNKe \& EMDe $1_{2}$ (p. 296f, 308) - 16 (p. 246f, 258), $x=0(.01) 6(.1) 10, \Delta$ up to $x=6$

11. 5D; $4 \mathrm{D}$, SCHLEICHER, $x=0(.01) 1(.02) 2 ; 2.1(.1) 6$

12. $4 \mathrm{D}$, DINNIK $11, x=0(.2) 8$

13. $4 \mathrm{D}$, TÖLKE, $\left[J_{01}, J_{02}\right], x=0(.01) 21$

14. $4 \mathrm{D}$ or $4 \mathrm{~S}$, MCLACHLAN $2, x=0(.1) 10$

15. $4 \mathrm{D}$, BISACRE, $x=0(.2) 6$

16. $4 \mathrm{~S}$, SAVIDGE $1, x=1(1) 30$

17. $4 \mathrm{D} ; 5 \mathrm{~S}+$, Maclean $1_{2}, x=0(.5) 6 ; 8,10(5) 20,30$

18. 3D, W. E. Miller, $x=0(.5) 6(2) 10(5) 20$; also $\int_{0}^{p}$ ber $x d x, \int_{0}^{p}$ bei $x d x, p=3,8$

19. 5D, bei $x /$ ber $x$, TAKAYA, $x=.2(.2) 8$

20. 2D, bei $x /$ ber $x$, Piola $1, x=0(.5) 6(2) 10(5) 20$

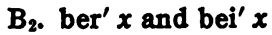

1. 9D, A. G. WeBster, $x=0(.1) 10$

2. 5D, PeDERSEN, $x=0(.2) 6$

3. $5 \mathrm{~S}$, Dwight $4,6, x=0(.1) 20$ 
4. 5D; $4 \mathrm{D}$, SCHLEICHER, $x=0(.01) 1(.02) 2 ; 2.1(.1) 6$

5. $4 \mathrm{D}$, TöLKE, $\left[2^{-1}\left(J_{11} \mp J_{12}\right)\right], x=0(.01) 21$

6. mostly $4 \mathrm{D}$, MaClean $1_{2}, x=0(.5) 6(2) 10(5) 20,30$

7. $4 \mathrm{D}, \mathrm{McLACHLAN} 2, x=0(.1) 10$

8. 3D, W. E. Miller, $x=0(.5) 6(2) 10(5) 20$

9. 3-5D, bei' $x$ /ber' $x$, SAKuRABA \& KimuRA, $x=.2(.2) 6$

B. $\operatorname{ber}_{n} x, \operatorname{bei}_{n} x, \operatorname{ber}_{n}^{\prime} x, \operatorname{bei}_{n}^{\prime} x$

1. 2-6S, ber $_{n} x$, bei $_{n} x, n=1$ (1)5, Dwight $1,2,3, x=1$ (1)10

2. $4 \mathrm{D}$ or $4 \mathrm{~S}, \operatorname{ber}_{n} x, \operatorname{bei}_{n} x, n=1(1) 5$, MCLACHLAN $_{2}, x=1(1) 10$

3. $4 \mathrm{~S}$, ber $_{2} x,-$ bei $_{2} x$, TöLkE, $x=0(.01) 21$

4. 4-9S, $I_{m}, m=1(1) 4$ (i.e., $-\operatorname{ber}_{2} x,-\operatorname{bei}_{2} x, 2 \operatorname{ker}_{2} x, 2 \operatorname{kei}_{2} x$ ), and their derivatives and integrals, DuBoIs, $x=.6,1.1,1.6, \cdots 38.5,42,45.5$, argument intervals irregular, for $m=1,2$ up to 45.5 . Since $I_{m}$ are functions practically tabulated by TöLKE, $r=2 x=.01(.01) 21, x=.000025, \cdots 110.25$ is the range of these tabulated to $4 \mathrm{D}$.

5. $10 \mathrm{D}$, ber $_{1} x$, bei $1 x$, NYMTP $1, x=0(.01) 10$

6. 5-6S, ber $_{1} x$, bei $1, x$, Dwight $1,2,3, x=1(1) 10$

7. $4 \mathrm{D}$, ber $_{1} x$, bei $_{1} x$, Dinnik $11, x=0(.2) 8$

8. $4 \mathrm{D}, \operatorname{ber}_{1} x$, bei $_{1} x$, MCLAChLAN $2, x=1(1) 10$

9. $4 \mathrm{D}$, ber $_{1} x$, bei $1 x$, BisACRE, $x=0(.2) 6$

10. $21 \mathrm{D},-2$ ber $_{1} x, 2$ bei $_{1} x$, Aldis $2, x=.1(.1) 6$

11. $6 \mathrm{D},-2 \operatorname{ber}_{1} x, \mathrm{bei}_{1} x$, BRASEY, $x=0(.02) 4.6$

12. $4 \mathrm{D},-2$ ber 1 , 2 bei $x$, JAhNKe \& EmDE $1_{1}$ (p. 138), $x=0(.1) 6$

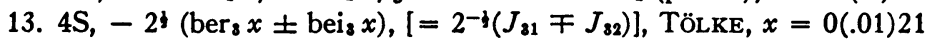

14. 2-6S, ber $_{n} x$, bei $_{n} x, n=1$ (1)5, Dwight 1, 2, 3, $x=1(1) 10$

15. $4 \mathrm{D}$ or $4 \mathrm{~S}$, ber $_{n}^{\prime} x$, bei $_{n} x, n=1(1) 5$, McLaCHLAN $2, x=1$ (1) 10

$$
\begin{aligned}
& \text { B. } \operatorname{ker} x=-(\pi / 2) \text { hei } x \text {, kei } x=(\pi / 2) \text { her } x, \operatorname{ker}^{\prime} x=-(\pi / 2) \text { hei' }^{\prime} \text {, }
\end{aligned}
$$

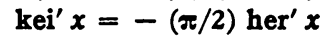

1. 7D; 8D; 9D, $\operatorname{ker} x$, kei $x, \operatorname{ker}^{\prime} x, \mathrm{kei}^{\prime} x$, SAVIDGE $2, x=0(.1) 3.8 ; 3.9(.1) 6.4 ; 6.5(.1) 10$

2. $4 \mathrm{~S}, \operatorname{ker} x\left[=G_{01}\right]$, $\operatorname{kei} x\left[=G_{02}\right], \operatorname{ker}^{\prime} x\left[=2^{\prime}\left(G_{11}-G_{12}\right)\right], \operatorname{kei}^{\prime} x\left[=2^{-i}\left(G_{11}+G_{12}\right)\right]$, TöLKE, $x=0(.01) 21$

3. 3-4S, $\operatorname{ker} x$, kei $x, \operatorname{ker}^{\prime} x, \operatorname{kei}^{\prime} x$, MCLAChLAN $2, x=0(.1) 10$

4. $4 \mathrm{~S}$, $\operatorname{ker} x$, kei $x$, SAvidge $1, x=1(1) 30$

5. 5D, kei $x / \operatorname{ker} x$, TAKAYA, $x=.2(.2) 8$

6. 3-5D, kei' $x / \operatorname{ker}^{\prime} x$, SAKURABA \& KIMURA, $x=.2(.2) 6$

7. 5D, - her $x$, hei $x\left(Z_{3}, Z_{4}\right)$, SCHLEICHER, $x=0(.01) 1(.02) 2(.2) 6$

8. $4 \mathrm{~S}$, - her $x$, - hei $x$, BISACRE, $x=0(.2) 6$

9. 3-4S, - her $x$, - hei $x$, JAHNKE \& EMDE $1_{2}$ (p. 302f) - $1_{6}$ (p. 252f), $x=0(.01) 6(.1) 10$

10. $5 \mathrm{D},-\operatorname{her}^{\prime} x$, hei' $x$, SChLEICHER, $x=0(.01) 1(.02) 2(.2) 6$

11. 3-5S, $-\operatorname{her}^{\prime} x$, hei' $x$, JAHNkE \& EMDE $1_{2}$ (p. 304f) - $1_{5}$ (p. 254f), $x=0(.01) 6(.1) 10$

\section{B. $\operatorname{ker}_{n} x, \operatorname{kei}_{n} x, \operatorname{ker}_{n}^{\prime} x, \operatorname{kei}_{n}^{\prime} x, \operatorname{her}_{n} x, \operatorname{hei}_{n}^{\prime}(x), \operatorname{yer}_{n} x, \operatorname{yei}_{n} x$}

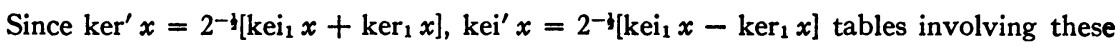
functions when $n=1$ are given in $B_{4}$.

1. 4-6S, $\operatorname{ker}_{n} x, \operatorname{kei}_{n} x, \operatorname{ker}_{n}^{\prime} x, \operatorname{kei}_{n}{ }_{n} x, n=1(1) 5$, Dwighr $3, x=1(1) 10$

2. $4 \mathrm{~S}, \operatorname{ker}_{n} x, \operatorname{kei}_{n} x, \operatorname{ker}_{n} x, \operatorname{kei}_{n} x, n=1(1) 5, \mathrm{MCLACHLAN} 2, x=1$ (1) 10

3. $4 \mathrm{~S}, \operatorname{ker}_{2} x, \operatorname{kei}_{2} x,-2^{-i}\left(\operatorname{ker}_{3} x \pm \operatorname{kei}_{3} x\right)=\left[2^{-1}\left(G_{31} \pm G_{32}\right)\right]$, TöLkE, p. 19f, $x=0(.01) 21$

4. 5-6S, her $1 x$, hei 1 , JAHNKE \& EMDE $1_{1}$, p. $140, x=0(.2) 6$

5. $4 \mathrm{~S}$, her 1 , hei, $x$, Bisacre, $x=0(.2) 6$

6. 5D, yer $1 x=-\operatorname{bei}_{1} x-(2 / \pi) \operatorname{ker}_{1} x$, yei $_{1} x=\operatorname{ber}_{1} x-(2 / \pi) \operatorname{kei}_{1} x$, KOHLER, $x=0(.2) 6$; graphs

7. $10 \mathrm{D}, Y_{1}\left(x i^{i}\right)=-\left(\operatorname{yer}_{1} x+2\right.$ bei $\left._{1} x\right)+i\left(\operatorname{yei}_{1} x-2\right.$ ber $\left._{1} x\right)$, NYMTP $6, x=0(.01) 10$ 


\section{Michell and Airey functions}

1. 9D, $m_{n}(x), m_{n}{ }^{\prime}(x), m_{n}{ }^{\prime \prime}(x), m_{n}{ }^{\prime \prime \prime}(x), n=1(1) 4$, HAwKINS, TOBEY \& LEE, $x=0(.5) 10(1) 30$

2. $8 \mathrm{D}, m_{n}(x), m_{n}^{\prime}(x), m_{n}{ }^{\prime \prime}(x), m_{n}^{\prime \prime \prime}(x), n=1(1) 4$, B. A. SмITH $3, x=0(1) 30$, and $.5,1.5$

3. $8 \mathrm{D}, I_{0}^{\prime}\left(x i^{i}\right) \cdot G_{0}{ }^{\prime}\left(x i^{i}\right)=V_{r}(x)+i V_{u}(x)$, AIREY 34 ,

$x=0(.02) 10(.05) 11(.1) 12.5(.5) 16(1) 20$

\section{D. $X, V, W, Z, X^{(k)}, V^{(k)}, W^{(k)}, Z^{(k)}, V^{(r)}, V^{(u)}$}

1. 6S, $W, V, W, Z, X^{(k)}, V^{(k)}, W^{(k)}, V^{(r)}, V^{(u)}$, SAvidge $3, x=0(.2) 10$

2. $6 \mathrm{D}, X, x Z$, BRASEY, $x=0(.02) 4.6$

3. 2-6D, $X, V, W, Z$, Pedersen, $x=0(.2) 6$

4. $4 \mathrm{~S}, X, V, W, Z, X^{(k)}, V^{(k)}, V^{(r)}, V^{(u)}$, SAVIDGE $1, x=0(1) 30$

5. $4 \mathrm{~S}, W^{(k)}, Z^{(k)}$, SAVIDGE $1, x=0(1) 5$

6. $2 \mathrm{D}, X^{i}$, Piola $1, x=0(.5) 6(2) 10(5) 20$

$$
\mathrm{D}_{2} \cdot \frac{V}{X}, \frac{W}{X}, \frac{Z}{X}, \frac{Z}{V}, \frac{W}{V}, \text { etc. }
$$

1. 6-5D, $V / X, W / X, Z / X$, SAvidge $3, x=0(.2) 10, \infty$

2. $6 \mathrm{D}, W / X, Z / X$, BuTterWORTh $1, x=3(1) 6$

3. $4 \mathrm{D}, V / X, W / X, Z / X$, SAVIDGE $1, x=0(1) 30, \infty$

4. $4 \mathrm{D}, Z / X$, RUSSELL, $x=3(.2) 7$

5. $4-5 \mathrm{D}, 2 Z /(x X), 2 W /(x X)$, W. FISCHER, $x=0(.5) 6(2$ or 5$) 20$

6. $5 \mathrm{D}, 2 Z / x X, 2 W / x X$, Piola $2, x=0(.5) 6(2) 10,15, \infty$; also graphs of the functions $0<x<11$

7. $3-4 \mathrm{D}, \frac{1}{2} x Z / V, \frac{1}{2} x W / V$, MACLEAN \& ZENNECK, $x=.5(.5) 6(2) 10(5) 20$; also $x / 2^{3 / 2}=[0(.1) .9 ; 2-3 \mathrm{D}]$

8. $4 \mathrm{~S}$ and $3 \mathrm{~S}, \psi_{1}(x)=2 Z /(x X), G=x Z /(4 X), \psi_{2}=4 W /(x V)-8 / x^{2}, F=x W /(2 V)-1$, Butterworth $2, x=0(.5) 5$; also $\psi_{1} / x^{2}$ to $4 \mathrm{D}$, and $\psi_{2} / x^{2}$, to $5 \mathrm{D}, x=0(.5) 1(1) 3$. Graphs of $\psi_{1}, \psi_{2}, F_{1}, G, 0<x<5$

9. $6 \mathrm{D}, x Z /(2 V), x W /(2 V)$, ALGER, $x=0(.1) 10, \Delta^{4}$

10. $6 \mathrm{D}, Z / V, W / V$, BUTTERWORTH $1, x=5(1) 8$

11. 5D, $Z / V, W / V, \frac{1}{2} x W / V, 4 Z / x V$, Rosa \& GROVER, $x=0(.1) 5(.2) 10(.5) 15(1) 26(2) 50(10) 100, \infty, \Delta^{2}$

12. $5 \mathrm{D}, Z / V, W / V$, SAVIDGE $3, x=0(.2) 10, \infty$

13. $4 \mathrm{D}, Z / V, W / V$, SAVIDGE $1, x=0(1) 30, \infty$

14. $4 \mathrm{D}, W / V, \frac{1}{2} x W / V$, Maclean, also $W / V$, LeVASSEUR, and $\frac{1}{2} x W / V$, Fleming, $x=0(.5) 6(2) 10(5) 20,30$

15. $3 \mathrm{D}, W / V, \frac{1}{2} x W / V$, W. E. MiLleR, $x=0(.5) 6(2) 10(5) 20$

16. $4 \mathrm{D}, W / V$, RUSSELL, $x=.5(.5) 2,5.5,6(2) 10(5) 20(10) 50$

17. 4S; 3D, ber' $x / V$, bei' $x / V ; Z / V, W / V$, WeINREICH, $x=0(.5) 6(2) 10(5) 20$

18. $5 \mathrm{~S}, W^{(k)} / Z^{(k)}, W / Z$, SakURABa $\&$ KimURA, $x=.2(.2) 4(1) 10$

19. $5 \mathrm{D}, Z^{(k)} / W^{(k)}$, TAKAYA, $x=.2(.2) 2.6,3.2(.2) 4(1) 10$

20. $5 \mathrm{D}, Z / W$, '

21. 2-3D, $x Z / V, x W / V$, Thомson, $x^{2}=.5(.5) 3$

22. 2D, $-Z / W,(2 / x)(V / X)^{3}$, Piola $1, x=0(1) 6(2) 10,20$

$$
\begin{gathered}
\text { E. } M_{n}(x)=\left(\operatorname{ber}_{n}^{2} x+\operatorname{bei}_{n}^{2} x\right)^{13}, \quad \theta_{n}(x)=\tan ^{-1}\left(\operatorname{bei}_{n} x / \operatorname{ber}_{n} x\right), \\
N_{n}(x)=\left(\operatorname{ker}_{n}^{2} x+\operatorname{kei}_{n}^{2} x\right)^{3}, \quad \phi_{n}(x)=\tan ^{-1}\left(\operatorname{kei}_{n} x / \operatorname{ker}_{n} x\right), \quad n=0,1
\end{gathered}
$$

1. 6-5D; $0^{\circ} .00001, M_{0}(x), M_{1}(x) ; \theta_{0}(x), \theta_{1}(x)-180^{\circ}$, ALGER, p. $295, x=0(.1) 10$

2. $6 \mathrm{D} ; 0^{\circ} .00001,(X / V)^{3}=\frac{1}{2} x M_{0} / M_{1} ; \tan ^{-1}(Z / W)=\theta_{0}-\theta_{1}+135^{\circ}$, ALGER, p. 296f, $x=0(.1) 10$ 
3. $5 \mathrm{~S} ; 0^{\circ} .001, M_{0}(x), M_{1}(x) ; \theta_{0}(x), \theta_{1}(x)-45^{\circ}$, Dwight $4,6, x=0(.1) 20$

4. 5S; $0^{\circ} .001, M_{0}(x), M_{1}(x) ; \theta_{0}(x), \theta_{1}(x)-180^{\circ}$, Kennelly, Laws \& Pierce, $x=0(.1) 10$. "Figs. 21-24 give curves corresponding to entries in the table whereby interpolation may be made by direct inspection for most engineering purposes."

5. $5 \mathrm{~S} ; 1^{\prime \prime}, M_{0}(x), M_{1}(x) ; \theta_{0}(x), \theta_{1}(x)-180^{\circ}$, BISACRE, $x=0(.2) 6$

6. 4-5S; 08.01, $M_{0}(x), M_{1}(x) ; \theta_{0}(x), 200^{8}-\theta_{1}(x)$, JAHNKE $\&$ EMDE $1_{2}$ (p. 312) - $1_{5}$ (p. 262), $x=0(.1) 10$

7. $4 \mathrm{~S} ; 0^{\circ} .01, M_{0}(x) ; \theta_{0}(x), \log \left[x M_{0}(x)\right]$, McLachlan 2 , $x=0(.05) 2(.1) 4(.5) 6(1) 12(2) 20(5) 45$

8. $4 \mathrm{~S} ; 0^{\circ} .01, M_{1}(x) ; \theta_{1}(x), \log \left[x M_{1}(x)\right]$, McLACHLAN 2 , $x=0(.05) 2.7(.1) 3.8,4.25,4(.5) 8(1) 12(2) 20(5) 50$

9. $4 \mathrm{D}, 0^{\mathrm{r}} .0001, \ln \left[(2 \pi x)^{3} M_{0}(x)\right] ; \theta_{0}(x)$, SAvIDGE $1, x=1(1) 30$

10. $5 \mathrm{~S} ; 0^{\circ} .001, N_{0}(x), N_{1}(x) ; \phi_{0}(x), \phi_{1}(x)$, MCLACHLAN \& MEyers, $x=0(.1) 10$

11. $4 \mathrm{~S} ; 0^{\mathrm{r}} .0001, \ln \left[(2 x / \pi) N_{0}(x)\right] ; \phi_{0}(x)$, SAvidge $1, x=1(1) 30$

12. $4 \mathrm{~S} ; 1^{\prime \prime},(2 / \pi) N_{0}(x), N_{1}(x) ; \phi_{0}(x)+90^{\circ}, \phi_{1}(x)+90^{\circ}$, BISACRE, $x=0(.2) 6$

13. 4-5S; 08.01, $(2 / \pi) N_{n}(x) ;-\phi_{n}(x)-100, n=0,1$, JAHNKE \& EMdE $1_{2}$ (p. 312 ) -1 。 (p. 262)

14. 4-5S; 08.01, $(X / V)^{\frac{1}{3}}=M_{0} / M_{1}, \frac{1}{2} x M_{0} / M_{1}, 2 M_{0} /\left(x M_{1}\right),\left(X^{(k)} / V^{k}\right)^{t}=N_{0} / N_{1}, \frac{1}{2} x N_{0} / N_{1}$, $2 N_{0} / x N_{1} ; \theta_{1}-\theta_{0}-200 \mathrm{~g}, \phi_{1}, \phi_{0}$, JAHNke \& EMDE $1_{2}$ (p. 316f, figs. 165-166), $1_{s}-1_{5}$ (p. 266f, figs. 144-145). Graphs

15. $2 \mathrm{D}, M_{0}(x) ; \tan \theta_{0}(x)$, Piola $1, x=0(.5) 6(2) 10(5) 20$

\section{F. Miscellaneous}

1. $J_{n}^{\prime}(x-i x) / J_{n}(x-i x), n=1(1) 3$, SeITZ 1, p. 753-754, $x=480$

2. $5 \mathrm{D} ; J_{n}(x-i x), J_{n}^{\prime}(x-i x), n=0,1,2, \operatorname{SEITZ} 1$, p. 758-9, $x=.3302$

3. $4 \mathrm{D},\left|i J_{1}(z) /\left[z J_{0}(z)\right]\right|$, also real parts of this function, Buchнolz 1 , tables for $|z|=.6(.1) 2$, and graphs

4. 3D, SchirmanN, table of complex nos. $a_{1}, a_{2}, p_{1}$ for $\lambda=500(50) 650,2 \rho \cdot 10^{8} \mathrm{~cm}=$ (i) 20.7, (ii) 16.8 , where $a_{v}=\left[(2 v+1) / 2 \alpha^{3}\right]\left[I_{v}{ }^{\prime}(\alpha) I_{v}(\beta) \beta-I_{v}{ }^{\prime}(\beta) I_{v}(\alpha) \alpha\right] /\left[K_{v}{ }^{\prime}(-\alpha) I_{v}(\beta) \beta\right.$ $\left.-I_{v}^{\prime}(\beta) K_{v}(-\alpha) \alpha\right]$ and $p_{v}=\left[-(2 v+1) / 2 \alpha^{3}\right]\left[I_{v}(\alpha) I_{v}^{\prime}(\beta) \beta-I_{v}(\beta) I_{v}^{\prime}(\alpha) \alpha\right] /$

$\left[K_{v}(-\alpha) I_{v}^{\prime}(\beta) \beta-I_{v}(\beta) K_{v}^{\prime}(-\alpha) \alpha\right], I_{v}(x)=\left(\frac{1}{2} \pi x\right) J_{v+1}(x), K_{v}(-x)=\left(\frac{1}{2} \pi x\right)^{1} H_{v+1}^{2}(x)$. $\alpha=2 \pi m_{0} \rho / \lambda, \beta=2 \pi m \rho / \lambda$, and when $\lambda=500, m=1.10-2.02 i ; \lambda=550$,

$m=.57-2.45 i ; \beta=600, m=.38-2.96 i ; \lambda=650, m=.41-3.54 i ; m_{0}=1$

\section{G. Zeros}

1. $5 \mathrm{D}$, ber $x$, bei $x$, ber $x$, bei' $x$, ker $x$, kei $x, \operatorname{ker}^{\prime} x$, $\operatorname{kei}^{\prime} x$, ArRey 29 , first ten zeros

2. $3 \mathrm{D}, X Z-X(x V-Z)+2 x Z^{2}$, STrutt 2, a zero, $x=2.514$

3. $4 \mathrm{D}, H_{1}^{(2) '}(z)$ or $H_{0}^{(2)}(z)-z^{-1} H_{1}^{(2)}(z)$, JAHNKE \& EMDE $1_{1}$ (p. 142), $1_{3}-1_{5}$ (p. 242), a zero, $z=r e^{i \theta}=0.5012+i 0.6435, r=0.8156, \theta=57 \mathrm{~g} .87=52^{\circ} .085=0^{\mathrm{r}} .9091$

4. Chart for zeros of $H_{n}^{(1)}(z), H_{n}^{(2)}(z), z=x+i y,|x|<4,-3<y<+4$, EMDE \& Rühle, and Jahnke \& EMde $1_{2}$ (fig. 150), $1_{3}-1_{5}$ (fig. 129)

5. Charts for complex zeros of $J_{-n}(x+i y)$, JAHNKE \& EMDE $1_{3}-1_{5}$ (figs. 122-125)

\section{H. Graphs and reliefs}

1. $J_{0}(z)=J_{0}\left(\rho e^{i \phi}\right)=U(\rho, \phi)+i V(\rho, \phi)$, NYMTP 1 , contour lines $U(\rho, \phi)=$ constant, $V(\rho, \phi)=$ constant

2. $J_{0}(z)=R e^{i \theta}$, NYMTP 1, contour lines $\theta=\theta(\rho, \phi)=$ constant, $R=R(\rho, \phi)=$ constant

3. $J_{1}(z)=u(\rho, \phi)+i v(\rho, \phi)$, NYMTP 1 , contour lines $u(\rho, \phi)=$ constant, $v(\rho, \phi)=$ constant

4. $J_{1}(z)=S e^{i \sigma}$, NYMTP 1, contour lines $\sigma=\sigma(\rho, \phi)=$ constant, $S=S(\rho, \phi)=$ constant 
5. $J_{0}(z)=J_{0}(x+i y)$, JAHNKE \& EMDE $1_{2}$ (p. 193) - $1_{5}$ (p. 127), altitude chart, $x=0(.5) 7$; $y=-2(.5)+2$

6. $J_{0}(x i$ ) $), J_{1}(x i$ ) $)$ JAHNKe \& EMDE $1_{2}$ (p. 294-295) - 1s (p. 244-245), $1_{1}$ (p. 136-137), $0<x<10$

7. $Y_{0}\left[x( \pm i)^{i}\right], Y_{\mathrm{l}}\left[x( \pm i)^{i}\right]$, SCHLEICHER, p. $127,0<x<6$

8. $-J_{0}(z) /\left[z J_{1}(z)\right], Z_{E N N E C K}, 4$ figures

9. $-R\left\{J_{0}(z) /\left[z J_{1}(z)\right]\right\}$, where $z=x(2 i)^{\xi}$, KADEN \& KAUFMANN, $x=.1(.1) 1(1) 10(10) 100$

10. ber $x$, bei $x$, ber' $x$, bei' $x$, ber $2 x$, bei $2 x$, ber ${ }_{3} x \pm$ bei $_{3} x$, TöLKE, p. $7,0<x<12$

11. ber $x$, bei $x$, ber' $x$, bei' $x$, SCHLEICHER, p. $123,0<x<6$

12. ber $x$, bei $x$, MCLACHLAN 2, p. $120,0 \leq x \leq 7$

13. $\operatorname{ker} x, \operatorname{kei} x, \operatorname{ker}^{\prime} x, \operatorname{kei}^{\prime} x, \operatorname{ker}_{2} x, \operatorname{kei}_{2} x, \operatorname{ker}_{3} x \pm \operatorname{kei}_{3} x$, TölkE, p. $12,0<x<8$

14. $-\operatorname{ber}_{2} x,-\operatorname{bei}_{2} x, 2 \operatorname{ker}_{2} x, 2 \operatorname{kei}_{2} x$ and their derivatives and integrals, Dubors, $0<x<35$; see XI B 4

15. hèr $x$, hei $x$, her' $x$, hei' $x$, Schleicher, p. $130,0<x<6$

16. $W / X, Z / X$, WRIGHT, $0<x<12$

17. $2 Z / x X$, BURCH $\&$ Davis, $0<x<10$

18. $x Z / X, Z / x X$, STRUTt $1,0<x<10$

19. $\frac{1}{2} x W / V, \frac{1}{2} x Z / V$, SChelkUNOFF, p. $265,0<x<12$

20. (a) $\frac{1}{2} x W / V, 2 Z /(x V)$; (b) ber $x / X$, W. E. MilleR, (a), $0<x<20$; (b) $0<x<16$

21. $H_{3.6}^{(1)}(2)$, altitude chart, JAHNKE \& EMDE $1_{2}$ (fig. 97) $-1_{5}$ (fig. 72), $z=x+i y,|x| \leq 5$, $|y| \leq 3$

22. $H_{3.5}^{(1)}(z)$, relief, JAHNKe \& EMdE $1_{2}$ (fig. 98) - $1_{5}$ (fig. 73)

23. $H_{0}(1)(z)$, relief, branch line along the imaginary axis, $z=x+i y=r e^{i \theta}$, $-90^{\circ}<\theta<270^{\circ}$, JAHNKe \& EMDE 12 (fig. 99) $-1_{5}$ (fig. 74), $|x| \leq 3,|y| \leq 2$

24. $H_{0}{ }^{(1)}(z), H_{0}^{(2)}(z)$, altitude chart, JAHNKE \& EMDE $1_{2}$ (fig. 100) - $1_{5}$ (fig. 75), $x=-4(.5)+2, y=-1.5(.5)+1.5$

25. $H_{0}^{(1)}(z)$, relief, JAHNKE \& EMDE $1_{2}$ (fig. 101) - $1_{5}$ (fig. 76), $x=-5(.25)+2.5$, $y=-2(.05)+1.4$

26. $H_{n}{ }^{(1)}\left(x i^{i}\right), n=0,1$, JAhNKe \& EMde $1_{2}$ (figs. 155-158) $-1_{5}$ (figs. 134-137), $0<x<10$

27. $H_{n}{ }^{(1)}\left(r i^{i}\right)=h i^{\eta}, n=0,1$ JAHNKE \& EMDE $1_{2}$ (figs. 159-160)-1 $1_{5}$ (figs. 138-139), $h$ and $\eta$ against $r, 0<r<10$

28. $H_{0}^{(1)}\left(r i^{3}\right) / H_{1}^{(1)}\left(r i^{3}\right)$, JAHNKE \& EMDE $1_{2}$ (fig. 166) $-1_{6}$ (fig. 145), $0<r<10$

29. $H_{n}{ }^{(1)}\left(r i^{i}\right)=h \imath^{\eta}$, the arrows going out perpendicularly from the $r$-axis, JAHNKE \& EMDE $1_{2}$ (figs. 167-168) - $1_{5}$ (figs. 146-147); compare XI E 12

30. Magnitude and phase of $H_{0}^{(1)}\left(x i^{i}\right)$, and $i^{3} H_{1}^{(1)}\left(x i^{i}\right)$, RÜDENBURG, $x=0(.5) 6$

31. Vector diagrams of $H_{0}^{(1)}\left(x i^{i}\right)$ and $i^{i} H_{1}{ }^{(1)}\left(x i^{i}\right)$, RüDENBURG

32. $(\pi / 10) H_{0}^{(1)}\left(x i^{3}\right)$, RÜDENBURG, $1230 x=5,10,20,50,100,200,1000,2000,5000$

33. $J_{n}\left(r i^{i}\right)=b i^{\beta}, n=0,1$, JAHNKE \& EMDE $1_{2}$ (figs. 161-164) - $1_{5}$ (figs. 140-143); see XI E 5

34. $J_{0}\left(r i^{3}\right) / J_{1}\left(r i^{3}\right)$, JAHNKE \& EMDE $1_{2}$ (fig. 165) - $1_{5}$ (fig. 144)

See also XI: $B_{3} 4 ; B_{6} 6 ; D_{2} 6,8 ; E 4,14 ; F 3 ; G 4,5$

\section{Series of Bessel Functions; Lommel}

FUNCTIONS $U_{n}(y, z), V_{n}(y, z)$

In his papers of 1886 Lommel 2, 3 introduced the functions

$$
\begin{aligned}
& U_{n}(y, z)=\sum_{s=0}^{\infty}(-1)^{s}(y / z)^{n+2 s} J_{n+2 s}(z) \\
& V_{n}(y, z)=\sum_{s=0}^{\infty}(-1)^{s}(y / z)^{-n-2 s} J_{-n-2 s}(z)
\end{aligned}
$$


and showed among other things that they had the representations

$$
\begin{aligned}
U_{n}(y, z) & =y^{n} z^{1-2 n} \int_{0}^{1}(z u)^{n} J_{n-1}(z u) \cos \left[\frac{1}{2} y\left(1-u^{2}\right)\right] d u \\
U_{n+1}(y, z) & =y^{n} z^{1-2 n} \int_{0}^{1}(z u)^{n} J_{n-1}(z u) \sin \left[\frac{1}{2} y\left(1-u^{2}\right)\right] d u \\
-V_{n+1}(y, z) & =y^{1-n} z^{2 n-1} \int_{1}^{\infty}(z u)^{1-n} J_{-n}(z u) \cos \left[\frac{1}{2} y\left(1-u^{2}\right)\right] d u \\
V_{n}(y, z) & =y^{1-n} z^{2 n-1} \int_{1}^{\infty}(z u)^{1-n} J_{-n}(z u) \sin \left[\frac{1}{2} y\left(u^{2}-1\right)\right] d u .
\end{aligned}
$$

The functions are not really independent, for LommeL 3, p. 559, obtained the relation

$$
V_{2-n}(y, z)=U_{n}(y, z)-\cos \left(\frac{1}{2} y+z^{2} / 2 y-\frac{1}{2} n \pi\right)
$$

which may be used to define $V_{m}$ for any value of $m$. For large and almost equal values of $y$ and $z$ MAYALL has indicated a suitable method of computation. When $z=0$ there is a simplification, and LOMMEL 3, p. 582, gives the relations

$$
\begin{aligned}
U_{n}(y, 0) & =[2 / \Gamma(n)]\left(\frac{1}{2} y\right)^{n} \int_{0}^{1} u^{2 n-1} \cos \left[\frac{1}{2} y\left(1-u^{2}\right)\right] d u \\
U_{n+1}(y, 0) & =[2 / \Gamma(n)]\left(\frac{1}{2} y\right)^{n} \int_{0}^{1} u^{2 n-1} \sin \left[\frac{1}{2} y\left(1-u^{2}\right)\right] d u .
\end{aligned}
$$

The integrals on the right depend on the Fresnel functions when $n=\frac{1}{2}$. For the functions $V_{n}(y, 0)$ the analogous relations obtained by LOMMEL 3 , p. 583 , are

$$
\begin{array}{r}
V_{m+n}(y, 0)=(-1)^{m} 2^{n} y^{1-m-n} k_{m, n} \int_{1}^{\infty} u^{1-2 m-2 n} \sin \left[\frac{1}{2} y\left(u^{2}-1\right)\right] d u \\
V_{m+n+1}(y, 0)=(-1)^{m+1} 2^{n} y^{1-m-n} h_{m, n} \int_{1}^{\infty} u^{1-2 m-2 n} \cos \left[\frac{1}{2} y\left(u^{2}-1\right)\right] d u
\end{array}
$$

where

$$
k_{m, n}(-n) !=(2 n)^{m / 2}=h_{m, n}(-n) !
$$

Lommel 3, p. 591, followed Gilbert (1863) in expressing the Fresnel integrals (compare Section $\mathrm{X}$ ) in the form

$$
\begin{aligned}
C(2 z / \pi)^{\frac{1}{3}}=\frac{1}{2} \int_{0}^{z} J_{-\frac{3}{3}}(t) d t & =2^{-\frac{1}{3}}\left[\cos z U_{3}(2 z, 0)+\sin z U_{3 / 2}(2 z, 0)\right] \\
& =\frac{1}{2}+2^{-\frac{1}{3}}\left[\sin z V_{3}(2 z, 0)+\cos z V_{3 / 2}(2 z, 0)\right] \\
S(2 z / \pi)^{\frac{3}{2}}=\frac{1}{2} \int_{0}^{z} J_{\frac{1}{3}}(t) d t & =2^{-\frac{1}{3}}\left[\sin z U_{3}(2 z, 0)-\cos z U_{3 / 2}(2 z, 0)\right] \\
& =\frac{1}{2}-2^{-\frac{1}{3}}\left[\cos z V_{3}(2 z, 0)-\sin z V_{3 / 2}(2 z, 0)\right]
\end{aligned}
$$


where, for example,

$$
\begin{gathered}
V_{\mathfrak{z}}(2 z, 0)=(1 / \pi) \int_{0}^{\infty} e^{-u z} u^{-\frac{1}{3}} d u /\left(1+u^{2}\right), \\
V_{3 / 2}(2 z, 0)=-(1 / \pi) \int_{0}^{\infty} e^{-u z} u^{\frac{1}{3}} d u /\left(1+u^{2}\right) .
\end{gathered}
$$

These are merely particular cases of the more general integral

$$
V_{m+n}(2 z, 0)=(-1)^{m}(1 / \pi) \sin (n \pi) \int_{0}^{\infty} e^{-z u} u^{m+n-1} d u /\left(1+u^{2}\right)
$$

which readily gives the special values

also

$$
V_{n}(0,0)=\cos \left(\frac{1}{2} n \pi\right), \quad V_{n+1}(0,0)=-\sin \left(\frac{1}{2} n \pi\right) ;
$$

$$
U_{n}(y, 0)=V_{2-n}(y, 0)+\cos \left(\frac{1}{2} y-\frac{1}{2} n \pi\right) .
$$

LOMMEL 3, p. 592, gives the series

$$
\begin{aligned}
U_{i}(2 z, 0) & =(4 z / \pi)^{\frac{1}{3}}\left[1-\frac{(2 z)^{2}}{1.3 .5}+\frac{(2 z)^{4}}{1.3 .5 .7 .9}-\cdots\right] \\
U_{3 / 2}(2 z, 0) & =(4 z / \pi)^{\frac{1}{2}}\left[\frac{2 z}{1.3}-\frac{(2 z)^{3}}{1.3 .5 .7}+\frac{(2 z)^{5}}{1.3 .5 .7 .9 .11}-\cdots\right]
\end{aligned}
$$

which are well known in the theory of Fresnel's integrals. The asymptotic series of CAUCHY ${ }^{1}$ (1827) are written in the form

$$
\begin{aligned}
V_{3}(2 z, 0) & =(4 z / \pi)^{\frac{1}{3}}\left\{\frac{1}{(2 z)}-\frac{1.3}{(2 z)^{3}}+\frac{1.3 .5 .7}{(2 z)^{5}}-\cdots\right\} \\
V_{3 / 2}(2 z, 0) & =-(4 z / \pi)^{\frac{1}{3}}\left\{\frac{1}{(2 z)^{2}}-\frac{1.3 .5}{(2 z)^{4}}+\frac{1.3 .5 .7 .9}{(2 z)^{6}}-\cdots\right\} .
\end{aligned}
$$

LOMmel 3, p. 594, uses the notation of GILBERT

$$
M\left(x^{2}\right)=2^{-\frac{1}{2}} V_{3}\left(\pi x^{2}, 0\right), \quad N\left(x^{2}\right)=-2^{-\frac{1}{2}} V_{3 / 2}\left(\pi x^{2}, 0\right)
$$

who made a table for values of $x^{2}$. Struve 1,2 , on the other hand, used the following notation:

$$
N(v)=\frac{1}{2} \pi^{\frac{1}{3}} V_{\frac{1}{3}}\left(2 v^{2}, 0\right), \quad M(v)=-\frac{1}{2} \pi^{\frac{1}{3}} V_{3 / 2}\left(2 v^{2}, 0\right) .
$$

1 A. Cauchy, Oeuvres, s. 1, v. 1, Paris, 1887 , p. 277-278.

\section{A . Lommel functions of a single variable}

1. 5D, $M\left(m^{2}\right)=2^{-\mathrm{b}} V_{\mathrm{z}}\left(m^{2} \pi, 0\right), N\left(m^{2}\right)=-2^{-\mathrm{i}} V_{3 / 2}\left(m^{2} \pi, 0\right)$, GILBERT $m^{2}=0(.01) .38(.02) 2(.1) 9(1) 19,20.6,21.7,22(1) 30$

2. 4D, $M\left(m^{2}\right)=2^{-3} V_{4}\left(m^{2} \pi, 0\right), N\left(m^{2}\right)=-2^{-\frac{1}{2}} V_{3 / 2}\left(m^{2} \pi, 0\right)$, Miller \& GoRdON, $m=0(.05) 1.5(.1) 8.5(.5) 13$

3. 5D, $M(v)=-\frac{1}{2} \pi^{3} V_{3 / 2}\left(2 v^{2}, 0\right), N(v)=\frac{1}{2} \pi^{3} V_{3}\left(2 v^{2}, 0\right)$, STRUVE $1, v=0(.1) 6$

4. $6 \mathrm{D},(\pi / 2 x)^{3} V_{3}(y, 0),(\pi / 2 x)^{1} V_{3 / 2}(y, 0), M_{1}^{2}=(\pi / 2 x)\left[V_{1}^{2}(y, 0)+V_{3 / 2}^{2}(y, 0)\right]$, LOMMEL 3, T. XVI, p. $658, y=0(1) 60$ 
5. $6 \mathrm{D}, \frac{1}{2} V_{3}(x, 0), \frac{1}{2} V_{3 / 2}(x, 0), M_{2}^{2}=\frac{1}{4}\left[V_{1}^{2}(x, 0)+V_{3 / 2}^{2}(x, 0)\right]$, = (Liverpool Index) $\frac{1}{2}\left[\frac{1}{2}-C(x / 2 \pi)^{\frac{1}{2}}\right]^{2}+\frac{1}{2}\left[\frac{1}{2}-S(x / 2 \pi)^{\frac{1}{2}}\right]^{2}$, LOMMEL 3 , T. XXII, p. $663, x=0(1) 60(2) 100$

6. $4 \mathrm{D}, \frac{1}{2} V_{3}(x, 0), \frac{1}{2} V_{3 / 2}(x, 0), 4 M_{2}^{2}$, IGNATOWSKY, $(x / \pi)^{3}=0(.1) 8.5$

7. $4 \mathrm{D}, \frac{1}{2} V_{3}(x, 0), \frac{1}{2} V_{3 / 2}(x, 0), 2 M_{2}^{2}$, FRESNEL, $(x / \pi)=0(.1) 5.5$

8. $4 \mathrm{D}$, (a) $\frac{1}{4}\left\{[1+C(u)+S(u)]^{2}+[C(u)-S(u)]^{2}\right\}=4+V_{3}^{2}(2 z, 0)+V_{3 / 2}^{2}(2 z, 0)$ $+2 \sin \left(z-\frac{1}{3} \pi\right) V_{3}(2 z, 0)+4 \cos \left(z-\frac{1}{4} \pi\right) V_{3 / 2}(2 z, 0), u=(2 z / \pi)^{\frac{3}{3}} ;$ and (b) $\frac{2}{4}\left\{[1-C(u)-S(u)]^{2}+[C(u)-S(u)]^{2}\right\}=V_{1 / 2}^{2}(2 z, 0)+V_{3 / 2}^{2}(2 z, 0)$, Mayall (a) $u=.01(.01) .1(.05) 1$; (b) $u=.05(.05) 1$

9. $6 \mathrm{D}, a=\frac{1}{2} \cos \left(\frac{1}{2} x+\frac{1}{2} \pi\right)-\frac{1}{2} U_{3 / 2}(x, 0), b=\frac{1}{2} \sin \left(\frac{1}{2} x+\frac{1}{2} \pi\right)+\frac{1}{2} U_{3}(x, 0)$, $M_{1}^{2}=a^{2}+b^{2}=\left(\right.$ Liverpool Index) $\frac{1}{2}\left[\frac{1}{2}+C(x / 2 \pi)^{3}\right]^{2}+\frac{1}{2}\left[\frac{1}{2}+S(x / 2 \pi)^{3}\right]^{2}$, LOMMEL 3 , T. XXI, p. 661f, $x=0(1) 60(2) 100$; also, to $6 \mathrm{D}, 16$ maxima and minima of $M_{1}{ }^{2}$

10. 6D, $(\pi / 2 y)^{3} U_{3}(y, 0),(\pi / 2 y)^{3} U_{3 / 2}(y, 0), M^{2}=(\pi / 2 y)\left[U_{1}^{2}+U_{z / 2}^{2}\right]$, LOMMEL $3, \mathrm{~T}$. V, p. 652, $y=0(1) 60$; T. Va, 9 max. and min. of $M^{2}$

11. $5 S, M_{0}=(\pi x / 2)^{3} U_{1}(x, 0), x M_{0}, M_{0}^{\prime}, x^{3} M_{0}^{\prime}, x^{2} M_{0}^{\prime}$, LAMB $5, x=0(1) 60$. Also graphs of $M_{0}\left(t^{2}\right), 0<t<8$ (fig. 1); $\frac{1}{x} M_{0}\left(\frac{1}{x}\right), 0<x<.5$ (fig. 2A); $t M_{0}^{\prime}\left(t^{2}\right), 0<t<8$ (fig. 3); $\frac{1}{x^{2}} M_{0}^{\prime}\left(\frac{1}{x}\right), 0<x<.5$ (fig. $4 \mathrm{~A}$ )

12. 5D; 6D; 5D, $N_{0}=(\pi / 2 x)^{3} U_{3 / 2}(x, 0) ; N_{0} / x ; N_{0} / x^{3}$, TERAZAWA, $x=0(1) 60$

13. $4 \mathrm{D}, \frac{1}{2}\left[1-C(m-q)^{\frac{3}{3}}-C(m+q)^{3}\right]^{2}+\frac{1}{2}\left[1-S(m-q)^{\frac{1}{3}}-S(m+q)^{3}\right]^{2}$, IGNATOWSKY, $m=.5, q=0(.3) 1.8,2(.1) 8 ; m=1.5, q=0(.1) 7 ; m=3, q=0(.1) 5.5 ; m=q=0(.1) 4.2$

\section{A2. Lommel functions of two variables}

1. $7 \mathrm{D},(2 / y) U_{n} \equiv(2 / y) U_{n}(y, z)$, LoMmeL 2 , p. $269, n=1(1) 6$

2. 6D, $(2 / y) U_{1},(2 / y) U_{2}, M^{2}=(2 / y)^{2}\left[U_{1}^{2}+U_{2}^{2}\right]$, LOMMEL 2, T. III-XII, p. 317f, $z=0(1) 12$, and plates of figures:
(a) T. III, $y=\pi, 3$ minima, 3 maxima, and fig. 2, $100 M^{2}$
(b) T. IV, $y=2 \pi, 3$ min., 3 max., and fig. 3, $100 \mathrm{M}^{2}$
(c) T. V, $y=3 \pi, 3$ min., 3 max., and fig. $4,100 M^{2}$
(d) T. VI, $y=4 \pi, 2$ min., 3 max., and fig. $5,100 M^{2}$
(e) T. VII, $y=5 \pi, 3$ min., 3 max., and fig. $6,100 \mathrm{M}^{2}$
(f) T. VIII, $y=6 \pi, 2$ min., 2 max., and fig. 7, $100 M^{2}$
(g) T. IX, $y=7 \pi, 3$ min., 2 max., and fig. $8,1000 \mathrm{M}^{2}$
(h) T. X, $y=8 \pi, 2$ min., 2 max., and fig. 9, $1000 \mathrm{M}^{2}$
(i) T. XI, $y=9 \pi, 3$ min., 2 max., and fig. 10, $1000 M^{2}$
(j) T. XII, $y=10 \pi, 2$ min., 2 max., and fig. $11,1000 M^{2}$

These minima and maxima correspond to zeros of $U_{2}(x)$ [first $3, y=0$ of $J_{1}(x)$ ], see no. 3

3. $6 \mathrm{D}, z$ and $\tan ^{-1}(\partial y / \partial z)$ to $1^{\prime \prime}, 3$ zeros of $U_{2}(x)$ [the first $3, y=0$, of $J_{2}(x)$ ], Lommel 2, T. XIII, p. 322, and fig. 12, $y=0(\pi) 10 \pi, 12(1) 14,20.5,26.2$

4. 6D, $(2 / z) U_{1}(z, z),(2 / z) U_{2}(z, z), M^{2}$, LoMmeL 2 , T. XV, p. $323, z=0(.5) 12$ and fig. 13 , $y=2,100 M^{2}$

5. 6D, $-(2 / y) V_{1}(y, z),(2 / y) V_{0}(y, z), M_{1}^{2}=(2 / y)^{2}\left(V_{0}^{2}+V_{1}^{2}\right)$, LOMMEL $2, z=0(1) 12$, T. XVI-XXIII, p. 323-327 and plates of figures:

(a) T. XVI, $y=\pi, 6$ min., 5 max., and fig. $14,10 M_{1}^{2}$

(b) T. XVII, $y=2 \pi, 4$ min., 4 max., and fig. $15,100 M_{1}^{2}$

(c) T. XVIII, $y=3 \pi, 4$ min., 3 max., and fig. 16, $100 M_{1}^{2}$

(d) T. XIX, $y=4 \pi, 4$ min., 3 max., and fig. $17,100 M_{1}^{2}$

(e) T. XX, $y=5 \pi, 4$ min., 3 max., and fig. $18,1000 M_{1}^{2}$

(f) T. XXI, $y=6 \pi, 4$ min., 3 max., and fig. $19,1000 M_{1}^{2}$

(g) T. XXII, $y=7 \pi, 4$ min., 3 max.

(h) T. XXIII, $y=8 \pi, 4$ min., 3 max.

These maxima and minima correspond to zeros of $J_{1}(y)$ and $V_{0}(y, z)$; see no. 6

6. $6 \mathrm{D}, z$, and $\tan ^{-1}(\partial y / \partial z)$ to $1^{\prime \prime}$, zeros of $V_{0}(y, z)$, LoMmeL 2, T. XXIV, p. $327, y=\pi(\pi) 8 \pi$ and fig. $20\left(J_{1}=0, V_{0}=0\right)$ 
7. $6 \mathrm{D},(2 / z) V_{1}(z, z),(2 / z) V_{0}(z, z), M_{1}^{2}$, LoMmeL $2, \mathrm{~T}$. XXV, p. $328, z=0(.5) 12$, and fig. $21,100 M_{1}^{2}$

8. $6 \mathrm{D},(\pi / 2 y)^{3} U_{\mathrm{j}}(y, z),(\pi / 2 y)^{3} U_{3 / 2}(y, z), M^{2}$, LOMmeL 3 , T. VI-XV, p. $653 \mathrm{f}, y=3(3) 30$, $z=0(1) 12$; $\max$. and $\min$. for $z=\pi(\pi) 4 \pi$. Figs. 2-11, $100 M^{2}$

9. 6D, $(\pi / 2 y)^{3} V_{1}(y, z),(\pi / 2 y)^{3} V_{8 / 2}(y, z), M_{1}^{2}$, LOMMEL 3, T. XVII-XX, p. 659f, $y=3(3) 12$, $z=0(1) 12$; and $\max$. and $\min$. for $z=\pi(\pi) 4 \pi$. Figs. $13-15, y=3(3) 12,10 M_{1}^{2}$

\section{B. Series}

1. 5-6D, $\frac{1}{2} \pi\left[J_{0}(x)-\frac{1}{2} n J_{1}(x) / x+(n-4) 3 n J_{2}(x) / 32 x^{2}-\cdots\right]$, JAHNKE, values for $n=$ $3,5, x=10 / \pi$

2. 5D, $C(\theta)=\sum_{\theta=1}^{\infty}\left\{2 /\left[x_{s} J_{1}\left(x_{s}\right)\right]\right\} e^{-x_{s}^{2} \theta}, \log C(\theta), \theta=k t / r^{2}$, OLsON \& SCHULtz, $\theta=.001(.001) .4, x_{1}$ zeros of $J_{0}(x)$

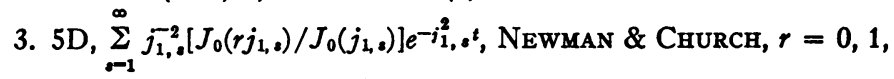
$t=0(.005) .01(.01) \cdot 1(.02) .2(.05) \cdot 5,1$

4. $4 \mathrm{D}, \sum_{s=1}^{\infty} J_{0}\left(r x_{s}\right) e^{-z x_{s}} /\left[x_{s} n J_{1}\left(x_{s}\right)\right]$, BeRTRAM, $r=0(.1) .9 ; n=1, z=0(.05) 1.75 ; n=2$, $z=0(.05) 1.5 ; n=3, z=0(.05) 1 ; n=4, z=0(.05) 1$

5. 4D, $\sum_{m=1}^{\infty} J_{0}(m x), \sum_{m=1}^{\infty} Y_{0}(m x)$, WESSEL, $x=.3(.3) 13.5$; also graphs of the functions

6. $4 \mathrm{~S}, \stackrel{m-1}{F}=\left(2 \pi / x^{2}\right)\left[H_{0}^{-2} \cdot H_{1}^{-2}+2 H_{1}^{-2} \cdot H_{2}^{-2} x^{-8}\left\{x^{2}-3(1-m)\right\}^{2}\right.$ $\left.+\sum_{n=2}^{\infty}\left\{(n+1) /\left(H_{n}^{2} H_{n+1}^{2} x^{4 n+4}\right)\right\}\left\{x^{2}-n /(n+2)\right\}^{2}\right]$ where

$H_{n}{ }^{2}=\left(\pi / 2 x^{2 n+1}\right)\left[n^{2}\left\{J_{n+1}^{2}(x)+J_{-n-1}^{2}(x)\right\}+2 n x\left\{J_{-n-1}(x) J_{-n-3 / 2}(x)-J_{n+3}(x) J_{n+3 / 2}(x)\right\}\right.$ $\left.+x^{2}\left\{J_{n+3 / 2}^{2}(x)+J_{-n-3 / 2}^{2}(x)\right\}\right], n \neq 1, H_{1}^{2}=\left(\pi / 2 x^{8}\right)\left[(1-m)^{2}\left\{J_{3 / 2}^{2}(x)+J_{-3 / 2}^{2}(x)\right\}\right.$ $\left.+2 x(1-m)\left\{J_{-8 / 2}(x) J_{-5 / 2}(x)-J_{8 / 2}(x) J_{5 / 2}(x)\right\}+x^{2}\left\{J_{5 / 2}^{2}(x)+J_{-5 / 2}^{2}(x)\right\}\right]$,

Fox, Table of $F$ for $x=1(1) 11,15,20, \pi, m=0(.1) .2, .5,1$; in another table $Y=z F / \pi x^{2}$ is given for the same ranges of $x$ and $m$. The $5 Y^{\prime}$ 's are plotted for $2<x<15$

7. $3 \mathrm{D}, r^{2}-1-8 \sum_{s=1}^{\infty}\left[J_{0}\left(r x_{s}\right) /\left\{x_{e}{ }^{3} J_{1}\left(x_{s}\right)\right\}\right] e^{-x_{0}^{2} t}$, SZYMANSKI, $r=0(.1) 1$, $t=.05(.05) \cdot 2(.1) \cdot 5(.25) 1$

8. 3D, $\left(\frac{1}{3} \pi n\right)^{-i} \sum_{n=0}^{\infty}(2 n+1) i^{n} P_{n}(\cos \theta) / N$, Ballantine, $N=(-1)^{n}\left[(n+1) J_{-n-1}(x)+x J_{1-n}(x)\right]-i\left[(n+1) J_{n+\}}(x)-x J_{n-1}(x)\right]$, $x=.1(.1) .3(.2) .7, .85,1(1) 4,6,10 ; \theta=0$

9. $3-4 \mathrm{~S}, \sum_{n=1}^{\infty}(2 n+1) j_{0, n}(x) /[4 n(n+1)], \sum_{n=1}^{\infty} \frac{1}{2}(2 n+1) j_{0, n}^{2}(x)$, WANNIER, $x=0(5) 10(10) 80$

\section{Graphs}

1. $z=\sum_{n=-\infty}^{\infty} x^{2} J_{n}^{2}(i y) /\left(x^{2}+n^{2}\right)$, ERDELYYI, $0<y<3, x=0(.4) 2$

2. $A_{2 n}(x)=(-1)^{n}(4 n+1)\left[x^{-1}-\left(\frac{1}{2} \pi\right)^{+}\left(1 / 2^{n}\right)\right] \sum_{m=0}^{n} C_{m, n} x^{-m-j} J_{m-1}(x)$, BackHAUs 2, $C_{m, n}=(-1)^{n-m}(2 m+2 n-1) ! ! /[m !(n-m) !]$, fig. $3, n=0(1) 6,0<x<15$; fig. 4, graph of $A_{2 n}(x)$ against $0<n<19, x=5,8,11,14,17$

3. $1-\sum_{n=1}^{\infty}\left(2 / x_{n}\right)\left[J_{0}\left(r x_{n}\right) / J_{1}\left(x_{n}\right)\right] e^{-x_{n}^{2} t}$, W. MÜLLER, p. $189,0<r<1, t=.01,025$, $.05, .1, .2, .3, .5$

4. $2\left(1-x^{2}\right)+\sum_{n=1}^{\infty}\left(\frac{U}{u}-\frac{8}{x_{n}^{2}}\right)\left(\frac{2}{x_{n}}\right) \frac{J_{0}\left(x x_{n}\right)}{J_{1}\left(x_{n}\right)} e^{-x_{n}^{2} t}$, W. MÜLLER, p. 201, $|x|<1$, value of $U / u$ not specified, $t=0, .005, .0125, .025, .05, .1, .25, .5, \infty$ 
5. $\sum_{n=1}^{\infty}\left(2 b / x_{n}\right)\left[1 /\left(x_{n}^{4}+b^{2}\right)\right]\left[J_{0}\left(r x_{n}\right) / J_{1}\left(x_{n}\right)\right] e^{-x_{n}^{2} t}$

$+2 \sin (b t) \sum_{n=1}^{\infty}\left[x_{n} /\left(x_{n}^{4}+b^{2}\right)\right]\left[J_{0}\left(r x_{n}\right) / J_{1}\left(x_{n}\right)\right]$

$-2 \cos (b t) \sum_{n=1}^{\infty} \frac{b}{x_{n}}\left[1 /\left(x_{n}^{4}+x_{n} b^{2}\right)\right]\left[J_{0}\left(r x_{n}\right) / J_{1}\left(x_{n}\right)\right]$, W. MÜLLER, p. 203, r=.2(.2).8,

$0<b t<5 \pi / 2$

6. $r t-(r / 8)\left(1-r^{2}\right)-\sum_{n=1}^{\infty}\left(2 / j_{1, n}^{2}\right)\left[J_{1}\left(r j_{1, n}\right) / J_{0}\left(j_{1, n}\right)\right] e^{-j_{1, n}^{2}}$, W. MÜLLER, p. 195, $0<r<1$, $0<t<1.4$

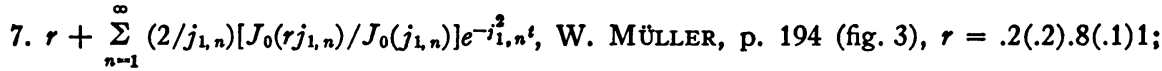
$0<t<.25$

8. $1-e^{-a^{2} t}+2 a^{2} \sum_{n=1}^{\infty} \frac{e^{-j_{1, n^{t}}^{2}-e^{-a^{2} t}}}{a^{2}-j_{1, n}^{2}}$, W. MÜLLER, p. 194 (fig. 4), $a^{2}=10,20,50,100, \infty$; $0<t<.14$

9. $r\left(1-e^{-a^{2} t}\right)+2 \sum_{n=1}^{\infty} \frac{a^{2}}{a^{2}-j_{1, n}^{2}}\left[J_{0}\left(r j_{1, n}\right) /\left\{j_{1, n} J_{0}\left(j_{1, n}\right)\right\}\right] e^{-\left(a^{2}+j_{1, n}^{2}\right) t}$, W. MÜLLER, p. 194 (fig. 2), $a=10, r=.2(.2) .8(.1) 1 ; t=0(.05) \cdot 25$

10. $2 \cos (b t) \sum_{n=1}^{\infty}\left[j_{1, n} /\left(j_{1, n}^{4}+b^{2}\right)\right]\left[J_{1}\left(r j_{1, n}\right) / J_{0}\left(j_{1, n}\right)\right]$

$-2 \sin (b t) \sum_{n=1}^{\infty}\left[j_{1, n}^{2} /\left(j_{1, n}^{4}+b^{2}\right)\right]\left[J_{1}\left(r j_{1, n}\right) / J_{0}\left(j_{1, n}\right)\right]-2 b \sum_{n=1}^{\infty}\left[j_{1, n} /\left(j_{1, n}^{4}+b^{2}\right)\right]$

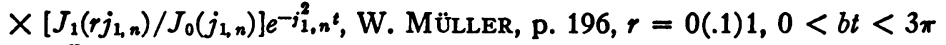
11. $4 x \sum_{k=18.6}^{\infty} \cos (k \pi t) K_{1}(k \pi x) /\left[k x K_{0}(k \pi x)\right]$, OLlendoRfF \& SEeliger, $x=.01, .1,1, \infty$;
$0<t<1$

See also I $B_{3} 9,16,17 ; B_{6} 6,7 ; \operatorname{XII~} A_{1} 11 ; A_{2} 2-9 ; B$

\section{Polynomial Approximations and AsYMPTOTIC EXPANSIONS}

\section{A. Introduction}

When Abraham de Moivre and James Stirling obtained their famous estimate of $n$ ! for large values of $n$, the rough estimate

$$
(2 \pi n)^{\frac{3}{3}} n^{n} e^{-n}
$$

was improved by a factor which was obtained most conveniently by means of an expression for the logarithm of $n$ ! in which the additional terms formed part of a divergent infinite series in $1 / n$. This result was generalized by EULER into the sum formula

$$
\begin{aligned}
f(1)+f(2)+\cdots+ & f(n-1) \\
& =\int f(x) d x-\frac{1}{2} f(n)+B_{1} f^{\prime}(n) / 2 !-B_{2} f^{\prime \prime \prime}(n) / 4 !+\cdots
\end{aligned}
$$

which had already been found by MACLAURIN, the coefficients $B_{m}$ being the well-known numbers of JACQUES BERNOULLI.

When $f(x)=\log x$ and when $f(x)=x^{-p}$, the series involving these numbers is a power series in $1 / x$ and, according to the description of Euler's 
work given by BROMwICH, ${ }^{1}$ the rule for the truncation of the series is to stop at a stage when the error is less than the next term of the series. Such a series was called a semiconvergent series. The term asymptotic expansion was applied to such a series when HENRI PoINCARé ${ }^{2}$ gave the following rule: The polynomial

$$
P(x, m)=a_{0}+a_{1} x^{-1}+a_{2} x^{-2}+\cdots+a_{m} x^{-m}
$$

may be used to estimate $F(x)$ for large values of $x$ when the difference $F(x)-P(x, m)$ has a modulus less that $K_{m} /|x|^{m+1}$, where $K_{m}$ depends on $m$ but not on $x$. This rule gives the limit

$$
\lim _{x \rightarrow \infty}\left|x^{m}\{F(x)-P(x, m)\}\right|=0
$$

and as the infinite series $P(x, \infty)$ may be divergent, use may be made of the notation

$$
F(x) \approx a_{0}+a_{1} x^{-1}+a_{2} x^{-2}+\cdots .
$$

\section{B. Asymptotic series for $J_{n}(x)$ and $Y_{n}(x)$}

When $n$ is fixed and $x$ is a large positive number much exceeding $n$, the semiconvergent series of $\mathrm{Jacobi}^{3}$ gave a generalization to all integral values of $n$ of Poisson's estimates ${ }^{4}$ of $J_{n}(x)$ for $n=0,1$ and of Cauchy's estimate ${ }^{5}$ for $J_{2}(x)$. If $X_{n}=x-\frac{1}{2}\left(n+\frac{1}{2}\right) \pi, 2 i x z=1$ the series in question is

where

$$
J_{n}(x) \approx\left(\frac{1}{2} \pi x\right)^{-\frac{1}{2}}\left[u_{n}(x) \cos X_{n}-v_{n}(x) \sin X_{n}\right],
$$

$$
u_{n}(x)+i v_{n}(x)=r_{n}(x) \exp \left[i a_{n}(x)\right]={ }_{2} F_{0}\left(\frac{1}{2}+n, \frac{1}{2}-n ; z\right)
$$

and use is made of the notation of BARNES ${ }^{6}$ for the generalized hypergeometric function

$$
\begin{gathered}
{ }_{p} F_{q}\left(a_{1}, a_{2}, \cdots a_{p} ; c_{1}, c_{2}, \cdots c_{q} ; z\right)=\sum_{n=0}^{\infty} \frac{z^{n}}{n !} \frac{\left(a_{1}\right)_{n}\left(a_{2}\right)_{n} \cdots\left(a_{p}\right)_{n}}{\left(c_{1}\right)_{n}\left(c_{2}\right)_{n} \cdots\left(c_{q}\right)_{n}}, \\
(a)_{n}=\frac{\Gamma(a+n)}{\Gamma(a)} .
\end{gathered}
$$

When $n+\frac{1}{2}$ is an integer the series terminates and ${ }_{2} F_{0}(p, 1-p ; z)$ is the polynomial used by IVORY ${ }^{7}$ in his work on refraction. The existence of this polynomial was known from the cases in which the solution of Riccati's equation can be expressed in finite terms. When $n$ is an integer the series is infinite and much work has been done to find estimates of error when the series is truncated. The researches of Stieltjes and Callandreau are summarized by WATSON ${ }^{8}$ and elucidated. It is found among other things that after a certain stage the remainder is less in absolute value than the term at which the series is stopped and has a sign opposite to this term. This is true for both $u_{n}(x)$ and $v_{n}(x)$. The rank of the smallest term in each series is of order $x$. Further work on the remainders has been done by Koshliakov ${ }^{9}$ and Strogonoff. ${ }^{10}$ AIREy ${ }^{11}$ has found convergence factors of type $f=\frac{1}{2}+c$ to be associated with the smallest term of a semiconvergent series by using a special rearrangement of the terms of a repeated series and the accuracy of the estimate given by a truncated series is thus 
improved. He has also shown that the semiconvergent series for $J_{0}(x)$, $J_{1}(x)$ can be used for accurate calculations up to $12 \mathrm{D}$ when $x \geq 8$.

GREGORY and LODGE have studied the functions $r_{n}(x), a_{n}(x)$, the work being continued by LODGE ${ }^{12}$ after Gregory's death. It was shown that

$$
\left[r_{n}(x)\right]^{2}={ }_{3} F_{0}\left(\frac{1}{2}, \frac{1}{2}+n, \frac{1}{2}-n ;-x^{-2}\right) .
$$

This formal identity which was given also by NIELSEN ${ }^{13}$ is useful for the computation of $r_{n}(x)$ and is a particular case of the more general formula

$$
{ }_{2} F_{0}(a, b ; x){ }_{2} F_{0}(a, b ;-x)={ }_{4} F_{1}\left(a, b, \frac{1}{2} a+\frac{1}{2} b, \frac{1}{2} a+\frac{1}{2} b+\frac{1}{2} ; a+b ; 4 x^{2}\right) .
$$

A semiconvergent expansion

$$
\begin{aligned}
a_{n}(x) \approx k u+k(k-3) u^{3} / 6+ & k\left(k^{2}-14 k+15\right) u^{5} / 10 \\
& +k\left(5 k^{3}-190 k^{2}+807 k-630\right) u^{5} / 56+\cdots
\end{aligned}
$$

in which $u=1 / x, 8 k=4 n^{2}-1$, was also found to be useful for computation and it was shown that

$$
\sec \left[a_{n+1}(x)-a_{n}(x)\right]=r_{n}(x) r_{n+1}(x),
$$

a result equivalent to the identity

$$
u_{n}(x) u_{n+1}(x)+v_{n}(x) v_{n+1}(x)=1 .
$$

This was first found by induction and then proved directly by M. J. M. HILL.

The tables of LoDGE are listed in IV D 4, 5. They refer to both integral and half-integral values of $n$.

The BAASMTC later tabulated the functions $A_{0}, A_{1}, B_{0}, B_{1}$ which are defined by means of the equations

$$
\begin{aligned}
& J_{0}(x)+i Y_{0}(x)=\left[B_{0}(x)-i A_{0}(x)\right] e^{i x}, \\
& J_{1}(x)+i Y_{1}(x)=-\left[A_{1}(x)+i B_{1}(x)\right] e^{i x},
\end{aligned}
$$

where the notation $Y_{n}(x)$ is used for the Bessel function of the second kind whose asymptotic form is

$$
Y_{n}(x) \approx\left(\frac{1}{2} \pi x\right)^{-\frac{2}{2}}\left[u_{n}(x) \sin X_{n}+v_{n}(x) \cos X_{n}\right] .
$$

These tables are described in II $\mathbf{A}_{3}$.

\section{Asymptotic series for $\boldsymbol{I}_{n}(x)$ and $\boldsymbol{K}_{n}(x)$}

The asymptotic expansions were soon extended to Bessel functions of purely imaginary argument and when $x$ is positive the functions $I_{n}(x)$ and $K_{n}(x)$ may, for large values of $x$ much exceeding $n$, be computed from the expansions

where

$$
\begin{aligned}
I_{n}(x) & \approx(2 \pi x)^{-\frac{1}{2}}\left[e^{x} w_{n}(x)+e^{-x} w_{n}(-x)\right], \\
K_{n}(x) & \approx(2 x / \pi)^{-\frac{1}{2}} e^{-x} w_{n}(-x),
\end{aligned}
$$

and so

$$
w_{n}(i y)=u_{n}(y)+i v_{n}(y)={ }_{2} F_{0}\left(\frac{1}{2}+n, \frac{1}{2}-n ; 1 /[2 i y]\right),
$$

$$
w_{n}(x)={ }_{2} F_{0}\left(\frac{1}{2}+n, \frac{1}{2}-n ; \frac{1}{2} x^{-1}\right) .
$$


The effect of truncating these series was studied by Stieltjes and convergence factors of type $f=\frac{1}{2}+c$ for the smallest term have been found by Burnett after Airey had given the factors for the series $w_{0}(x)$ and $w_{1}(x)$. The results are as follows:

If $m-1<x<m+1$ where $m$ is an integer and $x=m+t$, then, according to BURNETT ${ }^{14}$

$$
f=\frac{1}{2}+(1+2 t) / 8 x+\left(2 n^{2}-16+4 t+8 t^{2}\right) /(8 x)^{2}+\cdots .
$$

KING ${ }^{15}$ gives the estimate

$$
\int_{0}^{x} e^{u} K_{0}(u) d u+C \approx(2 \pi x)^{+1}\left[1+1 /(8 x)-\frac{1^{2} \cdot 3}{2 !(8 x)^{2}}+\frac{1^{2} \cdot 3^{2} \cdot 5}{3 !(8 x)^{3}}-\cdots\right]
$$

and remarks that the constant $C$ is probably unity, judging from some numerical calculations. It may be noticed, however, that

$$
\int_{0}^{\infty}\left[\pi e^{-u} I_{0}(u)-e^{u} K_{0}(u)\right] d u=1,
$$

and so the value 1 of the constant may be inferred from the asymptotic form of

$$
\int_{0}^{x} e^{-u} I_{0}(u) d u
$$

The constant in the asymptotic expansion for $\int_{0}^{x} e^{-u} I_{0}(u) d u$ may be found as follows:

$$
\int_{0}^{\infty} e^{-u}\left[I_{0}(u)-I_{n}(u)\right] d u=\lim _{0 \rightarrow 0} \frac{1-e^{-n v}}{\sinh v}=n, \quad(n>-1)
$$

and so

But

$$
\int_{0}^{\infty} e^{-u}\left[I_{3}(u)+I_{-3}(u)-2 I_{0}(u)\right] d u=0 .
$$

$$
\pi \int_{0}^{x} e^{-u}\left[I_{3}(u)+I_{-3}(u)\right] d u=\int_{0}^{x}(2 \pi / u)^{\frac{3}{3}} d u=2(2 \pi x)^{3}
$$

and contains no constant term, consequently there is no constant term in the asymptotic expansion of

$$
\int_{0}^{x} e^{-u} I_{0}(u) d u
$$

The constant term in the asymptotic expansion of $\int_{0}^{x} e^{-u} I_{n}(u) d u$ seems to be $-n$ when $n>-1$.

\section{Approximations by means of series of various types}

The subject of asymptotic expansions is part of the more extensive subject of the approximation to functions by means of series of special functions. Polynomials in $x$ or $(1 / x)$ are the simplest special functions which 
can be used to represent the terms or partial sums of such series but rational functions occur, for instance, when a continued fraction provides the successive convergents, and other special functions have advantages in certain cases.

In the case of polynomials there is the theorem of WEIERSTRAss ${ }^{16}$ that any continuous function can be uniformly approximated in a given real interval (or region of the complex plane) by means of a polynomial. The coefficients of this polynomial are generally different for different degrees of the polynomial and are different also from the coefficients of the different powers of the variable in the Maclaurin expansion. Series of Legendre polynomials may with a few terms give a much better approximation to a function in the interval $-1<x<1$ than the Taylor series in ascending powers of $x$ and similarly for other series of orthogonal functions.

In the complex domain the region in which a series gives a good approximation to a function is generally limited and sometimes hard to specify. The importance of this in the case of asymptotic series was recognized by STOKES ${ }^{17}$ who pointed out that as the argument of $z$ varies in an asymptotic series representing a solution of a differential equation the constant multipliers in the representation of this solution by means of standard solutions associated with $z=0$ may not always be the same. This is generally called the phenomenon of Stokes and on this account it is usual to specify the range of argument for which the asymptotic expansion of a given function is valid for the complex plane.

In Hankel's work ${ }^{18}$ combinations of Bessel functions are formed so that in each case the problem of forming an estimate for large values of the variable reduces to that of approximating a single function of $z$ by means of a polynomial in $1 / z$. When $n$ is an integer the Hankel functions are defined by means of the equations

$$
H_{n}{ }^{(1)}(z)=J_{n}(z)+i Y_{n}(z), \quad H_{n}{ }^{(2)}(z)=J_{n}(z)-i Y_{n}(z) .
$$

For positive values of $x$

$$
H_{n}^{(1)}(x) \approx\left(\frac{1}{2} \pi x\right)^{-1} e^{i x_{n}}\left[u_{n}(x)+i v_{n}(x)\right] .
$$

As Bessel functions of half integral order occurred in acoustics and as the work of Stokes on the integral of Airy indicated that this function was related to the Bessel functions of orders $\pm 1 / 3$, the definitions of Hankel's functions were extended to non-integral and even complex values of $n$ by means of the equations

$$
\begin{aligned}
& H_{n}{ }^{(1)}(z)=i \csc (n \pi)\left[e^{-i n \pi} J_{n}(z)-J_{-n}(z)\right], \\
& H_{n}{ }^{(2)}(z)=-i \csc (n \pi)\left[e^{i n \pi} J_{n}(z)-J_{-n}(z)\right],
\end{aligned}
$$

and asymptotic expansions were developed which are particularly useful for the selection of functions representing diverging waves. These expansions were given also for complex values of the variable and were found to be useful in problems of electromagnetic theory when waves are propagated in an absorbing medium. It is then necessary to specify the limitations to which the argument of $z$ is subjected in order that a particular type of 
asymptotic representation may hold. If $\delta$ denotes any positive acute angle and

$$
\begin{gathered}
-\pi+2 \delta \leq \arg z \leq 2 \pi-2 \delta, \quad R\left(v+\frac{1}{2}\right)>0 \\
H_{v}^{(1)}(z)=(2 / \pi z)^{\frac{3}{2}} e^{i z} e^{-\frac{j}{i} i\left(v+\frac{1}{2}\right) \pi}\left[\sum_{m=0}^{p-1} \frac{\left(\frac{1}{2}-v\right)_{m}\left(\frac{1}{2}+v\right)_{m}}{m !(2 i z)^{m}}\right]+\mathrm{O}\left(z^{-p}\right) .
\end{gathered}
$$

LANCzos ${ }^{19}$ has recently extended to the complex domain some of the ideas relating to approximations by means of polynomials or series of orthogonal functions. In particular, the usual asymptotic expansions are of ten replaced by expansions of similar form but with modified coefficients which decrease the error in the approximation. When the function satisfies a linear differential equation with rational coefficients, the factors of the terms of the modified series can be found by means of recurrence relations which, however, make them depend on the degree of the approximating polynomial. This is true in any type of approximation by polynomials which differs from the expansion in a Taylor series. The methods of Lanczos have been used with some success by the computers of the Mathematical Tables Project of New York. Approximations by series of various types are obtained when use is made of methods of successive approximations for differential or integral equations. The case in which the equation contains a parameter was treated by LIOUVILLE ${ }^{20}$ in 1837 and his lead has been followed by KNESER, ${ }^{21}$ Horn, ${ }^{22}$ Birkhoff, ${ }^{23}$ Hobson ${ }^{24}$ and others. Convergent series of approximation have been obtained by HoHEISEL ${ }^{25}$ and by IKEDA. ${ }^{26}$ The latter deals with Bessel's equation by means of a transformation and an integral equation. In the method of Horn the aim is to solve $y^{\prime \prime}+k^{2} Q y=0$ by means of a series

$$
y=e^{i k w}\left[A_{0}+A_{1} k^{-1}+A_{2} k^{-2}+\cdots\right],
$$

and it is found first of all that $w=\int_{a}^{x} Q_{0}{ }^{-\frac{1}{3}} d x$, where $Q_{0}$ is the first term in the expansion

$$
Q=Q_{0}+Q_{1} k^{-1}+Q_{2} k^{-2}+\cdots,|k|>R .
$$

This method has been much used by JEFFrEys ${ }^{27}$ and others and is known in quantum theory as the B-K-W-method after BrILLOUIN, ${ }^{28}$ Kramers ${ }^{29}$ and WENTZEL. ${ }^{30}$ Jeffreys and GolDSTEIN ${ }^{31}$ have made special studies of the case in which $Q_{0}$ becomes zero at some point which has to be considered in forming $w$ and have elucidated the different cases by examples from the theory of Mathieu functions and Bessel functions. When $n$ and $z$ are both large a comparison between the differential equation satisfied by $J_{n}(z)$ after a suitable transformation and the differential equation for the Airy functions $A i(x), B i(x)$ proves useful. Results may then be expressed in terms of these functions and the tables of $\mathrm{VB}_{2} 3,4$ are then useful. In some cases results are expressed in terms of Bessel functions of orders $\pm 1 / 3$ and WATSON ${ }^{32}$ made the tables in $\mathrm{VA}_{1} 3,4,10 ; \mathbf{B}_{1} 3 ; \mathbf{B}_{2} 4$. He found that the series $u_{n}(x), v_{n}(x)$ can be used with the desired accuracy for $x>8$ and that $u_{n}(x)>0, v_{n}(x)>0$, $u_{n}(x) v_{n}^{\prime}(x)>u_{n}^{\prime}(x) v_{n}(x)$. 
E. Asymptotic expansions of Bessel functions of nearly equal order and argument

Estimates of $J_{n+m}(n e)(0<e<1)$ were needed for the discussion of the convergence of the series of planetary theory, and the early work of CARLINI ${ }^{33}$ was completed by JACOBI ${ }^{34}$ and others. Similar estimates were needed for physical problems of wave propagation and much progress was made when DEBYE, ${ }^{35}$ WATSON ${ }^{36}$ and others used the method of the saddle point for the evaluation of integrals in the complex plane. When use is made of the Airy functions, the results of Watson may be written in the following form:

If $p$ is large and $x=q^{3} /\left(3 p^{2}\right)$ is arbitrary then

where

$$
\begin{aligned}
& J_{p}\left[\left(p^{2}-q^{2}\right)^{\frac{1}{2}}\right] \approx(2 / p)^{1 / 3} e^{q+x-p} \tanh ^{-1}(q / p) A i[z], \\
& J_{p}\left[\left(p^{2}+q^{2}\right)^{\frac{1}{1}}\right] \approx(2 / p)^{1 / 3}[A i(-z) \cos w+B i(-z) \sin w], \\
& Y_{p}\left[\left(p^{2}+q^{2}\right)^{\frac{1}{3}}\right] \approx(2 / p)^{1 / 3}[A i(-z) \sin w-B i(-z) \cos w],
\end{aligned}
$$

$$
z=(3 x / 2)^{2 / 3} \quad \text { and } \quad w=q-x-p \tan ^{-1}(q / p) .
$$

For a more complete account reference may be made to the book of Watson and to the more recent work of VAN VEeN, ${ }^{37}$ PollaczeK, ${ }^{38}$ LANGer, ${ }^{39}$ JAHNKE \& EMDE, ${ }^{40}$ EMdE, ${ }^{41}$ EMdE \& RÜHLE. ${ }^{42}$ Langer uses methods depending on the transformation of differential equations and his results apply also to the case in which $n$ is complex. This case is needed in some wave problems and AIREy ${ }^{43}$ has given an explicit form for the asymptotic expansion of the function $J_{i}(x)$ of imaginary order which occurs in the work of Boole.

In terms of the Bessel functions of orders $\pm 1 / 3$ the results are as follows:

If $0<x<\infty, 0<a<\infty, 0<b<\frac{1}{2} \pi, u=\pi / 6, \quad T=\tan b-b$, $t=a-\tanh a$,

then

$$
A=(a \operatorname{coth} a-1)^{\frac{1}{3}}, \quad B=(1-b \cot b)^{\frac{1}{3}},
$$

$$
\begin{aligned}
J_{n}(n \sec b) & \approx B\left[\cos u J_{1 / 3}(n T)-\sin u Y_{1 / 3}(n T)\right], \\
Y_{n}(n \sec b) & \approx B\left[\sin u J_{1 / 3}(n T)+\cos u Y_{1 / 3}(n T)\right], \\
H_{n}^{(1)}(n \sec b) & \approx B e^{i u / 6} H_{1 / 3}^{(1)}(n T), \\
H_{n}^{(2)}(n \sec b) & \approx B e^{-i u / 6} H_{1 / 3}^{(2)}(n T), \\
J_{n}(n \operatorname{sech} a) & \approx(A / \pi) K_{1 / 3}(n t), \\
Y_{n}(n \operatorname{sech} a) & \approx A\left[2 I_{1 / 3}(n t)+(\sqrt{3} / \pi) K_{1 / 3}(n t)\right] .
\end{aligned}
$$

For the elucidation of these results reference may be made to the papers of Nicholson, ${ }^{44}$ Rayleigh, ${ }^{45}$ Meijer, ${ }^{46}$ FoK, ${ }^{47}$ Svetlov ${ }^{48}$ and VaN VEEN. ${ }^{49}$ Rayleigh made use of the method of stationary phase, a method which mathematicians until recently viewed with some distrust. The researches of VAN DER CORPUT ${ }^{50}$ have, however, put the method on a sounder foundation and for Bessel functions the following results have been obtained by BIJL. ${ }^{51}$

If

$$
\begin{gathered}
|n-x|^{3}<x \quad \text { and } \quad s+1=3 S \\
\left|3 \pi J_{n}(x)-\sum_{s=0}^{m-1}\left(B_{s} / s !\right)(6 / x)^{s} \Gamma(S) \sin (2 S \pi)\right| \leq c w^{-m}, \\
\left|3 \pi Y_{n}(x)+\sum_{s=0}^{m-1}\left(B_{s} / s !\right)(6 / x)^{s} \Gamma(S)\{1-\cos (2 S \pi)\}\right| \leq c^{\prime} w^{-m}
\end{gathered}
$$


where $m$ is an arbitrary positive integer, $c$ and $c^{\prime}$ are constants depending on $m$ and where

$$
\begin{aligned}
& w^{15}=x^{2} \quad \text { for } \quad|n-x|^{5}<x, \\
& w^{3}=x|n-x|^{-3} \quad \text { for } \quad x^{3} \leq|n-x|^{15} \leq x^{5} \text {, } \\
& B_{0}=1, \quad B_{s}=(n-x) B_{s-1}-\sum_{r=0}^{s-1}\left[\frac{\Gamma(s)}{r ! \Gamma(s-r)}\right] x B_{s-r-1} .
\end{aligned}
$$

Also, if $n^{2} \leq x$$$
\left|K_{n}(x)-(2 x)^{-\frac{1}{3}} e^{-x} \sum_{s=0}^{m-1}\left[L_{2 s} /(2 s) !\right]\left(\frac{1}{2} s\right)^{-8} \Gamma\left(s+\frac{1}{2}\right)\right| \leq c^{\prime \prime} w^{-2 m} x^{-\frac{1}{3}},
$$

where $L_{0}=1, L_{2}=n^{2}, L_{4}=n^{4}-x, L_{6}=n^{6}-15 n^{2} x-x$, and $w^{2}=x^{\frac{1}{2}}$ or $x / n^{2}$ according as $n^{4} \leq x$ or $n^{4}>x$. When $n \geq x^{\frac{1}{2}}$ the result is

$$
\left|K_{n}(x)-(2 N)^{-\frac{1}{3}} e^{-N+n \ln M} \sum_{s=0}^{m-1}\left(P_{2 s} /(2 s) !\right)\left(\frac{1}{2} N\right)^{-s} \Gamma\left(s+\frac{1}{2}\right)\right|
$$

where

$$
\leq c w^{-m} N^{-\frac{1}{2}} e^{-N+n \ln M}
$$

$$
\begin{gathered}
N=\left(n^{2}+x^{2}\right)^{\frac{1}{2}}, N+n=M x, P_{0}=P_{1}=P_{2}=0, P_{4}=-N, \\
P_{6}=10 n^{2}-N, P_{8}=56 n^{2}+35 N^{2}-N, P_{10}=-2100 n^{2} N+246 n^{2}+210 N^{2},
\end{gathered}
$$

and where $w^{2}=n^{2} / x$ or $N / n$ according as $n^{4} \leq x^{3}$ or $n^{4}>x^{3}$.

For the computation of $I_{n}(x)$ and $K_{n}(x)$ for large values of $n$ and $x$ when $x$ exceeds $n$, the formulae of Nicholson and Erdélyi are

$$
\begin{aligned}
I_{n}(n \operatorname{cosech} a) & \approx(2 \pi n \operatorname{coth} a)^{-\frac{1}{2}} e^{n(\tanh a-a)}\left[1+\mathrm{O}\left(n^{-1}\right)\right], \\
K_{n}(n \operatorname{cosech} a) & \approx \pi(2 \pi n \operatorname{coth} a)^{-\frac{1}{2}} e^{-n(\tanh a-a)}\left[1+\mathrm{O}\left(n^{-1}\right)\right],
\end{aligned}
$$

where $a>0, n>0$.

LEHMER ${ }^{52}$ remarks that the usual formula for large $x$

$$
I_{n}(x) \approx(2 \pi x)^{-\frac{1}{3}} e^{x} w_{n}(x)+\mathrm{O}\left(e^{-x} x^{-\frac{1}{2}}\right),
$$

fails to be of practical use when $n$ is $\mathrm{O}\left(x^{\frac{1}{3}}\right)$ or $x=\mathrm{O}\left(n^{2}\right)$. He points out also that Nicholson's formula for $I_{n}(x)$ when either $n$ or $x$ is large is not so useful for rapid tabulation as the author seemed to think and so a new formula for $I_{n}(n x)$ is given for the case in which $n$ is large and $x$ has any real value. If $z=\left(1+x^{2}\right)^{3}$

$$
I_{n}(n x) \approx(2 \pi n z)^{-1} e^{n z}[(z-1) / x]^{n} \exp \sum_{m=1} \psi_{m}\left(x^{2}\right)\left(n z^{3}\right)^{-m},
$$

where the coefficients $\psi_{m}\left(x^{2}\right)$ are polynomials whose values are given up to $m=8$, the first two being $24 \psi_{1}\left(x^{2}\right)=3 x^{2}-2,16 \psi_{2}\left(x^{2}\right)=x^{4}-4 x^{2}$. This formula was derived from the asymptotic solution of the Riccatian equation

$$
d y / d x+y / x+y^{2}=n^{2}\left(1+x^{-2}\right)
$$

and is used for example to calculate $I_{100}(75)$.

As an example of series involving special functions other than polynomials multiplied by exponential or trigonometric functions, we may mention Hadamard's expression ${ }^{53}$ for $I_{0}(x)$ as a series whose terms involve 
the error function and polynomials. In Kibble's extension of the formula ${ }^{54}$ use is made of the incomplete gamma function.

The method of stationary phase may give rise to series of special functions but in some cases they seem to.be of the type described by Lanczos in which the coefficients of the powers of $1 / x$ differ from the usual ones. Thus BIJL ${ }^{51}$ finds that

$$
\left|J_{n}(x)-\operatorname{Im}\left\{(1 / \pi) e^{i\left(x-\frac{1}{2} n \pi\right)} \sum_{s=0}^{m-1}\left[G_{2 s} /(2 s) !\right](2 i / x)^{s+\frac{1}{2}} \Gamma\left(s+\frac{1}{2}\right)\right\}\right| \leq C x^{-\frac{1}{2}-\frac{1}{2} m}
$$

where $C$ is a constant depending on $m$ and $G_{0}=1, G_{2 s-1}=0$,

$G_{2 s}=\sum_{r=0}^{2 s-1}[\Gamma(2 s) /\{r ! \Gamma(2 s-r)\}]\left[(d / d y)^{r}(n \tanh n y+i x \sinh y-i x y)\right]_{y=0} G_{2 s-r-1}$.

For the asymptotic expansion of functions related to the Bessel functions reference may be made to the book of Watson and the papers of Meijer. The asymptotic forms of the ber, bei, ker and kei functions are

where

$$
\begin{array}{lll}
\operatorname{ber}(z) \approx(2 \pi z)^{-\frac{1}{2}} e^{\alpha(z)} \cos \beta(z), & & |\arg z|<1 /[4 \pi] \\
\operatorname{bei}(z) \approx(2 \pi z)^{-\frac{1}{2}} e^{\alpha(z)} \sin \beta(z), & & \\
\operatorname{ker}(z) \approx(\pi / 2 z)^{\frac{1}{2}} e^{\alpha(-z)} \cos \beta(-z), & & |\arg z|<5 \pi / 4 \\
\operatorname{kei}(z) \approx(\pi / 2 z)^{\frac{1}{2}} e^{\alpha(-z)} \sin \beta(-z), &
\end{array}
$$

$$
\begin{aligned}
& \alpha(z) \approx z / 2^{\frac{1}{2}}+1 /\left[8 z 2^{\frac{1}{2}}\right]-25 /\left[384 z^{3} 2^{\frac{1}{2}}\right]-13 /\left[128 z^{4}\right]-\cdots, \\
& \beta(z) \approx z / 2^{\frac{1}{2}}-\pi / 8-1 /\left[8 z 2^{\frac{1}{2}}\right]-1 /\left[16 z^{2}\right]-25 /\left[384 z^{3} 2^{\frac{1}{2}}\right]-\cdots .
\end{aligned}
$$

For the Struve function there is the estimate

$$
H_{n}(z) \approx Y_{n}(z)+(1 / \pi) \sum_{m=0}^{p-1} \frac{\Gamma\left(m+\frac{1}{2}\right)}{\Gamma\left(n+\frac{1}{2}-m\right)}\left(\frac{1}{2} z\right)^{n-2 m-1}+\mathrm{O}\left(z^{n-2 p-1}\right) .
$$

When $n$ is real and $z$ positive, the error after $p$ terms in the asymptotic expansion is of the same sign as and numerically less than the first term neglected, provided that

$$
R\left(p-n+\frac{1}{2}\right) \geq 0 \text {. }
$$

\section{F. Series for the zeros of Bessel functions}

Semiconvergent series for the Bessel functions had no sooner been found than they were used to locate the zeros of these functions, particularly those of large order. When the series are truncated, the problem to be solved is essentially that of the solution of the transcendental equation

$$
\tan x=\text { Rational function of } x \text {. }
$$

In the work of CAUCHY ${ }^{5}$ use was made of Lagrange's expansion and series were found for the solution of the equation $\tan x=k x$ for various values of $k$. STOKES ${ }^{55}$ obtained series for $x_{8}$ and $x_{8}{ }^{\prime}$ which are reproduced in Rayleigh's Sound. McMAHoN ${ }^{56}$ extended these series in 1895 and found that if $j_{r, s}$ denotes the $s$-th positive root of $J_{r}(x)=0$, there is a polynomial approximation of type

$$
j_{r, 8} \approx u-m v-m_{1} v^{3}-m_{2} v^{5}-m_{3} v^{7}-\cdots
$$


where

$$
\begin{gathered}
m=4 r^{2}-1, \quad u=\frac{1}{4}(2 r-1+4 s), \quad 8 u v=1 \\
3 m_{1}=4 m(7 m-24), \quad 15 m_{2}=32 m\left(83 m^{2}-816 m+2880\right), \\
105 m_{3}=64 m\left(6949 m^{3}-133088 m^{2}+1298880 m-4838400\right) .
\end{gathered}
$$

For the high roots of $Y^{(r)}(x)=0$, AIREY ${ }^{57}$ gave the estimate

$$
Y_{r s} \approx(2 r+4 s+1) \pi / 4-\arctan [2(\ln 2-\gamma) / \pi]
$$

and an approximate expression for a zero $y_{r, s}$ of $Y_{r}(x)$ is given by the series used for $j_{r, 8}$ but with $u=\frac{1}{4} \pi(2 r+1+4 s)$. Additional terms in the series for $j_{r, s}$ and $y_{r, s}$ have been found by the Bessel function sub-committee of the BAAS and the coefficients have been computed. BICKLEY \& MILLER ${ }^{58}$ have discussed the formula in the reversion of series which is used by McMahon.

McMahon gave also series for the zeros of the derivatives of $J_{r}(x)$, $x^{-\frac{1}{2}} J_{r}(x)$ and $Y_{r}(x)$. These may be derived from the series for a root of the equation

$$
x J_{r+1}(x)=t J_{r}(x)
$$

which may be derived from the differential equation

$$
\left(x^{2}-2 r t+t^{2}\right) d x / d t=x .
$$

The coefficients in the series for $x^{2}$ are simpler than those in the series for $x$, indeed, if $X=\frac{1}{4} \pi(2 r+4 s+1)$

$$
x^{2}=X^{2}+C+C_{1} X^{-2}+C_{2} X^{-4}+C_{3} X^{-6}+\cdots
$$

where

$$
\begin{aligned}
C & =2 t-m, \quad C_{1}=2 r t^{2}-(2 / 3) t^{3}+\frac{1}{2} m-m^{2} / 3, \quad 4 m=(2 r+1)(2 r+3) \\
20 C_{2}=8 t^{5}+(20-40 r) t^{4}+\left(40 r^{2}-80 r-10\right) t^{3} & +40 m r t^{2}-30 m+29 m^{2}-8 m^{3} .
\end{aligned}
$$

This is an extended form of the result given by BATEMaN. ${ }^{69}$ In a letter of August 23, 1943, S. O. Rice of the Bell Telephone Laboratories extended the result by a series suitable for large values of $t$. If $t=1 / z$ and $w=1-2 r z$, $Y=\frac{1}{4} \pi(2 r+3+4 s)$ the new series is

$x^{2}=Y^{2} w^{1 / r}+Y^{4} w^{2 / r-1} \frac{1-2 z(r-1)-2 z^{2}(r-1)-w^{1-1 / r}}{2(r-1)}+Y^{6}()+\cdots$

so that roughly

$$
x^{2} \approx Y^{2}\left[w^{1 / r}+(2 / 3) Y^{2} z^{3} w^{2 / r-1}\right] .
$$

This formula has been tested for $x \geq 13$ by means of a graph of $x^{2}$ against $t$. Since

$$
J_{r+1}(x)=(r / x) J_{r}(x)-J_{r}^{\prime}(x),
$$

the equation for $x$ can also be written in the form

$$
(r-t) J_{r}(x)=x J_{r}^{\prime}(x) .
$$


When $J_{r}(x)$ is replaced by $Y_{r}(x)$ the series for $x^{2}$ can still be used with appropriate values for $X$ and $Y$. This is true also when a function of type $J_{r}(x) \cos a+Y_{r}(x) \sin a$ is used instead of $J_{r}(x)$.

McMahon remarks that his series for the zeros of $J_{r}^{\prime}(x)$ and of the derivative of $x^{-1} J_{r}(x)$ give respectively the roots of $\tan x=2 x$ and of the equation of RAYLEIGH (Sound, v. 2, p. 232-3)

$$
\left(2-x^{2}\right) \tan x=2 x .
$$

A series which generalizes the second of these series and also Cauchy's series for a solution of Poisson's equation

$$
\tan x=9 x /\left(9-4 x^{2}\right)
$$

gives the solution of $\tan x=x /(a-c x)$ in the form

$$
\begin{aligned}
x= & s \pi-z-(c+d-1 / 3) z^{3}-\left(d^{2}+4 c d+2 c^{2}-d-4 c / 3+1 / 5\right) z^{5} \\
& -\left(d^{3}+9 d^{2} c-2 d^{2}+15 d c^{2}+5 c^{3}-8 c d+d-5 c^{2}+23 c\right. \\
& -1 / 7) z^{7}+\cdots,
\end{aligned}
$$

where $d=a c, z=1 /(\operatorname{cs} \pi)$. For the equation $\tan x=a x$, Cauchy gave a series for the case $a=1$ which is not given directly by putting $c=0$ in the foregoing series. If $S=\left(s+\frac{1}{2}\right) \pi$, Cauchy's series is

$$
x=S-S^{-1}-(2 / 3) S^{-3}-(13 / 15) S^{-5}-(146 / 105) S^{-7}-\cdots
$$

For the roots of $2 x J_{r}^{\prime}(x)=J_{r}(x)$, McMahon gave the series

where

$$
x=X-M_{1} V-M_{2} V^{3}-M_{3} V^{5}-M_{4} V^{7}-\cdots
$$

$$
\begin{aligned}
M & =4 r^{2}, \quad 8 V X=1, \quad M_{1}=M+7, \quad 3 M_{2}=4\left(7 M^{2}+154 M+95\right), \\
15 M_{3} & =32\left(83 M^{3}+3535 M^{2}+3561 M+6133\right), \quad 105 M_{4}=64 .
\end{aligned}
$$

For the $s$-th positive root of the equation

$$
J_{n}(x) Y_{n}(k x)=Y_{n}(x) J_{n}(k x), \quad k>1, \quad M=4 n^{2},
$$

McMahon gave the series

where

$$
x \approx b+p c+\left(q-p^{2}\right) c^{3}+\left(r-4 p q+2 p^{3}\right) c^{5}+\cdots
$$

$$
\begin{gathered}
b c=1, \quad(k-1) b=s, \quad 8 k p=M-1, \\
3(8 k)^{3}(k-1) q=4(M-1)(M-25)\left(k^{3}-1\right) \\
5(8 k)^{5}(k-1)=32(M-1)\left(M^{2}-1\right)\left(M^{2}-114 M+1073\right) .
\end{gathered}
$$

McMahon also gave a similar series for the roots of

$$
J_{n}^{\prime}(x) Y_{n}^{\prime}\left(k^{\prime} x\right)=Y_{n}^{\prime}(x) J_{n}^{\prime}\left(k^{\prime} x\right), \quad k k^{\prime}=1,
$$

the coefficients being now

$$
\begin{aligned}
& p=(M+3) / 8 k, q=4\left(M^{2}+46 M-63\right)\left(k^{3}-1\right) /\left[3(8 k)^{3}(k-1)\right] \\
& r=32\left(M^{3}+185 M^{2}-2053 M+1899\right)\left(k^{5}-1\right) /\left[5(8 k)^{5}(k-1)\right] .
\end{aligned}
$$


SASAKI ${ }^{60}$ has given a series for the roots of $Y_{n}(k x) J_{n}{ }^{\prime}(k a)=Y_{n}(k a) J_{n}{ }^{\prime}(k x)$, and FISCHER ${ }^{61}$ has given a series for the roots of $J_{1}(x) Y_{0}(k x)=Y_{1}(x) J_{0}(k x)$ using McMahon's method.

For the $s$-th positive zero of ber $x$, bei $x, \operatorname{ker} x$ and kei $x$, AIREy ${ }^{62}$ has given the series

$$
x \approx b+B+4 \sqrt{2} B^{2}+76 B^{3} / 3-96 \sqrt{2} B^{4}-124768 B^{5} / 15-\cdots
$$

where the values of $b$ are respectively $(s-3 / 8) \pi \sqrt{2},(s+1 / 8) \pi \sqrt{2}$, $(s-5 / 8) \pi \sqrt{2},(s-1 / 8) \pi \sqrt{2}$ and $8 b B=1$.

For the zeros of $\operatorname{ber}^{\prime} x, \operatorname{bei}^{\prime} x, \operatorname{ker}^{\prime} x$ and $\operatorname{kei}^{\prime} x$ the series given by AIREY ${ }^{62}$ is

$$
x \approx c-3 C-12 \sqrt{2} C^{2}-156 C^{3}-864 \sqrt{2} C^{4}-19872 C^{5} / 5-\cdots
$$

where $8 c C=1$ and the respective values of $c$ are $(s+3 / 8) \pi \sqrt{2},(s-1 / 8) \pi \sqrt{2}$, $(s-3 / 8) \pi \sqrt{2},(s+1 / 8) \pi \sqrt{2}$.

McMahon's series for $j_{r, s}$ is given in an abbreviated form in Rayleigh's Sound (v. 1, p. 329) and in a complete but incorrect form in Gray and Mathews Bessel Functions. The error is noted by Marshall. ${ }^{63}$ Smith, RoDGERS \& TRAUB ${ }^{64}$ say that McMahon's series for the zeros of the first derivative of $J_{r}(x)$ gives the $(s+1)$-th root and not the $s$-th root.

In 1918 WATSON ${ }^{65}$ noted some cases of failure of McMahon's formula for $j_{r, s}$, particularly for large values of $r$. For such values Debye's asymptotic formula

$$
\begin{gathered}
J_{n}(n \sec b) \approx\left(\frac{1}{2} n \pi \tan b\right)^{-\frac{1}{1}}\left(\cos B+\frac{3+5 \cot ^{2} b}{24 n} \cot b \sin B+\cdots\right), \\
B=n(\tan b-b)-\frac{1}{4} \pi
\end{gathered}
$$

may be used except when $b$ is very small. In this case Watson recommends the formula

$$
\begin{gathered}
J_{n}(n \sec b) \approx(2 / 3) \tan b\left[\cos T J_{-1 / 3}(U)+\cos T^{\prime} J_{1 / 3}(U)\right] \\
3 T=n\left(3 \tan b-\tan ^{3} b-3 b-\pi\right), \quad 3 T^{\prime}=3 T+2 \pi, \quad 3 U=n \tan ^{3} b
\end{gathered}
$$

which gives an error less than $24 / n$. Other formulae may be obtained by assuming a definite order of magnitude for $x-n$. In 1899 KOPPE ${ }^{66}$ used the form $x=u^{3}+u v$, where $u^{3}=n$ and appropriate asymptotic forms were developed by WATSON ${ }^{67}$ and Fok. ${ }^{68}$ These like the formula just given may be expressed most conveniently in terms of Bessel functions of orders $\pm 1 / 3$ and are then valid not only when $n / x$ is close to 1 but also in the domain of validity of Debye's expressions. When

$$
|x-n| \ll n^{3 / 5}
$$

the expressions of Watson and of Fok are equivalent to the formulae involving the integral of Airy.

In 1922 AIREY ${ }^{69}$ gave a formula of type

$$
j_{r, 8} \approx t^{3}+a_{1}(s) t+b_{1}(s) t^{-1}, \quad t^{3}=r, \quad s=1,2,3 .
$$


The improvement of this formula may require the use of other estimates of $J_{n}(x)$ than those just mentioned. Those of Bijl and Lehmer may be worth studying in this connection.

For equations which involve squares and products of Bessel functions asymptotic expansions of these functions are needed. For these reference may be made to p. 223 of the book of Watson and to the paper of BAILEY ${ }^{70}$ If

$$
\begin{array}{ll}
P=J_{m} J_{n}+Y_{m} Y_{n}, & Q=J_{m} Y_{n}-J_{n} Y_{m}, \\
R=J_{m} J_{n}-Y_{m} Y_{n}, & S=J_{m} Y_{n}+J_{n} Y_{m},
\end{array}
$$

where the argument of the functions is $z$ and if $2 c=m-n, 2 s=m+n$, $4 z=m+n+2 a, 2 t=(m-n) \pi, w z=1$, Bailey's series are

$$
\begin{aligned}
& P-Q \cot t \approx\left(m^{2}-n^{2}\right)(w \operatorname{cosec} t)_{4} F_{1}\left(1+s, 1+c, 1-s, 1-c ; 3 / 2 ;-w^{2}\right), \\
& P+Q \tan t \approx(2 / \pi) w \cos t_{4} F_{1}\left(\frac{1}{2}+s, \frac{1}{2}+c, \frac{1}{2}-s, \frac{1}{2}-c ; \frac{1}{2} ;-w^{2}\right), \\
& R \sin a-S \cos a \approx(2 / \pi) w \sum_{p=0}^{\infty}\left(-\frac{1}{4} w^{2}\right)^{p} \frac{\left(\frac{1}{2}+m\right)_{2 p}\left(\frac{1}{2}-m\right)_{2 p}}{(2 p) !} F^{\prime}, \\
& R \cos a+S \sin a \approx-(1 / \pi) w^{2} \sum_{p=0}^{\infty}\left(-\frac{1}{4} w^{2}\right)^{p} \frac{\left(\frac{1}{2}+m\right)_{2 p+1}\left(\frac{1}{2}-m\right)_{2 p+1}}{(2 p+1) !} F^{\prime \prime},
\end{aligned}
$$

where

$$
\begin{aligned}
& F^{\prime}={ }_{3} F_{2}\left(\frac{1}{2}+n, \frac{1}{2}-n,-2 p ; \frac{1}{2}-m-2 p, \frac{1}{2}+m-2 p ;-1\right), \\
& F^{\prime \prime}={ }_{3} F_{2}\left(\frac{1}{2}+n, \frac{1}{2}-n,-2 p-1 ;-\frac{1}{2} m-2 p, m-\frac{1}{2}-2 p ;-1\right) .
\end{aligned}
$$

Since asymptotic expansions of Bessel functions are not generally useful for the determination of the small roots of equations, other methods have been devised. Some of these have been used by AIREY. ${ }^{71}$ IKEDA ${ }^{72}$ strongly recommends a method based on the use of the series

$$
\begin{gathered}
J_{n}(a x)=a^{n} \sum_{s=0}^{m-1} \frac{\left(1-a^{2}\right)^{s}}{s !}(x / 2)^{s} J_{n+8}(x)+a^{n} \frac{\left(1-a^{2}\right)^{m}}{m !}(x / 2)^{m} \frac{J_{n+m}(\theta x)}{\theta^{n+m}}, \\
0<\theta<1,
\end{gathered}
$$

and when $x$ differs very little from a zero of $J_{n}(z)$, he finds the approximate value of this zero to be

$$
x+\Delta+\frac{2 n+1}{2 x} \Delta^{2}+\left\{\frac{(4 n+1)(2 n+1)}{2 x^{2}}-1\right\} \frac{\Delta^{3}}{3},
$$

where $\Delta=J_{n}(x) / J_{n+1}(x)$.

1 T. J. I'A. Bromwich, Infinite Series, London, Macmillan, 1908, p. 322-338. For the history of the Euler-Maclaurin sum formula see E. W. Barnes. London Math. So. Proc., s. 2 , v. 3, 1905, p. 253-272.

${ }^{2}$ H. PoInCARE, Acta Math., v. 8, 1886, p. 296

${ }^{3}$ C. G. J. J ACOBI, Ast. Nach., v. 28, 1848, col. 94; Werke, v. 7, p. 174.

4 S. D. Poisson, École Polytechnique, Paris, J., v. 12, 1823, p. 349.

$\checkmark$ A. Cauchy, Inst. de France, Acad. d. sci., Mémoires divers savans, v. 1, 1827, p. 272 Oeuvres, s. 1, v. 1, p. 277-278.

- E. W. BARnes, London Math. So., Proc., s. 2, v. 5, 1907, p. 59-116.

7 J. Ivory, R. So. London, Trans., v. 113, 1823, p. 409-495, v. 128, 1838, p. 170-229. 
${ }^{8} \mathrm{G}$. N. Watson, A Treatise on the Theory of Bessel Functions, Cambridge 1922, p. 207-210.

${ }^{9}$ N. S. Koshliakov, London Math. So., J., v. 4, 1929, p. 297-299.

${ }^{10}$ B. Strogonoff, Inst. Math. Stekloff, Travaux, v. 9, 1935, p. 223-233.

11 J. R. Airey, Archiv. Math. Phys., v. 22, 1914, p. 30, B.A.A.S., Report, 1913 p. 115. Phil. Mag., s. 7, v. 24, 1937, p. 521-552.

i2 A. Lodge, B.A.A.S., Report, 1906, p. 494-498, 1907, p. 95-97.

${ }^{13}$ N. Nielsen, Handbuch der Theorie der Cylinderfunktionen, Leipzig, 1904.

14 D. Burnetr, Cambridge Phil. So., Proc., v. 26, 1930, p. 145-151.

${ }^{15}$ L. V. KING, R. So. London, Trans., v. 214A, 1914, p. 373-432.

${ }^{16} \mathrm{~K}$. Weierstrass, Akad. d. Wissen. Berlin, Sitzungsb. 1885, p. 633-639, 789-805, Werke v. 3, Berlin, 1903, p. 1-37.

${ }_{17}$ G. G. Srokes, Cambridge Phil. So. Trans., v. 10, 1857, p. 106-128, Math. \& Phys. Papers, v. 4, p. 77-109.

${ }_{18}$ H. Hankel, Math. Ann., v. 1, 1869, p. 467-501.

19 C. Lanczos, J. Math. Phys., M.I.T., v. 17, 1938, p. 123-199.

${ }^{20} \mathrm{~J}$. Liouville, J. de Math., s. 1, v. 2, 1837, p. 16-35.

21 A. KNeser, Math. Ann., v. 49, 1897, p. 383-399.

22 J. HoRN, Math. Ann., v. 52, 1899, p. 271-292, 342-362.

${ }^{23}$ G. D. Birkhoff, Am. Math. So. Trans., v. 9, 1908, p. 219-231.

${ }^{24}$ E. W. Hobson, London Math. So. Proc., s. 2, v. 6, 1908, p. 374-388.

${ }^{25}$ G. HoHeISEL, J.f.d. reine u. angew. Math., v. 153, 1924, p. 228-244.

26 Y. IkedA, Tôhoku Math. J., v. 29, 1928, p. 284-290.

${ }^{27}$ H. Jeffreys, London Math. So., Proc., s. 2, v. 23, 1925, p. 428-436, Phil. Mag., s. 7, v. 33,1942 , p. $451-456$.

${ }^{28}$ L. Brillouin, J. de Phys., le Radium, v. 7, 1926, p. 353-368, Inst. de France, Acad. des Sci. Comptes Rendus, v. 183, 1926, p. 24-26.

${ }^{29}$ H. A. Kramers, $Z$. $f$. Physik, v. 39, 1926, p. 828-840.

${ }^{30}$ G. Wentzel, $Z$. $f$. Physik, v. 38, 1926, p. 518-529.

31 S. Goldstein, London Math. So., Proc., v. 28, 1928, p. 81-101, v. 33, 1932, p. 246-252.

${ }^{32}$ G. N. Watson, R. So. London, Proc., v. 94A, 1918, p. 190-206.

${ }^{33} \mathrm{~F}$. CARLINI, Ricerche sulla convergenza della serie che serva alla soluzione del problema

di Keplero, Milan, 1817, 48 p. Translation into German in Jacobi's Werke, v. 7, p. 184-245. ${ }^{34}$ C. G. J. J ACOBI, Ast. Nach., v. 28, 1848, col. 257-270, Werke, v. 7, p. 175-188.

${ }^{35}$ P. DeBYe, Math. Ann., v. 67, 1909, p. 535-558, Bayerisch. Akad. d. Wissen, Sitzungb., v. 40,1910 , no. 5,29 p.

${ }^{36}$ G. N. Watson, Cambridge Phil. So., Proc., v. 19, 1916-1919, p. 42-48, 96-110;

Phil. Mag., s. 6, v. 35, 1918, p. 369; Bessel Functions, Ch. 8.

${ }^{37}$ S. C. Van Veen', Math. Ann., v. 97, 1927, p. 696-710.

${ }^{38}$ F. Pollaczek, Annalen d. Physik, s. 5, v. 2, 1929, p. 991-1011.

${ }^{39}$ R. E. Langer, Am. Math. So., Trans., v. 33, 1931, p. 23-64, v. 34, 1932, p. 447-480.

${ }^{40}$ E. JAHNKe \& F. Emde, Tables of Functions, 3rd. edition, Leipzig \& Berlin, 1938.

${ }^{41}$ F. EMDE, Zeit. angew. Math. Mech., v. 17, 1937, p. 324-340.

${ }^{42}$ F. Emde \& R. RüHLe, Deutsch. Math. Verein, Jahresb., v. 43, 1934, p. 251-270.

${ }_{43}$ J. R. Airey, Phil. Mag., s. 7, v. 24, 1937, p. 521-552.

${ }_{44}$ J. W. Nicholson, Phil. Mag., s. 6, v. 14, 1907, p. 697-707; v. 16, 1908, p. 271-275;

see also A. ERDElyi, Časopis Mat. a Fys., v. 67, 1937, p. 240-248.

${ }^{45}$ Rayleigh, Phil. Mag., s. 6, v. 20, 1910, p. 1001-1004, Scientific Papers, v. 5, p. $617-620$.

${ }^{46}$ C. S. Meijer, Akad. v. Wetensch., Amsterdam, Proc., v. 35, 1932, p. 656-667,

853-866, 948-968, 1079-1090, 1291-1303; Math. Ann., v. 108, 1933, p. 321-359.

${ }^{47}$ V. A. For, Acad. d. sci., U.R.S.S., Comptes Rendus, new series, v. 1, 1934, p. 99-102.

18 A. Svetlov, Acad. d. sci., U.R.S.S., Comptes Rendus, new series, v. 1, 1934, p. 445-448.

49 S. C. VAN VeEn, Zentralblatt f. Math., v. 8, 1934, p. 259.

60 J. G. VAN DER CORPUT, Compositio Math., v. 1, 1934, p. 15-38, v. 3, 1936, p. 328-372. s1 J. Bijl, Nieuw Archief v. Wiskunde, v. 19, 1936, p. 63-85, Diss. Groningen, 1937, 106 p. ${ }^{62}$ D. H. Lehmer, $M T A C$, v. 1, No. 5, 1944, p. 133-135.

${ }^{63}$ J. Hadamard, Math. So. France, Bull., v. 36, 1908, p. 77-85, Watson's Bessel Functions, p. 204-205.

${ }_{64}$ W. F. KIBBLE, Indian Math. So., J. v. 3, 1939, p. 271-294.

${ }^{65}$ G. G. Stokes, Cambridge Phil. So., Trans., v. 9, 1850, p. 184, 186. Math. \& Phys.

Papers, v. 2, Cambridge, 1883 , p. $353,355$.

${ }_{66} \mathrm{~J}$. McMAHON, Annal.s Math., s. 1, v. 9, 1895, p. 23-30.

b7 J. R. Airey, Phys. So. London, Proc., v. 23, 1911, p. 221.

68 W. G. Bickley \& J. C. P. Miller, Phil. Mag., s. 7, v. 34, 1943, p. 35-36.

${ }^{69}$ H. Bateman, National Math. Mag., v. 18, 1943, p. 10-11.

${ }^{\circ} \mathrm{L}$. SASAKI, Tôhoku Math. J., v. 5, 1914, p. 45-47.

61 J. FISCHER, Ingenieur Archiv, v. 10, 1939, p. 95-112. 
62 J. R. Airey, B.A.A.S., Report, 1927, p. 253-254.

${ }^{63}$ W. Marshall, Annals Math., s. 2, v. 11, 1910, p. 153-160.

64 D. B. Smith, L. M. Rodgers \& E. H. TrauB, Franklin Inst., J., v. 237, 1944, p. 301-303.

${ }_{65}^{6}$ G. N. Watson, R. So. London, Proc., 94A, 1918, p. 190-206.

${ }^{66} \mathrm{~W}$. KoPPE, Die Ausbreitung einer Erschütterung an den Wellenmaschinen, (Program no. 96, Andreas Realgymn. Berlin) Berlin, 1899, 28p. 1 table. See also J. W. Nicholson, Phil. Mag., s. 6, v. 16, 1908, p. 271, v. 18, 1909, p. 6.

${ }^{67} \mathrm{G}$. N. Watson, Bessel Functions, p. 248-252.

68 V. A. FoK, loc. cit., no. 47.

${ }^{69}$ J. R. AIREY, B.A.A.S., Report, 1922, p. 271-272, JAHNKe \& EMDE 15, p. 143.

70 W. N. BaILEY, London Math. So., J., v. 4, 1929, p. 118-120.

${ }^{71}$ J. R. Alrey, Phil. Mag., s. 6, v. 31, 1916, p. 520-528, v. 32, 1916, p. 7-14, v. 41, 1921, p. 200-205, B.A.A'S. Report, 1927 , p. 252.

72 Y. IKEDA, Zeit. Angew. Math. Mech., v. 5, 1925, p. 80-83.

\section{INDEX OF AUTHORS REFERRED TO IN INTRODUCTIONS, I-XII, AND IN XIII}

Adams, E. P., IV

AIREY, J. R., II, VII, VIII, IX, XIII

AIRY, G. B., I, V, VI, XIII

ALdIS, W. S., II

ANDing, E., III

ANSELl, P. R., VIII

ARChibald, R. C., $\mathrm{X}$

BaIley, W. N., XIII

BARNES, E. W., XIII

BASSET, A. B., III

Bateman, H., XIII

Bernoulli, D., I, II

Bernoulli, J., V, XIII

Bessel, F. W., I

BICKLEY, W. G., X, XIII

BIJL, J., XIII

BIRKHOFF, G. D., XIII

BôCHER, M., I, II

BOOLE, G., XIII

Brasey, E., XI

Brauer, E., IX

BRAUER, P., IX

BrILlouin, L., XIII

BROMWICH, T. J. I'A., XIII

BuRnetT, D., XIII

Callandreau, O., XIII

Carlini, F., XIII

Cauchy, A., XII, XIII

Chapman, S., I

Clifford, W. K., VII

Costello, J. B., XI

Debye, P., XIII

Doodson, A. T., IV

Dubors, F., XI

EMde, F., II, III, XIII

ERDÉLYI, A., XIII

EULER, L., I-IV, VII, X, XIII

FISCHER, J., VI, XIII
FISHER, R. A., VIII

For, V. A., XIII

Gilbert, P., X, XII

GoldsteIN, S., IX, XIII

Gray, A., II, XIII

Greenhill, G., VII

GREGORY, W., XIII

HADAMARD, J., XIII

HAMY, M., VII

HANKel, H., II, III, XIII

HANSEN, P. A., I

HARDY, G. H., V, VI

HAURVITZ, E., IV

HAVELOCK, T. H., X

Heatley, A. H., VI

Heaviside, O., II

HeINE, H. E., II

HILL, M. J. M., XIII

Hobson, E. W., XIII

HoHeISEL, G., XIII

Hollister, S. C., XI

HoRN, J., XIII

IKEDA, Y., XIII

IVORY, J., III, XIII

JACOBI, C. G. J., XIII

JAHNKE, E., II, III, XIII

JEFFREYS, H., V, XIII

KeLvin, XI

KenNelly, A. E., XI

KIbBLE, W. F., XIII

KING, L. V., X, XIII

KIRChHOFF, G., III

KNESER, A., XIII

KOPPE, W., XIII

Koshliakov, N. S., XIII

Kramers, H. A., XIII

KumMer, E. E., III

KÜSSNER, H. G., X 
LAGRANGE, J. L., I

LAMB, H., IV

Lanczos, C., XIII

LANGER, R. E., XIII

LAws, F. A., XI

LEGENDRE, A. M., XIII

LEHMER, D. H., XIII

Leibniz, G. W., V

LIOUVILLE, J., XIII

Lissajous, J. A., IV

LODGE, A., IV, XIII

LOMMEL, E. C. J. v., I, IV, IX, XII

MACLAURIN, C., XIII

MAcRobert, T. M., II

MAGGI, G. A., I

Marshall, W., XIII

Mascart, É. E. N., I, VI

Mathews, G. B., II, XIII

Mayall, R. H. D., XII

MCLACHLAN, N. W., XI

McMahoN, J., II, XIII

MeIJer, C. S., XIII

MeYers, A. L., XI

Michell, J. H., XI

Michelson, A. A., VII

MIE, G., IV

MilleR, J. C. P., V, XI, XIII

DE MoIvre, A., XIII

MORSE, P. M., IV

Moulton, F. R., VII

NAYLER, J., $\mathrm{X}$

NeumanN, C. G., II, XI

NYMTP, IV, XI

Nicholson, J. W., IX, XIII

Nielsen, N., III, IX, XIII

O'ToOle, A. L., VI

OWEN, S. P., IX

Paris, E. T., IV

Pease, F. G., VII

Peters, $X$

PIERCE, P. H., XI
Poincare, H., XIII

PoIsson, S. D., VIII, XIII

Pollaczek, F., XIII

RAYLEIGH, IV, V, VI, IX, XIII

RICCATI, V., XIII

RICE, S. O., XIII

RODGERs, L. M., XIII

RÜHLE, R., XIII

Russell, A., XI

SASARI, L., XIII

SAvidge, H. G., XI

SchafHeItLIN, P., IV, V, VIII

SCHLÄFLI, L., II, IX

SCHLÖMILCH, O., I

Schumann, T. E. W., VII

SCHWARZ, L., $X$

SCHWERD, F. M., I

SEebeck, L., IV

SEELIGer, $H ., X$

SMITH, B. A., II, XI

SMITH, D. B., XIII

Stern, M. A., IV

StieltJes, T. J., XIII

STIRLING, J., XIII

Stokes, G. G., VI, VIII, XI, XIII

Strogonoff, B., XIII

Struve, H., IX, XII, XIII

Svetlov, A., XIII

TAYLOR, B., XIII

TODHUNTER, I., III

TöLKE, F., XI

Traub, E. H., XIII

VAN DER CoRput, J. G., XIII

Van Veen, S. C., XIII

WANNIER, G., IV

Watson, G. N., II, IV, V, IX, XIII

WEBER, H., II, IX

Weierstrass, K. T. W., XIII

Wentzel, G., XIII

WhITEHEAD, C. S., XI 


\section{PART II \\ Bibliography of \\ Authors of Tables and Graphs}

M. Abramowitz, see Lowan

E. P. ADAMS

1. Smithsonian Mathematical Formulae ... (Smithsonian Miscellaneous Collections, v. 74, no. 1.) Washington, 1922; first reprint with corrections, 1939, p. 84-86. [IV].

J. R. Airey (see also WebB)

1. Phys. So. London, Proc., v. 23, 1911, p. 221-224. [II].

Errata: p. 223, $y_{0,2}$ for 3.95769 , read $3.95768 ; y_{1,1}$, for 2.19685 , read 2.19714 . BAASMTC 1, p. 201.

2. Phys. So. London, Proc., v. 23, 1911, p. 227, 231-232. [III]. Part of the table on p. 227 is reproduced by D. WRINCH.

3. Phil. Mag., s. 6, v. 21, 1911, p. 742. [II].

4. Phil. Mag., s. 6, v. 22, 1911, p. 660-663. [II].

4. B.A.A.S., Report, 1911, p. 75-78.

5. Arch. Math. Phys., s. 3, v. 20, 1913, p. 291-294. [I, II].

6. B.A.A.S., Report, 1913, p. 87, 115-130. [II].

7. Arch. Math. Phys., s. 3, v. 22, 1913, p. 30-43. [II].

8. B.A.A.S., Report, 1914, p. 75-82. Greatest error 10 $0^{-7}$. [II].

9. B.A.A.S., Report, 1914, p. 83-86. [II].

10. B.A.A.S., Report, 1915, p. 28-32. Portion of the table to $6 \mathrm{D}$ based on a ms. to $10 \mathrm{D}$, and the other portion to $10 \mathrm{D}$ based on ms. to $12 \mathrm{D}$. [I].

11. B.A.A.S., Report, 1915, p. 32-33. [II].

12. B.A.A.S., Report, 1915, p. 34-36. [II].

13A. B.A.A.S., Report, 1916, p. 92-96. [I, II].

13B. Phil. Mag., s. 6, v. 31, 1916, p. 521, 523, 525, 527. [I, II].

14. Phil. Mag., s. 6, v. 32, 1916, p. 11-14. [I, II].

15. Phil. Mag., s. 6, v. 34, 1917, p. 193-195. [IV, VI].

16. R. So. London, Proc., v. 94 A, 1918, p. 312. [IX].

17. Phil. Mag., s. 6, v. 36, 1918, p. 241-242. [I, II]. Part of the table on p. 241 is abridged from WilLson and PEIRCE.

18. Phil. Mag., s. 6, v. 41, 1921, p. 200-205. [V, VI]. Partly reprinted in Gray \& MatheWS $1_{2}$, T. XIII, p. 317.

19. B.A.A.S., Report, 1922, p. 271-272. [I].

Errata: Comparison with Watson 3, p. 749f, suggests that the end-figures for $j_{2,1}$ and $j_{1,8}$ should each be increased by unity.

20. B.A.A.S., Report, 1923, p. 290-293. [I, II, IV, VI, IX].

21. B.A.A.S., Report, 1924, p. 279-287. [IX].

22. B.A.A.S., Report, 1924, p. 287-295. [VII].

23. B.A.A.S., Report, 1925, p. 221-233. [IV].

24. B.A.A.S., Report, 1925, p. 234-242. [IV].

25. B.A.A.S., Report, 1925, p. 243-253. [IX].

26. B.A.A.S., Report, 1926, p. 273-275, 297. [X].

27. B.A.A.S., Report, 1927, p. 231. [III, VII].

28. B.A.A.S., Report, 1927, p. 252-253. [VI].

29. B.A.A.S., Report, 1927, p. 254. [XI]. 
30. B.A.A.S., Report, 1928 , p. 319-323. [VIII].

31. Phil. Mag., s. 7, v. 19, 1935, p. 233-235. [I, IV, VI].

32. Phil. Mag., s. 7, v. 19, 1935, p. 236-243. [VIII].

33. Phil. Mag., s. 7, v. 24, 1937, p. 546. [VI].

34. Ms. $I_{0}{ }^{\prime}\left(x i^{i}\right) G_{0}{ }^{\prime}\left(x i^{3}\right)=V_{r}(x)+i V_{u}(x)$, for $x=[0(.02) 10(.05) 11(.1) 12.5(.5) 16(1) 20$; 8D], in possession of L. J. ComRIE. According to B.A.A.S., Report, 1929, p. 251, there were "in course of construction" "the first ten zeros of these functions, six places." $[\mathrm{XI}]$.

G. B. AIRY

1. Mathematical Tracts on the Lunar and Planetary Theories, the Figure of the Earth, Precession and Nutation, the Calculus of Variations, and the Undulatory Theory of Optics, Cambridge, second ed., 1831, p. 410 [tables of $S(u), C(u)$ ], [X].

12. Third ed., Cambridge, 1842, p. 390.

1. Fourth ed., Cambridge, 1858, p. 399; 400 [table of $\Lambda_{1}(x)=2 J_{1}(x) / x$, not in earlier editions]. [I, X].

2. Cambridge Phil. So., Trans., v. 5, 1835, p. 291. [1].

2. Annalen d. Physik, v. 45, 1838, p. 95.

2. AIRY $1_{2}, 1858$, p. 400 .

2. MASCART, 1889 , p. $310 ; \Lambda_{1}(x)$ and $\Lambda_{1}^{2}(x)$.

2. Pernter, 1906, p. 440.

2. HUMPHREYS, 1919-1940.

3. Cambridge Phil. So., Trans., v. 6, 1838, p. 390, 402. [V]. Five and seven-place tables of $A(m)$ for $m=-4(.2)+4$.

32. Annalen d. Physik, Ergänzungsband, v. 1, 1842, p. 245-246. German translation; 5-place tables of $A(m)$ and $A^{2}(m)$.

Errata: See under no. $\mathbf{5}_{\mathbf{2}}$.

4. Phil. Mag., s. 3, v. 18, 1841, p. 7. [I]. 4-place table based on 6-place calculations. Errata: "Errors at 7.4 and 8.0," Liverpool Index.

5. Cambridge Phil. So., Trans., v. 8, 1849, p. 598-599. [V]. Five-place table of $A(m)$ for $m=-5.6(.2)+5.6$.

52. HoGNer, 1923, p. 37, graph p. 38.

Errata (note by J. C. P. MILLER): The writer has recomputed all these values [in the tables of nos. $3_{1}$ and $5_{1}$ ] and finds that, in units of the 7th decimal, the greatest error in any of Airy's 7-decimal values for $-4 \leq m \leq+2$ is \pm 52 units; the error then changes fairly steadily from -56 units at $m=2.2$ to -272 units at $m=3.4$; the final three errors are $-23,+858$, and +2661 of these units. Airy's revised table [no. $5_{1}$ ] has only one error of more than 2 units in the 5 th decimal, the value for $m=-3.6$ is 3 units in error, that given in the original table being, however, correct to five decimals.

W. S. Aldis (see also BiSACRE)

1. R. So. London, Proc., v. 64, 1899, p. 218-223. [III]. See BAASMTC 1, IsHerwood.

12. Gray \& MATHEWS $1_{2}$, p. 313-315.

2. R. So. London, Proc., v. 66,1900 , p. 40-43. [I, II, XI].

P. L. ALGER

1. B.A.A.S., Report, 1923, p. 295-298. [XI]. See Dwight 4.

E. ANDing

1. Sechsstellige Tafeln der Bessel'schen Funktionen imaginären Argumentes, Leipzig, Engelmann, 1911, iv, 72 p.; p. 45-72. [III].

H. D. Arnett, see Colwell

H. BackHaUs

1. Naturwissen., v. 19,1929 , p. 836 . [IV].

2. Annalen d. Physik, s. 5, v. 5, 1930, p. 18-19. [XII]. 
M. BADELlino

1. Rome, Università, sem. mat., Rendiconti, s. 4, v. 3, 1939, p. 279. [III]. There were curiously incorrect abstracts of this paper in both Zentralblatt f. Math., v. 22, 1940, p. 376, and Math. Reviews, v. 2, 1941, p. 45. Our correction of the latter (v. 4, 1943, p. 340) shows that the tables here are of $e^{-x} I_{0}(x), e^{-x} I_{1}(x), e^{x} K_{0}(x), e^{x} K_{1}(x)$, for $x=[20(1) 50 ; 9 D]$.

12. Consiglio naz. d. Ricerche, Istituto appl. d. Calcolo, Pubblicazioni, Rome, s. 2, no. 68,1939 , p. 9 .

S. Bailantine

1. Phys. Rev., s. 2, v. 32, 1928, p. 991. [XII].

D. BANCROFT

1. Phys. Rev., s. 2, v. 59, 1941, p. 589-590. [I].

D. R. Bates \& H. S. W. Massey

1. R. So. London, Proc., v. 177A, 1941, p. 340. [VII].

J. M. Bates

1. Iowa State College, J. Sci., v. 12, 1938, p. 474. [V].

W. P. Berggren, see R. L. Perry

S. Bertram

1. J. Applied Physics, v. 13, 1942, p. 498-500. [I, XII].

F. W. Bessel (see also P. A. Hansen)

1. Akad. d. Wissen., Berlin, Math. Kl., Abh. aus dem Jahre 1824, Berlin, 1826, p. 46-52. [I].

1.. Abhandlungen, v. 1, Leipzig, 1875, p. 103-109.

Errata: A comparison by J. W. WRENCH, Jr., of Bessel's 10-place tables of $J_{0}(x)$ and $J_{1}(x)$ with those in BAASMTC 1 revealed only 3 cases of errors of more than a unit in the last decimal place. Two of these were obviously printer's errors, namely: (1) $J_{0}(1.69)$, for .4037595307 , read .4037595370 -the correct ending figures being 69; (2) $J_{0}(3.10)$, for -.2920634377 , read -.2920643477 . The third error was in $J_{1}(2.42)$, for .5158326995 , read .51583 26997. There were also 45 unit errors in the tenth place. All these 48 errors occur also in $1_{2}$, with an additional error $J_{0}(0.00)=0$. Mr. Wrench found also 23 errors in the columns of first and second differences corresponding to the values published. Apart from 8 unit errors in the last figures of first and second differences for $J_{1}(x)$ there were the following 15 errors of more than a unit in $\Delta$ and $\delta^{2}$ for $J_{0}(x)$ and $J_{1}(x): \Delta, J_{0}(.32, .33)$, for -16036343 , read $-16036341 ; J_{0}(1.06,1.07)$, for -46048657 , read $-46048655 ; J_{0}(2.43,2.44)$, for -51257849 , read -51247849 ; $J_{0}(2.49,2.50)$, for -49832498 , read -49832489 ; $J_{1}(1.05,1.06)$, for 40692306 , read $30692306 ; J_{1}(1.83,1.84)$, for 243754 , read 253754. $\delta^{2}, J_{0}(.31, .33)$, for -480935 , read -480933 , and for -479731 , read -479733 ; $J_{0}(.47, .48)$, for -467483 , read $-457483 ; J_{0}(.80, .81)$, for -381477 , read -382477 ; $J_{0}(1.05,1.07)$, for -305236 , read -305234 , and for -301835 , read -301837 ; $J_{0}(2.20,2.21)$, for 146183 , read $146103 . \delta^{2}, J_{1}(.13, .14)$, for -52656 , read -52356 ; $J_{1}(1.91,1.92)$, for -406332 , read -406328 .

W. G. BICKLEY, see BAASMTC 5, 9

W. G. BICKLEY \& J. NAYLER

1. Phil. Mag., s. 7, v. 20, 1935, p. 343-347. [X].

M. A. Biot

1. A.S.M.E., Trans., J. Applied Mech., v. 59, 1937, p. A7. [X].

\section{F. F. P. BISACRE}

1. Phil. Mag., s. 6, v. 45, 1923, p. 1035-1038, graph, p. 1036. [XI]. Computation based on Aldis 2, p. 42-43, and JAHNKE \& EMDE 11, p. 139-140, correcting errors in the latter.

E. BJörkdal, see T. Hesselberg

M. BlackMAN

1. R. So. London, Proc., v. 173A, 1939, graph, p. 76. [X].

G. Blanch, see Dinnik 12, Lowan 
H. Blumer (see also Kleinert)

1. Ms. Riccati-Bessel functions $S_{n}(x), S_{n}{ }^{\prime}(x), n=0(1) 7$, for $x=2.5$; $7 \mathrm{D}$, in the Institute for Applied Mathematics, Univ. Berlin (R. v. MISES, Verzeichnis berechneter Funktionentafeln, Berlin, 1928, p. 14). [IV].

2. Z. f. Physik, v. 38, 1926, p. 308. [IV].

3. Z. f. Physik, v. 38, 1926, p. 926-928. [I].

F. BORGNIS

1. Z. f. Physik, v. 100, 1936, graphs, p. 492, 496-498. [V].

M. BORN

1. Optik ein Lehrbuch der elektromagnetischen Lichttheorie, Berlin, Springer, 1933, p. 207-209, 492. [I, III].

M. BORN \& K. FUCHS

1. R. So. Edinburgh, Proc., v. 60, 1940, p. 145. [I].

J. Bouman \& W. F. DE JonG

1. K. Akad. v. Wetens., Afd. Natuurk., Verhandelingen, v. 14, no. 4, 1931, p. 35. [X]. This title was suggested by the Liverpool Index.

J. BOURGET

1. Paris, École Normale Supérieure, Annales, s. 1, v. 3, 1866, p. 82-87. [I]. One of the two tables here given is reprinted in the following entries:

12. RAYLEIGH, Theory of Sound, v. 1, London, 1877, p. 274 (German ed., 1879, p. 364); second ed. 1894 (various reprints) p. 330.

18. BYERLY 1, 1893, p. 286.

14. Thомson, 1893, p. 353.

1. DALE, 1903, p. 83.

16. JAHNKE \& EMDE $1_{1}, 1909$, p. 147; 12, p. 239; 1,-16, p. 168.

17. ReINSTEIN, 1911, p. 75; partial.

18. Gray \& Mathews $1_{2}, 1922$, p. 302.

19. Prescott, 1924, p. 653.

110. HAYAShi 2,1930 , p. 52; partial.

$1_{11}$. Colwell \& Hardy, 1937, p. 1046.

112. F. Bowman, Introduction to Bessel Functions, London, 1938, p. x, partial.

Errata: Of the 54 entries of the Bourget table of roots of $J_{p}(x)=0,27$ are erroneous;

E. BRASEY see $M T A C$, p. 160 . These 27 errors have been corrected in Jahnke \& Emde $1_{6}$.

1. L'Enseignement Math., v. 23, 1924, p. 193-202. [XI].

P. Brauer \& E. Brauer

1. Z. angew. Math., v. 21, 1941, p. 177-182. [IXi].

G. BReIt (see also Yost)

1. Phys. Rev., s. 2, v. 32, 1928, p. 275. [V].

B.A.A.S., Committee for the Calculation of Mathematical Tables (BAASMTC).

Apart from the partially anonymous tables listed below, there are numerous other tables listed elsewhere in this Bibliography under the names of members of this Committee.

1. Bessel Functions. Part I. Functions of Orders Zero and Unity. (Mathematical Tables, v. 6), Cambridge, 1937, xx, 288 p. $21.5 \times 28 \mathrm{~cm}$. [I-III].

Tables: T. I: p. $2 \mathrm{f}, J_{0}(x)$ and $J_{1}(x)$, (the 10 -figure values of this table up to $x=15.5$ were formed by subtabulation to tenths of the original 12-figure values of MEISSEL 1; for the range $x=15.5$ to 25 , the values were taken from H. T. Davis); T. II: p. 171f, Zeros of $J_{0}(x)$ and $J_{1}(x)$, with corresponding values of $J_{1}(x)$ and $J_{0}(x)$, 150 values of each to $10 \mathrm{D}$, (see Davis \& Kirkham); T. IIIA: p. 175, Auxiliary Functions for $Y_{0}(x)$ and $Y_{1}(x),\left[C_{0}(x), C_{1}(x), D_{0}(x), D_{1}(x)\right]$; T. III : p. 176f, $Y_{0}(x)$ and $Y_{1}(x)$, (partially based on Aldis 1 and HAYASHI 3); T. IV: p. 201, Zeros of $Y_{0}(x)$ and $Y_{1}(x)$, with corresponding values of $Y_{1}(x)$ and $Y_{0}(x) ;$ T. V: p. 203f, Auxiliary Functions for $J_{0}(x), J_{1}(x), Y_{0}(x)$ and $Y_{1}(x),\left[\left(A_{0}(x), A_{1}(x), B_{0}(x), B_{1}(x)\right.\right.$; T. VI : p. 214f, $I_{0}(x)$ and $I_{1}(x)$, (based on ALDIS 1); T. VIIA: p. 265, Auxiliary Functions for $K_{0}(x)$ and $K_{1}(x),\left[E_{0}(x), E_{1}(x), F_{0}(x), F_{1}(x)\right]$; T. VII: p. 266f, $K_{0}(x)$ and 
$K_{1}(x)$; T. VIII : p. 272f, $e^{-x} I_{0}(x), e^{-x} I_{1}(x), e^{x} K_{0}(x), e^{x} K_{1}(x) ;$ T. IX: p. $284, e^{x}$ and $e^{-x} ;$ T. XA: p. 285 , Everett coefficients of the second difference; T. XB, XC: Bessellian coefficient of the double second difference. In each T. I-VIII (except in II and IV) second differences are given, modified if necessary, adequate for interpolation within a unit of the last figure given. Prepared under the supervision of L. J. Comrie.

Erratum: missing decimal point in the value of $J_{1}(7.840), \mathrm{G}$. R. Clements, Amer. Math. So., Bull., v. 44, 1938, p. 767. The names Bôcher and Schafheitlin are twice misspelled on p. xix, xx. See also Meissel 2 .

2. Mss. $-J_{n}(x), x=[0(.1) 25 ; 8 \mathrm{D}], n=2(1) 20$, expanded for $x=[0(.01) 10 ; 8 \mathrm{D}]$, $n=2(1) 12$, and with some other minor variations; second differences are given, modified if necessary. Also $J_{n}(x)$, for $x=[0(.1) 25 ; 10 \mathrm{D}], n=0(1) 20$, without differences. (Copies of these mss. are also in possession of the NYMTP). Prepared under the supervision of L. J. Comrie. [I]. See Comrie.

3. Mss. $-Y_{n}(x)$, or in some ranges $y_{n}(x)=x^{n} Y_{n}(x), x=[0(.1) 25 ; 8 \mathrm{~S}], n=2(1) 12$; second differences, modified if necessary. Also $Y_{n}(x)$, for $x=[.1(.1) 25 ; 10 \mathrm{~S}]$, $n=0(1) 20$, without differences. (A copy of this latter ms. is in the possession of the NYMTP). Prepared under the supervision of J. C. P. MiLler (the values from $x=20$ to $x=25$ were made available by C. E. GwYTHER); not quite complete. [II].

4. Mss. $-I_{n}(x)$, and $i_{n}(x)=x^{-n} I_{n}(x)$, and $e^{-x} I_{n}(x), x=[0(.1) 20 ; 8 \mathrm{~S}], n=2(1) 20$, expanded for $x=[0(.01) 10 ; 8 \mathrm{D}], n=2(1) 12$; second differences, modified if necessary. Also $I_{n}(x)$, for $x=[0(.1) 20 ; 10 S], n=0(1) 20$, without differences. (A copy of this last ms. is in the possession of the NYMTP). Prepared under the supervision of J. C. P. Miller and A. J. Thompson; not quite complete. [III].

5. Mss. $-K_{n}(x)$, and $k_{n}(x)=x^{n} K_{n}(x)$, and $e^{x} K_{n}(x) ; x=[.1(.1) 20 ; 8 \mathrm{~S}], n=2(1) 20$; expanded for $x=[0(.01) 10 ; 8 \mathrm{D}], n=2(1) 12$; second differences, modified if necessary. Also $K_{n}(x)$, for $x=[0(.1) 20 ; 10 \mathrm{~S}], n=0(1) 20$, without differences. Prepared under the supervision of W. G. BICKLEY; not quite complete. [III].

6. Mss.-Preliminary tables for Nos. 2-5:

$J_{n}(x), x=[0(.1) 25.5 ; 12 \mathrm{D}], n=0(1) 20$.

$Y_{n}(x), x=[.1(.1) 25.5 ; 13-20 \mathrm{D}], n=0(1) 21 ; y_{n}(x), x=[0(.1) 6 ; 14 \mathrm{D}], n=0(1) 20$, with some extension to higher $x$ for the higher values of $n$.

$I_{n}(x), x=[0(.1) 20 ; 15-18 \mathrm{D}], n=0(1) 21 ; \log I_{n}(x), x=[6(.1) 20 ; 15 \mathrm{D}], n=20$, $21 ; i_{n}(x), x=[0(.1) 6 ; 15 \mathrm{D}], n=0(1) 22 ; \log i_{n}(x), x=[6(.1) 20 ; 15 \mathrm{D}]$, $n=21,22$.

$K_{n}(x), x=[.1(.1) 20.5 ; 12-13 \mathrm{~S}], n=0(1) 20 ; k_{n}(x), x=[.1(.1) 6 ; 12-15 \mathrm{~S}], n=0(1) 20$. Most of the above tables have been checked to 12 or more decimals by formation of differences to high orders or otherwise; differences are also available in manuscript. [I-III].

7. Mss. $-J_{1 / 4}(x), J_{-1 / 4}(x), J_{3 / 4}(x), J_{-3 / 4}(x), x=[0(.1) 20 ; 7 D]$. [VI]. Subtabulation in the range $0<x<5$, reported in 1933, appears not to have been carried out.

8. A. Printers proof, and $B$. Mss. of Airy's Integral, see Section V of this Guide. Contents of $A$, of which J. C. P. MILlER is the chief author, and which the BAASMTC hopes to publish in 1944: T. I: $A i(x)$ and $A i^{\prime}(x), x=[-20(.01)+2 ; 8 \mathrm{D}]$; T. II : Log $A i(x)$ and $A i^{\prime}(x) / A i(x), x=0(.1) 25(1) 75,8 D$ and 7D; T. III : Zeros and turning values of $A i(x)$ and of $A i^{\prime}(x)$, the first 50 of each, to 8D; T. IV: $B i(x)$ and reduced derivatives, $x=[-10(.1)+2.5 ; 8,9$ or $10 \mathrm{D}] ; \mathrm{T} . \mathrm{V}:$ Zeros and turning values of $B i(x)$ and of $B i^{\prime}(x)$, the first 20 of each, to $8 \mathrm{D} ; \mathrm{T}$. VI : $\log B i(x)$ and $B i^{\prime}(x) / B i(x)$, $x=[0(.1) 10 ; 8 \mathrm{D}$ and 7D]; T. VII: $F(x), x(x), G(x), \psi(x), x=-80(1)-30(.1)$ $+2.5 ;[F(x)$ and $G(x), 8 \mathrm{D} x \leq 0 ; 7 \mathrm{D} x>0] ; \chi(x)$ and $\psi(x), 6 \mathrm{D}$ of $1^{\circ}$. The reduced derivatives (which are $h^{n}(d / d x)^{n} B i(x) / n$ !, with $h=.1$, the interval of tabulation) are given as far as needed for interpolation. Modified second differences, with a fourth difference correction when needed are provided for interpolation in T. I, II, VI, and VII. [V]. A 7-place table $A i(x)$ by HAROLD JefFreYs was incorporated into this material. [See H. Jefrereys, Phil. Mag., s. 7, v. 33, 1942, p. 451-456.] 
$B$. Mss.-All the tables listed above to two or more extra decimals. In addition the following are available: $(a) A i(x)$ and reduced derivatives as far as needed for step-by-step integration to 12 working decimals for $x=-20(.05)-10(.1) 0$; and to 12 working $S(21 \mathrm{D}$ at $x=10)$, for $x=0(.1) 10$. (b) $B i(x)$ and reduced derivatives, as far as needed for step-by-step integration to 12 working decimals for $x=-10(.1)$ +2.8. (c) Various squares and derivatives, etc. of the auxiliary functions. [V]. Great help with the preparation of some of the fundamental tables was received from C. E. Gwyther, and the troublesome subtabulations were carried out by H. O. Hartley and Mrs. R. O. Cashen. A. N. Lowan reported that the NYMTP possesses mss. sent to it by J. C. P. MiLler; these are 12-place tables of $A i(x)$, $x=-10(.1)+10$; and tables $(b)$ above.

9. Mss. prepared under the supervision of W. G. Bickley and J. C. P. Miller, These tables contain the first 52 or 53 positive zeros of $J_{n}(x)$ and $Y_{n}(x)$, for $n=\left[0\left(\frac{1}{2}\right) 11(1) 20 ; 12 \mathrm{D}\right]$. [I, II, IV]. The computation is almost complete except in those cases where the McMahon asymptotic expansion fails to give a root to the requisite number of decimals with 8 or 9 terms, and which also have this root exceeding $x=25$ for $J_{n}(x)$ or $x=21.6$ for $Y_{n}(x)$. These exceptions are at present available only to a lesser number of decimals, from about 2 , when $x$ just exceeds 25 or 21.6 , to 10 or 11 .

The calculations have been carried out as follows: by C. E. GwYTHER and others, for $n=3 \frac{1}{2}\left(\frac{1}{2}\right) 11(1) 20$; by S. Johnston for $n=2$ and 3,8 th to 52 nd or 53 rd zeros, also all zeros of $J_{n}(x)$ for which $x<25$; by C. W. Jones and others for zeros of $Y_{n}(x)$ with $x<21.6$.

E. BRIXY

1. Z. angew. Math. Mech., v. 19, 1939, p. 372-379. [I].

2. Z. angew. Math. Mech., v. 20, 1940, p. 236-238. [X].

H. BuchHOLZ

1. Archiv f. Elektrotechnik, v. 22, 1929, p. 367, graphs p. 367, 372. [XI].

2. Elek. Nach. Tech., v. 15, 1938, p. 310, graph, p. 302. [III].

3. Archiv f. Elektrotechnik, v. 37, 1943, p. 148-149, 160-161. [I, IV].

E. BuCHWALD

1. Annalen $d$. Physik, s. 4 , v. 66,1921, p. 5, 12-13 graph p. 4. [I, III].

C. R. BURCH \& N. R. DAvis

1. Phil. Mag., s. 7, v. 1, 1926, graph p. 781. [XI].

V. R. BURSIAN

1. Leningrad, Universitet, Uchenye Zapiski, seriıa fizicheskikh nauk, v. 1, 1935, p. 1-9. [V].

V. R. Bursian \& V. A. FoK

1. Akad. Nauk., Leningrad, Fiziko-Mat. Institut, Trudy (Travaux), v. 2, no. 1, 1931, p. 6-10. $[\mathrm{X}]$.

\section{S. BUTTERWORTH}

1. Phys. So., Proc., v. 25, 1913, p. 297. [I, XI].

2. R. So. London, Trans., v. 222A, 1921, p. 64-65, 73, graphs, p. 68,71 . [XI].

A. Buxton

1. R. A. S., Monthly Notices, v. 81,1921, p. 558. [I]. This title was suggested by the Liverpool Index.

W. E. Byerly (see also Bourget, P. A. Hansen, Stokes)

1. An Elementary Treatise on Fourier's Series and Spherical, Cylindrical and Ellipsoidal Harmonics . ..., Boston, 1893, p. 286-287. [I].

Tables: T. IV: $x / \pi$ for $J_{0}(x)=0$ and $J_{1}(x)=0$; T. V: Zeros of $J_{n}(x)$; T. VI: $J_{0}(x), J_{1}(x)$.

2. Harmonic Functions, in Higher Mathematics ed. M. MERriman and R. S. WoodWARD, New York, 1896, p. 224-225. [I, III]. 
2. Second ed., 1898, third ed., 1900. Also as a separate book, fourth ed. enl. 1906, p. 62-63.

Contents: T. 2: $J_{0}(x), J_{1}(x)$; T. $3: x_{8}, x_{8}{ }^{\prime} ;$ T. $4: J_{0}(x i)=I_{0}(x)$.

G. A. Campbell \& R. M. Foster

1. Bell Telephone System, Technical Publications, Monograph B584, 1931, graph p. 20. [VI].

12. Collected Papers of George Ashley Campbell, New York, Amer. Tel. and Tel. Co., 1937, graph, p. 308.

H. Carrington

1. Phil. Mag., s. 6, v. 50, 1925, p. 1262. [III].

Erratum: First root for $n=0$, for 3.1961, read 3.1955. AIREy 2, p. 227 (MTAC, p. 202).

Mrs. R. O. CASHEN, see BAASMTC 8.

A. Cayley, see Rayleigh

S. Chandrasekhar

1. R. A. S., Mo. Notices, v. 94,1933 , p. 27,34 . [III, X].

S. Chapman \& T. T. Whitehead

1. Int. Congress Mathematicians, Toronto, 1924, Proc., Toronto, v. 2, 1928, p. 329330. [IV].

C. Chree

1. Cambridge Phil. So., Trans., v. 15, 1891, p. 221-223, 227, 229, 230. [I].

A. H. Church, see Newman

M. M. ClaPP

1. J. Math. Phys., M.I.T., v. 16, 1937, p. 76. [I].

M. Clarke, see Jaeger

G. R. Clements, see BAASMTC 1.

R. C. Colwell \& H. C. Hardy (see also Bourget)

1. Phil. Mag., s. 7, v. 24, 1937, p. 1044, 1046-1049, 1053-1054 and graphs, p. 10471049. [I, II, III].

Errata: "It is quite unhappy for us to mention here that some misprints and few numerical values too approximated have slipped into the excellent table," p. 1044, Osaka and ToBIISI, p. 777.

In the table of $I_{0}(x), I_{0}(30)$, p. 1046 , is erroneous; see $M T A C$ p. 199-200, note by J. W. Wrench, JR., who gives $I_{0}(30)$ to $20 \mathrm{~S}$. See also Bourget.

R. C. Colwell, J. K. Stewart, \& H. D. Arnett

1. Acoustical So. Amer., J., v. 12, 1940, graph p. 262. [III].

L. J. Comrie (see also Airey 34, BAASMTC 1, 2, 6, McLachlan \& Meyers)

1. Ms. $-J_{0}(x)$ and $J_{1}(x)$, for $x=[0(.001) 16(.01) 25 ; 12 \mathrm{D}]$. [I]. See $M T A C$, p. 60-61.

E. U. CoNDoN

1. J. Applied Physics, v. 11, 1940, p. 506. [I].

A. E. ConRady

1. R.A.S., Mo. Notices, v. 79, 1919, p. 577. [I]. This title was suggested by the Liverpool Index.

M. S. Corrington \& W. Miehle

1. Mss. in possession of the RCA Victor Division of Radio Corporation of America, Camden, N. J., $J_{n}(m s)$ for $n=0(1) 10, s=1(1) 20$, (a) $m=0(1) 10$; (b) $m=\pi(\pi) 5 \pi$, each table being to $5 \mathrm{D}$. [I].

O. G. C. DAHL

1. A.S.M.E., Trans., v. 46, 1924, graphs, p. 170-171. [I, II].

J. B. Dale (see also Bourget, Lodge, LoMmel, StoKes)

1. Five-Figure Tables of Mathematical Functions comprising tables of logarithms, powers of numbers, trigonometric, elliptic, and other transcendental functions, London, 1903, p. 81; Table probably adapted from MeIsSEL 4. [I, III].

Erratum: P. 80, $J_{0}(5.9)$, for +.11203 , read +.12203 . Liverpool Index. 
Darmstadt, Technische Hochschule

1. Ms. seven-place table of the function $\Lambda_{n}(x)$, JAHNKE \& EMDE $1_{2}$, p. 221. [VII].

F. N. David \& E. C. Fieller

1. Biometrika, v. 24,1932 , p. $344-346$. [X].

A. H. Davis

1. Modern Acoustics, New York, Macmillan, 1934, graph, p. 62. [I, IX].

H. T. Davis

1. Mss. $-J_{0}(x)$ and $J_{1}(x)$ for $x=$ [15.5(.01)25; mostly $15 \mathrm{D}$ ]. [1]. The calculation was based on MEISSEL 5; see BAASMTC 1, p. xi.

H. T. Davis \& W. J. Kirkham (see also BAASMTC 1)

1. Am. Math. So., Bull., v. 33, 1927, p. 760-772. [I].

Errata: In the value given for $J_{1}\left(x_{4}\right)$ for -0.2324598214 , read -0.2324598314 . Meissel's correct value for $x_{8}^{\prime}$, in MeIsSEL 2, was copied incorrectly in both editions of GraY \& MATHEWs; for 29.0468285340 , read 29.04682 85349. There are the following 11 cases with incorrect end-figures in $J_{1}\left(x_{8}\right)$; when $s=7,2$ should be substituted for $3 ; s=8,7$ for $4 ; s=9,8$ for $9 ; s=10,7$ for $9 ; s=11,4$ for $5 ; s=14$, 8 for $9 ; s=17,0$ for $2 ; s=18,3$ for $4 ; s=20,89$ for $91 ; s=24,8$ for $9 ; s=27$, 3 for 4 . Four other cases of end-figure corrections are as follows: in $x_{s}^{\prime}, s=53$, 7 for $8 ; s=54,2$ for $1 ; s=55,0$ for 1 ; and in $J_{0}\left(x_{s}{ }^{\prime}\right), s=150,5$ for 6 . (BAASMTC 1, p. xiii).

12. T. II of BAASMTC 1 , is a corrected reprint of the first 150 zeros, $x_{s}, x_{s}^{\prime}$, of $J_{0}(x)$ and $J_{1}(x)$, together with the values of $J_{0}\left(x_{s}{ }^{\prime}\right), J_{1}\left(x_{s}\right)$.

N. R. DAvis, see BURCH

P. Debye

1. Annalen d. Physik, s. 4, v. 46, 1915, p. 820. [X].

G. Della Moglie

1. Accad. d. Lincei, Rendiconti, cl. sci. fis. mat., s. 6, v. 11, 1930, p. 955, 956, 959, graph, p. 957. [II].

J. E. Dickmann

1. Ingenieur Archiv, v. 9, 1938, p. 478. [III].

A. N. Dinnik (see also Hayashi)

1. Russ. fiziko-khimicheskoe Obshchestvo, Zhurnal, v. 42, 1911, part 8, 4 p. [V].

2. Arch. Math. Phys., s. 3, v. 18, 1911, p. 337-338. [V].

Errata: Greenhill's erroneous $x_{1}$ in $J_{-1 / 3}(x)$ copied.

3. Novocherkask, Donskoǐ Politekh. Institut, Izvestizâ, v. 1, 1912, p. 30-31. [V].

4. Novocherkask, Donskoì Politekh. Institut, Izvestîa, v. 1, 1912, p. 326-328. [IV].

5. Novocherkask, Donskoì Politekh. Institut, Izvestiza, v. 2, 1913, p. 57-58, 68. [V, VI].

6. Novocherkask, Donskoì Politekh. Institut, Izvestiza, v. 2, 1913, p. 353-356. [IV, $\mathrm{V}, \mathrm{VI}]$.

7. Arch. Math. Phys., s. 3, v. 20, 1913, p. 238-240. [IV].

8. Arch. Math. Phys., s. 3, v. 21, 1913, p. 325-326. [VI].

8. JAHNKE \& EMDE, $1_{5}-1_{5}$, p. 164 , and $1_{2}$, p. 223.

Errata: In reprinting these tables of $J_{ \pm 1 / 4}(x), J_{ \pm 8 / 4}(x)$, EMDE made 10 corrections, namely in: $J_{1 / 4}(4.8), J_{1 / 4}(5.4), J_{3 / 4}(1), J_{3 / 4}(4.8), J_{3 / 4}(5.4), J_{-1 / 4}(4.8), J_{-1 / 4}(5.4)$, $J_{-3 / 4}(4.8), J_{-3 / 4}(5.4), J_{-3 / 4}(5.6)$, and one change from what was right $J_{3 / 4}(5.2)$. But after these changes the NYMTP found 31 errors, the following 9 of which were larger than a unit in the last figure: $J_{1 / 4}(7.4)$, for .2925 , read $.2923 ; J_{1 / 4}(7.6)$, for .2873, read .2869; $J_{1 / 4}(7.8)$, for .2711 , read $.2704 ; J_{1 / 4}(8.0)$, for .2449 , read $.2436 ; J_{3 / 4}(6.8)$, for .0447 , read $.0449 ; J_{3 / 4}(7.8)$, for .2602 , read $.2604 ; J_{3 / 4}(8.0)$, for .2749 , read $.2752 ; J_{-1 / 4}(.2)$, for 1.4310 , read $1.4319 ; J_{-3 / 4}(3.2)$, for -.3943 , read - 3945. All of the errors of this table have been corrected in JAHNKE \& EMDE $1_{6}$.

9. Arch. Math. Phys., s. 3, v. 22, 1914, p. 227. [V]. 
9. JAHNKE \& EMDE, $1_{8} 1_{5}$, p. 235, and 12, p. 285.

Errata: The following errors were noted by F. P. White, R. So. London, Proc., v. $100 \mathrm{~A}, 1922$, p. 522 ; in $I_{-1 / 3}(x), x=2.2$, for 2.5226 , read $2.5626 ; x=3.00$, for 4.9710, read 4.7754; $x=6$, for 65.55 , read 66.55 ; in $I_{-2 / 3}(x), x=2.8$, for 3.7002 , read 3.7595; $x=8.0$, for 418.01 , read 415.01 . As the result of inquiries carried through by the NYMTP we are able to report that in these tables of $I_{ \pm 1 / 3}(x), I_{ \pm 2 / 3}(x)$ there are 63 errors in the 168 entries; 26 of these are greater than unity in the last figure and some of the errors are serious. It was shown that White's corrected value, noted above, for $I_{-2 / 3}(2.8)$ is incorrect; for 3.7595, read 3.7603. This correct value was given in Dinnik 15. See also Dinnik 11, T. IV, the unreliability of which was voiced by Heatley 1, p. 274. The 63 errors have been corrected in JAHNkE \& EMDE 16.

10. Prilozhenie Funktsiü Besselia $k$ zadacham Teorii uprugosti [Application of Bessel functions to problems in the theory of elasticity], part 2, Teoriia vibratsiu, Dnepropetrovskiǔ Gornyĭ Institut [School of Mines], Izvestîa, v. 11, no. 2, 1915, p. 19-20, 23-24, 130-135. [II, V, VI].

Tables: T. I: $J_{ \pm 1 / 4}(x), J_{ \pm 8 / 4}(x)$; T. II : zeros of $J_{ \pm 1 / 4}(x), J_{ \pm 3 / 4}(x)$; T. III : $I_{ \pm 1 / 4}(x)$, $I_{ \pm 8 / 4}(x) ;$ T. IV: $I_{ \pm 1 / 3}(x), I_{ \pm 2 / 3}(x)$.

Errata: All the errors noted in connection with DinNik 8 and 9, are also preserved in T. I and IV.

11. All Russian Central Committee, [Communications], 1922, p. 121-126. [III, XI].

11. Hayashi 2, p. 105-109.

Errata: Hayashi's reprint of Dinnik's table preserves all its mistakes; 53 errors of more than a unit in the final decimal place have been listed by A. N. LowAN and G. BLANCH, "Errors in Hayashi's [sic] table of Bessel functions for complex arguments," Amer. Math. So., Bull., v. 47, 1941, p. 291-293.

12. A.S.M.E., Trans., v. 51 (1), 1929, APM-51-11, p. 109. [II].

13. A.S.M.E., Trans., v. 54, 1932, APM-54-16, p. 170-171. [II].

14. Tablitsi Besselevikh Funktsii drobovogo porâadku [Tables of Bessel functions of fractional order], Akad. Nauk, U.S.R.R., Kiev, Prirodnicho-tekhnichiniı̌ vidil, 1933, 29 p. [IV-VI].

Tables: T. I : $J_{ \pm 1 / 2}(x), I_{ \pm 1 / 2}(x)$; T. II : $J_{ \pm 1 / 3}(x), J_{ \pm 2 / 3}(x)$; T. III : zeros of $J_{-2 / 3}(x)$, $J_{-1 / 2}(x), J_{1 / 3}(x), J_{2 / 3}(x)$; T. IV: $I_{ \pm 1 / 3}(x), I_{ \pm 2 / 3}(x)$; T. V: $J_{ \pm 1 / 4}(x), J_{ \pm 3 / 4}(x)$; T. VI : zeros of $J_{ \pm 1 / 4}(x), J_{ \pm 3 / 4}(x)$; T. VII : $I_{ \pm 1 / 4}(x), I_{ \pm 3 / 4}(x)$; T. VIII : $J_{ \pm 1 / 6}(x), J_{ \pm 5 / 6}(x)$; T. IX: zeros of $J_{ \pm 1 / 6}(x), J_{ \pm 5 / 6}(x)$; T. X: $I_{ \pm 1 / 6}(x), I_{ \pm 5 / 6}(x)$; T. XI-XII $: J_{n}(x), I_{n}(x)$ for various values of $n$ (mostly fractional), and of $x$.

Errata: We have a report prepared by the NYMTP, after checking with its mss., Dinnik's T. II, III, V-VII, and T. IV, up to $x=12$. Apart from innumerable endfigure unit errors, there are 323 errors of more than one unit; four samples are as follows: $J_{1 / 3}(6.1)$, for -.0217 , read $+.0217 ; J_{2 / 3}(1.8)$, for .5978 , read $.6016 ; I_{1 / 3}(12)$, for 18757 , read $18857 ; J_{+1 / 4}(.4)$, for .4173 , read .7144 . The corrections for 8 zeros of T. III and T. VI are as follows: fif th zero, $J_{2 / 8}$, for 15.970 , read 15.964 ; first zero, $J_{-1 / 8}$, for 1.860 , read 1.866 ; fourth zero, $J_{-1 / 3}$, for 11.263 , read 11.237 ; fif th zero, $J_{-1 / 3}$, for 14.406 , read 14.404 ; fourth zero, $J_{-1 / 4}$, for 11.404 , read 11.396 ; first zero, $J_{8 / 4}$, for 3.441 , read 3.491 ; fif th zero, $J_{3 / 4}$, for 16.110 , read 16.091 ; fourth zero, $J_{-3 / 4}$, for 10.590 , read 10.588 .

Nos. 1, 10, 11, 14 are in the Library of Brown University and nos. 3-6 in the New York Public Library.

A. T. Doodson (see also Proudman)

1. B.A.A.S., Report, 1914, p. 87-102. [IV].

Errata: Errors in $\left|E_{13}(9)\right|^{2}$ and $\left|E_{14}(9)\right|^{2}$, and in characteristics of various logarithms are corrected in Doodson 2, p. 97.

2. B.A.A.S., Report, 1916, p. 97-107. [IV].

3. B.A.A.S., Report, 1922, p. 263-270. [IV]. 
F. Dubois

1. Über die Festigkeit der Kegelschale, Diss. Eidg. Techn. Hochschule, Zürich, 1917, p. 126-127, Kurvenblatt 1-3. [XI]. (See F. EmDE, Z. angew. Math. Mech., v. 20, 1940, p. 128.) There is a copy of this dissertation in the M.I.T. Library; a film copy is in the Brown Univ. Library.

H. B. DwIGHT

1. A.I.E.E., $J$., v. 42,1923 , p. 830 . This table of ber $x$, bei $x$, ber' $x$, bei' $x$, $\operatorname{ker} x$, kei $x$, $\operatorname{ker}^{\prime} x, \operatorname{kei}^{\prime} x$, is compiled from A. G. Webster, SAvidge 2 and 3. Error in bei' 3.7, see A. G. Webster 1. [XI].

12. No. $3_{1}, 1929$, T. I, p. 813.

13. Table of Integrals and other Mathematical Data, New York, 1934, T. 1050, p. 211-217, case when $n=0$.

Tables: $\operatorname{ber}_{n} x, \operatorname{bei}_{n} x, \operatorname{ber}_{n}^{\prime} x, \operatorname{bei}_{n} x, \operatorname{ker}_{n} x, \operatorname{kei}_{n} x, \operatorname{ker}_{n}{ }_{n} x, \operatorname{kei}_{n}{ }_{n} x, n=0(1) 5$.

2. A.I.E.E., Trans., v. 42, 1923, p. 858. [XI].

2. No. $3_{1}, 1929$, T. II, p. $813-814$.

2 . No. $1_{3}, 1934$, p. 211-216, practically all of the table.

$3_{1}$. A.I.E.E., Trans., v. 48, 1929, T. I-III, p. 813-815. [XI].

3. No. $1_{3}$, p. $216-217$.

4. A.I.E.E., Trans., v. 58, 1939, p. 788-790; computations by students at M.I.T. The first half of T. I is in A. G. WEBSTER, and parts of T. II-III are in ALGER 1. [XI].

5. A.I.E.E., Trans., v. 60,1941 , p. 136; computing done by students at M.I.T., based on LODGE 1. [III].

6. Mathematical Tables .... New York, McGraw-Hill, 1941; third impression with additions [1943], p. 214-221. [XI].

Tables: ber $x$, bei $x$, $\operatorname{ber}^{\prime} x$, bei' $x$, and the same in polar form $M, \theta$.

A. S. EDDINGTON

1. R.A.S., Mo. Notices, v. 68,1908 , p. 592. [III, IV]. This title was suggested by the Liverpool Index.

12. SMarT, p. 127.

E. M. ELDERTON

1. Biometrika, v. 21, 1930, p. 195-201. [III, IV].

12. Tables for Statisticians and Biometricians, ed. by K. Pearson, part 2, London, 1931, p. 138-144.

W. M. ELSASSER

1. Heat Transfer by Infrared Radiation in the Atmosphere, (Harvard Meteorological Studies, no. 6), Cambridge, Mass., 1942, p. 30-31. [III].

F. EMde (see also JaHNKe)

1. Archiv Math. Physik, s. 3, v. 24, 1915, p. 249. [V, VI].

2. o Tafeln elementarer Funktionen, Leipzig, Teubner, 1940, xii, 181 p.; see Fort. Math., v. 66,1942 , p. 570 . [IV].

F. EMdE \& R. RÜHLE

1. Deutsch. Math. Ver., Jahresb., v. 43, 1934, p. 269. [XI].

A. ERDÉLYI

1. Annalen d. Physik, s. 5, v. 23, 1935, p. 39. [XII].

H. ERTEL \& J. J.AW

1. Meteorologische $Z$., v. 55, 1938, figures p. 205-206. [III].

L. EULER

1. Introductio in Analysin Infinitorum, v. 2, Lausanne, 1769, p. 318-320. Later print 1797; French translation 1797 and 1835; and German translation 1788 and 1836. [IV].

G. EURINGER

1. Z. f. Physik, v. 96, 1935, graph p. 47. [I].

G. S. FIELD

1. Canadian J. Research, v. 17A, 1939, p. 143-144. [II]

E. C. Fieller, see David 
F. A. Fischer

1. Elek. Nach. Technik, v. 9, 1932, graph, p. 151. [X].

J. Fischer

1. Annalen d. Physik, s. 4, v. 72, 1923, graphs, p. 389, 393. [VI]. Abbreviated diss. Munich. Fischer tells us (p. 389) that in this diss. are tables of $F(x), G(x)$, and also, apparently of $K(x), L(x)$. Jahresverz. d. an d. deutschen Universitäten u. Hochschulen ersch. Schriften, 1922, lists this as only in "Maschinenschrift" form.

2. Ingenieur Archiv, v. 10, 1939, graphs, p. 111. [II].

W. FISCHER

1. Ms. table of $2 J_{1}(x \sqrt{-i}) / x \sqrt{-i} J_{0}(x \sqrt{-i})=Q+i P,[Q=2 Z / x X, P=2 W / x X$, ] for $x=[0(.5) 6(.2) 10(5) 20 ; 4 \mathrm{D}-5 \mathrm{D}]$, and graphs of $Q$ and $-P$, in the Institute for Appl. Math., Univ. Berlin. The graphs of $Q$ and $-P$ were published in a paper of F. Wever and W. FIScher in o Institut $f$. Eisenforschung, Düsseldorf, Mitteilungen, v. 8 , no. 10, 1926, p. 157. (R. v. MISES, Verzeichnis berechneter Funktionentafeln, part 1, Berlin, 1928, p. 30.) [XI]. See MacleaN and ZenNECK.

J. A. Fleming

1. The Principles of Electric Wave Telegraphy and Telephony, third ed., London, Longmans, 1916, p. 135. [XI]. This table of $\frac{1}{2} x W / V$ is taken from that of MACLEAN.

V. A. For (see also Bursian)

1. Akad. Nauk, Leningrad, Doklady (Comptes Rendus), 1934, v. 1, p. 102. [I].

V. A. FoK \& V. A. Kolpinsky

1. Akad. Nauk, Leningrad, Fizicheskiน Zhurnal, Journal of Physics, v. 3, 1940, graph, p. 130. [I].

A. R. Forsyth

1. Messenger Math., s. 2, v. 50, 1921, p. 135, 139-146, 148. [I, IV].

R. M. Foster, see Campbell

F. E. FowLE

1. Smithsonian Physical Tables, seventh rev. ed., Washington, D. C., 1920, p. 66-68. [I]. Tables: $J_{0}(x), J_{1}(x), x_{8}, x_{8}{ }^{\prime}, J_{0}\left(x_{8}{ }^{\prime}\right)$, based on GRAY \& MATHEws $1_{1}, \mathrm{~T}$. I, and MeISSEL 1 ; these tables do not occur in an earlier edition.

12. Eighth rev. ed. 1932 ; reprint of eighth rev. ed., 1934, p. 68-70. In addition to the above tables is also a table of $J_{1}\left(x_{8}\right)$.

R. H. Fowler, see Milne

F. E. Fox

1. Acoustical So. Am., J., v. 12, 1940, p. 148-149. [XII].

G. Franke

1. Annalen d. Physik, s. 5, v. 2, 1929, p. 652, 653. [III].

K. Franz

1. Elek. Nach. Technik., v. 16, 1939, graphs, p. 25. [IV].

A. J. Fresnel

1. Acad. d. Sc., Paris, Mémoires, v. 5 for 1821-22, 1826, p. 408, 430f. [X, XII]. Table of $S(u), C(u), u=[0(.1) 5.5 ; 4 \mathrm{D}]$. Fresnel distributed this table to $u=5.1$ in 1818 .

12. AIry 1,1831, p. 399 . Table of $S(u), C(u)$, to $u=5.5$, apparently calculated without knowledge of Fresnel's table.

13. Gilbert, 1863 , p. $48, u=[0(.01) .1(.05) 1(.1) 5.5 ; 4 \mathrm{D}]$; thus, while some improvements were made, very little is added to Fresnel's table. This table for $S(u), C(u)$ is reproduced in MAYALL.

14. Fresnel, Oeuvres, v. 1, Paris, 1866, p. $319,341$.

K. Fuchs (see also Born)

1. R. So. Edinburgh, Proc., v. 61, 1941, p. 36. [I].

C. C. Furnas

1. Am. Math. Mo., v. 37, 1930, p. 287 , graph, p. 284. [III]. Last significant figure doubtful. 
R. GANS

1. Annalen d. Physik, s. 4, v. 47, 1915, p. 729, 732. [V].

2. Physik. Z., v. 25, 1924, p. 335. [I, IV].

3. Annalen d. Physik, s. 4, v. 76, 1925, p. 35. [IV].

4. Annalen d. Physik, s. 4, v. 78, 1925, p. 12, 19, 22, 23; graphs, p. 24-25, 28. [I, X]. P. GILBERT (see also FrESNEL)

1. Acad. d. Sci., Brussels, Mémoires, v. 31, 1863, p. 47-51. [X, XII].

S. P. Glazenap

1. Matematicheskie $i$ Astronomicheskie Tablițy [Mathematical and Astronomical Tables], Leningrad, 1932, p. 91-115. [I, II, V, IX, X].

Tables: T. XX 1: $J_{0}(x), Y_{0}(x) ; \mathrm{T} . \mathrm{XX} 1 \mathrm{a}: \frac{1}{2} \int_{0}^{x} J_{0}(t) d t, \frac{1}{2} \int_{0}^{x} Y_{0}(t) d t$, maxima and minima of these integrals; T. XX 2: $J_{1}(x), Y_{1}(x) ; \mathrm{T} . \mathrm{XX} \mathrm{2a:} \frac{1}{2} \int_{0}^{x} J_{ \pm 1 / 2}(t) d t$; T. XX 3: $J_{1 / 3}(x), Y_{1 / 3}(x) ;$ T. XXI: Zeros of $J_{0}(x), J_{1}(x), Y_{0}(x), Y_{1}(x), J_{1 / 8}(x)$, $Y_{1 / 3}(x)$; T. XXII : $H_{0}(x), H_{1}(x)$.

S. GoldsteIN

1. R. So. London, Proc., v. 123A, 1929, p. 446, 449-450, 456. [IXi, IX].

A. R. Gordon see W. L. MiLler

S. GRADSTEIN, see JahNKE \& EMDE 1,

R. GRAMMEL

1. Ingenieur Archiv, v. 6, 1935, p. 262. [II].

2. Ingenieur Archiv, v. 6, 1935, p. 445, graphs, p. 447. [II].

3. Ingenieur Archiv, v. 9, 1938, graph, p. 133. [IX].

A. Gray \& G. B. Mathews

1. A Treatise on Bessel Functions and their Applications to Physics, London, 1895, p. 155, 180,182-183, 207, 245-288. [I, III, XI, XII].

Tables: T. I: $J_{0}(x), J_{1}(x)$, reprint of Meissel 1 ; T. II : $J_{n}(m)$ from an unpublished ms. of E. Meissel published in MeIssel 5; T. III: Zeros of $J_{1}(x)$ with corresponding maximum or minimum values of $J_{0}(x)$, reprinted from MeIsseL 2; T. IV: $J_{0}(x i \xi)$ T. V: $I_{1}(x)$; T. VI $: I_{m}(x)$; T. IV-VI are reprints of parts of LODGE 1-2. The table on p. 155 is from Thomson, on p. 182-183 from Lommel, and on p. 207 from Struve 1.

12. Second ed., by A. Gray \& T. M. MacRobert, London, Macmillan, 1922; reprinted 1936, p. 170, 192, 194-195, 218, 264-317. [I, III, V, VI, XI, XII].

Tables: T. I-II as in the first ed.; T. III : Zeros of $J_{0}(x)$ with corresponding values of $J_{1}(x)$, reprinted from WILLSON and PEIRCE; T. IV: same as T. III of first ed.; T. V: from BourGET; T. VI, VIII, IX are the same as T. IV-VI in the first ed.; T. VII: $I_{0}(x)$ reprint of a portion of LoDGE 3 ; T. X-XI: $K_{0}(x)$ and $K_{1}(x)$ from ALDIS 1; T. XII: from IsHeRwOoD; T. XIII: from AIREY 18. The table on p. 170 is from Thomson on p. 194-195 from Lommel, and on p. 218 is from Struve 1.

Errata.-The following errata are in both editions: 1 . $J_{0}(.62)$, for .90518 , read $.90618 ; 2 . J_{0}(3.07)$, for 2262330 , read $2262230 ; 3 . J_{1}(7.87)$, for .21401, read .21407; 4. $J_{4}(5)$, for 26304 , read $23604 ; 5$. $J_{28}(6)$ for 02496 , read $02495 ; 6 . J_{30}(14)$, for 538 , read $534 ; 7 . J_{31}(16)$, for 49322 , read $94322 ; 8$. Error in copying Meissel's ninth root of $J_{1}(x)=0$, for 4016 , read 4916 ; 9. $I_{1}(4.86)$, for 21.398859282 , read 21.398959282 . The following errata occur in the first edition only: 10 . $J_{0}(1.89)$, for .28663 , read $.28763 ; 11 . J_{0}(5.90)$, for .11203 , read .12203 . The next erratum is only in the second edition: 12 . T. III, $J_{1}\left(x_{0,35}\right)$, for .7635913, read .7636383. (The corrections nos. $1-7$, 10, 11, were given by AIREy 26, p. 297; nos. 8,9 by BAASMTC 1, p. xiii, xvii;

N. GrishKov no. 12 by Davis and Kirkham.) See also Lommel 2.

1. Wissenschaftliche Schriften-[Trudi] des Donnetz Technikums des Genossen Artjem zu Stalin, v. 1, Stalin, 1925, p. 52-57. [V].

F. W. Grover, see Rosa 
C. E. GwYTHER, see BAASMTC 3, 8, 9.

B. HAGUE

1. Phys. So. London, Proc., v. 29, 1917, graphs p. 212-213, and plate oppo. p. 212. [I]. E. HALLEN

1. Arkiv f. Mat., Astr. o. Fysik, v. 24 A, no. 5, 1933, p. 35-36. [II].

2. Archiv f. Elektrotechnik, v. 32, 1938, graphs, p. 515-537. [II].

Hamburg, Sternwarte

1. Sammlung von Hilfstafeln, A-F, Hamburg, 1916, p. A46-A47. [I]. Copy at the Harvard College Observatory.

Tables: $J_{0}(x)$ and $J_{1}(x)$ based on Bessel, P. A. HANSEN (Schlömilch), Meissel 1; W. R. HAMILTON

$x_{8}, J_{1}\left(x_{8}\right)$ based on Willson and PeIRCE; $x_{8}{ }^{\prime}, J_{0}\left(x_{8}{ }^{\prime}\right)$ based on Meissel 2.

\section{HAMY}

1. Phil. Mag., s. 4, v. 14,1857 , p. 381 . [I].

1. Inst. d. France, Acad. Sci., Comptes Rendus, v. 175, 1922, p. 1125. [I, VII].

P. A. HANSEN (see also Hamburg)

11. Schriften der Sternwarte Seeberg, part 1, Gotha, 1843, p. 159-167. [I]. Copy of this publication at Princeton University; film copy at Brown University. T. I is a 6-place abridgment of the 10-place table of BESSEL for the range $0(.01) 3.2$, and extended to 20. See $M T A C$, p. 194.

1. French translation by V. MauvaIs, Connaissance des Temps . . pour l'an 1847, Paris, 1844, p. 159-167. Reprinted as a separate issue, Paris, 1845.

18. O. Schlömilch, Z. Math. Phys., v. 2, 1857, p. 158-165.

14. E. Lommel, Studien über die Bessel'schen Funktionen, Leipzig, 1868, p. 135. There is a 4-place abbreviation of part of T. I in ByERLY 1, and a similar 4-place table in RAyleIGH The Theory of Sound, v. 1, London, 1877, p. 265; second ed., 1894 and W. W. HANSEN reprints of 1926, 1929, 1940, p. 321; German ed., 1879, p. 351-353.

1. J. Appl. Physics, v. 10, 1939, graph p. 44. [VI].

H. C. HaRdy, see Colwell

H. O. HARTLEY, see BAASMTC 8

D. R. HARTREE \& S. JohNSTON

1. Manchester Lit. Phil. So., Memoirs, v. 83, 1939, p. 188. [VI]. This title was suggested by the Liverpool Index.

Eva HaURvitz, see Morse

T. H. HAVELOCK

1. R. So. London, v. 108A, 1925, p. 82. [X].

2. R. So. London, v. 108 A, 1925 , graphs, p. 586,590 . [X].

3. R. So. London, v. 136A, 1932, graphs, p. 469-470. [X].

H. V. Hawkins, H. W. TOBEY \& M. LEE

1. A.S.M.E., Trans., v. 59, 1937, p. A14-A15. [XI]. Table in article by S. C. Hollister.

G. E. HAY

1. Can. J. Research, v. 17A, 1939, p. 135. [X].

K. HAYASHI

1. Tafeln der Besselschen, Theta-, Kugel-, und anderer Funktionen, Berlin, Springer, 1930 , p. $1-47,52-59,119-120.21 .3 \times 27.4 \mathrm{~cm}$. [I, IV].

Tables: T. I: $J_{0}(x), J_{1}(x)$; T. II : $J_{3}(x), J_{-3}(x), J_{3 / 2}(x), J_{-3 / 2}(x)$; T. III and Anhang $J_{n}(x)$.

Errata.- $J_{1}(.69)$, for 1431373 , read $1471373 ; J_{0}(2.81)$, for 6508066 , read 6518066 ; $J_{0}(3.07)$, for 2262330 , read $2262230 ; J_{0}(4.57)$, for 4907335 , read $4907535 ; J_{1}(7.87)$, for 0.21401 , read $0.21407 ; J_{1}(13.31)$, for 1207404 , read 1207414 . (BAASMTC 1 , p. xii.)

2. Fünfstellige Funktionentafeln, Kreis-, zyklometrische, Exponential-, Hyperbel-, Kugel-, Besselsche, elliptische Funktionen, . . ., Berlin, Springer, 1930, p. 81-120. $16.5 \times 24.6 \mathrm{~cm}$. [I-V, X, XI]. 
Tables: T. XV: $J_{0}(x), J_{1}(x), Y_{0}(x), Y_{1}(x)$; T. XVI : zeros of $J_{0}(x)$ and the corresponding values of $J_{1}(x) ; \mathrm{T}$. XVII : zeros of $J_{1}(x)$ and the corresponding values of $J_{0}(x)$; zero of $J_{1000}(x)$ from IkEDA; T. XVIII: zeros of $Y_{0}(x), Y_{1}(x)$; T. XIX: $J_{0}(n \pi)$ from NAGAOKA 1 ; T. XX: $J_{0}\left(r e^{i \theta}\right), J_{1}\left(r e^{i \theta}\right)$, table of DiNNIK 11 reprinted; T. XXI : $Z_{1}(x)$ and $Z_{1}{ }^{\prime}(x)$, tables of SChLEICHER 1 ; T. XXII : $J_{3}(x), J_{-1}(x), J_{3 / 2}(x)$, $J_{-3 / 2}(x)$; T. XXIII-XXIV: $C(u)=\frac{1}{2} \int_{0}^{x} J_{-3}(t) d t, S(u)=\frac{1}{2} \int_{0}^{x} J_{3}(t) d t$, and their maxima and minima; T. XXV: $J_{1 / 8}(x), J_{-1 / 8}(x), Y_{1 / 8}(x), Y_{-1 / 8}(x)$; zero of $J_{-1 / 3}(x)$ from IKEDA.

Errata: For errors in Dinnik's T. XX, see DinNik 11.

3. Tafeln für die Differenzenrechnung sowie für die Hyperbel-, Besselschen, elliptischen und anderen Funktionen, Berlin, Springer, 1933, $21.1 \times 27.6 \mathrm{~cm}$. [I, II].

Tables: T. II: $J_{0}(x), J_{1}(x) \cdots J_{6}(x) ; J_{n}{ }^{\prime}(0), J_{n}{ }^{\prime \prime}(0) \cdots \int_{n}^{(x i v)}(0) ;$ T. V: $Y_{0}(x)$, $Y_{1}(x), J_{0}(x), J_{1}(x) ; \mathrm{T}$. VI : $Y_{0}^{\prime}(x), Y_{0}^{\prime \prime}(x), \cdots, Y_{0}^{x i v}(x) ; \mathrm{T}$. VII $: Y_{n}(16), Y_{n}(17), \cdots$, $Y_{n}(25)$; T. VIII: $J_{0}-J_{2}, J_{2}-J_{4}, \cdots, J_{28}-J_{23}, J_{1}-J_{8}, J_{8}-J_{5}, \cdots, J_{25}-J_{27}$. See BAASMTC 1.

Errata.- "1900 values of $Y_{0}(x)$ and $Y_{1}(x)$ contain 20 errors." (L. J. CoMriE).

\section{A. H. HeATLEy}

1. Canadian J. Research, v. 19B, 1941, p. 270. [V].

2. R. So. Canada, Trans., Sect. III. Chem. Math. Phys. Sci., s. 3, v. 39, 1943, p. 26, 28. [VI].

M. VAN DER HELD

1. Z. f. Physik, v. 39, 1926, table, p. 840 (in an article by H. A. Kramers). [V].

T. HESSELBERG \& E. BJÖRKDAL

1. Beiträge z. Physik d. freien Atmosphäre, v. 15, 1929, p. 127. [III].

K. HesSENBERG, see JAHNKE \& EMDE $1_{2}$

K. HIDAKA

1. Kobe, Imp. Marine Observatory, Memoirs, v. 4, 1931, p. 114. [VI].

A. V. HILL

1. R. So. London, Proc., v. 104B, 1928, p. 71. [I].

A. P. Hillman, see Lowan

E. Hogner (see also AIry)

1. Arkivf. Mat. Astronomi o. Fysik, v. 17, no. 12, 1923, p. 34, 37, graphs, p. 34, 38. [V].

S. C. Hollister, see Hawkins

W. HORENSTEIN, see LOWAN

W. HORT

1. Z. techn. Physik, v. 1, 1920, p. 217-219. [III].

W. J. HUMphreys (see also Airy, SCHWERd)

1. Franklin Inst., J., v. 188, 1919, p. 481, 651, graph, p. 480. [I, V]. The first table and graph (p. 480-481) copied from PernTer, p. 515, 517, and the second table (p. 651) is Airy's table as in MASCART, p. $310,312$.

12. Physics of the Air, New York, McGraw Hill, 1920, p. 473-474, 531-532.

13. idem, second ed., 1929, p. 473-474, 531-533.

14. idem, third ed., 1940, p. 491-493, 549-551.

H. F. Hunt, see J. Perry

W. v. IgNATOWSKY

1. Ann.d. Physik, s. 4, v. 23, 1907, p. 895-900. [X, XII]. The tables of $C(u), S(u)$ are reprinted in JAHNKE \& EMDE $1_{1}$ (p. 26) - $1_{5}$ (p. 34). In $1_{6}$ (p. 34) 8 errors are corrected.

Y. IKEDA

1. Z. angew. Math. u. Mech., v. 5, 1925, p. 80-81. [I, V].

12. HAYASHI 2, p. 80, 100. 
J. G, ISHERWOOD

1. Manchester Lit. Phil. So., Memoirs, v. 48, 1904, no. 19. [III].

Tables: $I_{n}(x)$ and $K_{n}(x), n=0(1) 10$. The tables of $I_{n}(x)$ are abridged from Lodge 1 , and the tables of $K_{0}(x), K_{1}(x)$ from Aldis 1. $K_{n}(x), n=2(1) 10$, alone are original. J. IVORY

12. Gray \& Mathews $1_{2}$, T. XII (original part).

1. R. So. London, Trans., v. 128, 1838, p. 211-213. [IV].

J. G. JAEGER

1. R. So. Edinburgh, Proc., v. 61A, 1942, graph, p. 226. [X].

J. G. Jaeger \& M. Clarke

1. R. So. Edinburgh, Proc., v. 61A, 1942, p. 229-230. [X].

E. JAHNKE

1. Archiv Math. Phys., s. 3, v. 23, 1914, p. 265-267. [XII].

E. JAHNKE \& F. EMDE

1. Funktionentafeln mit Formeln und Kurven, Leipzig and Berlin, 1909; second, unchanged, reprint 1929 , p. 2-3, 104-164. $15.3 \times 23.6 \mathrm{~cm}$. [I-IV, VI, X, XI].

Tables: T. I, p. 106: least root of $J_{-1 / 3}(x)=0$ (GreenhILL, see $M T A C$, p. 196), tables of $-2^{3}\left(\frac{1}{4}\right) ! x^{-\frac{1}{3}} J_{1 / 4}(x / 2), 2^{-\frac{1}{1}}\left(-\frac{1}{4}\right) ! x^{\frac{1}{2}} J_{-1 / 4}(x / 2)$, from PRANDTL; T. II : graphs of $J_{n}(x)$, for $n= \pm .5, \pm 1.5, \pm 6.5$ and Tables for $\pm n=.5(1) 6.5$, from LoMmeL 3 ; T. III : $J_{0}, J_{1}$ graphs and tables, abridged from BESSEL and MEISSEL 1 ; T. IV: Zeros, $x_{n}$, of $J_{0}(x)$, and corresponding values of $J_{1}\left(x_{n}\right)$, from WILlson and PEIRCE; T. V: Zeros, $x_{n}$, of $J_{1}(x)$, and values of $J_{0}\left(x_{n}\right)$, from MeIsseL 2; value of $\sum_{n-1}^{\infty} 1 /\left[x_{n} J_{0}\left(x_{n}\right)\right]$ from Riemann \& Weber, Die partiellen Differential-Gleichungen der Math. Physik nach Riemann's Vorlesungen, v. 1, fourth ed. 1900, p. 477; fifth ed., 1910, p. 500; T. VI: graph, and Tables of $Y^{(0)}(x), Y^{(1)}(x)$, from B. A. Smith 1; T. VII: $K_{0}(x)$, $K_{1}(x)$ from Aldis 2 [who called them $G_{0}(x), G_{1}(x)$ ]; T. VIII: $K_{0}(x), K_{1}(x)$, from B. A. Sмiтh 2 [who called them $k J_{0}(x)-Y_{0}(x), k J_{1}(x)-Y_{1}(x)$ ]; T. IX: $Y_{0}(x)$, $Y_{1}(x)$, tables and graph; T. X: zeros of $Y_{0}(x)$ and $Y_{1}(x)$, from KALÄHNE 3; T. XI: $I_{0}(x) \equiv J_{0}(i x)$ from Aldis 1; T. XII: $I_{1}(x) \equiv-i J_{1}(i x)$ from Lodge 2, Aldis 1; T. XIII: $i H_{0}^{(1)}(i x)=-i H_{0}^{(2)}(-i x)= \pm i J_{0}( \pm i x)-Y_{0}( \pm i x) ;-H_{1}^{(1)}(i x)=$ $-H_{1}^{(2)}(-i x)=-J_{1}( \pm x)+i Y_{1}( \pm i x) ;$ T. XIV: $\frac{1}{2} i \pi H_{0}^{(1)}(i x), \frac{1}{2} \pi H_{1}^{(1)}(i x),\left[K_{0}(x)\right.$, $K_{1}(x)$ ], from ALDIs 1; T. XV-XVI: $J_{0}\left(x i^{1}\right)$ [ber $x$, bei $x$ ], ${ }^{1} J_{1}\left(x i^{3}\right)$, tables and graphs from Aldis 2; T. XVII-XVIII: $N_{0}\left(x i^{3}\right)\left[Y_{0}\left(x i^{3}\right)\right], H_{0}^{(1)}\left(x i^{3}\right), H_{1}^{(1)}\left(x i^{3}\right)$, tables and graphs; T. XIX: $\ln (y x / 2)$; T. $\mathrm{XX}$ : ber $x$, bei $x$, $\operatorname{ber}^{\prime} x$, bei' $x$ from MacleaN 1; T. XXI: $\frac{1}{2} q \sqrt{-i} J_{0}(q \sqrt{-i}) / J_{1}(q \sqrt{-i})$, from MACLEAN \& ZENNECK; T. XXII: BourgeT; T. XXIII: $J_{n}(1)$ to $J_{n}(24)$, abridgment to $4 \mathrm{~S}$ of MEISSEL 5, first printed in GRAY \& Mathews $1_{1}$; T. XXIV: $I_{n}(x), n=0(1) 11$, graphs and tables from LodGe 1; T. XXV: zeros of $I_{n}(x) J_{n}{ }^{\prime}(x)-J_{n}(x) I_{n}{ }^{\prime}(x)$, from Schulze; T. XXVIXXIX: roots of $J_{n}(x) Y_{n}(k x)-J_{n}(k x) Y_{n}(x)=0$, and values of $(k-1) x^{(w)}$, from KALÄHNE 2. Zeros of $J_{3 / 2}(x)$, and roots of $\cosh 2 x \cos 2 x \pm 1=0$ lor $J_{\frac{1}{3}}(x) I_{-\frac{1}{3}}(x) \pm I_{\frac{1}{3}}(x) J_{-\frac{1}{3}}(x)=0$, etc.] p. 3. Tables of $C(u), S(u)$ by IgNATowsky, p. 26.

Errata: P. 106 , zero of $J_{-1 / 3}(x)$, for 1.88 , read 1.866350 ; p. $122, J_{1}\left(x_{17}\right)$, for .1010 , read .1100 (see RMT 113); p. 137, 10 errors in signs in the imaginary parts from $x=5.1$ to $x=6.0$ inclusive; on p. 139 , in the real part of $x=6$, and on p. 140, in the real part of $x=2.4$. (F. F. BisaCre, Phil. Mag., s. 6, v. 45, p. 1037-1038); p. 147, T. XXII (see $M T A C$, p. 160). In T. VII-VIII, 18 values of $K_{0}(x)$, and 15 of $K_{1}(x)$, differ by a unit in the last place; there are two cases of $K_{1}(x)$ where the difference is two units.

12. Second (revised) edition, Leipzig and Berlin, 1933, p. 30-31, 192-318. $16 \times 24.1 \mathrm{~cm}$. [I-VIII, X, XI].

Tables: In what follows here no reference will be made to the 76 graphs and reliefs given in connection with the special tables of Bessel functions. T.[1]: $J_{n}(x)$, for 
$\pm n=.25(.25) .75,1.5(1) 6.5$; T.[2]: $J_{0}(x), J_{1}(x)$; T.[3]: Zeros, $x_{n}$, of $J_{0}(x)$ and corresponding values of $J_{1}\left(x_{n}\right) ;$ T.[4]: Zeros, $x_{n}^{\prime}$ of $J_{1}(x)$ and values of $J_{0}\left(x_{n}^{\prime}\right) ;$ T.[5-6]: first and second roots of $J_{p}(x)=0 ;$ T.[7]: T. XXII of first ed.; T.[8]: $J_{n}(1)$ to $J_{n}(24)$; T. [9]: $\Lambda_{0}(x)$ to $\Lambda_{8}(x)$ by A. WALTHER, S. Gradstein and K. HesSenberG; T.[10]: $N_{0}(x), N_{1}(x),\left[Y_{0}(x), Y_{1}(x)\right.$ ]; T.[11 to 14]; roots of $J_{n}(x) Y_{n}(k x)$ $-J_{n}(k x) Y_{n}(x)=0$; T.[15]: T. XI enlarged, and T. XII of $1_{1}$; T.[16]: $I_{n}(x)$ for $n=0(1) 11$; zeros of $J_{-n}(i x)$, from LoDGE 1; T.[17]: Zeros of $I_{n}(x) J_{n-1}(x)$ $+J_{n}(x) I_{n-1}(x) ;$ T.[18]: $I_{ \pm 1 / 8}(x), I_{ \pm 2 / 8}(x)$ from DiNNIK 9; T.[19]: as in $1_{1}$, T.XII; and zero of $H_{1}{ }^{(2) '}\left(r i^{\rho}\right)$, or of $H_{1}^{(2)}(z)=z H_{0}^{(2)}(z)$; T.[20]: ber $x$, bei $x$; T.[21]: ber' $x$, bei' $x$; T.[22]: $\operatorname{ReH}_{0}^{(1)}\left(x i^{3}\right), \quad \operatorname{Im} H_{0}^{(1)}\left(x i^{i}\right) ; \quad T$.[23]: $\operatorname{Re}^{i} H_{1}^{(1)}\left(x i^{3}\right)=-d \operatorname{ReH}_{0}{ }^{(1)}\left(x i^{1}\right) / d x$, $\operatorname{Im} i^{i} H_{1}^{(1)}\left(x i^{3}\right)=-d I m H_{0}^{(1)}\left(x i^{i}\right) / d x ; T$.[24]: $i^{n / 2} J_{n}\left(x i^{i}\right)=u_{n}+i v_{n}$ [ber $x$, bei $x$, $\operatorname{ber}^{\prime} x$, bei' $\left.x\right] ; i^{n / 2} H_{n}^{(1)}\left(x i^{\natural}\right)=U_{n}+i V_{n}$; T.[25]: $J\left(r i^{3}\right)=b i^{\beta}$ and $H^{(1)}\left(r i^{3}\right)=h i^{\eta}$; T.[26]: $J_{0}\left(r i^{\dagger}\right): J_{1}\left(r i^{\dagger}\right)$ and $H_{0}^{(1)}\left(r i^{\dagger}\right): H_{1}^{(1)}\left(r i^{i}\right)$. Tables of IGNATOWSKY, p. 109.

Errata: P. $237, n=17, J_{1}\left(x_{n}\right)$, for 0.1010 , read 0.1100 ; p. 239, see MTE 21 ; p. 248, $J_{0}(21)$, for -0.3175 , read -0.03175 ; p. 285 , see DiNnik 9.

$1_{3}-1_{5}$. Third (revised) edition, Leipzig and Berlin, 1938, p. 126-228. Fourth ed. 1940; fifth ed., 1943 see RMT $113.15 .3 \times 22.8 \mathrm{~cm}$. [I-XI].

Tables: In what follows here no reference will be made to the 81 graphs and reliefs in connection with the special tables of Bessel Functions. P. 154-155: $J_{n}(x)$ for $\pm n=.5(1) 6.5 ;$ p. $156-163: J_{0}(x), J_{1}(x) ;$ p. $164: J_{n}(x)$ for $\pm n=.25, .75 ;$ p. 166 : zeros of $J_{0}(x)$ and $J_{1}(x)$ and the corresponding values of $J_{1}(x)$ and $J_{0}(x) ;$ p. 167: first and second zeros of $J_{n}(x) ;$ p. 168: 54 roots of $J_{p}(x)=0$, T. XXII of first edition and 7 of second; p. 171-179: $J_{n}(1)$ to $J_{n}(29)$, the $J_{n}(25)-J_{n}(29)$ having been calculated by M. J. DITORo; p. 180-206: as in T.[9]-[14] of $1_{2} ;$ p. 212-217: Lommel-Weber Functions $\Omega_{0}(x), \Omega_{1}(x)$; p. 218-223; Struve function $S_{0}(x), S_{1}(x)$; p. 226-229: as in T. XI-XII of $1_{1} ;$ p. 232-233: as in T.16 of $1_{2} ;$ p. 234: zeros of

$J_{n}(i x) J_{n}{ }^{\prime}(x)-i J_{n}(x) J_{n}{ }^{\prime}(i x) ;$ p. $235-267$ : as in T.18-26 of $1_{2}$. Tables of IGNATOWSKy, p. 34.

Errata: P. $166, n=17, J_{1}\left(x_{n}\right)$, for .1010 , read .1100 ; p. 168, see MTE 21; p. 177 $J_{0}(21)$, for -0.3175 ; read -0.03175 ; p. 164,235 , see DinNik 8 and 9.

1. New York, Dover Publications, 1945. Revised by the correction of nearly 400 errors, and the addition to the Bibliography of 40 new titles.

J. JAW, see ERTEL

H. JEFFREYS, see BAASMTC 8

S. Johnston, see BAASMTC 9, HARTreE

C. W. JONES, see BAASMTC 9

W. F. DEJONG, see BOUMAN

J. JOUBERT, see MACLEAN

H. KADEN \& H. KAUFMANN

1. Elek. Nach. Tech., v. 15, 1938, graph p. 211. [XI].

A. KALÄHNE

1. Annalen d. Physik, s. 4, v. 19, 1906, p. 88-89, 92-94. [I, II, IV].

2. Z. Math. Phys., v. 54 , p. $68-85,1906$, [I, II, IV]. Tables on p. $68,69,81$, and a fig. on p. 79 are reproduced in JAHNKE \& EMDE, $1_{1}-1_{5}$; seven errors $1_{5}$ (p. 204-206) have been corrected in $1_{6}$.

3. Grundzüge der Mathematisch-physikalischen Akustik, v. 2, Leipzig and Berlin, 1913, graphs, p. 161-162. [I, II].

4. Annalen d. Physik, s. 4, v. 46, 1915, p. 36-37. [II].

J. C. Kapteyn \& P. J. van Rhijn

1. Groningen, Steerekundig Laboratorium, Publications, no. 30, 1920, p. 63. [III].

12. SMart 1, p. 219.

K. KARAS

1. Z. angew. Math. Mech., v. 16, 1937, p. 249-251. [V, VI]. Tables: T. $1: J_{ \pm 2 / 8}(x), Y_{ \pm 2 / 3}(x)$; T. $2: J_{ \pm 1 / 4}(x), Y_{ \pm 1 / 4}(x)$; T. $3: J_{ \pm 3 / 4}(x), Y_{ \pm 3 / 4}(x)$. 
Errata: The NYMTP has checked the entries for $J_{ \pm 2 / 3}(x), J_{ \pm 1 / 4}(x)$, and $J_{ \pm 3 / 4}(x)$, and submitted to us a list of 108 errors, 90 of a unit in the last decimal place and 18 of two or more units. The worst errors are the following: $J_{1 / 4}(7.9)$, for .2591 , read $.2582 ; J_{1 / 4}(8)$, for .2449 , read $.2436 ; J_{-1 / 4}(0.2)$ for 1.4310 , read $1.4319 ; J_{-3 / 4}(0.7)$, for .3277 , read .3234 .

H. KaUfMaNN, see KaDEN

G. Kennedy, see Proudman

A. E. Kennelly, F. A. Laws, \& P. H. Pierce

1. A.I.E.E., Trans., v. 34, 1915, p. 1994-1999. [XI].

12. A.I.E.E., Proc., v. 34, 1915, p. 1790-1795.

J. KERN

1. Annalen d. Physik, s. 4, v. 42, 1913, p. 475-477. [I].

I. Kimura, see Sakuraba

L. V. KING

1. R. So. London, Trans., v. 214A, 1914, p. 412-413. [III, X].

G. KIRchHofF

1. J.f. d. reine u. angew. Math., v. 40, 1850, p. 85. [III].

12. Gesammelte Abhandlungen, Leipzig, 1882, p. 276. From the solutions $\log \left(\frac{1}{2} x\right)^{4}$, to $5 D$, of Kirchhoff's equation, for $\sigma=1 / 4$ and $1 / 3$, Prescotr, p. 597 gave the values of $x^{4}$, to $5 \mathrm{~S}$.

2. Annalen d. Physik, v. 81,1850 , p. 260. [III]. This article is a resumé of $1_{1}$ but contains more detailed numerical results and still further theoretical calculations. The table for $\sigma=1 / 4$ is reprinted in SchulzE, p. 799.

2. Gesammelte Abhandlungen, p. 282.

W. J. Kirkham, see H. T. Davis

H. KLEINERT

1. o Zur Theorie des Purpurlichtes, Diss. Bern, 1921. [IV]. Also Blumer 2, p. 309.

H. KÖHLER

1. Arkivf. mat. o. Fysik, v. 24A, no. 9, 1934, p. 24. [I].

K. M. KOHLER

1. Ms. table of $\operatorname{yer}_{1}(x)$ real part of $Y_{1}(x \sqrt{-i})$, and of yei $i_{1}(x)$ the imaginary part, for $x=[0(.2) 6 ; 5 \mathrm{D}]$, with curves, in the Institute for applied Math., Univ. Berlin. (R. v. MISEs, Verzeichnis berechneter Funktionentafeln, part 1, Berlin, 1928, p. 19.) $[\mathrm{XI}]$.

H. KoHN

1. Physikal. Z., v. 15, 1914, p. 104, tables and graphs. [III].

V. A. Kolpinsky, see For

H. A. Kramers (see also Held)

1. Phil. Mag., s. 6, v. 46, 1923, graphs p. 847,851 . [V].

H. G. KÜSSNER

1. Luftfahrtforschung, v. 17, 1940, p. 374, 376, 378. [III, X].

12. English translation, Nat. Advisory Comm. f. Aeronautics, Techn. Memorandums, no. 979,1941 , tables on plate facing p. [28].

J. W. LABUS

1. Institute of Radio Engineers, Proc., v. 23, 1935, graph, p. 941. [X].

R. LADENBURG \& F. ReICHE

1. Annalen d. Physik, s. 4, v. 42, 1913, p. 202-203. [III].

H. LAMB

1. London Math. So., Proc., v. 13, 1882, p. 197. [IV].

2. R. So. London, Trans., v. 174, p. 530, 1883. [IV].

3. London Math. So., Proc., v. 15, 1884, p. 143. [I].

3. Tномson, p. 357.

4. London Math. So., Proc., v. 15, 1884, p. 272. [I]. 
5. London Math. So., Proc., s. 2, v. 2, 1904, p. 393-396, graphs, p. 397-400. [XII].

6. R. So. London, Proc., v. 84 A, 1910, p. 565. [I].

7. R. So. London, Proc., v. 93A, 1917, p. 303-304, 309. [X].

W. E. LAMB, see NoRDSIECK

W. LÁsKa, see LOMMEL, STOKES

F. A. Laws, see KenNelly

M. LEE, see HaWkins

D. H. LEHMER

1. $M T A C$, v. 1,1944 , p. 135. [III].

Erratum: $I_{100}(75)$ is here given to 10D. J. C. P. MiLler has shown that the result, incorrect after 3D, should be 134001.44891 20951 594. Details in this regard appear in our next issue.

2. $M T A C$, v. 1, 1944, p. 196. [V].

A. LEVASSEUR

1. J. Physique et Radium, s. 7, v. 1, 1930, p. 96. [XI].

A. LODGE

1. B.A.A.S., Report, 1889, p. 28-32. [III]. Compare Dwight 5 and JahnKe \& Emde $1_{1}$. An abridgment in DALE, p. 82, IsheRwOOD, and TAKAgI, p. 76.

12. Gray \& Mathews $1_{1}, \mathrm{~T}$. VI; $1_{2}$, T. IX.

2. B.A.A.S., Report, 1893, p. 227-279. [III, XI].

2. GRAY \& MATHEWS $1_{1}$, T. IV; $1_{2}$, T. VI.

3. B.A.A.S., Report, 1896, p. 99-149. [III].

4. B.A.A.S., Report, 1907, p. 94-95. [II, III, IV].

5. B.A.A.S., Report, 1909, p. 33, 35. [II, IV].

E. C. J. v. LoMmel (see also P. A. HANSEN)

1. Z. Math. Phys., v. 15, 1870, p. 151-155, 164-169. [I]. Among various tables here given is one, of the values of the first six zeros of $J_{1}(x)$, which is also given, in abbreviated form in W. LÁSKA, Sammlung von Formeln der reinen und angewandten Mathematik, zweite Lieferung, Brunswick, 1889, p. 390.

2. Bayer. Akad. d. Wissen., math. natw. Abt., Abh., v. 15, 1886, p. 312-315, 323, 328. [I, XII]. The 6-place table of $j_{2, s}$, on p. 315 (in somewhat abbreviated form on p. 167 of no. 1, 1870) has been also reproduced in Gray \& Mathews $1_{1}$, p. $180 ; 1_{2}$, p. 192. The whole table of $j_{2,8} / \pi, 2 J_{1}\left(j_{2, s}\right) / j_{2,8}, 4 J_{1}{ }^{2}\left(j_{2, s}\right) / j^{2} 2, s$ is reproduced in E. E. N. Mascart, Traité d'Optique, v. 3, Paris, 1893, p. 668.

Errata: Comparison of the table of $j_{2,8}$ with Watson 3, p. 749 shows that, for the first 4 values of $s$, the last digits are in error by 8 to 16 units. There are corresponding errors in the table for $j_{2,8} / \pi$.

3. Bayer. Akad. d. Wissen., math. natw. Abt., Abh., v. 15, 1886, p. 644-651. [IV, X, XII]. Errata: AIREy 26 pointed out the unreliability of Lommel 3, p. $648(S(u)$ and $C(u))$ in last figures. Comparison with AIREY up to $x=20$, showed 14 errors in LoMmeL, two of them serious: $x=.2$, for 0.023788 , read $0.023720 ; x=1.5$, for 0.415348 , read $0.415483 ; 9$ of the errors were unit errors in the last figure [at $x=15,15.5,17.5$ in $C(u)$; and at $x=15.5,13.5,12,11.5,8,6.5$ in $S(u)$.

J. W. Wrench, Jr. recalculated, to $8 \mathrm{D}$, the rest of the table, $20 \leq x \leq 50$. He found that of the 18 further errors 17 were unit errors in the last figure and one of 2 units-in $C(u), x=47.5$, for .479313 , read .479311 . The 17 unit errors are in $C(u), x=20.5,21,21.5,25.5,34,34.5,38.5,48.5$; and in $S(u), x=20.5,21,24$, $28.5,34,36.5,39.5,40.5,44.5$. Practically all errors noted throughout the table, including the worst ones, are also to be found in WATSON 3, which reproduces this table.

A. N. LOWAN, see BAASMTC 8, DinNik 11, and NYMTP

A. N. Lowan \& M. Abramowitz

1. J. Math. Physics, M.I.T., v. 22, 1943, p. 3-12. [X].

A. N. Lowan, G. Blanch, \& M. Abramowitz

1. J. Math. Physics, v. 22, 1943, p. 54-56. [X]. 
A. N. Lowan \& A. P. Hillman

1. J. Math. Phys., M.I.T., v. 22, 1943, p. 208-209. [II]. This publication was first distributed in 1941 by the NYMTP as a 2-sheet hectograph Short Table, in size $20.4 \times 26.8 \mathrm{~cm}$.

A. N. Lowan \& W. Horenstein

1. J. Math. Phys., M.I.T., v. 21, 1942, p. 273-274. [IV].

A. E. LUdLAM, see Topping

F. LÜDI

1. Helvetica Physica Acta, v. 9, 1936, graph p. 667. [I].

H. M. MaCDONALD

1. R. So. London, Proc., v. 90A, 1914, p. 54. [V].

12. G. N. Watson, R. So. London, Proc., v. 95A, 1918, p. 96.

N. W. McLachlan

1. Phil. Mag., s. 7, v. 7, 1929, graph p. 1023. [IX].

2. Bessel Functions for Engineers, Oxford, Clarendon Press, 1934, p. 17, 173-183, graphs p. 9, 21 and 50. [I-III, IX, XI].

Tables: p. 173-183: T. 1: $J_{0}(x)$; T. $2: J_{1}(x)$; T. 3: $Y_{0}(x)$; T. 4: $Y_{1}(x)$; T. 5: $J_{n}(x)$, $n=2,3,4$; T. $6: H_{0}(x)$; T. $7: H_{1}(x)$; T. 8: ber $x$, bei $x$; T. 9: ber' $x$, bei' $x$; T. 10: ker $x$, kei $x ;$ T. 11: $\operatorname{ker}^{\prime} x, \operatorname{kei}^{\prime} x ;$ T. 12: $\operatorname{ber}_{n} x, \operatorname{bei}_{n} x, \operatorname{ber}_{n}{ }^{\prime} x, \operatorname{bei}{ }^{\prime} x ;$ T. 13: $\operatorname{ker}_{n} x$, $\operatorname{kei}_{n} x, \operatorname{ker}_{n}{ }^{\prime} x$, $\operatorname{kei}_{n}{ }^{\prime} x$; T. 14: ber $x+i$ bei $x=M_{0}(x) e^{i \theta_{0}(x)}$; T. 15: $\operatorname{ber}_{1} x+i$ bei $_{1} x$ $=M_{1}(x) e^{i \theta_{1}(x)}$.

N. W. McLachlan \& A. L. Meyers

1. Phil. Mag., s. 7, v. 18, 1934, p. 621-624, tables checked by L. J. Comrie. [XI].

N. W. McLachlan \& G. A. V. Sowter

1. Phil. Mag., s. 7, v. 11, 1931, p. 53-54. [I, IX].

M. Maclean (see also Fleming)

1. Institution Electr. Engineers, J., v. 18, 1889, p. 36. [XI].

12. W. Thomson, Math. Phys. Papers, Cambridge, Univ. Press, v. 3, 1890, p. 493. In this reprint are 24 changes of numbers in the original table besides the addition of the values of the 6 functions for 30 , and of $W / V$ for $\infty$.

13. E. É. N. MASCART \& J. Joubert, Leşons sur l'Electricité et le Magnétisme, second ed., Paris, v. 1,1896, p. 718 ; a reprint of $1_{2}$ omitting $W / V$, with 3 changes none of which is correct.

14. Abridgement, ber $x$, bei $x$, $\operatorname{ber}^{\prime} x$, bei' $x$, with additional errors, JAhNKe $\&$ EMde $1_{1}$, 1909 , p. 146, and WeINREICH, p. 46.

16. Reprint from $1_{2}$ of tables of $\operatorname{ber}^{\prime} x$, bei' $x$, to $4 \mathrm{~S}$, with 10 changes $(x=5$ to 30 ), 5 correct, 5 incorrect: A. RusSell, A Treatise on the Theory of Alternating Currents, v. 1, Cambridge, Univ. Press, second ed., 1914, p. 231. The accuracy of Maclean's Table was discussed by Russell in Phil. Mag., s. 6, v. 17, 1909, p. 533-535; and in Phy. So. London, Proc., v. 21, 1910, p. 591-593.

Errata: This table of MACLEAN has been completely recalculated and checked by J. W. WRENCH, Jr. who has made the following report on the 41 errors in the table as published in $1_{2}$ above:

ber $q$ : unit errors in last digits at $q=2.5,5.5 ; q=20$, for 47489.2 read 47489.4; $q=30$, for -39630000 , read -46117603

bei $q$ : unit errors at $q=2.5,3.5,4.5,20 ; q=5.5$, for -2.7902 , read -2.7890 ; $q=30$, for 110240000 , read 109955713

ber' $^{\prime} q: q=5$, for -3.8442 , read $-3.8453 ; q=8$, for 38.294 , read $38.311 ; q=10$, for 51.207, read 51.195; $q=15$, for 91.061, read 91.055; $q=30$, for -109330000 , read -109598679

bei' $q$ : unit errors at $q=10,20 ; q=5$, for -4.3538 , read $-4.3541 ; q=8$, for -7.6615 , read $-7.6603 ; q=30$, for 45980000 , read 43292237

$W / V$ : unit errors at $q=5,10 ; q=.5$, for 4.0000 , read $4.0013 ; q=1$, for 2.0113 , read $2.0104 ; q=2$, for 1.0805 , read $1.0782 ; q=2.5$, for .9398 , read $.9403 ; q=8$, for .7739 , read $.7736 ; q=30$, for .7251 , read .7241 
$x W / 2 V$ : unit errors at $q=3,4,5.5,6,15 ; q=.5$, for 1.0000 , read $1.0003 ; q=1$, for 1.0056 , read $1.0052 ; q=2$, for 1.0805 , read $1.0782 ; q=2.5$, for 1.1747 , read 1.1754; $q=5$, for 2.0430, read 2.0427; $q=8$, for 3.0956, read 3.0944; $q=10$, for 3.7980 , read 3.7986; $q=30$, for 10.8765 , read 10.8610 .

Editorial Note: Russell's discussions referred to above present extraordinary misrepresentations later accepted by many people. He suggests that Maclean's tables in $1_{1}$ and $1_{2}$ are identical and consistently quotes the results only as in $1_{1}$ ! He misrepresents also by introducing results of MASCART \& Joubert. Of his 12 calculated values of ber $x$, bei $x$, ber $^{\prime} x$, bei' $x(x=10,15,20) 10$ are incorrect. In conclusion it may be put on record that WRENCH found the following results: ber $15=-2967.25453 \frac{5}{4}$, bei $15=-2952.707888$, ber' $15=91.05533129$, bei' $^{\prime} 15=-4087.7552386$

M. Maclean \& J. Zenneck

1. T. XXI, p. 147, in JAhnke \& Emde $1_{1}$. The function tabulated is the reciprocal of that tabulated by W. Fischer. [XI].

A. R. MCLEOD

1. Phil. Mag., s. 6, v. 37, 1919, p. 140-143. [I].

T. M. MacRobert, see Gray \& Mathews

I. MALKIN

1. Franklin Inst., J., v. 234, 1942, p. 368. [III].

N. N. MaLow

1. Acad. Sci. U.S.SR., J. of Physics, v. 4, 1941, p. 476. [I].

E. E. N. Mascart (see also Lommel, Maclean)

1. Traité d'Optique, Paris, v. 1, 1889, p. 310-312, 397, 408. [I, V].

12. Mascart here reproduces the following tables: AIRY 2 (p. 310); SchwERD (p. 311); Airy 5 (p. 397); RAYLEIGH 4 (p. 408).

H. S. W. Massey, see D. R. Bates

Mathematical Tables Project, New York, see New York, Mathematical Tables PROJECT

G. B. Mathews, see Gray

R. H. D. Mayall (see also Fresnel)

1. Cambridge Phil. So., Proc., v. 9, 1897, p. 268-269. [X, XII]

E. MEISSEL

1. Akad. d. Wissen., Berlin, Math. Abhandlungen vom Jahre 1888, 1889, 23 p. $21.7 \times 27.5 \mathrm{~cm}$. [I]. Also published separately, Tafel der Bessel'schen Functionen $I_{k}^{\circ}$ and $I_{k}^{1}$ von $k=0$ bis $k=15.5$ berechnet. See BAASMTC 1 .

Errata: 1. $J_{0}(.62)$ for .90518 , read $.90618 ; 2$. $J_{0}(1.71)$ for .39320 , read $.39220 ; 3$. $J_{0}(1.89)$ for .28663 , read $.28763 ; 4 . J_{1}(7.87)$ for .21401 read .21407 . Nos. $1,3,4$ were announced by Airey 26, p. 297; and no. 2 by Meissel (Gray \& Mathews 11, p. 245).

12. Gray \& Mathews, $1_{1}$, p. $247 f ; 1_{2}$, p. $267 f$.

Errata: see under GRAY \& Mathews.

2. Ueber die Bessel'schen Funktionen $I_{k}^{\circ}, I_{k}^{1}$, (Progr.), Kiel, 1890, p. 4. [I]. $19.7 \times 24.9$ $\mathrm{cm}$.

22. The first 50 roots of $J_{1}(x)=0$ and corresponding max. or min. values of $J_{0}(x)$ to $16 \mathrm{D}$, in Gray \& Mathews $1_{1}$, p. $280 ; 1_{2}$, p. 301.

2. Davis \& KirkhaM, p. 769f. 10-place abridgment. Erratum: For all three reprints in $x_{9}{ }^{\prime}$, for 29.0468285340 , read 29.04682 85349; the original was correct, contrary to what Comrie states (BAASMTC 1, p. xiii).

3. Astr. Nach., v. 128, 1891, col. 153-156. [I]. The tables here are (1), a 6-place extract from no. 5 ; (2), $J_{n}(1000)$. In col. $151, J_{20}(20)$ is given.

4. Astr. Nach., v. 129,1892 , cols. 283-284. [I].

5. Tables of $J_{n}(x), x=1$ to 24 , in Gray \& Mathews $1_{1}$. p. 266-279; $1_{2}$, p. 286-299. [I]. Abridged edition in JAHNKE \& EMDE 1 1 $-1_{5}$. 4-place abridgement of $J_{18}(x), J_{21}(x)$, 
$x=11(1) 24$, by Rayleigh, Phil. Mag., s. 6, v. 20, 1910, p. 1002, Scientific Papers, v. 5, 1912, p. 618. See H. T. Davis.

K. Menges

Errata: see under Gray \& Mathews.

1. Akustische $Z$., v. 6, 1941, p. 105. [IX].

M. W. Meres, see Muskat

A. L. Meyers, see McLachlan

A. G. M. MICHELL

1. Phil. Mag., s. 5, v. 48, 1899, p. 302-303, 305. [VI].

2. Z. Math. Phys., v. 52, 1905, p. 130. [III, IX].

G. MIE

1. Annalen d. Physik, s. 4, v. 25, 1908, p. 420-426, 428-432, 438, 440-441. [IV].

W. Miehle, see Corrington

J. C. P. Miller (see also BAASMTC 3, 4, 8, 9, Lehmer 1, Muskat)

1. [Tables of Airy Integrals], to be published by BAASMTC in 1944 (?). For contents, see BAASMTC 8. [II, III, V].

W. E. MILLER

1. General Electric Rev., v. 12, 1909, p. 228, graphs, p. 221-222. [XI].

W. L. MILLER \& A. R. Gordon

1. J. Phys. Chem., v. 35, 1931, p. 2873. [XII].

E. A. Milne \& R. H. FowleR

1. R. So. London, Proc., v. 98A, 1920, p. 419-421. [IX].

R. v. Mises, see Blumer, W. Fischer, Kohler, Tveritin

F. MORGAN, see MUSKat

P. M. MORSE

1. Vibration and Sound, New York and London, McGraw Hill, 1936, p. 153, 155, 159, 175, 333-335, 337-339. [I-IV, IX].

P. M. Morse \& Eva Haurvitz

1. Ms. Tables of Bessel Functions for spherical coordinates, prepared at M.I.T., Cambridge, Mass., in 1940. Computations were discontinued when word came of the more ambitious project NYMTP 9. About 20 hectograph copies of these tables ( 9 p.) were privately distributed but they were never otherwise published (except, perhaps MORSE 1, p. 339). The tables are as follows: (a) $j_{n}(x)$, for $n=0, \pm 1$, $\pm 2, \cdots, \pm 8, x=[0(.1) \cdot 2(.2) 9 ; 5 \mathrm{D}] ;$ (b) $j_{n}(x), n_{n}(x), D_{n}, D_{n}{ }^{\prime}, n=[0(1) 2 ; 4 \mathrm{~S}]$, and $\delta_{n}, \delta_{n}{ }^{\prime}$ to nearest $1^{\prime}$; (c) $j_{n}(x), n=0(1) 9, x=[0(.1) 6 ; 5 \mathrm{D}]$. [IV].

F. R. Moulton

1. Astrophys. J., v. 53,1921 , p. 257. [VII].

R. MÜLLER

1. Ueber die zahlenmässige Beherrschung und Anwendung einiger den Besselschen verwandten Funktionen nebst Bemerkungen zum Gebiet der Besselfunktionen (Diss. Techn. Hochschule, Berlin), 1939. [X].

12. Z. angew. Math. Mech., v. 19, 1939, p. 52-53.

W. MÜLLER

1. Annalen d. Physik, s. 5, v. 25,1936 , graphs, p. $189,194,195,201,203$. [XII].

M. Muskat, F. Morgan \& M. W. Meres

1. J. Applied Physics, v. 11, 1940, p. 212. [II].

Errata: J. C. P. MiLler found that the table was not wholly reliable.

\section{H. NAGAOKA}

1. Tokyo, University, College of Science, J., v. 4, 1891, p. 315. [I]. The 6-place table here of $J_{0}(n \pi), n=1(1) 50$, is abridged to 5 places in HAYASHI 2, p. 99.

2. Phil. Mag., s. 5, v. 45, 1898, p. 9. [1].

3. Astrophy. J., v. 51, 1920, p. 82(T. I), 92(T. IV), 95(T. VII), and p. 86-87(T. II-III). [X]. 
3. Handbuch der Astrophysik, v. 21, Grundlagen der Astrophysik, part 2, Berlin, 1929; "Theoretische Photometrie," by E. Schoenberg, p. 122, 123, 125 (T. I-III, VII of NAGAOKA).

\section{H. A. NANCARROW}

1. Phys. So., Proc., v. 45, 1933, p. 465, graphs p. 466-468. [I]. This title was suggested by the Liverpool Index.

J. NAYLER, see BICKLEY

New York Mathematical Tables Project $=$ NYMTP $=$ Mathematical Tables

Project, New York, Arnold N. Lowan, technical director (see also Lowan \& Hillman)

1. A Short Table of Bessel Functions for Complex Arguments, New York, 1939, 6 leaves, $21.5 \times 33 \mathrm{~cm}$., mimeographed on one leaf side. [I, III, XI]. $J_{0}\left(r e^{i \theta}\right)=U_{0}(r, \theta)+i V_{0}(r, \theta), r=[0(1) 10 ; 15 \mathrm{D}], \theta=0\left(5^{\circ}\right) 90^{\circ}$.

2. A Short Table of Spherical Bessel Functions to Five Significant Figures, New York, 1940, 12 leaves, $21.5 \times 33 \mathrm{~cm}$., mimeographed on one leaf side. [IV]. $j_{n}(x)=\left[(\pi / 2 x)^{1} J_{n+3}(x), \pm n=1(1) 10 ; x=[0(.1) 10 ; 5 \mathrm{~S}]\right.$.

3. Table of the Bessel Functions $J_{0}(z)$ and $J_{1}(z)$ for Complex Arguments, New York, Columbia University Press, 1943, xliv, 403 p. $19.6 \times 26.5 \mathrm{~cm}$. [I, III, XI].

Tables: $z=r e^{i \theta}, r=[0(.01) 10 ; 10 \mathrm{D}], \theta=0\left(5^{\circ}\right) 90^{\circ}$; table of five-point Lagrangean interpolation coefficients. See $M T A C$, p. 187-189.

Errata: C. R. Cosens, of the University of Cambridge Engineering Laboratory, pointed out that there are numerous errors in labelling the "Contour Lines" on p. $\mathrm{xv}, \mathrm{xvii}$. Details in this regard are given under MTE in $M T A C$ no. 8, p. 326.

4. "Table of $f(x)\left[=\Lambda_{n}(x)\right]=n !(x / 2)^{-n} J_{n}(x), " J$. Math. Physics, M.I.T., v. 23, 1944, p. 45-60. [VII].

The following Manuscripts are in possession of the Project (it is indicated when film copies are at Brown University):

5. Mss. - The first 18 derivatives with respect to $r$ of $J_{0}(z)$, and $J_{1}(z)$, where $z=r e^{i \theta}$, for $r=0(.1) 10, \theta=0\left(5^{\circ}\right) 90^{\circ}$, to a number of places (varying from 8 to 15 ) adequate for subsequent subtabulation of the functions to about 12D. See $M T A C$, p. 163. [I, XI].

6. Ms.-Table of Bessel Functions of the second kind $Y_{0}(z)$ and $Y_{1}(z)$ for complex arguments, $z=r e^{i \theta}, r=[0(.01) 10 ; 10 \mathrm{D}], \theta=0\left(5^{\circ}\right) 90^{\circ}$.

$Y_{0}(i x)=(-2 / \pi) K_{0}(x)+i I_{0}(x), \quad Y_{1}(i x)=-I_{1}(x)+i(2 / \pi) K_{1}(x)$. See $M T A C$, p. 165. There is a film of this Ms. at Brown University. [II, III, XI].

7. Ms. - The first 18 derivatives with respect to $r$ of the functions

$S_{0}(z)=Y_{0}(z)-(2 / \pi) J_{0}(z)(\ln z+\gamma), z=r e^{i \theta}$, and

$S_{1}(z)=Y_{1}(z)-(2 / \pi) J_{1}(z)(\ln z+\gamma)+2 / \pi z, r=0(.1) 10, \theta=0\left(5^{\circ}\right) 90^{\circ}$. These derivatives are given to a number of places adequate for subsequent subtabulation of the functions to $12 \mathrm{D}$. See $M T A C$, p. 164 [XI].

8. Mss.-Tables of $K_{0}(x)=E_{0}(x)+F_{0}(x) \log x, K_{1}(x)=E_{1}(x) / x+F_{1}(x) \log x$, for $x=[0(.01) 5 ; 8 \mathrm{~S}]$, with second differences for $x>.3$. For $x<.3$, the auxiliary functions $E_{0}(x), E_{1}(x), F_{0}(x), F_{1}(x)$ are also tabulated for $x=0(.001) .03$; and $K_{0}(x)$ and $K_{1}(x)$ for $x=0(.0001) .03(.001) 1$. All entries are given to $7 \mathrm{~S}$ with $\Delta^{2}$. See $M T A C$, p. 165. [III].

9. Ms. - Table of Spherical Bessel Functions, $j_{n}(x)=(\pi / 2 x)^{1} J_{n+3}(x)$ for $n=0, \pm 1$, $\pm 2, \cdots, \pm 21, x=[0(.01) 10 ; 8 \mathrm{~S}-10 \mathrm{~S}]$ (later extended for $n<-21, n\rangle+21$, $x>10$ ), with second and fourth central differences. [IV]. There is a film of this at Brown University. See $M T A C$, p. 92-93.

10. Mss. - Tables of $J_{n}(x)$ and $I_{n}(x)$ for $n= \pm 1 / 3, \pm 2 / 3, \pm 1 / 4, \pm 3 / 4, x=[0(.01) 25$; $10 \mathrm{D}$. Also the values of $u_{n}(x)$ and $v_{n}(x)$ in the asymptotic expansion of these functions in the form $u_{n}(x) \cos \left(x-\frac{1}{2} n \pi-\frac{1}{4} \pi\right)+v_{n}(x) \sin \left(x-\frac{1}{2} n \pi-\frac{1}{4} \pi\right)$ for $x$ ranging from 25 to about 30000 , at various intervals depending on the ease of interpolation, to $10 \mathrm{D}$. There are also values of $J_{ \pm n}(x)$ and $I_{n}(x)$, for $x=[10(1) 25 ; 13 \mathrm{D}]$; also for $I_{ \pm n}(x), x=[10,11,12,13 ; 13 \mathrm{~S}]$. [V, VI]. See $M T A C$, p. 93. 
11. Ms. -The first 15 derivatives of $J_{n}(x)$ and $I_{n}(x)$ for $n= \pm 1 / 3, \pm 2 / 3, \pm 1 / 4, \pm 3 / 4$, and $x=[10(.1) 25 ; 13 \mathrm{D}]$. These derivatives are given to a number of places adequate for the subsequent subtabulation of the functions to about $12 \mathrm{D}$ or $12 \mathrm{~S}$. [V, VI]. See $M T A C$, p. 93, 163.

12. The first 8 zeros of $J_{n}(x)$ for $n= \pm 1 / 3, \pm 2 / 3, \pm 1 / 4, \pm 3 / 4$, to $10 \mathrm{D}$. [V, VI]. There are films of most of the mss. nos. 10-12 at Brown University.

13. Mss. - The first 18 derivatives of $x^{-n} J_{n}(x)$ and $x^{-n} I_{n}(x)$ for $n= \pm 1 / 3, \pm 2 / 3$, $\pm 1 / 4, \pm 3 / 4$, and $x=0(.1) 10$. These derivatives are given to a number of places adequate for the subsequent subtabulation of the functions to about $12 \mathrm{D}$. [V, VI]. See $M T A C$, p. 163.

A. B. Newman \& A. H. Church

1. A.S.M.E., Trans., v. 57, 1935, p. A97-A98. [I, XII].

\section{J. W. Nicholson}

1. Phil. Mag., s. 6, v. 18, 1909, p. 16. [V]. This title was suggested by the Liverpool Index, where it is indicated that Nicholson's tables are unreliable.

2. London Math. So., Proc., s. 2, v. 11, 1912, p. 113-117, 122-123. [I, II].

Y. NOMURA

1. Sendai, Japan, Tôhoku teikoku daigaku, Science Reports, s. 1, math., v. 28, 1940, p. 316-318. [X].

2. Sendai, Japan, Tôhoku teikoku daigaku, Science Reports, s. 1, math., v. 29, 1940, p. 32-35. [X].

3. Nippon Sugaku-buturigakkwai, Tokyo, Proc., s. 3, v. 23, 1941, p. 173. [X].

A. Nordsieck, W. E. LAmb, \& G. E. UhLENBEck

1. Physica, v. 7, 1940, graphs p. 351-352. [X].

NYMTP $=$ New York Mathematical Tables Project

T. OKaya

1. Japanese J. Physics, v. 12, 1937, p. 13. [III, IV].

2. Nippon Sugaku-buturigakkwai, Tokyo, Proc., s. 3, v. 21, 1939, p. 293-298. [III].

2. Osaka, Imp. Univ., Faculty Sci., Collected Papers, s. B, Physics, v. 7, 1939, no. 3.

T. OKaYa \& T. TOBIISI

1. Nippon Sugaku-buturigakkwai, Tokyo, Proc., s. 3, v. 21, 1939, p. 777. [III].

12. Osaka, Imp. Univ., Fac. Sci., Collected Papers, s. B, Physics, v. 7, 1939, no. 17.

F. OLLENDORFF

1. Archiv f. Elektrotechnik, v. 17, 1926, p. 98, 101, and graphs, p. 83, 91. [X].

2. Archiv f. Elektrotechnik, v. 23, 1929, p. 163-164, graphs, p. 165-167. [III].

F. OllendorfF \& R. Seeliger

1. Physik. Z., v. 33, 1932, p. 518. [XII].

F. C. W. Olson \& O. T. Schultz

1. Industrial and Engineering Chemistry, v. 34, 1942, p. 876. [I, XII].

R. G. OLsson

A. ONo

1. Ingenieur Archiv, v. 8, 1937, p. 451-452. [II].

1. Phil. Mag., s. 6, v. 42, 1921, p. 1021. [VI].

S. P. OwEN

1. Phil. Mag., s. 6, v. 47, 1924, p. 736. [IX, X].

E. T. PARIS

1. Phil. Mag., s. 6, v. 30,1915 , p. 465,471 . [IV].

K. Pearson, see David, Elderton

P. O. Pedersen

1. Jahrb. d. dratlosen Telegraphie u. Telephonie, v. 4, 1911, p. 504, 505. [XI].

B. O. Peirce (see also Willson)

1. Am. Acad. Arts Sci., Proc., v. 41, 1906, p. 513, 515, 518, 521. [I]

12. Math. Phys. Papers, Cambridge, Harvard Univ. Press, 1926, p. 59, 61, 64, 66.

2. Am. Acad. Arts Sci., Proc., v. 43, 1907, p. 173-180. [I].

2. Math. Phys. Papers, 1926, p. 172-179. 
3. Am. Acad. Arts Sci., Proc., v. 46, 1911, p. 578. [I].

3. Math. Phys. Papers, p. 360-361.

J. M. Pernter (see also Airy, Humphreys, Schwerd)

1. Meteorologische Optik, Vienna and Leipzig, part 3, 1906, p. 440-442, 452-453, 515-517. [I, V].

J. Perry \& H. F. Hunt

1. Phil. Mag., s. 5, v. 40,1895 , p. $508-509$. [I].

R. L. Perry \& W. P. Berggren

1. Univ. of California, Publications in Engineering, v. 5, 1944, p. 70, 72. [X].

V. Petrž́llka

1. Annalen d. Physik, s. 5, v. 23, 1935, p. 160. [I].

J. PICHT

1. Annalen d. Physik, s. 4, v. 77, 1925, p. 752-755. [I].

P. H. Pierce, see Kennelly

F. Prola

1. Accad. d. Lincei, Cl.d. sci. fis. mat. e naturali, Rendiconti, v. 16 ${ }_{1}, 1907$, p. 41-42. [XI].

2. Nuovo Cimento, s. 5, v. 20, 1910, p. 29-34, tables and graphs. [XI].

H. Poncin

1. J. de Math. pures et appl., s. 9, v. 19, 1940, p. 173. [I].

L. PrandtL

1. o Kipp-Erscheinungen, Ein Fall von instabilem elastischen Gleichgewicht, (Diss. Munich), Nürnberg, 1901, p. 25. [VI].

12. JAHNKE \& EMDE 11, p. 106.

J. Prescott (see also Bourget, KirchHoff)

1. Applied Elasticity, London and New York, Longmans, 1924, p. 486, 488, 510, 587, 596, 597, 653. [I, III, VI].

J. Proudman, A. T. Doodson, \& G. Kennedy

1. R. So. London, Trans., v. 217A, 1917, p. 284-288. [IV].

G. N. RAMACHANDRAN

1. Indian Acad. Sci., Proc., v. 17A, 1943, p. 206-207. [I].

J. W. Strutt, baton Rayleigh (see also Bourget, P. A. Hansen, Stokes, Thomson)

1. London Math. So., Proc., v. 5, 1874, p. 121; note by A. CAYley, p. 124. [I].

12. Scientific Papers, v. 1, 1899, p. 192, 195.

2. London Math. So., Proc., v. 10, 1878, p. 7. [III].

2. Scientific Papers, Cambridge, Univ. Press, v. 1, p. 363-364.

3. Phil. Mag., s. 5, v. 8, 1879, p. 405. [V].

3. Scientific Papers, v. 1, p. 430.

4. Phil. Mag., s. 5, v. 34, 1892, p. 153. [III].

4. Scientific Papers, v. 3, 1902, p. 592.

5. Phil. Mag., s. 5, v. 34, 1892, p. 179. [III].

5. Scientific Papers, v. 3, p. 595-596.

6. R. So. London, Trans., v. 203A, 1894, p. 92-94. [IV].

6. Scientific Papers, v. 5, 1912, p. 155-156.

7. R. So. London, Proc., v. 84A, 1910, p. 39. [IV].

7. Scientific Papers, v. 5, 1912, p. 561.

8. Phil. Mag., s. 6, v. 30, 1915, p. 333, 337. [V]. J. C. P. MilLer drew our attention to this paper (with the following comment) which gives solutions [Airy integrals] of $s^{\prime \prime}=9 x s$, with pure imaginary argument $x=i \eta$, for $\eta=0(.1) 2.5$. Real and imaginary parts of two independent solutions are given, 3-5S.

Errata: For $\eta=.7$ all values are in error and should be $.9471,-.5122, .1796$, and .6868 respectively; also at $\eta=1.1$, for -2.007 , read -1.865 .

8. Scientific Papers, v. 6, 1920, p. 344.

9. Phil. Mag., s. 6, v. 36, 1918, p. 371. [II].

92. Scientific Papers, v. 6, 1920, p. 559.

F. REICHE, see LADENBURG 
E. R. REINSTEIN (see also Bourget)

1. Untersuchungen über die Transversalschwingungen der gleichförmig gespannten elliptisch oder kreisförmig begrenzten Vollmembran und Kreisringmembran sowie von Vollkreis- und Kreisringmembranen mit nach speziellen Gesetzen variierter ungleichförmiger Spannung (Diss. Göttingen), Leipzig, Barth, 1911. p. 75, 88. $15 \times 22.8 \mathrm{~cm}$. [I, II].

P. J. van RHIJN, see Kapteyn

C, RIBIÈRE

1. Paris, École Polyt., J., s. 2, cahier 6, 1901, p. 180-184. [III].

B. RiemanN \& H. M. Weber, see JAhNKe \& Emde $1_{1}$.

R. RODER

1. Institute of Radio Engineering, Proc., v. 10, 1931, p. 2175. [III].

L. M. Rodgers, see D. B. SMith

E. B. Rosa \& F. W. Grover

1. U. S. Bureau Standards, Bull., v. 8, 1912, p. 177, 226-228. [XI].

12. A. Russell, $A$ Treatise on the Theory of Alternating Currents, v. 1, Cambridge, University Press, second ed., 1914, an abridgement, p. 233.

H. A. Rowland

1. Phil. Mag., s. 5, v. 35, 1893, p. 414. [I].

G. RUDOLPH

1. Z. f. Physik, v. 111, 1938, graph, p. 532-533. [X].

R. RÜDENBURG

1. Z. angew. Math. Mech., v. 5, 1925, p. 366, graphs, p. 365, 370, 372-73, 388. [XI].

R. RUEDY

1. Canadian J. Research, v. 5A, 1931, p. 154, graph, p. 151. [I, III].

2. Canadian J. Research, v. 13A, 1935, p. 13. [I].

3. Canadian J. Research, v. 17A, 1939, graph, p. 12. [VI].

R. RÜHLE, see EMDE

A. Russell (see also Maclean, Rosa, Savidge)

1. Phys. So. London, Proc., v. 22, 1910, p. 447. [XI].

1. Phil. Mag., s. 6, v. 20, 1910, p. 605.

S. SAKURABa \& I. KimuRA

1. Kisho Shushi, Tokyo, Meteorological So. of Japan, J., s. 2, v. 11, no. 8, 1933, p. 372; (in Japanese). [XI]. The tables of $\operatorname{kei}^{\prime} x / \operatorname{ker}^{\prime} x$, and of bei' $x /$ ber $^{\prime} x$ are in $1_{1}$ only.

12. Geophysical Mag., Tokyo, v. 8, 1934, p. 140-141; (in English).

H. G. SAvidge

1. Phys. So. London, Proc., v. 22, 1910, p. 109-114. [XI].

12. Phil. Mag., s. 6, v. 19, 1910, p. 53-58.

13. A. Russell, $A$ Treatise on the Theory of Alternating Currents, v. 1, Cambridge, University Press, second ed., 1914. p. 230-232.

2. B.A.A.S., Report, 1915, p. 36-38. [XI].

2. DWIGHT $1_{1}, 1_{2}, 1_{3}$.

3. B.A.A.S., Report, 1916, p. 118-122. [XI].

S. A. SCHELKUNOFF

1. Electromagnetic Waves, New York, Van Nostrand, 1943, p. 323-324 and graphs, p. 265, 271, 327. [I, II, X, XI].

R. SChEUERMANN

1. Ueber die Gestalt und die Auflösung des fallenden Flüssigkeitsstrahles (Diss. Giessen), 1919, p. 252. [III].

12. Annalen d. Physik, s. 4, v. 60, 1919, p. 252.

M. A. SCHIRMANN

1. Akad. d. Wissen., Vienna, Sitzungsb., s. IIa, v. 127, 1918, p. 1628. [XI].

12. Annalen d. Physik, s. 4, v. 59, 1919, p. 537.

F. SCHLEICHER

1. Kreisplatten auf elastischer Unterlage . . ., Berlin, Springer, 1926, p. 136-147. [XI]. 
"Die Funktionswerte für $0<x<2,00$ sind neu berechnet. Die werte von $Z_{i}(x)$, für $2<x<6,0$ sind aus den Tafeln bei Jahnke und Emde [1 1 ] entnommen und die Werte von $d Z i / d x$ für 2,00 $<x<6,0$ aus denen von Jahnke und Emde berechnet worden," p. 135.

Errata: "Die neu berechneten Werte für $0<x<2,00$ sind teilweise bis auf zwei Einheiten der letzten Stelle unsicher, welch letztere deshalb durch kleine Ziffern gekennzeichnet wurde, . . ." SCHLEICHER, p. 135.

12. Hayashi 2, p. 111-118.

O. Schlömilch, see P. A. Hansen

H. SCHMICK

1. Z. tech. Physik, v. 6, 1925, p. 367-368. [I].

E. Schoenberg, see Nagaoka, Struve

J. F. SCHOUTEN

1. Physica, v. 7, 1940, p. 109. [I].

E. SCHRÖDINGER

1. Annalen d. Physik, s. 4, v. 44, 1914, graph, p. 923. [I].

O. T. Schultz, see Olson

F. A. Schulze (see also KIrchhoff)

1. Annalen d. Physik, s. 4, v. 24, 1907, p. 790. Of the 10 roots of the Kirchhoff equation ( $\sigma=1$ ) here given, the first $2(n=0)$ were given by RAYLEIGH, Theory of Sound, second ed., v. 1,1894, p. 367 . [III].

12. JAHNKE \& EMDE 11, (p. 160), $1_{2}$ (p. 283), $1_{3}-1_{5}$ (p. 234).

Errata: 8 errors of the table have been corrected in JAHNKE \& EMDE 1。 (p. 234).

T. E. W. SchumanN

1. Franklin Inst., J., v. 208, 1929, graphs, p. 412-413. [VIIi].

L. SCHWARZ

1. Luftfahrtforschung, v. 20, 1944, p. 352-372, graphs, p. 351. [X].

F. M. SCHWERD

1. Die Beugungserscheinungen aus den Fundamentalgesetzen der Undulationstheorie, analytisch entwickelt und in Bildern dargestellt, Mannheim, 1835, p. 70, [146]. [I].

12. Mascart, 1889 , p. 311-312.

13. Pernter, 1906, p. 441-442.

W. R. SEARS

1. Franklin Inst., J., v. 230,1940, p. 106-107. [X].

H. SeEliger

1. Bayer. Akad. d. Wissen, math.-natw. Abt., Abh., v. 19, 1897, p. 447-448. [X].

R. Seeliger, see OllendorfF.

W. SEITZ

1. Annalen d. Physik, s. 4, v. 16, 1905, p. 753-4, 758, 765. [I, II, XI]. Corrections, v. 19, 1906, p. 557-558.

2. Annalen d. Physik, s. 4, v. 21, 1906, p. 1020-1022, 1024-1027. [XI].

L. Silberstein

1. Phil. Mag., s. 6, v. 35,1918 , p. 45 ; graph p. 46. [IV, X]. This title was suggested by the Liverpool Index.

W. M. Smart (see also Eddington, Kapteyn)

1. Stellar Dynamics, Cambridge, University Press, 1938, p. 43, 127. [III, IV]. This title was suggested by the Liverpool Index.

B. A. Smith

1. Mess. Math., s. 2, v. 26, 1926, p. 98-101. [II]. The author states that error should be at most 2 units in the last place, but J. C. P. Miller in the Liverpool Index states that this limit is exceeded when $x \geq 8.5$.

12. JAHNKE \& EMDE $1_{1}$, p. 124-125.

2. Phil. Mag., s. 5, v. 45, 1898, p. 122, 123. [II]. The author states that there may be an error of 1 in the last place or possibly of 2 for $x>3$ or 4 . 
2. JAHNKE \& EMDE 11, p. 127-128. Between this table and the 4-place abridgement on p. 126 of a 21-place table of ALDis there are 35 cases of contradictory values.

3. A.S.C.E., Trans., v. 83,1921 , p. 2052-2055. [XI].

D. B. Smith, L. M. Rodgers \& E. H. TRaub

1. Franklin Institute, $J$., v. 237, 1944, p. 302-303. [I]. Zeros of $J_{n}(x)$ and $J_{n}{ }^{\prime}(x)$; these tables are believed to be "accurate to the extent that the last figure is within plus or minus two of the correct value" (p. 303).

G. A. V. SOWTER, see McLachlaN

F. STADIE

1. Annalen d. Physik, s. 4 , v. 86,1928 , p. 775 . [I].

G. STEí́

1. Am. J. Math., v. 38, 1916, p. 107. [X].

\section{J. STEINER}

11. Mat. és Termés. Értesitö, v. 12, 1893, p. 47, 49, 53-55. [I, X].

12. German transl. Math. naturw. Berichte a. Ungarn, v. 1, 1894, p. 366, 367, 372, 373. Errata: P. 54, for $x<25.5 ; J_{1}(20.1)=+0.082801, J_{1}(23)=-0.039519$; his second table should be used with caution. Liverpool Index.

H. STENZEL

1. Elek. Nach. Techn., v. 15, 1938, p. 73-75, graphs, p. 72. [IV].

2. Leitfaden zur Berechnung von Schallvorgängen, Berlin, Springer, 1939, p. 115-120. [X].

M. A. STERN

1. J.f. d. reine u. angew. Math., v. 22, 1841, p. 29, 34-35. [IV].

G. S. STEWARD

1. R. So. London, Trans., v. 225A, 1926, p. 149, 157, 171, and graph, p. 184. [I, V, X].

J. K. Stewart, see Colwell

G. G. STOKES

1. Cambr. Phil. So., Trans., v. 9, part 1, 1851, p. 181, 184, 186. [I, V].

12. Mathematical and Physical Papers, v. 2, Cambridge, Univ. Press, 1883, p. 349, 353, 355. Of the tables of zeros of $A(m)$ and $A^{\prime}(m)$, p. 181 (349), MASCART reproduces the following zeros of $A(m): s=1(1) 10(2) 14(3) 20(5) 50$, and all 10 zeros of $A^{\prime}(m)$; PeRnTER, p. 517, reproduces the first 10 zeros of both $A(m)$ and $A^{\prime}(m)$ and adds, to 3D, the first 10 maxima of $A^{2}(m)$. Stokes's table of $x_{s} / \pi$, and $x_{s}^{\prime} / \pi$, p. $186(355)$, is reproduced in RAYLEIGH, Theory of Sound, London, v. 1, 1877, p. 274 (German ed., Brunswick, 1879, p. 363); second ed., 1894, and reprints of 1926, 1929, 1940, p. 330. This table is also in Byerly 1, p. 286; in Dale, p. 83; and in W. LÁsKa, Sammlung von Formeln der reinen und angewandten Mathematik, zweite Lieferung, Brunswick, 1889 , p. 390.

M. J. O. STRUTt

1. Annalen d. Physik, s. 4, v. 82, 1927, graphs p. 610, 615. [XI].

2. Archiv f. Elektrotechnik, v. 19, 1928, p. 429. [XI].

H. STRUVE

1. Annalen d. Physik, s. 2, v. 15,1882 , p. 80 . [XII]. This paper is an extract of a dissertation, Univ. of Dorpat, 1881.

2. Akad. Nauk, Leningrad, Mémoires, s. 7, v. 30, no. 8, 1882, p. 35-36, 54-55. [X].

2. Annalen der Physik, s. 2, v. 17, 1882, p. 1016; one of the tables in 2 .

2. Partially reprinted table of $2_{2}$ in GraY \& Mathews $1_{1}$ (p. 207), and $1_{2}$ (p. 218).

2. Handbuch der Astrophysik, v. 21, Grundlagen der Astrophysik, part 2, Berlin, 1929; "Theoretische photometrie" by E. SchoenBERG, p. 117, 118.

\section{P. SzYMANSKI}

1. Journ. de Math., s. 9, v. 11, 1932, p. 90. [XII].

M. TAKAGI (see also LoDGE)

i. Tôhoku Imp. Univ., Science Reports, s. 1 (Mathematics, Physics, Chemistry), v. 28, 1939, p. 76-84. [III]. See $M T A C$, p. 321. 
S. TAKAYA

1. Imperial Marine Observatory, Kobe, Japan, Memoirs, v. 4, no. 1, 1930, p. 8-9, $11-12,14 .[\mathrm{XI}]$.

K. Terazawa

1. R. So. London, Proc., v. 92A, 1916, p. 77-80. [XII].

G. S. TERRY

1. Duodecimal Arithmetic, London, New York, Toronto, Longmans, Green, 1938, G. THILO p. $396-399.23 .8 \times 29.2 \mathrm{~cm}$. [I, IV].

1. Annalen d. Physik, s. 4, v. 62, 1920, p. 552-568. [I, II].

A. J. Thompson, see BAASMTC 4

J. J. Thomson (see also Bourget)

1. Notes on Recent Researches in Electricity and Magnetism, Oxford, 1893, p. 276, 347, 353,357 . [I, XI]. The table on p. 276 is reproduced in GRAY \& MATHEws $1_{1}$, p. 155; 12, p. 170; that on p. 347 from RaYLEIGH, Theory of Sound, v. 2, London, 1878,

B. THÜRING p. 266; that on p. 357 from LAMB 3.

1. Astr. Nach., v. 235, 1929, cols. 49-51. [I].

H. W. TOBEY, see Hawkins

T. TOBIISI, see OKAYA

F. TÖLKE

1. Besselsche und Hankelsche Zylinderfunktionen nullter bis dritter Ordnung vom Argument $r \sqrt{i}$, Stuttgart, Wittwer, 1936, 92 p. $17.7 \times 25.1 \mathrm{~cm}$. [XI].

Tables: ber $x\left(J_{01}\right)$, bei $x\left(J_{02}\right)$, ber $^{\prime} x$, bei' $x$, ber $_{2} x$, bei $x,-\left(\operatorname{ber}_{3} x \pm\right.$ bei $\left._{3} x\right) / 2$, and 8 corresponding forms for the ker and kei functions.

Errata: The following report on some errors in $J_{01}$ and $J_{02}$ was prepared by H. E. SALZER and E. ISAACSON: The values of these functions are identical with the real and imaginary parts of $J_{0}(z)$ along the $45^{\circ}$ ray. TOELKE's values, which are tabulated up to $r=21$ to $4 \mathrm{~S}$ (in a few places, to 3S), were proofread as far as $r=10$ against the NYMTP 10-decimal manuscript of $J_{0}(z)$. The last significant figure was found to be incorrect by a unit (this includes doubtful roundings) for 55 values of $J_{01}$, and 76 values of $J_{02}$. In addition, the following errors of more than a unit in the last figure were found for $J_{01}$ and $J_{02}$.

$\begin{array}{cccccc}r & \begin{array}{c}J_{01} \\ \text { for }\end{array} & \text { read } & r & \begin{array}{c}J_{02} \\ \text { for }\end{array} & \text { read } \\ 7.21 & -0.5029 & -0.5034 & 6.22 & 9.888 & 9.890 \\ 7.22 & -0.3303 & -0.3309 & 7.56 & 29.97 & 29.99 \\ 7.23 & -0.1556 & -0.1562 & 8.65 & 33.56 & 33.54 \\ 7.24 & 0.0213 & 0.0208 & 9.32 & 9.197 & 9.195 \\ 7.25 & 0.2006 & 0.2001 & 9.36 & 6.657 & 6.655 \\ 7.26 & 0.3821 & 0.3816 & 9.41 & 3.276 & 3.274 \\ 7.27 & 0.5661 & 0.5655 & 9.42 & 2.572 & 2.570 \\ 7.28 & 0.7520 & 0.7516 & 9.45 & 0.4014 & 0.4003 \\ 7.29 & 0.9404 & 0.9400 & 9.46 & -0.3414 & -0.3423 \\ 7.37 & 2.530 & 2.533 & 9.48 & -1.855 & -1.857 \\ & & & 9.54 & -6.636 & -6.638 \\ & & & 9.55 & -7.469 & -7.471 \\ & & & 9.62 & -13.57 & -13.59\end{array}$

J. Topping \& A. E. LudlaM

1. R. So. London, Trans., v. 226A, 1927, p. 135-155. [III].

M. J. DiToro, see JAHNKE \& EMde 1 .

E. H. Traub, see D. B. SMith

R. TRUELL

1. J. Applied Physics, v. 14, 1943, graphs p. 351-352. [II]. 


\section{F. J. TURTON}

1. Phil. Mag., s. 7, v. 32, 1941 , p. $465-466$. [I].

A. TVERITIN

1. Ms. table of $J_{2}(x)$ for $x=[.01(.01) 10 ; 5 \mathrm{D}]$ at Dnepropetrovsk (Ekaterinoslav). A copy was at the Institute for Applied Mathematics, University of Berlin. Listed in R. v. Mises, Verzeichnis berechneter Funktionentafeln, part 1, Berlin, 1928, p. 11. [I, II].

G. E. UhLENBECK, see NoRDSIECK

A. WAGNER

1. Beiträge zur Geophysik, v. 24, 1930, p. 395, 399-400, 409. [III, X].

A. WALther, see JAHNKE \& EMde

G. WANNIER

1. Annalen d. Physik, s. 5 , v. 24,1935 , p. 575,579 , graph, p. 577 . [IV, XII].

P. F. WARD

1. Phil. Mag., s. 6, v. 25, 1913, p. 90. [I, III].

G. N. Watson (see also Macdonald)

1. R. So. London, Proc., v. 94A, 1918, p. 193, 204-205. [I, V].

2. Phil. Mag., s. 6, v. 35, 1918, p. 369. [X].

3. A Treatise on the Theory of Bessel Functions, Cambridge, University Press, 1922. Tabulation of Bessel Functions, p. 654-752. [I-V, IX, X]. Amer. ed., N. Y., 1944. Tables: T. I: $J_{0}(x), Y_{0}(x),\left|H_{0}^{(1)}(x)\right|, \arg H_{0}^{(1)}(x), H_{0}(x), J_{1}(x), Y_{1}(x),\left|H_{1}^{(1)}(x)\right|$, $\arg H_{1}^{(1)}(x), H_{1}(x)$; T. II : $e^{-x} I_{0}(x), e^{-x} I_{1}(x), e^{x} K_{0}(x), e^{x} K_{1}(x), e^{x} ;$ T. III : $J_{1 / 3}(x)$, $Y_{1 / 3}(x),\left|H_{1 / 3}^{(1)}(x)\right|, \arg H_{1 / 3}^{(1)}(x), e^{x} K_{1 / 3}(x) ;$ T. IV: $J_{n}(x), n=1-20$ (from LoMmeL 2 with corrections); $Y_{n}(x), n=1-13 ; e^{-x} I_{n}(x), n=2-5 ; K_{n}(x), n=0-10 ; \mathrm{T}$. V: $J_{ \pm(n+\xi)}(x) ; \frac{1}{2} \int_{0}^{x} J_{ \pm\}}(t) d t$ (almost wholly from LoMmeL 3 , with corrections); T. VI : $J_{n}(n), \quad n^{1 / 8} J_{n}(n), \quad J_{n}{ }^{\prime}(n), \quad n^{2 / 8} J_{n}{ }^{\prime}(n), \quad-Y_{n}(n), \quad-n^{1 / 8} Y_{n}(n), \quad Y_{n}{ }^{\prime}(n), \quad n^{2 / 8} Y_{n}{ }^{\prime}(n) ;$ T. VII: Zeros of $J_{n}(x)\left(x_{s}, x_{0}^{\prime}\right.$, from WILLSON and PEIRCE), $Y_{n}(x), n=0-5, \pm 1 / 3$; and of $J_{-1 / 8}(x) \pm J_{1 / 8}(x) ;$ T. VIII: $\frac{1}{2} \int_{0}^{x} J_{0}(t) d t, \frac{1}{2} \int_{0}^{x} Y_{0}(t) d t$.

Errata: In T. I, $Y_{1}(2.96)$, for .3136280, read .3136281; and in T. II, at $x=.14$, there are two errors, namely: in $e^{x} K_{0}(x)$ for 2.4122685 , read 2.4123173; in $e^{x} K_{1}(x)$, for 8.0076828 , read 8.0076794 (BAASMTC 1 , p. xvii). For various errors in T. V, see LOMMEL 3. Some errors are corrected in the Amer. ed.

4. R. So. London, Proc., v. 130A, 1930, p. 37. [X].

5. Accad. d. Lincei, Rendiconti, cl. d. Sci., s. 6, v. 27, 1938, p. 525-528. [II].

H. A. WebB \& J. R. Airey

1. Phil. Mag., s. 6, v. 36, 1918, p. 138-141. [III].

H. M. WEBER, see RIEMANN

A. G. Webster

1. B.A.A.S., Report, 1912, p. 57-68. [XI].

12. Dwight $1_{1}, 1_{2}, 1_{3}$.

Errata: corrections of 36 entries for bei' $x$ are given (to 6D instead of 9D) by SAVIDGE 3, p. 122. Webster's bei' 3.7 should be 0.131486760 and bei 8.9 should be

D. L. WEBSTER -28.002867538 (Dwight 4, p. 787).

1. J. Appl. Physics, v. 10, 1939, graph, p. 504. [I].

A. M. WEINBERG

1. Bull. Math. Biophysics, v. 3, 1941, p. 39-45. [II].

W. WEINREICH

1. Z. Math. Physik, v. 63, p. 46-47, 55-56, graph p. 57. 1914. [XI]. Errata: See MACLEAN.

W. WESSEL

1. Hochfrequenztechnik u. Elektroakustik, v. 54, 1939, p. 68. [XII].

A. Westgren

1. Arkiv f. Matem., Astr. o. Fysik, v. 13, no. 14, 1918, p. 4-5. [III]. 
F. WEVER, see W. FISCHER

J. A. WHEELER, see YosT

T. T. Whitehead, see ChapMaN

R. W. Willson \& B. O. Peirce

1. Am. Math. So., Bull., v. 3, 1897, p. 153-155. [1]. $x_{s} ; 10 \mathrm{D}, \log x_{s} ; 10 \mathrm{D}, J_{1}\left(x_{s}\right) ; 8 \mathrm{D}$, $\log \left|J_{1}\left(x_{s}\right)\right| ; 7 \mathrm{D}$.

12. Gray \& Mathews $1_{2}$, T. III, p. 300, $x_{s}, J_{1}\left(x_{s}\right), s=11-40$.

1. AIREY 17 , p. 241, reprint, to 7D, of $x_{s}, x_{s^{\prime}}^{\prime}, J_{1}\left(x_{s}\right), J_{0}\left(x_{s}^{\prime}\right), s=1(1) 40$.

14. Watson 3, T. VII, p. 748, $x$, to 7D.

Errata: In each of the printings $\left(1_{1}-1_{3}\right)$ there is an error in $J_{1}\left(j_{0,25}\right)$, for +.07635913 ,

J. R. WILTON read +.07636 383. (Davis \& KirkhaM, p. 760).

1. Mess. Math., s. 2, v. 56, 1927, p. 179. [X].

A. W. WitkowskI

1. Tablice Matematyczno-Fiszyczne, Warsaw, 1904, p. 84-89. [I]. This title was suggested by the Liverpool Index. The copy inspected belonged to the Amer. Math. So.; a film copy is at Brown University.

J. W. Wrench Jr., see Bessel, Colwell \& Hardy, Maclean

D. A. WRIGHT

1. Phil. Mag., s. 7, v. 24, 1937, graphs, p. 11. [XI].

D. WRINCH

1. R. So. London, Proc., v. 101A, 1922, p. 501, 505. [I, III]. Compare AIREy 2.

H. E. H. WRINCH \& D. M. WRINCH

1. Phil. Mag., s. 6, v. 45,1923 , p. 847,848 . [III, IV].

2. Phil. Mag., s. 6, v. 47,1924 , p. 64 , graphs, p. 65 . [III, IV].

F. L. Yost, J. A. WheEler \& G. BREIT

1. Terrestrial Magnetism, v. 40, 1935, p. 446, plate 11. [IV]. This title was suggested by the Liverpool Index.

2. Physical Review, s. 2, v. 49, 1936, p. 185. [III].

H. ZANSTRA

1. Physica, v. 2, 1935, graphs, p. 818-819. [III].

J. ZENNECK (see also MACLEAN)

1. Annalen d. Physik, s. 4, v. 11, 1903, p. 1138-1141. [XI].

F. ZERNIKE

1. Physica, v. 1, 1934, graph p. 703. [I].

\section{ADDENDA}

P. 226, IIA 2 , 17A. 5D; 4D, $\log \left[e^{-\rightarrow} I_{0}(x)\right]$, WAGNER, $x=0(.1) 2(.2) 4(.5) 7(1) 20 ; 25(5) 65$.

P. 246, add: $\operatorname{IXA}_{1}, 15.5 \mathrm{D}, \log \left[(2 / x)\left\{2 \pi^{-1}-H_{1}(i m x)-I_{1}(m x)\right\}\right]$, Michell $2, m=3(2) 11$, $x / \pi=[1(.1) 2 ; 5 \mathrm{D}]$.

P. 253: The Michell functions $m_{1}$ and $m_{2}$ are here expressed in terms of the ber and bei

functions. The following results may be added $\left(z=2 x^{\mathrm{b}}\right)$ :

$$
\begin{aligned}
& x^{3} m_{\mathrm{z}}=(2-2 \gamma) \text { bei' }^{\prime}(z)-\frac{1}{2} \pi \text { ber' }^{\prime}(z)-2 \text { kei' }^{\prime}(z), \\
& x^{3} m_{4}=-2 \gamma \text { ber }^{\prime}(z)+\frac{1}{2} \pi \text { bei }^{\prime}(z)-2 \text { ker' }^{\prime}(z) .
\end{aligned}
$$

\section{CORRIGENDA}

P. $137,1.4$, for $P_{4}\left(\cos 7^{\circ}\right)$, read $P_{4}\left(\cos 70^{\circ}\right)$.

P. 178, 1. 22, for $r=e^{i \theta}$, read $r e^{i \theta}$.

P. $180,1.13$, read $\tan ^{-1} x, x=[0.000(0.001) 1.000 ; 4 \mathrm{D}] ; 1.20$, for $1-6 \mathrm{~S}$, read $7 \mathrm{~S}$, and for $5-7 \mathrm{D}$, read 7D; 1.21 , for $2-7 \mathrm{D}$, read 7D; 1.32 , for $(0-1)$, read $(0.1)$.

P. 191, 1. 5, for Macrobert's, read MacRobert's.

P. 194, MTE 29, last 1., for $I_{2 x}^{n}$ (HANSEN), read $I_{i x}^{n}$ (HANSEN). 